\title{
Smooth $p$-adic analytic spaces are locally contractible
}

\author{
Vladimir G. Berkovich ${ }^{\star}$ \\ Department of Theoretical Mathematics, The Weizmann Institute of Science, P.O.B. 26, \\ 76100 Rehovot, Israel
}

Oblatum 18-III-1998 \& 26-X-1998 / Published online: 10 May 1999

\section{Contents}

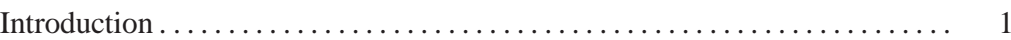

1. Pluri-nodal and poly-stable morphisms and poly-stable fibrations of

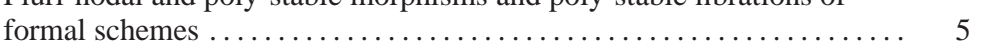

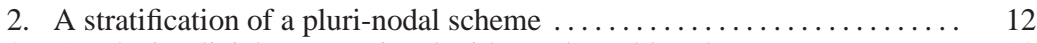

3. A polysimplicial set associated with a poly-stable scheme .......... 18

4. A colored polysimplicial set associated with a poly-stable formal scheme 31

5. The homotopy type of the generic fibre of a poly-stable formal scheme. . 37

6. A polysimplicial set associated with a poly-stable fibration of schemes.. 52

7. A lifting of a homotopy for a poly-stable morphism of formal schemes .. 59

8. The homotopy type of the generic fibre of a poly-stable fibration of

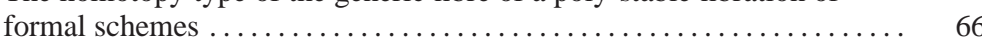

9. Local contractibility of smooth analytic spaces .............. 74

10. Finiteness and stability of cohomology of certain analytic spaces ...... 81

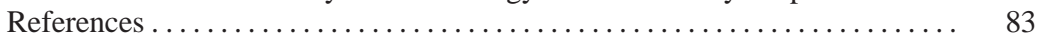

\section{Introduction}

Recall that a topological space $X$ is called contractible if there exist a continuous mapping $\Phi: X \times[0,1] \rightarrow X$ and a point $x_{0} \in X$ such that $\Phi(x, 0)=x$, $\Phi(x, 1)=x_{0}$ and $\Phi\left(x_{0}, t\right)=x_{0}$ for all $x \in X$ and $0 \leq t \leq 1$. A topological space is called locally contractible if each point of it has a fundamental system of contractible open neighborhoods. A trivial example of such a space is a topological manifold. A non-trivial example is a complex analytic space. Local contractibility implies important properties of a topological space. For example, such a space has a universal covering and, if it is connected, the universal covering is a Galois one with the Galois group isomorphic to the fundamental group of the space [Spa, Ch. 2]. Furthermore, if such a space

* The research was supported by Minerva Foundation, Germany, and US-Israel Binational Science Foundation. Parts of the work were done during author's stay at Centre Emile Borel - UMS 839 IHP (CNRS/UPMC) - Paris, and at IHES, Bures-sur-Yvette. 
is paracompact, its cohomology groups with coefficients in a constant sheaf coincide with the singular cohomology groups [Spa, Ch. 6]. The statement on local contractibility of smooth $p$-adic analytic spaces formulated in the title is one of the main results of this paper. What is the meaning of the statement?

First of all, the statement does not make sense for rigid analytic spaces, introduced by J. Tate in [Tate] as $p$-adic analogs of complex analytic spaces, since the topology on rigid spaces is totally disconnected. The statement is about the analytic spaces introduced by the author in [Ber1] and [Ber2]. They are closely related to rigid spaces. Namely, the category of analytic spaces has a full subcategory of so called Hausdorff strictly analytic spaces which admits a fully faithful functor to the category of quasiseparated rigid spaces, and this functor induces an equivalence between the category of paracompact strictly analytic spaces and that of quasiseparated rigid spaces that have an admissible affinoid covering of finite type. (Actually both equivalent categories include all of the spaces needed in practice.) Moreover, the above functor preserves the cohomology groups of abelian sheaves and the categories of coherent sheaves. But the advantage of the new spaces is in their nice topology. For example, each point has a fundamental system of open neighborhoods which are locally compact, countable at infinity and arcwise connected. The topological dimension of a paracompact analytic space is at most its (algebraic) dimension and is equal to it if the space is strictly analytic. Furthermore, a scheme $\mathcal{X}$ of locally finite type is separated (resp. proper, resp. connected) if and only if the associated analytic space $\mathcal{X}^{\mathrm{an}}$ is Hausdorff (resp. compact, resp. arcwise connected), and the topological dimension of $\mathcal{X}^{\text {an }}$ is equal to the dimension of $\mathcal{X}$. Such spaces as the projective space $\mathbf{P}^{d}$, the affine space $\mathbf{A}^{d}$, open and closed polydiscs in it and the Drinfeld upper half-space $\Omega^{d}$ [Dr1] are contractible, and the Tate elliptic curve is homotopy equivalent to the circle. In [Ber1] we also proved that analytic curves are locally contractible. The nice topology of the analytic spaces was essentially used in their various applications ([Ber1]-[Ber7], [Che], [deJ1]). Of course, local contractibility of the analytic spaces proven in this paper for smooth spaces is one of the most desirable properties of them. Besides the consequences mentioned at the beginning of the introduction, it provides an evidence of the existence of integration on $p$-adic analytic spaces. What is the difficulty in proving this property?

Recall that a smooth complex analytic space is locally isomorphic to an open polydisc and, therefore, it is contractible for trivial reasons. Although contractibility of $p$-adic open polydiscs was established in [Ber1], the difficulty of the $p$-adic case is in the fact that a smooth $p$-adic analytic space is not in general locally isomorphic to an open polydisc. The simplest example is an open annulus in the affine line. (In the language of rigid analytic geometry, this means that the open annulus has no an admissible covering by open or closed discs.) In the proof of local contractibility of analytic curves, a key ingredient was the semi-stable reduction theorem for curves. It was used to show that a smooth curve minus a certain discrete 
subset of points is a disjoint union of open discs and annuli. Of course, after A.J. de Jong proved his remarkable results in [deJ2] and [deJ3], the author started thinking of applying them to the problem of local contractibility of $p$-adic analytic spaces. What is done in this paper is a higher dimensional generalization of the one-dimensional case from [Ber1, Ch. 4], obtained with the use of constructions from [Ber1], [Ber3] and [Ber5] together with the results of de Jong. But even for curves, the picture obtained is more precise. As for local contractibility of the analytic spaces with singularities, one probably needs new ideas. We now give a brief summary of the material which follows.

Let $k$ be a field complete with respect to a non-Archimedean valuation (which is not assumed to be nontrivial). The main body of the paper (§§1-8) is devoted to a description of the homotopy type of the generic fibre $\mathfrak{X}_{\eta}$ of a formal scheme $\mathfrak{X}$ locally finitely presented over the ring of integers $k^{\circ}$ in terms of a combinatorial object associated with the closed fibre $\mathfrak{X}_{s}$. Notice that in general the closed fibre $\mathfrak{X}_{s}$ does not determine the homotopy type of the generic fibre $\mathfrak{X}_{\eta}$. But, it turns out, that $\mathfrak{X}_{s}$ does determine the homotopy type of $\mathfrak{X}_{\eta}$ for a class of formal schemes, called poly-stable fibrations and related to $G$-pluri nodal fibrations from [deJ3]. In $\S 1$ we introduce this class. It consists of the formal schemes $\mathfrak{X}$ provided with a decomposition of the canonical morphism to $\operatorname{Spf}\left(k^{\circ}\right)$ in the form $\mathfrak{X}=\mathfrak{X}_{l} \stackrel{f_{l-1}}{\rightarrow} \mathfrak{X}_{l-1} \stackrel{f_{l-2}}{\rightarrow} \ldots \stackrel{f_{0}}{\rightarrow} \mathfrak{X}_{0}=\operatorname{Spf}\left(k^{\circ}\right)$, where $f_{i}$ are poly-stable morphisms. The latter are natural generalizations of fibre products of semistable morphisms. For technical reasons we also introduce a broader class of pluri-nodal morphisms. In $\S 2$ we provide a pluri-nodal scheme $\mathcal{X}$ over a field with a stratification. The latter defines a simplicial set $N(\mathcal{X})$ which is, more or less, the nerve of the partially ordered set of the strata.

In $\S 3$ we introduce a category of polysimplicial sets. It is a symmetric strict monoidal category which is better adapted to a combinatorial description of poly-stable fibrations than the category of simplicial sets. We associate with a poly-stable scheme $\mathcal{X}$ over a field a polysimplicial set $\mathbf{C}(\mathcal{X})$. We also construct functors from the category of polysimplicial sets to those of partially ordered sets and of simplicial sets. The former takes $\mathbf{C}(\mathcal{X})$ to the partially ordered set of strata of $\mathcal{X}$, and the latter preserves geometric realizations and takes $\mathbf{C}(\mathcal{X})$ to $N(\mathcal{X})$. In $\S 4$ we provide the polysimplicial set $\mathbf{C}\left(\mathfrak{X}_{s}\right)$ associated with the closed fibre of a poly-stable formal scheme $\mathfrak{X}$ over $k^{\circ}$ with an extra structure. For this we introduce a category of colored polysimplicial sets and a geometric realization functor from it to the category of topological spaces endowed with a sheaf of monoids of continuous functions with values in the unit interval $[0,1]$. (The colored polysimplicial sets are precisely the combinatorial objects that give rise to the the BruhatTits buildings of semi-simple algebraic groups from [BrTi].) We associate with $\mathfrak{X}$ a colored polysimplicial set $\mathbf{L}(\mathfrak{X})$. The underlying topological space of $|\mathbf{L}(\mathfrak{X})|$ is homeomorphic to $\left|\mathbf{C}\left(\mathfrak{X}_{s}\right)\right|$. In $\S 5$ we construct a proper deformation retraction of $\mathfrak{X}_{\eta}$ to a closed subset $S(\mathfrak{X})$, called the skeleton of $\mathfrak{X}$. 
This retraction possesses nice properties. In particular, it commutes with all automorphisms of $\mathfrak{X}$ and induces a deformation retraction of each Zariski open subset of $\mathfrak{X}_{\eta}$ to its intersection with $S(\mathfrak{X})$. Furthermore, we provide the closed fibre of $\mathfrak{X}$ with an étale sheaf of monoids which is closely related to the logarithmic structures of Fontaine-Illusie (see Remark 5.12(ii)). It is used to endow the skeleton $S(\mathfrak{X})$ with a sheaf of monoids of continuous functions with values in $[0,1]$. Finally, we construct a functorial isomorphism $|\mathbf{L}(\mathfrak{X})| \stackrel{\sim}{\rightarrow} S(\mathfrak{X})$. The simplest consequence of the results of $\S 5$ tells that the analytification of a scheme, isomorphic to an open subscheme of a proper scheme with good reduction, is contractible.

We want to emphasize that there is a strong similarity between the results of $\$ 5$ and the Fontaine-Jannsen conjecture (already proven) which relates $p$-adic étale cohomology of the generic fibre $\mathcal{X}_{\eta}$ of a proper semi-stable scheme $\mathcal{X}$ over $k^{\circ}$, where $k$ is a finite extension of $\mathbf{Q}_{p}$, and crystalline cohomology of the closed fibre $\mathcal{X}_{s}$ endowed with the logarithmic structure induced from $\mathcal{X}$ (see [Tsu]).

In $\S \S 6-8$ we extend the results of $\S 5$ to poly-stable fibrations. Namely, in $\S 6$ we associate with a poly-stable fibration $\underline{\underline{X}}=\left(\mathcal{X}_{l} \rightarrow X_{l-1} \rightarrow \cdots \rightarrow\right.$ $\left.\mathcal{X}_{0}=\operatorname{Spec}(K)\right)$ over a field $K$ a polysimplicial set $\mathbf{C}(\underline{\mathcal{X}})$ so that the partially ordered set associated with $\mathbf{C}(\underline{\mathcal{X}})$ is isomorphic to the set of strata of $\mathcal{X}_{l}$. In $\S 7$ we construct for a poly-stable morphism of formal schemes $\mathfrak{Y} \rightarrow \mathfrak{X}$ a lifting of a homotopy of certain type from $\mathfrak{X}_{\eta}$ to $\mathfrak{Y}_{\eta}$. In $\S 8$ we construct for a poly-stable fibration $\underline{\mathfrak{X}}=\left(\mathfrak{X}_{l} \rightarrow \mathfrak{X}_{l-1} \rightarrow \cdots \rightarrow \mathfrak{X}_{0}=\operatorname{Spf}\left(k^{\circ}\right)\right)$ of formal schemes over $k^{\circ}$ a strong deformation retraction of $\mathfrak{X}_{l, \eta}$ to a closed subset $S(\underline{\mathfrak{X}})$, called the skeleton of $\underline{\mathfrak{X}}$, and a functorial homeomorphism $\left|\mathbf{C}\left(\underline{\mathfrak{X}}_{s}\right)\right| \stackrel{\sim}{\rightarrow} S(\underline{\mathfrak{X}})$. The latter possesses the same nice properties as the retraction for poly-stable formal schemes. The results of $\$ 8$ suggest that a generalization of the Fontaine-Jannsen conjecture for compositions of proper semi-stable morphisms might exist.

In $\S 9$ the valuation on the ground field $k$ is assumed to be nontrivial. (In all other sections this is not assumed.) First of all, the notion of smoothness we work with is that introduced in [Ber2, §3]. It corresponds better to the complex analytic smoothness but is more restrictive than the rigid analytic one. Namely, a strictly analytic space is smooth if and only if it is rigid analytically smooth and has no boundary (in the sense of [Ber2, §1.5]). We prove local contractibility of the analytic spaces locally embeddable in a smooth space. This class is broader than that of smooth spaces. It contains all strictly analytic domains in smooth spaces and all rigid analytically smooth affinoid spaces. As we mentioned at the beginning of the introduction, the result implies that any analytic space locally embeddable in a smooth space has a universal covering. Finally, in $\S 10$, we use the same methods to prove the following result. Let $\mathcal{X}$ be a separated scheme of finite type over $k$ and $X=X^{\mathrm{an}}$. Then the abelian groups $H^{q}(|X|, \mathbf{Z})$ and $H_{c}^{q}(|X|, \mathbf{Z})$ are finitely generated, and there exists a finite separable extension $k^{\prime}$ of $k$ such that for any non- 
Archimedean field $K$ over $k^{\prime}$ one has $H^{q}\left(\left|X \widehat{\otimes} k^{\prime}\right|, \mathbf{Z}\right) \stackrel{\sim}{\rightarrow} H^{q}(|X \widehat{\otimes} K|, \mathbf{Z})$ and $H_{c}^{q}\left(\left|X \widehat{\otimes} k^{\prime}\right|, \mathbf{Z}\right) \stackrel{\sim}{\rightarrow} H_{c}^{q}(|X \widehat{\otimes} K|, \mathbf{Z})$. What is proven in $\S 10$ and the results from $\S 8$ give strong evidence for the following conjecture. Let $X$ be a compact analytic space and $Y$ a Zariski closed subset of $X$. Then (a) the pair $(|X|,|Y|)$ is homotopy equivalent to a compact polyhedral pair $(A, B)$ with $\operatorname{dim}(A) \leq \operatorname{dim}(X)$ and $\operatorname{dim}(B) \leq \operatorname{dim}(Y)$, and (b) there exists a finite separable extension $k^{\prime}$ of $k$ such that for any non-Archimedean field $K$ over $k^{\prime}$ the canonical map of pairs $(|X \widehat{\otimes} K|,|Y \widehat{\otimes} K|) \rightarrow\left(\left|X \widehat{\otimes} k^{\prime}\right|,\left|Y \widehat{\otimes} k^{\prime}\right|\right)$ is a homotopy equivalence.

As is clear from the above, this work is very much stimulated by the work of A.J. de Jong, and I am very grateful to him for the help in understanding his results. I am very grateful to V. Hinich for many useful discussions and, especially, for his help in my search for appropriate simplicial tools.

\section{Pluri-nodal and poly-stable morphisms and poly-stable fibrations of formal schemes}

In this section we introduce several classes of morphisms of formal schemes which will be objects of study in this paper. The definitions are given for arbitrary formal schemes in order to apply them to usual schemes (which are a particular case of formal schemes).

First of all, recall that a covering of a topological space $X$ by subsets $\left\{X_{i}\right\}_{i \in I}$ is called locally finite if each point of $X$ has an open neighborhood which has a non-empty intersection with at most finite number of $X_{i}$ 's. If all of the subsets are open, this is equivalent to the fact that each point of $X$ is contained in at most finite number of $X_{i}$ 's.

Let $\varphi: \mathfrak{Y} \rightarrow \mathfrak{X}$ be a morphism of formal schemes. It is said to be locally finitely presented if the preimage of every open affine subscheme $\operatorname{Spf}(A)$ is a locally finite union of open affine subschemes of the form $\operatorname{Spf}\left(A\left\{T_{0}, \ldots, T_{n}\right\} /\left(f_{1}, \ldots, f_{m}\right)\right)$. All of the morphisms we consider will be assumed to be locally finitely presented. Furthermore, $\varphi$ is said to be étale if, for each ideal of definition $\mathscr{g} \subset \mathcal{O}_{\mathfrak{X}}$, the induced morphism of schemes $\left(\mathfrak{Y}, \mathcal{O}_{\mathfrak{Y}} / \mathcal{G O}_{\mathfrak{Y}}\right) \rightarrow\left(\mathfrak{X}, \mathcal{O}_{\mathfrak{X}} / \mathscr{g}\right)$ is étale. Finally, $\varphi$ is said to be smooth if, for every point $y \in \mathfrak{Y}$, there exist an open affine neighborhood $\mathfrak{X}^{\prime}=\operatorname{Spf}(A)$ of $\varphi(y)$ and an open neighborhood $\mathfrak{Y}^{\prime} \subset \varphi^{-1}\left(\mathfrak{X}^{\prime}\right)$ of $y$ such that the induced morphism $\mathfrak{Y}^{\prime} \rightarrow \mathfrak{X}^{\prime}$ goes through an étale morphism $\mathfrak{Y}^{\prime} \rightarrow \operatorname{Spf}\left(A\left\{T_{0}, \ldots, T_{n}\right\}\right)$.

Definition 1.1. Let $\varphi: \mathfrak{Y} \rightarrow \mathfrak{X}$ be a locally finitely presented morphism of formal schemes.

(i) $\varphi$ is said to be strictly pluri-nodal if locally in the Zariski topology it is a composition of étale morphisms and morphisms of the form $\operatorname{Spf}(A\{u, v\} /(u v-a)) \rightarrow \operatorname{Spf}(A), a \in A$.

(ii) $\varphi$ is said to be pluri-nodal if there exists a surjective étale morphism $\mathfrak{Y}^{\prime} \rightarrow \mathfrak{Y}$ such that the induced morphism $\mathfrak{Y}^{\prime} \rightarrow \mathfrak{X}$ is strictly pluri-nodal. 
The following definition introduces an important class of pluri-nodal morphisms.

Definition 1.2. Let $\varphi: \mathfrak{Y} \rightarrow \mathfrak{X}$ be a locally finitely presented morphism of formal schemes.

(i) $\varphi$ is said to be strictly poly-stable if, for every point $y \in \mathfrak{Y}$, there exist an open affine neighborhood $\mathfrak{X}^{\prime}=\operatorname{Spf}(A)$ of $\varphi(y)$ and an open neighborhood $\mathfrak{Y}^{\prime} \subset \varphi^{-1}\left(\mathfrak{X}^{\prime}\right)$ of $x$ such that the induced morphism $\mathfrak{Y}^{\prime} \rightarrow \mathfrak{X}^{\prime}$ goes through an étale morphism $\mathfrak{Y}^{\prime} \rightarrow \operatorname{Spf}\left(B_{0}\right) \times \mathfrak{X}^{\prime} \cdots \times{ }_{\mathfrak{X}^{\prime}} \operatorname{Spf}\left(B_{p}\right)$, where each $B_{i}$ is of the form $A\left\{T_{0}, \ldots, T_{n}\right\} /\left(T_{0} \cdot \ldots \cdot T_{n}-a\right)$ with $a \in A$ and $n \geq 0$.

(ii) $\varphi: \mathfrak{Y} \rightarrow \mathfrak{X}$ is said to be poly-stable if there exists a surjective étale morphism $\mathfrak{Y}^{\prime} \rightarrow \mathfrak{Y}$ for which the induced morphism $\mathfrak{Y}^{\prime} \rightarrow \mathfrak{X}$ is strictly poly-stable.

For example, any smooth morphism is strictly poly-stable. To see that any (strictly) poly-stable morphism is (strictly) pluri-nodal, it suffices to consider a morphism of the form $\operatorname{Spf}(B) \rightarrow \operatorname{Spf}(A)$ with $B=A\left\{T_{0}, \ldots, T_{n}\right\} /$ $\left(T_{0} \cdot \ldots \cdot T_{n}-a\right)$ and $n \geq 2$, and to notice that $B=C\left\{T_{0}, T_{1}\right\} /\left(T_{0} T_{1}-u\right)$ for $C=A\left\{u, T_{2}, \ldots, T_{n}\right\} /\left(u T_{2} \ldots T_{n}-a\right)$. For a formal scheme $\mathfrak{S}$, a locally finitely presented morphism of formal schemes over $\mathfrak{S}$, which is locally isomorphic in the étale topology to the projection $\mathfrak{X} \times \mathfrak{S} \mathfrak{Y} \rightarrow \mathfrak{X}$ where $\mathfrak{Y}$ is poly-stable over $\mathfrak{S}$, will be called trivially poly-stable (with respect to $\mathfrak{S}$ ).

Definition 1.3. Let $\mathfrak{S}$ be a formal scheme. A (strictly) poly-stable fibration over $\mathfrak{S}$ of length $l$ is a sequence of (strictly) poly-stable morphisms

$$
\underline{\mathfrak{X}}=\left(\mathfrak{X}_{l} \stackrel{f_{l-1}}{\longrightarrow} \ldots \stackrel{f_{1}}{\longrightarrow} \mathfrak{X}_{1} \stackrel{f_{0}}{\longrightarrow} \mathfrak{X}_{0}=\mathfrak{S}\right) .
$$

A morphism $\varphi: \underline{\mathfrak{X}}^{\prime} \rightarrow \underline{\mathfrak{X}}$ is a family of morphisms $\varphi_{i}: \mathfrak{X}_{i}^{\prime} \rightarrow \mathfrak{X}_{i}, 0 \leq i \leq l$, such that $\varphi_{0}=1_{\mathfrak{S}}$ and $f_{i-1} \circ \varphi_{i}=\varphi_{i-1} \circ f_{i-1}^{\prime}$ for all $1 \leq i \leq l$. If the morphisms $\varphi_{i}, 1 \leq i \leq l$, possess a property $P, \varphi$ is said to possess the property $P$.

An example of a pluri-stable fibration of schemes is a $G$-pluri nodal fibration from [deJ3, 5.8].

We now specify schemes and formal schemes which will be considered in the paper. First of all, most of the schemes we consider are locally finitely presented over a field $K$ in the above sense. It is more natural to call them schemes of locally finite type over $K$. Note that these are schemes of locally finite type over $K$ in the usual sense with the above additional property, and the latter is automatically satisfied by those of them which are separated.

Furthermore, let $k$ be a non-Archimedean field, i.e., a field complete with respect to a non-Archimedean valuation with values in $\mathbf{R}_{+}$. (The valuation on $k$ is not assumed to be nontrivial). Let $k^{\circ}$ be the ring of integers of $k$, $k^{\circ \circ}$ the maximal ideal of $k^{\circ}$, and $\widetilde{k}=k^{\circ} / k^{\circ \circ}$ the residue field of $k$. The category of formal schemes locally finitely presented over $k^{\circ}$ is denoted by $k^{\circ}-\mathcal{F} s c h$. A formal scheme $\mathfrak{X}$ from $k^{\circ}-\mathcal{F} s c h$ has the closed fibre $\mathfrak{X}_{s}$, 
which is a scheme of locally finite type over $\widetilde{k}$, and the generic fibre $\mathfrak{X}_{\eta}$, which is a paracompact strictly $k$-analytic space, and there is a reduction map $\pi: \mathfrak{X}_{\eta} \rightarrow \mathfrak{X}_{s}($ see $[\operatorname{Ber} 3, \S 1])$. If $\mathfrak{X}=\operatorname{Spf}(A)$, then $\mathfrak{X}_{s}=\operatorname{Spec}(\widetilde{A})$, where $\tilde{A}=A / k^{\circ \circ} A$, and $\mathfrak{X}_{\eta}=\mathcal{M}(\mathcal{A})$, where $\mathcal{A}=A \otimes_{k^{\circ}} k$. One also sets $\mathcal{A}^{\circ}=\left\{\left.f \in \mathcal{A}|| f\right|_{\text {sup }} \leq 1\right\}$, where $|f|_{\text {sup }}=\sup _{x \in \mathcal{M}(\mathcal{A})}|f(x)|, \mathcal{A}^{\circ \circ}=$ $\left\{\left.f \in \mathcal{A}|| f\right|_{\text {sup }}<1\right\}$ and $\widetilde{\mathcal{A}}=\mathcal{A}^{\circ} / \mathcal{A}^{\circ \circ}$. We remark that the image of $A$ in $\mathcal{A}$ is contained in $\mathcal{A}^{\circ}$ and that $\mathcal{A}^{\circ}$ is integral over this image. We also remark that the rings $A$ and $\mathcal{A}^{\circ}$ are separated and complete in the $a$-adic topology, where $a$ is an element of $k^{\circ \circ}$ non-zero if the valuation on $k$ is nontrivial. Notice that, if the valuation on $k$ is trivial, the correspondences $\mathfrak{X} \mapsto \mathfrak{X}_{\eta}$ and $\mathfrak{X} \mapsto \mathfrak{X}_{s}$ are equivalences between the category $k^{\circ}-\mathcal{F} s c h$ and the categories of paracompact strictly $k$-analytic spaces and of schemes of locally finite type over $k$, respectively.

We shall use in the sequel the following fact that follows from the local description of étale morphisms of schemes (see [Ber3, §2]): given an étale morphism $\mathfrak{Y} \rightarrow \mathfrak{X}=\operatorname{Spf}(A)$, each point of $\mathfrak{Y}$ has an open neighborhood isomorphic over $\mathfrak{X}$ to $\operatorname{Spf}(B)$ with $B=C_{\{c\}}, C=A[T] /(P)$ and $c \in C$, where $P(T)$ is a monic polynomial in $A[T]$ such that the image of the derivative $P^{\prime}(T)$ in $B$ is invertible.

A formal scheme $\mathfrak{X}$ from $k^{\circ}-\mathcal{F}$ sch is said to be smooth (resp. (strictly) pluri-nodal, resp. (strictly) poly-stable, resp. trivially poly-stable) if the canonical morphism $\mathfrak{X} \rightarrow \operatorname{Spf}\left(k^{\circ}\right)$ possesses the corresponding property. Notice that if a scheme $\mathcal{X}$ over $k^{\circ}$ possesses one of the above properties, then its formal completion $\widehat{X}$ along the closed fibre $\mathcal{X}_{s}$ possesses the same property.

For $n \geq 1$ and $a \in k^{\circ}$, we set $\mathfrak{T}(n, a)=\operatorname{Spf}\left(k^{\circ}\left\{T_{0}, \ldots, T_{n}\right\} /\left(T_{0} \ldots T_{n}-a\right)\right)$ and, for $m \geq 0$, we set $\mathfrak{S}(m)=\operatorname{Spf}\left(k^{\circ}\left\{S_{1}, \ldots, S_{m}, \frac{1}{S_{1}}, \ldots, \frac{1}{S_{m}}\right\}\right)$. If $|a|=1$, then $\mathfrak{T}(n, a) \stackrel{\sim}{\rightarrow} \mathfrak{S}(n)$. Given two tuples $\mathbf{n}=\left(n_{0}, \ldots, n_{p}\right) \in \mathbf{Z}^{p+1}$ and $\mathbf{a}=\left(a_{0}, \ldots, a_{p}\right) \in k^{p+1}$ with $n_{i} \geq 1$ and $\left|a_{i}\right|<1$, we set $\mathfrak{T}(\mathbf{n}, \mathbf{a})=$ $\mathfrak{T}\left(n_{0}, a_{0}\right) \times \cdots \times \mathfrak{T}\left(n_{p}, a_{p}\right)$. A formal scheme of the form $\mathfrak{S}(m)$ or $\mathfrak{T}(\mathbf{n}, \mathbf{a}) \times$ $\mathfrak{S}(m)$ will be called standard. To consider the above formal schemes simultaneously, we allow the pair of tuples with $p=n_{0}=0$ and $a_{0}=1$ and set $\mathfrak{T}(0,1)=\operatorname{Spf}\left(k^{\circ}\right)$.

To have the possibility to consider automorphisms of formal schemes which act nontrivially on the ground ring, we introduce a category $\mathcal{F} s c h$ whose objects are pairs $(k, \mathfrak{X})$, where $k$ is a non-Archimedean field and $\mathfrak{X}$ is from $k^{\circ}-\mathcal{F} s c h$, and morphisms $(K, \mathfrak{Y}) \rightarrow(k, \mathfrak{X})$ are pairs consisting of an isometric embedding $k \hookrightarrow K$ and a morphism $\mathfrak{Y} \rightarrow \mathfrak{X} \widehat{\otimes}_{k^{\circ}} K^{\circ}$ in $K^{\circ}-\mathcal{F}$ sch . The category $\mathcal{F} s c h$ is a fibred one over the category of non-Archimedean fields, and the correspondence $\mathfrak{X} \mapsto \mathfrak{X}_{\eta}$ gives rise to a functor from $\mathcal{F}$ sch to the similar category $\mathcal{A} n$ of analytic spaces. Furthermore, we denote by $\mathcal{F} s c h^{\text {ét }}\left(\right.$ resp. $\mathcal{F} s c h^{\mathrm{sm}}$, resp. $\left.\mathcal{F} s c h^{\mathrm{tps}}\right)$ the category with the same family of objects as $\mathcal{F} s c h$ but with the morphisms $(K, \mathfrak{Y}) \rightarrow(k, \mathfrak{X})$ for which the morphism $\mathfrak{Y} \rightarrow \mathfrak{X} \widehat{\otimes}_{k^{\circ}} K^{\circ}$ is étale (resp. smooth, resp. trivially poly- 
stable). We also denote by $k^{\circ}-\mathcal{P} s t f, \mathcal{P} s t f, \mathcal{P} s t f^{\text {ét }}, \mathcal{P} s t f^{\mathrm{sm}}$ and $\mathcal{P} s t f^{\mathrm{tps}}$ the full subcategories of $k^{\circ}-\mathcal{F} s c h, \mathcal{F} s c h, \mathcal{F} s c h^{\mathrm{et}}, \mathcal{F} s c h^{\mathrm{sm}}$ and $\mathcal{F} s c h^{\mathrm{tps}}$, respectively, consisting of poly-stable formal schemes. For brevity the pair $(k, \mathfrak{X})$ will be denoted by $\mathfrak{X}$.

The category of poly-stable fibrations over $k^{\circ}$ of length $l$ will be denoted by $k^{\circ}-\mathcal{P} s t f_{l}$. For any bigger non-Archimedean field $K$ there is the evident ground field extension functor $\underline{\mathfrak{X}} \mapsto \mathfrak{X} \widehat{\otimes} K$. We denote by $\mathcal{P} s t f_{l}$ the category of the pairs $(k, \underline{\mathfrak{X}})$ with $\underline{\mathfrak{X}} \in \mathrm{Ob}\left(k^{\circ}-\mathcal{P}_{s t} f_{l}\right)$ and whose morphisms $(K, \mathfrak{Y}) \rightarrow$ $(k, \underline{\mathfrak{X}})$ are the pairs consisting of an isometric embedding $k \hookrightarrow \bar{K}$ and a morphism $\mathfrak{Y} \rightarrow \underline{\mathfrak{X}} \widehat{\otimes} K$ in $K^{\circ}-\mathcal{P} s t f_{l}$. We also denote by $\mathcal{P} s t f_{l}^{\text {ét }}$ (resp. $\mathcal{P} s t f_{l}^{\mathrm{sm}}$, resp. $\mathcal{P} s t f_{l}^{\mathrm{tps}}$ ) the category with the same family of objects as $\mathcal{P} s t f$ but whose morphisms are the pairs for which the morphism $\mathfrak{Y} \rightarrow \mathfrak{X} \widehat{\otimes} K$ is étale (resp. smooth, resp. trivially poly-stable). For brevity, the pair $(k, \underline{\mathfrak{X}})$ will be denoted by $\underline{\mathfrak{x}}$.

Finally, we introduce similar categories of poly-stable schemes and of poly-stable fibrations of schemes over fields and use for them similar notations $\mathcal{P} s t, \mathcal{P} s t^{\text {ét }}, \mathcal{P} s t_{l}$ and so on.

Proposition 1.4. Let $\mathfrak{X}=\operatorname{Spf}(A)$ be a pluri-nodal affine formal scheme over $k^{\circ}$. Then $A \stackrel{\sim}{\rightarrow} \mathcal{A}^{\circ}, \widetilde{A} \stackrel{\sim}{\rightarrow} \widetilde{A}$ and $|\mathcal{A}|_{\text {sup }}=|k|$.

Proof. We remark that the property $\widetilde{A} \stackrel{\sim}{\rightarrow} \widetilde{A}$ trivially follows from the other two properties. Furthermore, if the valuation on $k$ is trivial, then all of the properties are evidently true for an arbitrary $A$. Assume therefore that the valuation on $k$ is nontrivial, and let $\alpha$ be a fixed non-zero element of $k^{\circ \circ}$.

First of all, it suffices to prove the statement for strictly pluri-nodal formal schemes. Indeed, assume that it is true for those formal schemes, and let $\operatorname{Spf}(B) \rightarrow \operatorname{Spf}(A)$ be a surjective étale morphism with strictly pluri-nodal $\operatorname{Spf}(B)$. Then $\operatorname{Spf}\left(B \widehat{\otimes}_{A} B\right)$ is also strictly pluri-nodal, and the necessary properties of $A$ are deduced from those of $B$ and $B \widehat{\otimes}_{A} B$ using the exact sequences $0 \rightarrow A \rightarrow B \rightarrow B \widehat{\otimes}_{A} B$ and $0 \rightarrow \mathcal{A}^{\circ} \rightarrow \mathscr{B}^{\circ} \rightarrow\left(\mathscr{B} \widehat{\otimes}_{\mathcal{A}} \mathcal{B}\right)^{\circ}$.

Thus, assume that $\mathfrak{X}$ is strictly pluri-nodal. We prove the statement by induction on $\operatorname{dim}\left(\mathfrak{X}_{s}\right)$. If $\operatorname{dim}\left(\mathfrak{X}_{s}\right)=0$, then $A$ is a direct product of a finite number of the ring of integers of finite unramified extensions of $k$ and, therefore, it evidently possesses the required properties. Furthermore, let $B=A\left\{T_{0}, T_{1}\right\} /\left(T_{0} \cdot T_{1}-a\right), a \in A$, and assume that $A \stackrel{\sim}{\rightarrow} \mathcal{A}^{\circ}$ and

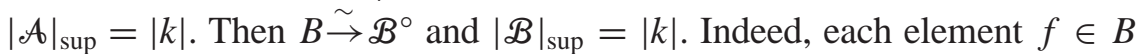
has a unique representation in the form $\sum_{n=-\infty}^{\infty} a_{n} u_{n}$, where $a_{n} \rightarrow 0$ in the $\alpha$-adic topology of $A$ and $u_{n}=T_{0}^{-n}$ for $n<0$ and $u_{n}=T_{1}^{n}$ for $n \geq 0$, and one has $|f|_{\text {sup }}=\max _{n}\left|a_{n}\right|_{\text {sup }}$. Furthermore, each element $f \in \mathscr{B}$ has a unique representation in the above form with $a_{n} \in \mathcal{A}$ such that there exists $\beta \in k^{\circ}$ with $\beta a_{n} \in A$ and $\beta a_{n} \rightarrow 0$ in the $\alpha$-adic topology of $A$. This implies the required properties of $B$.

Assume now that $\mathfrak{Y}=\operatorname{Spf}(B)$ is étale over $\mathfrak{X}=\operatorname{Spf}(A)$ and that the statement is true for $\mathfrak{X}$ and for the formal schemes of smaller dimension. 
The claim is that $B \stackrel{\sim}{\rightarrow} \mathscr{B}^{\circ}$ and $|\mathscr{B}|_{\text {sup }}=|k|$. We prove this in several steps by induction on the number of irreducible components of $\mathfrak{X}_{\eta}=\mathcal{M}(\mathcal{A})$.

Step 1. If $\mathfrak{X}_{\eta}$ is irreducible, then $A$ is integrally closed. It suffices to verify that $\mathcal{A}$ is integrally closed. Indeed, let $f$ be an element of the fraction field of $A$ integral over $A$. Since $\mathcal{A}$ is integrally closed, $f \in \mathcal{A}$. If $f^{n}+$ $a_{1} f^{n-1}+\cdots+a_{n}=0$ is an integral equation of $f$ over $A$, then $|f|_{\text {sup }}^{n} \leq$ $\max _{1 \leq i \leq n-1}\left(|f|_{\text {sup }}^{i}, 1\right)$ and, therefore, $|f|_{\text {sup }} \leq 1$, i.e., $f \in \mathcal{A}^{\circ}=A$.

Since $\mathfrak{X}_{\eta}$ is irreducible, the integral closeness of $\mathcal{A}$ is equivalent to the fact that the local ring $\mathcal{O}_{\mathfrak{X}_{\eta}, x}$ of each point $x \in \operatorname{Max}(\mathcal{A}) \subset \mathfrak{X}_{\eta}$ is integrally closed. The latter fact is local with respect to the étale topology of $\mathfrak{X}$. Thus, it suffices to prove that if a strictly $k$-affinoid space $X=\mathcal{M}(\mathcal{A})$ is irreducible and normal, then the strictly $k$-affinoid space $Y=\mathcal{M}(\mathscr{B})$ with $\mathscr{B}=\mathcal{A}\{u, v\} /(u v-a)$ and $a \neq 0$ is also irreducible and normal. For this we need the following simple fact which will also be used in the next section.

Lemma 1.5. Let $\mathcal{X}=\operatorname{Spec}(A)$ and $\mathcal{Y}=\operatorname{Spec}(B)$, where $A$ is an excellent ring and $B=A[u, v] /(u v-a)$. Then $y$ is normal at a point $y$ if and only if (a) $X$ is normal at the point $x=\varphi(y)$ and $(b) u(y) \neq 0$, or $v(y) \neq 0$, or the image of $a$ in $\mathcal{O}_{x, x}$ is not zero.

Proof. Assume first that $y$ is contained in the normality locus Nor $(y)$ of $y$. Then $x \in \operatorname{Nor}(\mathcal{X})$ because the local ring $\mathcal{O}_{y, y}$ is faithfully flat over $\mathcal{O}_{X, x}$. Suppose that $u(y)=v(y)=0$ and that the image of $a$ in $\mathcal{O}_{X, x}$ is zero. We can shrink $\mathcal{X}$ and assume that $a=0$. In this case $B=A[u, v] /(u v)$ and the point $y$ belongs to at least two irreducible components of $y$.

Assume now that (a) and (b) are true. If $u(y) \neq 0$ or $v(y) \neq 0$, then $\varphi$ is smooth at $y$ and, therefore, $y \in \operatorname{Nor}(y)$. Assume that the image of $a$ in $\mathcal{O}_{X, x}$ is not zero. Then we can shrink $\mathcal{X}$ and assume that $A$ is integrally closed and $a \neq 0$. Each element $f \in B$ has a unique representation in the form $\sum_{i=0}^{\infty} a_{i} u^{i}+\sum_{j=1}^{\infty} b_{j} v^{j}$. One has $u f=\sum_{i=0}^{\infty} a_{i} u^{i+1}+\sum_{j=1}^{\infty} a b_{j} v^{j-1}$, and since $a \neq 0$ the element $u$ is not a zero-divisor in $B$. In particular, $B$ is embedded in $B\left[\frac{1}{u}\right] \stackrel{\sim}{\rightarrow} A\left[u, \frac{1}{u}\right]$. It follows that $B$ is integral. Furthermore, since $B\left[\frac{1}{u}\right]$ is smooth over $A$, it is integrally closed, and to prove the lemma it suffices to verify that any element $f \in B\left[\frac{1}{u}\right]$ integral over $A$ and of the form $\frac{b}{u}, b \in B$, is contained in $B$. Let $f^{n}+b_{1} f^{n-1}+\cdots+b_{n}=0$ be an integral equation of $f$ over $B$. The equation implies that $b^{n} \in u B$. Since $B / u B \stackrel{\sim}{\rightarrow} A[v]$ is reduced, it follows that $b \in u B$.

We apply the lemma to $\mathcal{X}=\operatorname{Spec}(\mathcal{A})$ and $\mathcal{Y}=\operatorname{Spec}(A[u, v] /(u v-a))$. It follows that $y$ is normal. Then from [Ber7, Lemma 3.6], it follows that the strictly $k$-analytic space $y^{\text {an }}$ is normal. Since $Y=\mathcal{M}(\mathscr{B})$ is an affinoid domain in $y^{\text {an }}$, it follows that $Y$ is normal. Since all of the fibres of the morphism $Y \rightarrow X$ are connected, it follows that $Y$ is also connected and, therefore, it is irreducible because it is normal. 
Step 2. The claim is true if $\mathfrak{X}_{\eta}$ is irreducible and $B=C_{\{c\}}, C=$ $A[T] /(P), c \in C$, where $P$ is a monic polynomial in $A[T]$ for which the image of $P^{\prime}(T)$ in $B$ is invertible. By Step $1, A$ is integrally closed. Furthermore, we may assume that $\operatorname{Spec}(\widetilde{B})$ is connected and that the degree of $P$ is minimal over all representations of $B$ in such a form.

(1) The polynomial $P$ is irreducible over the fraction field of $A$. Let $P=P_{1} P_{2}$ be a representation of $P$ as a product of monic polynomials of smaller degrees over the fraction field of $A$. Since $A$ is integrally closed, it follows that $P_{1}, P_{2} \in A[T]$. Let $b$ denote the image of $T$ in $B$. Then $\widetilde{P}_{1}(\widetilde{b}) \widetilde{P}_{2}(\widetilde{b})=0$ and the element $\widetilde{P}_{1}^{\prime}(\widetilde{b}) \widetilde{P}_{2}(\widetilde{b})+\widetilde{P}_{1}(\widetilde{b}) \widetilde{P}_{2}^{\prime}(b)$ is invertible in $\widetilde{B}$. Since $\widetilde{B}$ is étale over the reduced ring $\widetilde{A}$, it is also reduced. It follows that, given an irreducible component $Y$ of $\operatorname{Spec}(\widetilde{B})$, one has either $\left.\widetilde{P}_{1}(\widetilde{b})\right|_{Y}=0$ or $\left.\widetilde{P}_{2}(\widetilde{b})\right|_{Y}=0$. Assume, for example, that $\left.\widetilde{P}_{1}(\widetilde{b})\right|_{Y}=0$. Then the elements $\left.\widetilde{P}_{1}^{\prime}(\widetilde{b})\right|_{Y}$ and $\left.\widetilde{P}_{2}(\widetilde{b})\right|_{Y}$ are invertible. Since $\operatorname{Spec}(\widetilde{B})$ is connected, it follows that the elements $\widetilde{P}_{1}^{\prime}(\widetilde{b})$ and $\widetilde{P}_{2}(\widetilde{b})$ are invertible in $\widetilde{B}$ and, therefore, $P_{1}^{\prime}(b)$ and $P_{2}(b)$ are invertible in $B$. In particular, $P_{1}(b)=0$. If $D=A[T] /\left(P_{1}\right)$, then the canonical epimorphism $C \rightarrow D$ induces an isomorphism $D_{\{d\}} \stackrel{\sim}{\rightarrow} B$, where $d$ is the image of $c$ in $D$, and we can replace $P$ by the polynomial $P_{1}$ of smaller degree. The claim follows. We remark that since the image of $P^{\prime}(T)$ in $B$ is invertible, it follows that the polynomial $P(T)$ is separable over the fraction field of $A$.

(2) The homomorphism $B \rightarrow \mathscr{B}^{\circ}$ is bijective. First of all, since $B$ is flat over $A$, the homomorphism considered is injective. Furthermore, since the both rings are separated and complete in the $\alpha$-adic topology, to prove the surjectivity, it suffices to verify that $B$ is dense in $\mathscr{B}^{\circ}$. A dense subset in $\mathscr{B}$ is provided by elements of the form $\sigma(f) / \sigma(c)^{n}$, where $\sigma$ is the canonical homomorphism $\mathcal{C}=C \otimes_{k^{\circ}} k \rightarrow \mathscr{B}$ and $f \in \mathcal{C}$. Let $\sigma(f) / \sigma(c)^{n}$ be such an element of $\mathscr{B}^{\circ}$, i.e., $\left|\sigma(f) / \sigma(c)^{n}\right|_{\text {sup }} \leq 1$. We claim that $\sigma(f) / \sigma(c)^{n} \in B$. Indeed, since $|\sigma(c)(y)|=1$ for all points $y \in \mathfrak{Y}_{\eta}$, it follows that $|\sigma(f)|_{\text {sup }} \leq 1$. By [BGR, 7.2.6/2], one has $|\sigma(f)|_{\text {sup }}=\left|c^{m} f\right|_{\text {sup }}$ for a sufficiently large $m$. Replacing $f$ by $c^{m} f$, we may assume that $|f|_{\text {sup }} \leq 1$, i.e. $f \in \mathcal{C}^{\circ}$, and since $\sigma(c) \in B^{*}$ it suffices to show that $\sigma(f) \in B=\bar{C}_{\{c\}}$. By [BGR, 6.3.5/1], the ring $\mathcal{C}^{\circ}$ is integral over $A=\mathcal{A}^{\circ}$. It follows that $\mathcal{C}^{\circ}$ is contained in the integral closure of $A$ in $L=C \otimes_{A} K$, where $K$ is the fraction field of $A$. Since $L$ is separable over $K$, it follows that $f=g / P^{\prime}(\gamma)$ for some $g \in C$, where $\gamma$ is the image of $T$ in $C$. Since the image of $P^{\prime}(\gamma)$ in $B$ is invertible, it follows that $\sigma(f) \in B$.

(3) The homomorphism $\widetilde{B} \rightarrow \widetilde{\mathscr{B}}$ is bijective. By (2), it suffices to verify that the homomorphism is injective. Let $g$ be an element of $B$ with $|g|_{\text {sup }}<1$. We have to verify that $g \in k^{\circ 0} B$. For this we can replace $g$ by an element of the form $\sigma(f) / \sigma(c)^{n}$. As above, replacing $f$ by $c^{m} f$ for a sufficiently large $m$, we may assume that $|f|_{\text {sup }}<1$, i.e., $f \in \mathcal{C}^{\circ \circ}$. Let $f^{d}+a_{1} f^{d-1}+$ $\cdots+a_{d}=0$ be the minimal equation of $f$ over $\mathcal{A}$. By [BGR, 3.8.1/7], one has $|f|_{\text {sup }}=\min _{i}\left|a_{i}\right|^{1 / i}$. It follows that $a_{i} \in \mathcal{A}^{\circ 0}=k^{\circ \circ} A$. The latter implies 
that $f^{d} \in k^{\circ \circ} C$, and therefore $\sigma(f)^{d} \in k^{\circ \circ} B$. Since the ring $\widetilde{B}$ is reduced, it follows that $\sigma(f) \in k^{\circ \circ} B$ and therefore $g \in k^{\circ \circ} B$.

(4) One has $|\mathscr{B}|_{\text {sup }}=|k|$. For this we use the following observation.

Lemma 1.6. Let $\mathfrak{Y} \rightarrow \mathfrak{X}$ be an étale morphism of formal schemes locally finitely presented over $k^{\circ}$. Then for any point $y \in \mathfrak{Y}_{\eta}$ the field $\mathscr{H}(y)$ is a finite unramified extension of $\mathcal{H}(x)$, where $x$ is the image of $y$ in $\mathfrak{X}_{\eta}$.

The statement and its proof work also for special formal schemes as defined in [Ber6, §1] over $k^{\circ}$ with a discrete valuation (not necessarily nontrivial).

Proof. We may assume that $\mathfrak{X}=\operatorname{Spf}(A)$ and $\mathfrak{Y}=\operatorname{Spf}(B)$, where $B=C_{\{c\}}$, $C=A[T] /(P)$, and $P \in A[T]$ is a monic polynomial such that the image of $P^{\prime}(T)$ in $B$ is invertible. Since the morphism $\mathfrak{Y}_{\eta} \rightarrow \mathfrak{X}_{\eta}$ is quasi-étale, $\mathscr{H}(y)$ is a finite separable extension of $\mathscr{H}(x)$ generated by the image $\beta$ of $T$ in $\mathscr{H}(y)$. Let $Q(\beta)=0$ be the minimal monic equation of $\beta$ over $\mathscr{H}(x)$. One has $Q \in \mathscr{H}(x)^{\circ}[T]$. Since $P(\beta)=0$, it follows that $P=Q R$ for some $R \in \mathscr{H}(x)^{\circ}[T]$, and therefore $Q^{\prime}(\beta)$ is invertible in $\mathscr{H}(y)^{\circ}$. This immediately implies that $\mathscr{H}(y)^{\circ}=\mathscr{H}(x)^{\circ}[\beta]$ and that $\mathscr{H}(y)^{\circ}$ is étale over $\mathscr{H}(x)^{\circ}$, i.e., $\mathscr{H}(y)$ is unramified over $\mathscr{H}(x)$.

By [Ber1, 2.4.4], for every $f \in \mathscr{B}$ one has $|f|_{\text {sup }}=\max _{y \in \Gamma(\mathcal{B})}|f(x)|$, where $\Gamma(\mathscr{B})$ is the Shilov boundary of $\mathscr{B}$, and $\Gamma(\mathscr{B})$ coincides with the preimage of the set of generic points of the irreducible components of $\mathfrak{Y}_{s}=\operatorname{Spec}(\widetilde{B})$ under the reduction map $\mathfrak{Y}_{\eta}=\mathcal{M}(\mathscr{B}) \rightarrow \mathfrak{Y}_{s}$. Recall also that the preimage of such a generic point consists of one point. By (3), $\widetilde{B}=\widetilde{B}$, and therefore the generic points of $\mathfrak{Y}_{s}$ are the preimages of the generic points of $\mathfrak{X}_{s}=\operatorname{Spec}(\widetilde{A})$. It follows that $\Gamma(\mathscr{B})$ is the preimage of $\Gamma(\mathcal{A})$ under the map $\mathfrak{Y}_{\eta} \rightarrow \mathfrak{X}_{\eta}=\mathcal{M}(\mathcal{A})$. Since $|\mathcal{H}(x)|=|k|$ for all $x \in \Gamma(\mathcal{A})$, Lemma 1.6 implies that $|\mathcal{H}(y)|=|k|$ for all $y \in \Gamma(\mathscr{B})$, and therefore $|\mathscr{B}|_{\text {sup }}=|k|$.

Step 3. The claim is true if $\mathfrak{X}_{\eta}$ is irreducible. Indeed, we can find a finite covering of $\mathfrak{Y}$ by open affine subschemes $\operatorname{Spf}\left(B_{i}\right)$, where $B_{i}$ are of the form from Step 2, and for each pair $i, j$ a finite open covering of $\operatorname{Spf}\left(B_{i}\right) \cap \operatorname{Spf}\left(B_{j}\right)$ by open affine subschemes $\operatorname{Spf}\left(B_{i j l}\right)$, where $B_{i j l}$ are also of the form from Step 2 . Then the necessary properties of $B$ are established using the exact sequences $0 \rightarrow B \rightarrow \prod_{i} B_{i} \rightarrow \prod_{i j l} B_{i j l}$ and $0 \rightarrow \mathscr{B}^{\circ} \rightarrow \prod_{i} \mathscr{B}_{i}^{\circ} \rightarrow$ $\prod_{i j l} \mathscr{B}_{i j l}^{\circ}$.

Step 4. The claim is true if $\mathfrak{Y}$ is an open subscheme of $\mathfrak{X}$. Indeed, by the reasoning from Step 3, it suffices to consider the case $B=A_{\{f\}}$, where $f \in A \backslash k^{\circ \circ} A$. In this case we use the argument from the substep (2) of Step 2. Namely, it suffices to verify that $B$ is dense in $\mathscr{B}^{\circ}$. Let $\sigma$ denote the canonical homomorphism $\mathcal{A} \rightarrow \mathscr{B}$, and let $\sigma(a) / \sigma(f)^{n}, a \in A$ be an element of $\mathcal{B}$ with $\left|\sigma(a) / \sigma(f)^{n}\right|_{\text {sup }} \leq 1$. Since $|\sigma(f)(y)|=1$ for all $y \in \mathfrak{Y}_{\eta}$, it follows 
that $|\sigma(a)|_{\text {sup }} \leq 1$. By [BGR, 7.2.6/2], one has $|\sigma(a)|_{\text {sup }}=\left|f^{m} a\right|_{\text {sup }}$ for a sufficiently large $m$, i.e., $f^{m} a \in \mathcal{A}^{\circ}=A$. It follows that $\sigma(a) / \sigma(f)^{n} \in B$.

Step 5. The claim is true in the general case. By Step 4, the claim is local with respect to $\mathfrak{X}$. Hence, we can shrink $\mathfrak{X}$ and assume that the morphism $\mathfrak{X} \rightarrow \operatorname{Spf}\left(k^{\circ}\right)$ is a composition of étale morphisms with morphisms of the form $\operatorname{Spf}(D) \rightarrow \operatorname{Spf}(C)$ with $D=C\{u, v\} /(u v-c)$. If all of the elements $c$ from the latter morphisms are not zero, then Step 1 implies that $\mathfrak{X}_{\eta}$ is normal, i.e., we are in the situation of that step. Assume that $\mathfrak{X}_{\eta}$ is not normal. Then for some intermediate morphism $\operatorname{Spf}(D) \rightarrow \operatorname{Spf}(C)$ one has $D=C\{u, v\} /(u v)$. We set $\mathfrak{X}_{1}=\operatorname{Spf}(A / u A), \mathfrak{X}_{2}=\operatorname{Spf}(A / v A)$ and $\mathfrak{X}_{3}=\operatorname{Spf}(A /(u A+v A))$. These are strictly pluri-nodal affine formal schemes over $k^{\circ}$. The numbers of irreducible components of $\mathfrak{X}_{1, \eta}$ and $\mathfrak{X}_{2, \eta}$ are strictly less than that of $\mathfrak{X}_{\eta}$, and the dimension of $\mathfrak{X}_{3, s}$ is strictly less than that of $\mathfrak{X}_{s}$. By induction, the rings $B_{1}=B / u B, B_{2}=B / v B$ and $B_{3}=B /(u B+v B)$ possess the required properties. Since $B$ is flat over $D$, it follows that there is an exact sequence $0 \rightarrow B \rightarrow B_{1} \times B_{2} \rightarrow B_{3}$. The required properties of $B$ are now deduced using it and the exact sequence $0 \rightarrow \mathscr{B} \rightarrow \mathscr{B}_{1} \times \mathscr{B}_{2} \rightarrow \mathscr{B}_{3}$.

Corollary 1.7. Let $\mathfrak{X}$ be a pluri-nodal formal scheme over $k^{\circ}$, and let gen $\left(\mathfrak{X}_{s}\right)$ denote the set of generic points of the irreducible components of $\mathfrak{X}_{s}$. Then

(i) the reduction map $\mathfrak{X}_{\eta} \rightarrow \mathfrak{X}_{s}$ is surjective;

(ii) for any $\mathbf{x} \in \operatorname{gen}\left(\mathfrak{X}_{s}\right)$, there exists a unique point $x \in \mathfrak{X}_{\eta}$ with $\pi(x)=\mathbf{x}$, and one has $\widetilde{k}(\mathbf{x}) \stackrel{\sim}{\rightarrow} \widetilde{\mathscr{H}(x)}$;

(iii) if $\mathfrak{X}$ is affine, then the set $\pi^{-1}\left(\operatorname{gen}\left(\mathfrak{X}_{s}\right)\right)$ is the Shilov boundary of $\mathfrak{X}_{\eta}$.

Proof. Everything follows from Proposition 1.4 and [Ber1, Propos. 2.2.4].

Corollary 1.8. The generic fibre of a pluri-nodal formal scheme over $k^{\circ}$ is connected if and only if its closed fibre is connected.

\section{A stratification of a pluri-nodal scheme}

Let $\mathcal{X}$ be a scheme of locally finite type over a field $K$. Recall again that it is a scheme which admits a locally finite covering by open affine subschemes of finite type over $K$. It follows that any irreducible component of $\mathcal{X}$ is quasi-compact and has a non-empty intersection with at most finite number of other irreducible components, and the intersection of any family of irreducible components has a finite number of irreducible components. (Of course, if the above intersection is non-empty, the family is finite.) Moreover, any locally closed subset of $\mathcal{X}$, considered as a reduced scheme, is of locally finite type over $K$. The set of irreducible components of $\mathcal{X}$ will be denoted by $\operatorname{irr}(\mathcal{X})$. 
Assume that $\mathcal{X}$ is reduced. Since $\mathcal{X}$ is an excellent scheme [EGAIV, §7], the normality locus $\operatorname{Nor}(\mathcal{X})$ of $\mathcal{X}$ is open and dense in $\mathcal{X}$. We set $\mathcal{X}^{(0)}=\mathcal{X}$ and $\mathcal{X}^{(i+1)}=\mathcal{X}^{(i)} \backslash \operatorname{Nor}\left(\mathcal{X}^{(i)}\right), i \geq 0$. The irreducible components of the locally closed subsets $\mathcal{X}^{(i)} \backslash \mathcal{X}^{(i+1)}=\operatorname{Nor}\left(\mathcal{X}^{(i)}\right)$ will be called the strata of $\mathcal{X}$. (Note that $\operatorname{Nor}\left(\mathcal{X}^{(i)}\right)$ is a disjoint union of its irreducible components.) The stratification of $\mathcal{X}$ constructed in this way is evidently locally finite. A subset which is a union of strata is called a strata subset of $\mathcal{X}$.

Furthermore, we define another filtration $\mathcal{X}=\mathcal{X}_{(0)} \supset \mathcal{X}_{(1)} \supset \ldots$ by the property that $\mathcal{X}_{(i+1)}$ consists of the points of $\mathcal{X}_{(i)}$ which are contained in at least two irreducible components of $\mathcal{X}_{(i)}$. We say that $\mathcal{X}$ is quasinormal if all of the irreducible components of each $\mathcal{X}_{(i)}$ are normal. Notice that the property to be quasi-normal is local with respect to the Zariski topology and, for an étale morphism $y \rightarrow X$ with quasi-normal $\mathcal{X}, \mathcal{y}$ is also quasi-normal. Notice also that if $\mathcal{X}$ is quasi-normal then $\mathcal{X}^{(i)}=\mathcal{X}_{(i)}$ for all $i \geq 0$. Indeed, since the irreducible components of $\mathcal{X}$ are normal, then $\operatorname{Nor}(\mathcal{X})=\mathcal{X} \backslash \mathcal{X}_{(1)}$, i.e., $\mathcal{X}^{(1)}=\mathcal{X}_{(1)}$, and the statement for $\mathcal{X}$ is reduced to that for $\mathcal{X}_{(1)}$.

Proposition 2.1. Let $\mathcal{X}$ be a pluri-nodal scheme over $K$. Then

(i) the closure of every stratum of $\mathcal{X}$ is a strata subset of $\mathcal{X}$;

(ii) if $\mathcal{X}$ is strictly pluri-nodal, it is quasi-normal.

Lemma 2.2. Let $\varphi: \mathcal{y} \rightarrow \mathcal{X}$ be an étale morphism between reduced schemes of locally finite type over $K$. Then

(i) $\varphi$ induces an étale morphism from each stratum of $\mathcal{Y}$ to a stratum of $\mathcal{X}$; in particular, the preimage of each stratum of $\mathcal{X}$ is a strata subset of $\mathcal{Y}$;

(ii) if $\mathcal{X}$ is quasi-normal and possesses the property (i) of Proposition 2.1, then $\mathcal{Y}$ possesses the same property;

Assume that $\varphi$ is surjective. Then

(iii) if, for a locally closed subset $\mathcal{X}^{\prime} \subset \mathcal{X}, \varphi^{-1}\left(\mathcal{X}^{\prime}\right)$ is a strata subset of $\mathcal{Y}$, then $\mathcal{X}^{\prime}$ is a strata subset of $\mathcal{X}$;

(iv) if $\mathcal{Y}$ possesses the property (i) of Proposition 2.1, then $\mathcal{X}$ possesses the same property.

Proof. (i) Since $\operatorname{Nor}(\mathcal{Y})=\varphi^{-1}(\operatorname{Nor}(\mathcal{X}))$, the statement is evidently true for the strata of $y$ in $\operatorname{Nor}(\mathcal{Y})$, and is reduced to that for the induced morphism $y^{(1)}=\varphi^{-1}\left(X^{(1)}\right) \rightarrow X^{(1)}$.

(ii) Let $Y$ be a stratum of $\mathcal{y}_{\text {with }} Y \subset \mathcal{y}_{(i)} \backslash y_{(i+1)}, i \geq 0$. Then $\bar{Y}$ is an irreducible component of $\mathcal{Y}_{(i)}$. If $X$ is the stratum of $\mathcal{X}$ that contains $\varphi(Y)$, then $\bar{X}$ is an irreducible component of $\mathcal{X}_{(i)}$. Since $\bar{X}$ is normal and the induced morphism $\varphi^{-1}(\bar{X}) \rightarrow \bar{X}$ is étale, it follows that $\varphi^{-1}(\bar{X})$ is a disjoint union of its irreducible components. One of them is $\bar{Y}$, and the statement follows.

(iii) Since $\varphi$ is surjective, it suffices to verify that, for each stratum $Y$ of $\mathcal{Y}$ in $\varphi^{-1}\left(X^{\prime}\right)$, one has $X \subset \mathcal{X}^{\prime}$, where $X$ is the stratum of $\mathcal{X}$ that 
contains $\varphi(Y)$. By (i), $\varphi$ induces an étale morphism $Y \rightarrow X$ and, in particular, $\varphi(Y)$ is open in $X$. It follows that, for each stratum $Y^{\prime}$ of $\mathcal{Y}$ with $\varphi\left(Y^{\prime}\right) \subset X$, the intersection $\varphi\left(Y^{\prime}\right) \cap \varphi(Y)$ is non-empty. The latter implies that $Y^{\prime} \cap$ $\varphi^{-1}\left(X^{\prime}\right) \neq \emptyset$ and, therefore, $Y^{\prime} \subset \varphi^{-1}\left(\mathcal{X}^{\prime}\right)$ because $\varphi^{-1}\left(\mathcal{X}^{\prime}\right)$ is a union of strata. It follows that $\varphi\left(Y^{\prime}\right) \subset \mathcal{X}^{\prime}$ and, therefore, $X \subset \mathcal{X}^{\prime}$ because $\varphi$ is surjective.

(iv) Let $X$ be a stratum of $X$. The assumption implies that $\overline{\varphi^{-1}(X)}$ is a union of strata. Since $\varphi$ is an open map, one has $\overline{\varphi^{-1}(X)}=\varphi^{-1}(\bar{X})$ and, by (iii), $\bar{X}$ is a union of strata.

Lemma 2.3. Let $\varphi: y=\operatorname{Spec}(B) \rightarrow X=\operatorname{Spec}(A)$ with $B=A[u, v] /(u v-a)$. Assume that $\mathcal{X}$ possesses the property (i) of Proposition 2.1. Then

(i) $y$ possesses the same property;

(ii) given a stratum $X$ of $\mathcal{X}$, one has

(1) if $\left.a\right|_{X} \neq 0$, then $\varphi^{-1}(X)$ is a stratum of $\mathcal{Y}$, and the induced morphism $\varphi^{-1}(X) \rightarrow X$ is flat and generically smooth;

(2) if $\left.a\right|_{X}=0$, then $\varphi^{-1}(X)$ is a union of the two irreducible components $Y^{\prime} \stackrel{\sim}{\rightarrow} X \times \operatorname{Spec}(K[v])$ and $Y^{\prime \prime} \stackrel{\sim}{\rightarrow} X \times \operatorname{Spec}(K[u])$, and $Z:=Y^{\prime} \cap Y^{\prime \prime} \stackrel{\sim}{\rightarrow} X$; moreover, $Y^{\prime} \backslash Z, Y^{\prime \prime} \backslash Z$ and $Z$ are strata of $\mathcal{Y}$;

(iii) if $\mathcal{X}$ is quasi-normal, then so is $\mathcal{Y}$.

Proof. Assume first that $X$ is an irreducible component in $\operatorname{Nor}(\mathcal{X})$. In the case (1), Lemma 1.5 implies that $\frac{\varphi^{-1}(X)}{\varphi^{-1}(X)}$ is irreducible and normal, and all points from its complement in $\overline{\varphi^{-1}(X)}=\varphi^{-1}(\bar{X})$ are not normal, i.e., $\varphi^{-1}(X)$ is an irreducible component of $\operatorname{Nor}(\mathcal{Y})$. (The above equality follows from the fact that $\varphi$ is an open map.) In the case (2), one has $\overline{\varphi^{-1}(X)}=Y^{\prime} \cup$ $Y^{\prime \prime} \cup \varphi^{-1}(\bar{X} \backslash X)$ and, therefore, $Y^{\prime} \backslash Z$ and $Y^{\prime \prime} \backslash Z$ are irreducible components in $\operatorname{Nor}(\mathcal{Y})$, i.e., they are strata of $\mathcal{Y}$. Since $Z \stackrel{\sim}{\rightarrow} X$, the set $Z$ is open in irreducible components of $\operatorname{Nor}\left(\mathcal{Y}_{(1)}\right)$ and of $\operatorname{Nor}\left(\mathcal{y}^{(1)}\right)$. We claim that each point from $\bar{Z} \backslash Z$ is contained in at least two irreducible components of $\varphi^{-1}\left(\mathcal{X}^{(1)}\right)$. (Note that $\bar{Z} \backslash Z \subset \varphi^{-1}(\bar{X} \backslash X)$.) Indeed, let $X^{\prime}$ be an irreducible component of $\mathcal{X}^{(1)}$ that contains the image of a point from $\bar{Z} \backslash Z$. Since $\bar{X}$ is a strata set, it follows that $X^{\prime} \subset \bar{X} \backslash X$ and, therefore, $\left.a\right|_{X^{\prime}}=0$. This implies that $\varphi^{-1}\left(X^{\prime}\right)$ has two irreducible components whose intersection contains the point and which are also irreducible components of $\varphi^{-1}\left(\mathcal{X}^{(1)}\right)$. It follows that $Z$ is an irreducible component of $\operatorname{Nor}\left(\mathcal{y}_{(1)}\right)$ and of $\operatorname{Nor}\left(\mathcal{y}^{(1)}\right)$ and, in particular, $Z$ is a stratum of $\mathcal{Y}$. It follows also that $\left(\mathcal{Y}^{(1)}\right)^{(1)}=\left(\varphi^{-1}\left(\mathcal{X}^{(1)}\right)\right)^{(1)}$, and if $\mathcal{X}^{(1)}=\mathcal{X}_{(1)}$ then $y^{(1)}=\mathcal{Y}_{(1)}$. Thus the statements (ii) and (iii) for $\varphi$ are reduced to those for the morphism $\varphi^{-1}\left(\mathcal{X}^{(1)}\right) \rightarrow \mathcal{X}^{(1)}$. The statement (i) follows from (ii) and the equality $\overline{\varphi^{-1}(X)}=\varphi^{-1}(\bar{X})$.

Proof of Proposition 2.1. Both statements follow straightforwardly from Lemmas 2.2 and 2.3. 
Let $\operatorname{str}(\mathcal{X})$ denote the set of the generic points of strata of $\mathcal{X}$.

Corollary 2.4. Let $X$ be a pluri-nodal scheme over $K$. Then for each stratum $X$ of $\mathcal{X}$ one has $X=\bar{x} \backslash(\cup \bar{y})$, where $x$ is the generic point of $X$ and the union is taken over all points $y \in \operatorname{str}(\mathcal{X})$ with $x \notin \bar{y}$.

The set of strata of a strictly poly-stable scheme can be described as follows.

Proposition 2.5. Let $\mathcal{X}$ be a strictly poly-stable scheme over $K$. Then the intersection of any set of irreducible components of $\mathcal{X}$ is smooth, and the family of strata coincides with the family of irreducible components of sets of the form $\left(\cap_{X \in A} X\right) \backslash\left(\cup_{Y \notin A} Y\right)$, where $A$ is a finite subset of $\operatorname{irr}(\mathcal{X})$.

Proof. Both statements are easily verified for direct products of schemes of the form $\operatorname{Spec}\left(K\left[T_{0}, \ldots, T_{n}\right] /\left(T_{0} \ldots . T_{n}\right)\right)$ and $\operatorname{Spec}\left(K\left[T_{1}, \ldots, T_{n}\right.\right.$, $\left.\left.T_{1}^{-1}, \ldots, T_{n}^{-1}\right]\right)$, and easily follow from this in the general case.

Corollary 2.6. All of the strata of a poly-stable scheme are smooth.

Proposition 2.7. Let $\varphi: \mathcal{Y} \rightarrow \mathcal{X}$ be a pluri-nodal morphism to a plurinodal scheme $\mathcal{X}$ over $K$. Then

(i) the image of a stratum $Y$ of $\mathcal{Y}$ is contained in a stratum $X$ of $\mathcal{X}$, and the induced morphism $Y \rightarrow X$ is flat and generically smooth;

(ii) $\operatorname{str}(y)=\cup_{x \in \operatorname{str}(X)} \operatorname{str}\left(y_{x}\right)$.

Proof. Lemmas 2.2(i) and 2.3 imply that the statements are true if $\varphi$ is of the form $y \stackrel{g}{\rightarrow} X_{n} \stackrel{f_{n-1}}{\rightarrow} \ldots \stackrel{f_{1}}{\rightarrow} X_{1} \stackrel{h}{\rightarrow} X$, where $g$ and $h$ are étale, $X_{i}$ are affine, and $f_{i}$ are étale or of the form considered in Lemma 2.3. It follows that in the general case we can find a surjective étale morphism $\psi: y^{\prime} \rightarrow y$ such that the statements are true for the morphism $\varphi \psi: \mathcal{y}^{\prime} \rightarrow \mathcal{X}$. Let $Y$ be a stratum of $\mathcal{Y}$, and let $X$ be stratum of $\mathcal{X}$ with $\varphi(Y) \cap X \neq \emptyset$. It suffices to verify that $\varphi(Y) \subset X$. For this it suffices to check that $\varphi^{-1}(X)$ is a strata subset of $\mathcal{Y}$. But the latter follows from Lemma 2.2(iii) applied to the subset $\varphi^{-1}(X)$ and the morphism $\psi$.

Notice that, for a pluri-nodal scheme $\mathcal{X}$ over $K$ and a bigger field $K^{\prime}$, the morphism $\mathcal{X} \otimes K^{\prime} \rightarrow \mathcal{X}$ gives rise to a surjective morphism from each stratum of $\mathcal{X} \otimes K^{\prime}$ to a stratum of $\mathcal{X}$ and to a surjective map $\operatorname{str}\left(\mathcal{X} \otimes K^{\prime}\right) \rightarrow$ $\operatorname{str}(\mathcal{X})$.

We introduce a partial ordering on the $\operatorname{set} \operatorname{str}(\mathcal{X})$ as follows: $x \leq y$ if $y \in \bar{x}$. For a point $x \in \operatorname{str}(\mathcal{X})$, we set $\operatorname{str}_{\leq x}(\mathcal{X})=\{y \in \operatorname{str}(\mathcal{X}) \mid y \leq x\}$. Notice that the maps $\operatorname{str}(\mathcal{X}) \rightarrow \operatorname{str}(\mathcal{X})$ from Proposition 2.7 and $\operatorname{str}\left(\mathcal{X} \otimes K^{\prime}\right) \rightarrow$ $\operatorname{str}(\mathcal{X})$ from the previous paragraph are maps of partially ordered sets.

Corollary 2.8. In the situation of Proposition 2.7, if the morphism $\varphi$ is surjective then $\operatorname{Coker}(\operatorname{str}(\mathcal{Y} \times x, y) \stackrel{\rightarrow}{\rightarrow} \operatorname{str}(\mathcal{Y})) \stackrel{\sim}{\rightarrow} \operatorname{str}(X)$. 
Proof. It suffices to check that each pair $x \leq x^{\prime}$ in $\operatorname{str}(\mathcal{X})$ comes from a pair $y \leq y^{\prime}$ in $\operatorname{str}(\mathcal{Y})$. One has $X^{\prime} \subset \bar{X}$ for the corresponding strata of $\mathcal{X}$ and, therefore, $\varphi^{-1}\left(X^{\prime}\right) \subset \varphi^{-1}(\bar{X})=\overline{\varphi^{-1}(X)}$. It follows that for each stratum $Y^{\prime}$ of $y$ over $X^{\prime}$ there exists a stratum $Y$ over $X$ with $Y^{\prime} \cap \bar{Y} \neq \emptyset$ and, therefore, $Y^{\prime} \subset \bar{Y}$, i.e., $y \leq y^{\prime}$ for their generic points.

Proposition 2.9. Let $\varphi: \mathcal{Y} \rightarrow \mathcal{X}$ be a strictly pluri-nodal morphism with strictly pluri-nodal $\mathcal{X}$, and let $x, x^{\prime} \in \operatorname{str}(\mathcal{X})$ be a pair of points with $x^{\prime} \leq x$. Then

(i) for each point $y \in \operatorname{str}(\mathcal{Y})$ with $\varphi(y)=x$, the set of points $y^{\prime} \in \operatorname{str}(\mathcal{Y})$ with $\varphi\left(y^{\prime}\right)=x^{\prime}$ and $y^{\prime} \leq y$ is non-empty and has a unique maximal element; in particular, there is a well defined morphism of partially ordered sets $\operatorname{str}\left(\mathcal{y}_{x}\right) \rightarrow \operatorname{str}\left(\mathcal{Y}_{x^{\prime}}\right)$;

(ii) the above map takes minimal points to minimal points; in particular, there is a well defined map $\operatorname{irr}\left(y_{x}\right) \rightarrow \operatorname{irr}\left(y_{x^{\prime}}\right)$;

(iii) for a point $x^{\prime \prime} \in \operatorname{str}(\mathcal{X})$ with $x^{\prime \prime} \leq x^{\prime}$, the following diagram is commutative

$$
\begin{gathered}
\operatorname{str}\left(y_{x}\right) \longrightarrow \operatorname{str}\left(\mathcal{y}_{x^{\prime}}\right) \\
\searrow \swarrow \\
\operatorname{str}\left(y_{x^{\prime \prime}}\right)
\end{gathered}
$$

Lemma 2.10. Let $\varphi: y \rightarrow X$ be an étale morphism with strictly plurinodal $\mathcal{X}$, and let $y \in \mathcal{Y}$ and $x=\varphi(y)$. Then there is an isomorphism of partially ordered sets $\operatorname{str}_{\leq y}(\mathcal{Y}) \stackrel{\sim}{\rightarrow} \operatorname{str}_{\leq x}(\mathcal{X})$.

Proof. The minimal elements of both sets are exactly the generic points of the irreducible components of $y$ and $\mathcal{X}$ passing through $y$ and $x$, respectively. It follows that $\operatorname{str}_{\leq y}(\mathcal{Y}) \cap \operatorname{Nor}(\mathcal{Y}) \stackrel{\sim}{\rightarrow} \operatorname{str}_{\leq x}(\mathcal{X}) \cap \operatorname{Nor}(\mathcal{X})$ and, therefore, the bijectivity statement for $\varphi$ is reduced to that for the morphism $\mathcal{Y}^{(1)}=\varphi^{-1}\left(\mathcal{X}^{(1)}\right) \rightarrow \mathcal{X}^{(1)}$. It remains to verify that if $\varphi\left(y^{\prime \prime}\right) \leq \varphi\left(y^{\prime}\right)$ for two points $y^{\prime}, y^{\prime \prime} \in \operatorname{str}_{\leq y}(\mathcal{X})$ then $y^{\prime \prime} \leq y^{\prime}$. Let $X^{\prime}$ and $X^{\prime \prime}$ be the strata of $\mathcal{X}$ whose generic points are $\varphi\left(y^{\prime}\right)$ and $\varphi\left(y^{\prime \prime}\right)$. The assumption means that $X^{\prime} \subset \bar{X}^{\prime \prime}$. Since $\bar{X}^{\prime \prime}$ is normal, $\overline{\varphi^{-1}\left(X^{\prime \prime}\right)}=\varphi^{-1}\left(\bar{X}^{\prime \prime}\right)$ is a disjoint union of irreducible components, and $\bar{y}^{\prime \prime}$ is the only one of them that contains $y$. It follows that $y^{\prime \prime} \leq y^{\prime}$.

Proof of Proposition 2.9. We remark that if the statement is true for the morphism $\varphi$ and a morphism $\psi: \mathcal{Z} \rightarrow \mathcal{Z}$ then it is also true for their composition $\varphi \circ \psi$. Thus, since the statement is local with respect to the Zariski topology of $\mathcal{y}$, it suffices to consider the two cases when $\varphi$ is étale or of the form $\operatorname{Spec}(B) \rightarrow \operatorname{Spec}(A)$ with $B=A[u, v] /(u v-a)$. In both cases everything follows straightforwardly from Lemmas 2.10 and 2.3. 
Corollary 2.11. In the situation of Proposition 2.9, let $\psi: Z \rightarrow Y$ be a second strictly pluri-nodal morphism. Then the following diagram is commutative

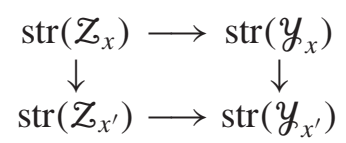

Proof. Let $z^{\prime}$ and $y$ be the images of a point $z \in \operatorname{str}\left(\mathcal{Z}_{x}\right)$ in $\operatorname{str}\left(\mathcal{Z}_{x^{\prime}}\right)$ and $\operatorname{str}\left(\mathcal{y}_{x}\right)$, respectively, and let $y^{\prime}$ and $\tilde{y}^{\prime}$ be the images of $z^{\prime}$ and $y$ in $\operatorname{str}\left(y_{x^{\prime}}\right)$. It is clear that $y^{\prime} \leq \tilde{y}^{\prime}$. On the other hand, let $\widetilde{z}^{\prime}$ be the image of $z$ under the map $\operatorname{str}\left(\mathcal{Z}_{y}\right) \rightarrow \operatorname{str}\left(\mathcal{Z}_{\widetilde{y}^{\prime}}\right)$. One has $\widetilde{z}^{\prime} \leq z$ and $\psi\left(\widetilde{z}^{\prime}\right)=\widetilde{y}^{\prime}$ and, therefore, $\widetilde{z}^{\prime} \in \operatorname{str}\left(\mathcal{Z}_{x^{\prime}}\right)$. It follows that $\widetilde{z}^{\prime} \leq z^{\prime}$. This implies that $\widetilde{y}^{\prime} \leq y^{\prime}$, i.e., $y^{\prime}=\widetilde{y}^{\prime}$.

We say that a strictly pluri-nodal scheme $\mathcal{X}$ is elementary if the partially ordered $\operatorname{set} \operatorname{str}(\mathcal{X})$ has a unique maximal element. Notice that, given a strictly pluri-nodal scheme $\mathcal{X}$ and a point $x \in \mathcal{X}$, any sufficiently small open neighborhood of $x$ is elementary. Indeed, let $x_{0}$ be the generic point of the stratum of $\mathcal{X}$ that contains $x$, and let $\mathcal{U}=\mathcal{X} \backslash(\cup \bar{y})$, where the union is taken over all points $y \in \operatorname{str}(\mathcal{X})$ with $x \notin \bar{y}$. Then $\mathcal{U}$ is an open neighborhood of $x$ in $\mathcal{X}$ and $\operatorname{str}\left(\mathcal{U}^{\prime}\right)=\operatorname{str}_{\leq x_{0}}(\mathcal{X})$ for any smaller open neighborhood $\mathcal{U}^{\prime}$ of $x$.

Corollary 2.12. In the situation of Proposition 2.9, if $y$ is elementary then all of the fibres $y_{x}$ of $\varphi$ at the points $x \in \operatorname{str}(\mathcal{X})$ are also elementary.

We are now going to associate with each pluri-nodal scheme $\mathcal{X}$ a simplicial set $N(\mathcal{X})$.

Recall that a simplicial set is an object of the category $\Delta^{\circ} \mathcal{E} n s$ of contravariant functors from $\Delta$ to the category of sets $\mathscr{E} n s$, where $\Delta$ is the category whose objects are the sets $[n]=\{0,1, \ldots, n\}, n \geq 0$, and morphisms are all nondecreasing maps (see [GaZi, Ch. II]). Recall also that the nerve of a small category $\mathscr{D}$ is the simplicial set $N(\mathscr{D})$ for which $N_{p}(\mathscr{D})$ consists of functors $[p] \rightarrow \mathscr{D}$, where the ordered set $[p]$ considered as a category. For a morphism $\gamma:[q] \rightarrow[p]$, the corresponding map $N_{p}(\mathscr{D}) \rightarrow N_{q}(\mathscr{D})$ takes a functor $[p] \rightarrow \mathscr{D}$ to its composition with $\gamma$.

Proposition 2.13. There is a functor $\mathcal{X} \mapsto N(\mathcal{X})$, unique up to a unique isomorphism, from the category of pluri-nodal schemes with pluri-nodal morphisms between them to the category of simplicial sets $\Delta^{\circ} \mathcal{E} n s$ such that

(a) if $\mathcal{X}$ is strictly pluri-nodal, then $N(\mathcal{X})$ is the nerve of the partially ordered set $\operatorname{str}(\mathcal{X})$;

(b) for a surjective étale morphism $\mathcal{X}^{\prime} \rightarrow \mathcal{X}$ one has $\operatorname{Coker}\left(N\left(\mathcal{X}^{\prime} \times X\right.\right.$ $\left.\left.\mathcal{X}^{\prime}\right) \stackrel{\rightarrow}{\rightarrow} N\left(\mathcal{X}^{\prime}\right)\right) \stackrel{\sim}{\rightarrow} N(\mathcal{X})$.

Proof. We fix for each pluri-nodal scheme $\mathcal{X}$ a surjective étale morphism $X^{\prime} \rightarrow X$ with strictly pluri-nodal $X^{\prime}$ so that, if $X$ is strictly pluri-nodal, then $X^{\prime}=\mathcal{X}$, and define $N(\mathcal{X})$ as the cokernel of the pair of morphisms 
$N\left(\operatorname{str}\left(\mathcal{X}^{\prime \prime}\right)\right) \stackrel{\rightarrow}{\rightarrow} N\left(\operatorname{str}\left(\mathcal{X}^{\prime}\right)\right)$, where $\mathcal{X}^{\prime \prime}=\mathcal{X}^{\prime} \times X_{\mathcal{X}} \mathcal{X}^{\prime}$. To see that we get a functor, it suffices to verify that the condition (b) is satisfied when $\mathcal{X}$ and $X^{\prime}$ are strictly pluri-nodal. In this case the required fact easily follows from Lemma 2.10.

Recall also that the geometric realization functor is a functor $\mid$ ? $\mid$ : $\Delta^{\circ} \mathcal{E} n s \rightarrow \mathcal{K} e$ to the category of Kelley spaces $\mathcal{K} e$. (Recall that a Kelley space is a Hausdorff topological space $X$ possessing the property that a subset of $X$ is closed whenever its intersection with each compact subset of $X$ is closed.) The functor $\mid$ ? $\mid$ commutes with direct limits and extends the functor $\Sigma: \Delta \rightarrow \mathcal{K} e$ that takes the standard $n$-simplex $\Delta[n]$ to $\Sigma^{n}=\left\{\left(u_{0}, \ldots, u_{n}\right) \in[0,1]^{n+1} \mid u_{0}+\cdots+u_{n}=1\right\}$ (see [GaZi, Ch. III]). If $C \in \mathrm{Ob}\left(\Delta^{\circ} \mathcal{E} n s\right)$, then $|C|$ is the direct limit of the functor $(\Delta[n] \rightarrow C) \mapsto \Sigma^{n}$ from the category $\Delta / C$ to $\mathcal{K} e$. (For a category $\mathscr{D}$ and a functor $C \in \mathscr{D}^{\circ} \mathcal{E} n s, \mathscr{D} / C$ denotes the category of morphisms from representable functors to $C$.) A subset of $|C|$ which is the image of the interior $\Sigma^{n}$ of some $\Sigma^{n}$ in the above direct limit is called a (simplicial) cell of $|C|$. It follows from [GaZi, Ch. III, §1.8-1.9] that $C$ is a Hausdorff locally contractible space. If $C$ is locally finite (as, for example, $N(\mathcal{X})$ ), then $|C|$ is locally compact. For such $C$, any subset of $|C|$ which is a union of cells is locally contractible. Notice that for an elementary strictly pluri-nodal scheme $\mathcal{X}$ the space $|N(\mathcal{X})|$ is contractible.

Remark 2.14. (i) The canonical map $N(\mathcal{X}) \rightarrow N(\operatorname{str}(\mathcal{X}))$ is not an isomorphism in general. An example is the nodal curve defined by the equation $y^{2}=x^{2}(x+1)$.

(ii) Let $k$ be a non-Archimedean field. What is done in this section is applied to the closed fibre $\mathfrak{X}_{s}$ of a pluri-nodal scheme $\mathfrak{X}$ over $k^{\circ}$. One can, in fact, introduce in the similar way a stratification on the generic fibre $\mathfrak{X}_{\eta}$ of $\mathfrak{X}$ for which the properties of Proposition 2.1 are also true, and in the case, when $\mathfrak{X}$ is strictly pluri-nodal, one can define a map of partially ordered sets from the set of strata of $\mathfrak{X}_{s}$ to the set of strata of $\mathfrak{X}_{\eta}$.

\section{A polysimplicial set associated with a poly-stable scheme}

In this section we introduce a category $\boldsymbol{\Lambda}$, which gives rise to a category of polysimplicial sets $\boldsymbol{\Lambda}^{\circ} \mathcal{E} n s$, and construct a commutative diagram of functors

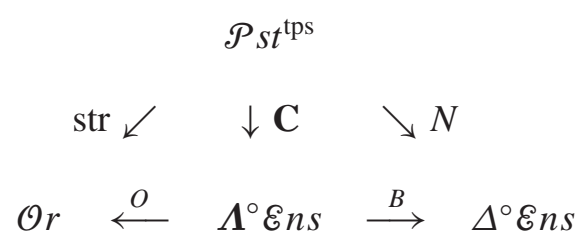

where $\mathcal{O} r$ is the category of partially ordered sets. We also show that the functor $B$ preserves geometric realizations, and introduce a category $\Lambda$ with 
the same family of objects as $\Lambda$ but with smaller sets of morphisms which is enough for constructing a functor $C: \mathcal{P} s t^{\mathrm{sm}} \rightarrow \Lambda^{\circ} \mathcal{E} n s$.

For a tuple $\mathbf{n}=\left(n_{0}, \ldots, n_{p}\right)$ with either $p=n_{0}=0$ or $p \geq 0$ and $n_{i} \geq 1$ for all $0 \leq i \leq p$, let $[\mathbf{n}]$ denote the set $\left[n_{0}\right] \times \cdots \times\left[n_{p}\right]$. (The number $p$ for $[\mathbf{n}]$ as above will be denoted by $w(\mathbf{n})$.) Objects of the category $\boldsymbol{\Lambda}$ are the sets [n] for the tuples $\mathbf{n}$ as above, and the set of morphisms $\operatorname{Hom}([\mathbf{m}],[\mathbf{n}])$ is the set of the maps $[\mathbf{m}] \rightarrow[\mathbf{n}]$ associated with triples $(J, f, \alpha)$ as follows. Let $q=w(\mathbf{m})$. Then $J$ is a subset of $[q]$, assumed to be empty if $[\mathbf{m}]=[0], f$ is an injective map $J \rightarrow[p]$, and $\alpha=\left\{\alpha_{l}\right\}_{0 \leq l<p}$, where $\alpha_{l}$ is an injective map $\left[m_{f^{-1}(l)}\right] \rightarrow\left[n_{l}\right]$ for $l \in \operatorname{Im}(f)$, and $\alpha_{l}$ is a map [0] $\rightarrow\left[n_{l}\right]$ for $l \notin \operatorname{Im}(f)$. The map $\gamma:[\mathbf{m}] \rightarrow[\mathbf{n}]$ associated with the triple $(J, f, \alpha)$ takes an element $\mathbf{j}=\left(j_{0}, \ldots, j_{q}\right) \in[\mathbf{m}]$ to the element $\mathbf{i}=\left(i_{0}, \ldots, i_{p}\right) \in[\mathbf{n}]$ with $i_{l}=\alpha_{l}\left(j_{f^{-1}(l)}\right)$ for $l \in \operatorname{Im}(f)$, and $i_{l}=\alpha_{l}(0)$ for $l \notin \operatorname{Im}(f)$. (Note that different triples give rise to different maps.) It follows from the definition that the composition of two maps of the above form has of the same form, and so $\Lambda$ is really a category. For a subset $J \subset[q]$, assumed to be empty if [m] $=[0]$, let $\mathbf{m}_{J}$ denote the tuple $\left(m_{j_{0}}, \ldots, m_{j_{t}}\right)$, if $J=\left\{j_{0}, \ldots, j_{t}\right\}$ is non-empty and $j_{0}<\cdots<j_{t}$, and the zero tuple 0 , otherwise. It follows that the above morphism $\gamma:[\mathbf{m}] \rightarrow[\mathbf{n}]$ is a composition of the projection $\pi_{I}:[\mathbf{m}] \rightarrow\left[\mathbf{m}_{J}\right]$ (which is a morphism in $\boldsymbol{\Lambda}$ ) with an injective morphism $\sigma:\left[\mathbf{m}_{J}\right] \rightarrow$ [n]. If $\gamma$ is surjective, then $\sigma$ is an isomorphism and, therefore, $\gamma$ has a right inverse, i.e., there exists a morphism $\beta:[\mathbf{n}] \rightarrow[\mathbf{m}]$ with $\gamma \circ \beta=1_{[\mathbf{n}]}$. In particular, a morphism $\gamma:[\mathbf{m}] \rightarrow[\mathbf{n}]$ is an isomorphism if and only if it is bijective. Notice that the automorphism group $\operatorname{Aut}([\mathbf{n}])$ for $\mathbf{n}$ of the form $(n, \ldots, n)$ is the wreath product (see [Hall, §5.9]) of the symmetric group $S_{n+1}$ with the symmetric group $S_{p+1}$, where $p=w(\mathbf{n})$. This easily describes Aut([n]) for an arbitrary $[\mathbf{n}]$.

We now give an alternative description of injective morphisms in $\boldsymbol{\Lambda}$. First of all, any set $[\mathbf{n}] \in \operatorname{Ob}(\boldsymbol{\Lambda})$ is endowed with a metric. Namely, the distance between two elements $\mathbf{i}=\left(i_{0}, \ldots, i_{p}\right) \in[\mathbf{n}]$ and $\mathbf{j}=\left(j_{0}, \ldots, j_{p}\right) \in[\mathbf{n}]$ is the number of distinct coordinates of $\mathbf{i}$ and $\mathbf{j}$. Note that each injective morphism in $\boldsymbol{\Lambda}$ is an isometric map.

Lemma 3.1. Each isometric map $[\mathbf{m}] \rightarrow[\mathbf{n}]$ is an injective morphism in $\boldsymbol{\Lambda}$.

Proof. We may assume that $[\mathbf{m}] \neq[0]$. Let $p=w(\mathbf{n})$ and $q=w(\mathbf{m})$. We have to verify that each isometric map $\gamma:[\mathbf{m}] \rightarrow[\mathbf{n}]$ is associated with a triple of the form $([q], f, \alpha)$.

Case $q=0$. In this case the required fact follows from the simple remark that if $I$ is a subset of [n] with the property that the distance between any two distinct elements of $I$ is equal to 1 , then there exists $0 \leq l \leq p$ such that, for any $0 \leq i \leq p$ with $i \neq l$, the $i$-th coordinates of all of the elements of $I$ are equal.

Case $\mathbf{m}=(1,1)$. In this case the set $[\mathbf{m}]$ consists of four elements. It is easy to describe all of the possible isometric maps $\gamma$, and this description shows the required fact. 
Case $q \geq 1$. Fix $0 \leq k \leq q$, and let $\mathbf{m}^{\prime}=\left(m_{0}, \ldots, m_{k-1}, m_{k+1}, \ldots, m_{q}\right)$. For $\mathbf{j}^{\prime} \in\left[\mathbf{m}^{\prime}\right]$, let $v_{\mathbf{j}^{\prime}}$ be the map $\left[m_{k}\right] \rightarrow[\mathbf{m}]$ associated with the triple ([0], $f, \alpha)$, where $f:[0] \rightarrow[q]$ takes 0 to $k$ and $\alpha$ is defined by $\alpha_{k}=1_{\left[m_{k}\right]}$ and $\alpha_{i}(0)=j_{i}^{\prime}$ for $i \neq k$. We get an isometric map $\gamma_{\mathbf{j}^{\prime}}^{k}=\gamma \circ v_{\mathbf{j}^{\prime}}:\left[m_{k}\right] \rightarrow[\mathbf{n}]$. By the case $q=0, \gamma_{\mathbf{j}^{\prime}}^{k}$ is associated with a triple $\left([0], f_{\mathbf{j}^{\prime}}^{k}, \alpha_{\mathbf{j}^{\prime}}^{k}\right)$, where $f_{\mathbf{j}^{\prime}}^{k}:[0] \rightarrow[p]$ is defined by the number $l^{(k)}=f_{\mathbf{j}^{\prime}}^{k}(0)$, and $\alpha_{\mathbf{j}^{\prime}}^{k}$ is defined by the map $\alpha^{(k)}=\alpha_{\mathbf{j}^{\prime}, l^{(k)}}^{k}:\left[m_{k}\right] \rightarrow\left[n_{l^{(k)}}\right]$ and the numbers $\alpha_{\mathbf{j}^{\prime}, i}^{k}(0)$ with $0 \leq i \leq p$ and $i \neq l^{(k)}$. We claim that the number $l^{(k)}$ and the map $\alpha^{(k)}$ do not depend on $\mathbf{j}^{\prime}$. Indeed, let $l^{\prime \prime}$ and $\alpha^{\prime \prime}$ be the number and the map corresponding to an element $\mathbf{j}^{\prime \prime} \in\left[\mathbf{m}^{\prime}\right]$. To show that $l^{\prime \prime}=l^{\prime}:=l^{(k)}$ and $\alpha^{\prime \prime}=\alpha^{\prime}:=\alpha^{(k)}$, it suffices to assume that the distance between $\mathbf{j}^{\prime}$ and $\mathbf{j}^{\prime \prime}$ in $\left[\mathbf{m}^{\prime}\right]$ is equal to 1 . But if so, the equality $l^{\prime \prime}=l^{\prime}$ follows easily from the case $\mathbf{m}=(1,1)$. Furthermore, for $j \in\left[m_{k}\right]$ the elements $v_{\mathbf{j}^{\prime}}(j)$ and $v_{\mathbf{j}^{\prime \prime}}(j)$ are distinct at only one coordinate $k^{\prime} \neq k$, and therefore the elements $\gamma_{\mathbf{j}^{\prime}}^{k}(j)$ and $\gamma_{\mathbf{j}^{\prime \prime}}^{k}(j)$ are distinct at only one coordinate $l^{\left(k^{\prime}\right)}$. To prove the equality $\alpha^{\prime}(j)=\alpha^{\prime \prime}(j)$, it suffices to verify that $l^{\left(k^{\prime}\right)} \neq l^{(k)}$. But this also easily follows from the case $\mathbf{m}=(1,1)$. Thus, we get an injective map $[q] \rightarrow[p]: k \mapsto l^{(k)}$ and, for each $k \in[q]$, an injective map $\alpha^{(k)}:\left[m_{k}\right] \rightarrow\left[n_{l^{(k)}}\right]$ such that for every $\mathbf{j} \in[\mathbf{m}]$ the $l^{(k)}$ 'th coordinate of $\gamma(\mathbf{j})$ is $\alpha^{(k)}\left(j_{k}\right)$ and all of the coordinates of $\gamma(\mathbf{j})$ outside the places $l^{(0)}, \ldots, l^{(q)}$ do not depend on $\mathbf{j}$. This gives the required fact.

The category of polysimplicial sets is the category $\boldsymbol{\Lambda}^{\circ} \mathcal{E} n s$. The standard n-polysimplex $\boldsymbol{\Lambda}[\mathbf{n}]$ is the object representable by [n]. If $C \in \mathrm{Ob}\left(\boldsymbol{\Lambda}^{\circ} \mathcal{E} n s\right)$, the image of [n] under $C$ is denoted by $C_{\mathbf{n}}$ (the set of $\mathbf{n}$-polysimplices of $C$ ) and, for $f:[\mathbf{m}] \rightarrow[\mathbf{n}]$, the corresponding map $C_{\mathbf{n}} \rightarrow C_{\mathbf{m}}$ will be denoted by $C(f)$. One evidently has $\operatorname{Hom}(\boldsymbol{\Lambda}[\mathbf{n}], C) \stackrel{\sim}{\rightarrow} C_{\mathbf{n}}$ and, in particular, there is a canonical bijection between the set $\bigsqcup C_{\mathrm{n}}$ of all polysimplices of $C$ and the set $\operatorname{Ob}(\boldsymbol{\Lambda} / C)$ of objects of the category $\boldsymbol{\Lambda} / C$. The $\mathbf{n}$-polysimplex of $\boldsymbol{\Lambda}[\mathbf{n}]$ corresponding to the identity morphism $\Lambda[\mathbf{n}] \rightarrow \Lambda[\mathbf{n}]$ is called fundamental and is denoted by $e_{\mathbf{n}}$. A polysimplex $x \in C_{\mathbf{n}}$ is said to be degenerate if there exists a non-isomorphic surjective morphism $f:[\mathbf{n}] \rightarrow[\mathbf{m}]$ with $x \in \operatorname{Im}(C(f))$. Let $C_{\mathbf{n}}^{\text {nd }}$ denote the subset of nondegenerate polysimplices of $C_{\mathbf{n}}$.

Lemma 3.2 (Eilenberg-Zilber Lemma). Let $x \in C_{\mathbf{n}}$ and $p=w(\mathbf{n})$. Then

(i) there exist a unique pair $(I, y)$, consisting of a subset $I \subset[p]$ and a polysimplex $y \in C_{\mathbf{n}_{I}}^{\mathrm{nd}}$, such that $x=C\left(\pi_{I}\right) y$;

(ii) given a surjective morphism $f:[\mathbf{n}] \rightarrow[\mathbf{m}]$ and $z \in C_{\mathbf{m}}$ with $x=C(f) z$, there exists a unique surjective morphism $g:[\mathbf{m}] \rightarrow\left[\mathbf{n}_{I}\right]$ with $z=C(g) y$ and $g \circ f=\pi_{I}$.

Proof. (i) That such a pair $(I, y)$ exists is trivial. Let $\left(I^{\prime} \subset[p], y^{\prime} \in C_{\mathbf{n}_{I^{\prime}}}\right)$ be another pair with the same property. Take an arbitrary right inverse to $\pi_{I}$, $\sigma:\left[\mathbf{n}_{I}\right] \rightarrow[\mathbf{n}]$. Then $y=C\left(\pi_{I^{\prime}} \circ \sigma\right) y^{\prime}$ and, since $y$ is nondegenerate, it 
follows that $\pi_{I^{\prime}} \circ \sigma:\left[\mathbf{n}_{I}\right] \rightarrow\left[\mathbf{n}_{I^{\prime}}\right]$ is injective. This implies that $I \subset I^{\prime}$. For the same reason, $I^{\prime} \subset I$ and, therefore, $I=I^{\prime}$. It follows also that $\pi_{I^{\prime}} \circ \sigma=1_{\mathbf{n}_{I}}$ and, therefore, $y=y^{\prime}$.

(ii) The morphism $f$ can be represented in a unique way as a composition of the projection $\pi_{J}:[\mathbf{n}] \rightarrow\left[\mathbf{n}_{J}\right]$ with an isomorphism $h:\left[\mathbf{n}_{J}\right] \stackrel{\sim}{\rightarrow}[\mathbf{m}]$. By (i), there exist a unique subset $J^{\prime} \subset J$ and a unique polysimplex $y^{\prime} \in C_{\mathbf{n}_{J^{\prime}}}^{\text {nd }}$ with $C(h) z=C\left(\pi^{\prime}\right) y^{\prime}$, where $\pi^{\prime}$ is the canonical projection $\left[\mathbf{n}_{J}\right] \rightarrow\left[\mathbf{n}_{J^{\prime}}\right]$. We get $C\left(\pi_{J^{\prime}}\right) y^{\prime}=C\left(\pi_{J}\right)(C(h) z)=C(f) z=x$ and, by (i) again, $J^{\prime}=I$ and $y^{\prime}=y$. If $\pi$ is the canonical projection $\left[\mathbf{n}_{J}\right] \rightarrow\left[\mathbf{n}_{I}\right]$, then for $g=\pi \circ h^{-1}$ one has $C(g) y=z$ and $g \circ f=\pi_{I}$. The unicity of $g$ follows from (i) applied to the polysimplex $C(h) z \in C_{\mathbf{n}_{J}}$.

Lemma 3.2 implies that there is a bijection $\amalg C_{\mathbf{n}_{I}}^{\text {nd }} \stackrel{\sim}{\rightarrow} C_{\mathbf{n}}$, where $I$ runs through subsets of $[p]$ and each map $C_{\mathbf{n}_{I}}^{\text {nd }} \rightarrow C_{\mathbf{n}}$ is $C\left(\pi_{I}\right)$. It also implies that a morphism of polysimplicial sets $E \rightarrow C$ is injective (resp. bijective) if and only if, for every $\mathbf{n}$, the image of $E_{\mathbf{n}}^{\text {nd }}$ in $C_{\mathbf{n}}$ is contained in $C_{\mathbf{n}}^{\text {nd }}$ and the induced map $E_{\mathbf{n}}^{\text {nd }} \rightarrow C_{\mathbf{n}}^{\text {nd }}$ is injective (resp. bijective).

For a polysimplicial set $C$, let $O(C)$ denote the partially ordered set associated with the category $\Lambda / C$ (see [GaZi, Ch. II, §5.1]). Namely, it is the partially ordered set associated with the set $\mathrm{Ob}(\Lambda / C)$ of polysimplices of $C$ endowed with the following partial preorder structure: for $x \in C_{\mathbf{n}}$ and $y \in C_{\mathbf{m}}, x \leq y$ if there exists a morphism $f:[\mathbf{n}] \rightarrow[\mathbf{m}]$ with $x=C(f) y$. As a set, $O(C)$ is the set of equivalence classes of polysimplices of $C$ with respect to the following equivalence relation: for $x$ and $y$ as above, $x \sim y$ if there exist morphisms $f:[\mathbf{n}] \rightarrow[\mathbf{m}]$ and $g:[\mathbf{m}] \rightarrow[\mathbf{n}]$ with $x=C(f) y$ and $y=C(g) x$. Lemma 3.2 implies that $O(C)$ coincides with the set of equivalence classes of nondegenerate polysimplices. For example, for $\mathbf{n}=\left(n_{0}, \ldots, n_{p}\right), O(\boldsymbol{\Lambda}[\mathbf{n}])$ is canonically isomorphic to the partially ordered (by inclusion) set of all subsets $A \subset[\mathbf{n}]$ with the property $A=\pi_{0}(A) \times \cdots \times \pi_{p}(A)$, where $\pi_{i}$ is the projection $[\mathbf{n}] \rightarrow\left[n_{i}\right]$. The correspondence $C \mapsto O(C)$ is a functor from $\Lambda^{\circ} \mathcal{E} n s$ to the category $\mathcal{O} r$ of partially ordered sets.

Lemma 3.3. The functor $\Lambda^{\circ} \mathcal{E} n s \rightarrow \mathcal{O} r: C \mapsto O(C)$ commutes with direct limits.

Proof. It is evident that the functor commutes with coproducts, and so it suffices to verify that if $\operatorname{Coker}\left(C^{\prime \prime} \stackrel{\rightarrow}{\rightarrow} C^{\prime}\right) \stackrel{\sim}{\rightarrow} C$ then $\operatorname{Coker}\left(O\left(C^{\prime \prime}\right) \stackrel{\rightarrow}{\rightarrow}\right.$ $\left.O\left(C^{\prime}\right)\right) \stackrel{\sim}{\rightarrow} O(C)$. Let $x^{\prime} \in C_{\mathrm{n}}^{\prime}$ and $y^{\prime} \in C_{\mathrm{m}}^{\prime}$, and let $x$ and $y$ be their images in $C_{\mathbf{n}}$ and $C_{\mathbf{m}}$, respectively. First we have to check that if $x \sim y$, then there exist $\widetilde{x}^{\prime} \approx x^{\prime}$ and $\widetilde{y}^{\prime} \approx y^{\prime}$ with $\widetilde{x}^{\prime} \sim \tilde{y}^{\prime}$, where $\approx$ denotes the equivalence relation on the set of polysimplices of $C^{\prime}$ induced by the pair of morphisms $C^{\prime \prime} \rightarrow C^{\prime}$. Take surjective morphisms $f:[\mathbf{n}] \rightarrow[\widetilde{\mathbf{n}}]$ and $g:[\mathbf{m}] \rightarrow[\widetilde{\mathbf{m}}]$ for which there exist nondegenerate $\widetilde{x} \in C_{\widetilde{\mathbf{n}}}$ and $\tilde{y} \in C_{\widetilde{\mathbf{m}}}$ with $x=C(f) \widetilde{x}$ and $y=C(g) \tilde{y}$. By Lemma 3.2, there exists an isomorphism $\sigma:[\tilde{\mathbf{m}}] \stackrel{\sim}{\rightarrow}[\widetilde{\mathbf{n}}]$ with 
$\tilde{y}=C(\sigma) \tilde{x}$. Let $x_{1}^{\prime}$ be a preimage of $\tilde{x}$. Then $C^{\prime}(f) x_{1}^{\prime} \approx x^{\prime}, C^{\prime}(\sigma g) x_{1}^{\prime} \approx y^{\prime}$ and $C^{\prime}(f) x_{1}^{\prime} \sim x_{1}^{\prime} \sim C^{\prime}(\sigma g) x_{1}^{\prime}$. We also have to check that if $x \leq y$, then there exist $\widetilde{x}^{\prime} \approx x^{\prime}$ and $\widetilde{y}^{\prime} \approx y^{\prime}$ with $\widetilde{x}^{\prime} \leq \widetilde{y}^{\prime}$. For this we take a morphism $f:[\mathbf{n}] \rightarrow[\mathbf{m}]$ with $x=C(f) y$, and we get $C^{\prime}(f) y^{\prime} \approx x^{\prime}$ and $C^{\prime}(f) y^{\prime} \leq y^{\prime}$.

By [GaZi, Ch. II, 1.3], the functor $O$ is left adjoint to the functor $\mathcal{O} r \rightarrow$ $\Lambda^{\circ} \mathcal{E} n s$ that associates with a partially ordered set $E$ the simplicial set whose sets of $\mathbf{n}$-polysimplices are the sets of maps of partially ordered sets $O(\Lambda[\mathbf{n}]) \rightarrow E$.

Let $d \geq 0$. The $d$-skeleton $\mathrm{Sk}^{d}(C)$ of a polysimplicial set $C$ is the polysimplicial subset $C^{\prime} \subset C$ such that $C_{\mathbf{n}}^{\prime}$ is formed by all polysimplices degenerated from $\mathbf{m}$-polysimplices with $|\mathbf{m}| \leq d$. (For $\mathbf{m}=\left(m_{0}, \ldots, m_{q}\right)$, $|\mathbf{m}|=m_{0}+\cdots+m_{q}$.) We say that $C$ is of dimension $\leq d$ if $C=\operatorname{Sk}^{d}(C)$. For example, the standard n-polysimplex $\boldsymbol{\Lambda}[\mathbf{n}]$ is of dimension $|\mathbf{n}|$, and each nondegenerate $\mathbf{n}^{\prime}$-polysimplex with $\left|\mathbf{n}^{\prime}\right| \geq|\mathbf{n}|$ is equivalent to the fundamental $\mathbf{n}$-polysimplex of $\boldsymbol{\Lambda}[\mathbf{n}]$. In particular, each polysimplex of $\boldsymbol{\Lambda}[\mathbf{n}]$, non-equivalent to the fundamental one, is contained in the $(|\mathbf{n}|-1)$ skeleton $\mathrm{Sk}^{|\mathbf{n}|-1}(\boldsymbol{\Lambda}[\mathbf{n}])$. The latter is called the boundary of $\boldsymbol{\Lambda}[\mathbf{n}]$ and is denoted by $\dot{\Lambda}[\mathbf{n}]$. Furthermore, for a polysimplex $x \in C_{\mathbf{n}}$, let $G_{x}$ denote the stabilizer of $x$ in $\operatorname{Aut}([\mathbf{n}])$. The morphism $\boldsymbol{\Lambda}[\mathbf{n}] \rightarrow C$ that corresponds to $x$ goes through a morphism $G_{x} \backslash \boldsymbol{\Lambda}[\mathbf{n}] \rightarrow C$.

Lemma 3.4. Let $A^{d}$ be a set of representatives of the equivalence classes of nondegenerate polysimplices of $C$ of dimension $d$. Then the following diagram is cocartesian

$$
\begin{aligned}
& \bigsqcup_{x \in A^{d}} G_{x} \backslash \dot{\Lambda}\left[\mathbf{n}_{x}\right] \longrightarrow \operatorname{Sk}^{d-1}(C) \\
& \downarrow \\
& \bigsqcup_{x \in A^{d}} G_{x} \backslash \Lambda\left[\mathbf{n}_{x}\right] \longrightarrow \mathrm{Sk}^{d}(C)
\end{aligned}
$$

Lemma 3.5. Let a finite group $G$ act on a polysimplicial set $C$. Then for any $\mathbf{n}$ one has $(G \backslash C)_{\mathbf{n}}^{\mathrm{nd}}=G \backslash C_{\mathbf{n}}^{\mathrm{nd}}$.

Proof. Let $\varphi$ denote the canonical surjective morphism $C \rightarrow C^{\prime}=G \backslash C$. For $x \in C_{\mathbf{n}}^{\text {nd }}$, let $I$ be the subset of $[p], p=w(\mathbf{n})$, for which there exists a nondegenerate $y^{\prime} \in C_{\mathbf{n}_{I}}^{\prime}$ with $\varphi_{\mathbf{n}}(x)=C^{\prime}\left(\pi_{I}\right) y^{\prime}$. If $\sigma$ is a right inverse of $\pi_{I}$, then for $y=C(\sigma) x$ one has $\varphi_{\mathbf{n}_{I}}(y)=y^{\prime}$ and $\varphi_{\mathbf{n}}\left(C\left(\pi_{I}\right) y\right)=\varphi_{\mathbf{n}}(x)$ and, therefore, there exists an element $g \in G$ with $x=g C\left(\pi_{I}\right) y=C\left(\pi_{I}\right)(g y)$. This implies that $I=[p]$ and $x=g y$, i.e., $\varphi_{\mathbf{n}}(x)$ is nondegenerate. It follows that $G \backslash C_{\mathbf{n}}^{\text {nd }} \subset(G \backslash C)_{\mathbf{n}}^{\text {nd }}$. The converse inclusion is evident.

Proof of Lemma 3.4. Let $C^{\prime}$ be the cocartesian product, and let $D$ and $D^{\prime}$ denote the polysimplicial sets at the north-west and the south-west of the diagram, respectively. If $|\mathbf{m}|<d$, then $D_{\mathbf{m}} \stackrel{\sim}{\rightarrow} D_{\mathbf{m}}^{\prime}$ and $\mathrm{Sk}^{d-1}(C)_{\mathbf{m}} \stackrel{\sim}{\rightarrow} \operatorname{Sk}^{d}(C)_{\mathbf{m}}$ 
and, therefore, $C_{\mathbf{m}}^{\prime} \stackrel{\sim}{\rightarrow} \operatorname{Sk}^{d}(C)_{\mathbf{m}}$. Furthermore, Lemma 3.5 implies that the lower arrow of the diagram induces a bijection between the set $X$ of nondegenerate polysimplices of $D^{\prime}$ of dimension $d$ and the set $Y$ of nondegenerate polysimplices of $C$ of dimension $d$. If now $|\mathbf{m}| \geq d$ and $q=w(\mathbf{m})$, then the complement of $D_{\mathbf{m}}$ in $D_{\mathbf{m}}^{\prime}$ (resp. of $\mathrm{Sk}^{d-1}(C)_{\mathbf{m}}$ in $\left.\mathrm{Sk}^{d}(C)_{\mathbf{m}}\right)$ consists of the polysimplices degenerated from $X$ (resp. $Y$ ). By the Eilenberg-Zilber Lemma 3.2, the latter set corresponds bijectively to the set of pairs $(J, x)$ (resp. $(J, y)$ ), where $J$ is a subset of $[q]$ with $\left|\mathbf{m}_{J}\right|=d$ and $x \in D_{\mathbf{m}_{J}}^{\text {nd }} \subset X$ (resp. $y \in C_{\mathbf{m}_{J}}^{\text {nd }} \subset Y$ ). It follows that $C_{\mathbf{m}}^{\prime} \stackrel{\sim}{\rightarrow} \operatorname{Sk}^{d}(C)_{\mathbf{m}}$.

The category $\boldsymbol{\Lambda}$ is a symmetric strict monoidal category (see [Mac, Ch. VII]) with respect to the multiplication bifunctor $\boldsymbol{\Lambda} \times \boldsymbol{\Lambda} \stackrel{\square}{\rightarrow} \boldsymbol{\Lambda}$ defined by $[0] \square[\mathbf{n}]=[\mathbf{n}]\left[[0]=[\mathbf{n}]\right.$ and, if $\mathbf{n}^{\prime}=\left(n_{0}^{\prime}, \ldots, n_{p^{\prime}}^{\prime}\right)$ and $\mathbf{n}^{\prime \prime}=$ $\left(n_{0}^{\prime \prime}, \ldots, n_{p^{\prime \prime}}^{\prime \prime}\right)$ are different from 0 , then $\left[\mathbf{n}^{\prime}\right]\left[\left[\mathbf{n}^{\prime \prime}\right]=[\mathbf{n}]\right.$, where $\mathbf{n}=$ $\left(n_{0}^{\prime}, \ldots, n_{p^{\prime}}^{\prime}, n_{0}^{\prime \prime}, \ldots, n_{p^{\prime \prime}}^{\prime \prime}\right)$. The above structure on $\boldsymbol{\Lambda}$ is naturally extended to a similar structure on the category of polysimplicial sets $\Lambda^{\circ} \& n s$, i.e., there is a bifunctor $\Lambda^{\circ} \mathfrak{E} n s \times \Lambda^{\circ} \mathcal{E} n s \stackrel{\text { ㅁ }}{\longrightarrow} \Lambda^{\circ} \mathcal{E} n s:\left(C^{\prime}, C^{\prime \prime}\right) \mapsto C^{\prime}$ 미 $C^{\prime \prime}$ that commutes with direct limits and is defined by $C^{\prime} a C^{\prime \prime}=\lim \Lambda[\mathbf{n}]$, where the limit is taken over all $\left(\boldsymbol{\Lambda}\left[\mathbf{n}^{\prime}\right] \rightarrow C^{\prime}\right) \in \mathrm{Ob}\left(\boldsymbol{\Lambda} / C^{\prime}\right)$ and $\left(\boldsymbol{\Lambda}\left[\mathbf{n}^{\prime \prime}\right] \rightarrow C^{\prime \prime}\right) \in$ $\mathrm{Ob}\left(\boldsymbol{\Lambda} / C^{\prime \prime}\right)$, and $[\mathbf{n}]=\left[\mathbf{n}^{\prime}\right] \mathrm{b}\left[\mathbf{n}^{\prime \prime}\right]$. We remark that the canonical morphisms from $\left[\mathbf{n}^{\prime}\right]\left[\left[\mathbf{n}^{\prime \prime}\right]\right.$ to $\left[\mathbf{n}^{\prime}\right]$ and $\left[\mathbf{n}^{\prime \prime}\right]$ induce a morphism of functors $\square \rightarrow \times$, where $\times$ is the direct product bifunctor $\Lambda^{\circ} \mathscr{E} n s \times \Lambda^{\circ} \mathscr{E} n s \stackrel{\times}{\rightarrow} \Lambda^{\circ} \mathscr{E} n s:$ $\left(C^{\prime}, C^{\prime \prime}\right) \mapsto C^{\prime} \times C^{\prime \prime}$ (which defines another symmetric strict monoidal category structure on $\left.\boldsymbol{\Lambda}^{\circ} \mathcal{E} n s\right)$. We also remark that the correspondence $\left(\boldsymbol{\Lambda}\left[\mathbf{n}^{\prime}\right] \rightarrow C^{\prime}, \boldsymbol{\Lambda}\left[\mathbf{n}^{\prime \prime}\right] \rightarrow C^{\prime \prime}\right) \mapsto(\boldsymbol{\Lambda}[\mathbf{n}] \rightarrow C)$, where $[\mathbf{n}]=\left[\mathbf{n}^{\prime}\right]\left[\left[\mathbf{n}^{\prime \prime}\right]\right.$, defines a map $\mathrm{Ob}\left(\boldsymbol{\Lambda} / C^{\prime}\right) \times \mathrm{Ob}\left(\boldsymbol{\Lambda} / C^{\prime \prime}\right) \rightarrow \mathrm{Ob}\left(\boldsymbol{\Lambda} / C^{\prime}\right.$ a $\left.C^{\prime \prime}\right)$. For $x^{\prime} \in C_{\mathrm{n}^{\prime}}^{\prime}$ and $x^{\prime \prime} \in C_{\mathbf{n}^{\prime \prime}}^{\prime \prime}$ we denote by $\left\langle x^{\prime}, x^{\prime \prime}\right\rangle$ the corresponding element of $\left(C^{\prime} \square C^{\prime \prime}\right)_{\mathbf{n}}$, $[\mathbf{n}]=\left[\mathbf{n}^{\prime}\right] \square\left[\mathbf{n}^{\prime \prime}\right]$. Finally, for two disjoint subsets $I^{\prime}, I^{\prime \prime} \subset[p]$, where $p=w(\mathbf{n})$, we denote by $\sigma_{I^{\prime}, I^{\prime \prime}}$ the evident isomorphism $\left[\mathbf{n}_{I^{\prime} \cup I^{\prime \prime}}\right] \stackrel{\sim}{\rightarrow}\left[\mathbf{n}_{I^{\prime}}\right] \mathrm{a}\left[\mathbf{n}_{I^{\prime \prime}}\right]$ and by $\pi_{I^{\prime}, I^{\prime \prime}}$ the composition $\sigma_{I^{\prime}, I^{\prime \prime}} \circ \pi_{I^{\prime} \cup I^{\prime \prime}}:[\mathbf{n}] \rightarrow\left[\mathbf{n}_{I^{\prime}}\right]\left[\left[\mathbf{n}_{I^{\prime \prime}}\right]\right.$.

Lemma 3.6. Let $x \in\left(C^{\prime} \text { a } C^{\prime \prime}\right)_{\mathbf{n}}$ and $p=w(\mathbf{n})$. Then there exist a unique tuple $\left(I^{\prime}, I^{\prime \prime}, x^{\prime}, x^{\prime \prime}\right)$, consisting of disjoint subsets $I^{\prime}, I^{\prime \prime} \subset[p]$ and polysimplices $x^{\prime} \in C_{\mathbf{n}_{I^{\prime}}}^{\text {nd }}$ and $x^{\prime \prime} \in C_{\mathbf{n}_{I^{\prime \prime}}}^{\prime \text {,nd }}$, such that $x=\left(C^{\prime}\right.$ 口 $\left.C^{\prime \prime}\right)\left(\pi_{I^{\prime}, I^{\prime \prime}}\right)\left\langle x^{\prime}, x^{\prime \prime}\right\rangle$.

Lemma 3.6 implies that for any pair $x^{\prime} \in C_{\mathbf{n}^{\prime}}^{\prime \text { nd }}$ and $x^{\prime \prime} \in C_{\mathbf{n}^{\prime \prime}}^{\prime \prime n d}$ one has $\left\langle x^{\prime}, x^{\prime \prime}\right\rangle \in\left(C^{\prime} \square C^{\prime \prime}\right)_{\mathbf{n}}^{\text {nd }}$, where $[\mathbf{n}]=\left[\mathbf{n}^{\prime}\right] \square\left[\mathbf{n}^{\prime \prime}\right]$. It also implies that there is a bijection $\amalg C_{\mathbf{n}_{I^{\prime}}}^{\text {nd }} \times C_{\mathbf{n}_{I^{\prime \prime}}}^{\prime \prime \text { nd }} \stackrel{\sim}{\rightarrow}\left(C^{\prime} \text { a } C^{\prime \prime}\right)_{\mathbf{n}}$, where $I^{\prime}$ and $I^{\prime \prime}$ run through disjoint subsets of $[p](p=w(\mathbf{n}))$.

Proof. The morphism $\Lambda[\mathbf{n}] \rightarrow C^{\prime}$ ' $C^{\prime \prime}$ that corresponds to $x$ goes through the canonical morphism $\Lambda\left[\mathbf{m}^{\prime}\right] \square \Lambda\left[\mathbf{m}^{\prime \prime}\right] \rightarrow C^{\prime} \square C^{\prime \prime}$ for some morphisms $\boldsymbol{\Lambda}\left[\mathbf{m}^{\prime}\right] \rightarrow C^{\prime}$ and $\boldsymbol{\Lambda}\left[\mathbf{m}^{\prime \prime}\right] \rightarrow C^{\prime \prime}$. The morphism $\boldsymbol{\Lambda}[\mathbf{n}] \rightarrow \boldsymbol{\Lambda}\left[\mathbf{m}^{\prime}\right] \square \boldsymbol{\Lambda}\left[\mathbf{m}^{\prime \prime}\right]$ is a composition of a projection $\Lambda[\mathbf{n}] \rightarrow \Lambda\left[\mathbf{n}_{J}\right]$ and an injective morphism 
$\boldsymbol{\Lambda}\left[\mathbf{n}_{J}\right] \rightarrow \boldsymbol{\Lambda}\left[\mathbf{m}^{\prime}\right] \square \boldsymbol{\Lambda}\left[\mathbf{m}^{\prime \prime}\right]$. The latter is defined by a partition $J=J^{\prime} \amalg J^{\prime \prime}$ and injective morphisms $\Lambda\left[\mathbf{n}_{J^{\prime}}\right] \rightarrow \Lambda\left[\mathbf{m}^{\prime}\right]$ and $\Lambda\left[\mathbf{n}_{J^{\prime \prime}}\right] \rightarrow \Lambda\left[\mathbf{m}^{\prime \prime}\right]$. If $y^{\prime} \in C_{\mathbf{n}_{J^{\prime}}}^{\prime}$ and $y^{\prime \prime} \in C_{\mathbf{n}_{J^{\prime \prime}}}^{\prime \prime}$ correspond to the induced morphisms $\Lambda\left[\mathbf{n}_{J^{\prime}}\right] \rightarrow C^{\prime}$ and $\boldsymbol{\Lambda}\left[\mathbf{n}_{J^{\prime \prime}}\right] \rightarrow C^{\prime \prime}$, we get $x=\left(C^{\prime} \square C^{\prime \prime}\right)\left(\pi_{J^{\prime}, J^{\prime \prime}}\right)\left\langle y^{\prime}, y^{\prime \prime}\right\rangle$. Let $y^{\prime}=C^{\prime}\left(\pi^{\prime}\right) x^{\prime}$ and $y^{\prime \prime}=C^{\prime \prime}\left(\pi^{\prime \prime}\right) x^{\prime \prime}$, where $x^{\prime} \in C_{\mathbf{n}_{I^{\prime}}}^{\text {nd }}$ and $x^{\prime \prime} \in C_{\mathbf{n}_{I^{\prime \prime}}^{\prime \prime}}^{\text {nd }}$ for some subsets $I^{\prime} \subset J^{\prime}$ and $I^{\prime \prime} \subset J^{\prime \prime}$, and $\pi^{\prime}$ and $\pi^{\prime \prime}$ are the canonical projections $\left[\mathbf{n}_{J^{\prime}}\right] \rightarrow\left[\mathbf{n}_{I^{\prime}}\right]$ and $\left[\mathbf{n}_{J^{\prime \prime}}\right] \rightarrow\left[\mathbf{n}_{I^{\prime \prime}}\right]$. Then we get $x=\left(C^{\prime} \square C^{\prime \prime}\right)\left(\pi_{I^{\prime}, I^{\prime \prime}}\right)\left\langle x^{\prime}, x^{\prime \prime}\right\rangle$. Let now $\varphi^{\prime}$ and $\varphi^{\prime \prime}$ be the canonical morphisms $C^{\prime}{ }^{\prime \prime} C^{\prime \prime} \rightarrow C^{\prime}$ and $C^{\prime}{ }^{\prime \prime} C^{\prime \prime} \rightarrow C^{\prime \prime}$. Then $\varphi_{\mathbf{n}}^{\prime}(x)=C^{\prime}\left(\pi_{I^{\prime}}\right) x^{\prime}$ and $\varphi_{\mathbf{n}}^{\prime \prime}(x)=C^{\prime \prime}\left(\pi_{I^{\prime \prime}}\right) x^{\prime \prime}$. The Eilenberg-Zilber Lemma 3.2 implies that the subsets $I^{\prime}$ and $I^{\prime \prime}$ and the polysimplices $x^{\prime}$ and $x^{\prime \prime}$ are uniquely determined by $x$.

Corollary 3.7. (i) The canonical morphism $C^{\prime} \square C^{\prime \prime} \rightarrow C^{\prime} \times C^{\prime \prime}$ is injective;

(ii) there is a canonical isomorphism of partially ordered sets $O\left(C^{\prime}\right) \times$ $O\left(C^{\prime \prime}\right) \stackrel{\sim}{\rightarrow} O\left(C^{\prime}\right.$ 口C $\left.C^{\prime \prime}\right)$;

(iii) given injective morphisms $E^{\prime} \rightarrow C^{\prime}$ and $E^{\prime \prime} \rightarrow C^{\prime \prime}$, the induced morphism $E^{\prime} \square E^{\prime \prime} \rightarrow C^{\prime}$ ' $C^{\prime \prime}$ is injective.

A polysimplicial set is said to be finite if it has a finite number of nondegenerate polysimplices. It is said to be locally finite if each vertex is contained in a finite number of nondegenerate polysimplices. Furthermore, a polysimplicial set is said to be free if, for each $x \in C_{\mathbf{n}}^{\text {nd }}$, the corresponding morphism $\Lambda[\mathbf{n}] \rightarrow C$ is injective or, equivalently, if each nondegenerate polysimplex has the maximally possible number of vertices (equal to $\left(n_{0}+1\right) \cdot \ldots \cdot\left(n_{p}+1\right)$ for $\mathbf{n}$-polysimplices). It is said to be interiorly free if for each $[\mathbf{n}]$ the action of $\operatorname{Aut}([\mathbf{n}])$ on the set of the nondegenerate n-polysimplices is free. It is clear that a free polysimplicial set is always interiorly free. From Lemma 3.6 it follows that the a-product of two free (resp. interiorly free) polysimplicial sets is free (resp. interiorly free).

We say that a polysimplicial set $C$ is nondegenerate if, for any injective morphism $f:[\mathbf{m}] \rightarrow[\mathbf{n}]$, the map $C(f)$ takes nondegenerate polysimplices to nondegenerate ones. Furthermore, a morphism between polysimplicial sets is said to be nondegenerate if it takes nondegenerate polysimplices to nondegenerate ones. The category of nondegenerate polysimplicial sets with nondegenerate morphisms between them can be described as follows. Let $\Lambda$ be the category with the same family of objects as $\boldsymbol{\Lambda}$ but with only injective morphisms between them. The restriction functor $\Lambda^{\circ} \mathcal{E} n s \rightarrow \Lambda^{\circ} \mathcal{E} n s$ has a left adjoint functor $\Lambda^{\circ} \mathscr{E} n s \rightarrow \Lambda^{\circ} \mathscr{E} n s: C \mapsto \mathbf{C}$ which extends the functor $\Lambda \rightarrow \Lambda \stackrel{h^{\Lambda}}{\rightarrow} \Lambda^{\circ} \mathcal{E} n s$ to the one that commutes with direct limits. One has $\mathbf{C}_{0}=C_{0}$ and, for $[\mathbf{n}] \neq[0], \mathbf{C}_{\mathbf{n}}$ can be identified with the disjoint union $\amalg C_{\mathbf{n}_{I}}$, taken over all subset $I \subset[p]$. For a morphism $[\mathbf{m}] \rightarrow[\mathbf{n}]$ associated with a triple $(J, f, \alpha)$, the map $\mathbf{C}_{\mathbf{n}} \rightarrow \mathbf{C}_{\mathbf{m}}$ is induced by the maps $C_{\mathbf{n}_{I}} \rightarrow C_{\mathbf{m}_{f^{-1}(I)}}$. The functor $C \mapsto \mathbf{C}$ identifies $\Lambda^{\circ} \mathcal{E} n s$ with the category mentioned at the beginning of this paragraph, and one has $O(C) \stackrel{\sim}{\rightarrow} O(\mathbf{C})$. 
The symmetric strict monoidal category structure on $\boldsymbol{\Lambda}$ induces a similar structure on $\Lambda$, and the latter extends to the category $\Lambda^{\circ} \mathcal{E} n s$ and is compatible with the functor $C \mapsto \mathbf{C}$.

We now construct the geometric realization of a polysimplicial set. For this we consider the functor $\Sigma: \Lambda \rightarrow \mathcal{K} e$ that takes [n] to $\Sigma^{\mathbf{n}}=\Sigma^{n_{0}} \times \cdots \times \Sigma^{n_{p}}=\left\{\left(u_{i l}\right)_{0 \leq i \leq p, 0 \leq l \leq n_{i}} \in[0,1]^{[\mathbf{n}]} \mid u_{i 0}+\cdots+u_{i n_{i}}=1\right\}$, where $[0,1]^{[\mathbf{n}]}$ is the space of all maps $[\mathbf{n}] \rightarrow[0,1]$. Given a morphism $\gamma:[\mathbf{m}] \rightarrow[\mathbf{n}]$ associated with a triple $(J, f, \alpha)$, the map $\Sigma(\gamma)$ takes a point $\mathbf{u}=\left(u_{j k}\right)_{0 \leq j \leq q, 0 \leq k \leq m_{j}}$ to the point $\mathbf{u}^{\prime}=\left(u_{i l}^{\prime}\right)_{0 \leq i \leq p, 0 \leq l \leq n_{i}}$, where (a) if $[\mathbf{m}] \neq[0]$ and $i \notin \operatorname{Im}(f)$, or $[\mathbf{m}]=[0]$, then $u_{i l}^{\prime}=1$ for $l=\alpha_{i}(0)$ and $u_{i l}^{\prime}=0$ for $l \neq \alpha_{i}(0)$, and (b) if $[\mathbf{m}] \neq[0]$ and $i \in \operatorname{Im}(f)$, then $u_{i l}^{\prime}=u_{f^{-1}(i), \alpha_{i}^{-1}(l)}$ for $l \in \operatorname{Im}\left(\alpha_{i}\right)$ and $u_{i l}^{\prime}=0$ for $l \notin \operatorname{Im}\left(\alpha_{i}\right)$. The geometric realization functor $|?|: \Lambda^{\circ} \mathcal{E} n s \rightarrow \mathcal{K} e$ extends the functor $\Sigma$ to the one that commutes with direct limits, i.e., for $C \in \mathrm{Ob}\left(\Lambda^{\circ} \mathcal{E} n s\right),|C|$ is the direct limit of the functor $(\boldsymbol{\Lambda}[\mathbf{n}] \rightarrow C) \mapsto \Sigma^{\mathbf{n}}$ from the category $\boldsymbol{\Lambda} / C$ to $\mathcal{K} e$. We remark that, given an action of a group $G$ on a polysimplicial set $C$, there is a canonical isomorphism $G \backslash|C| \stackrel{\sim}{\rightarrow}|G \backslash C|$, and that for any pair of polysimplicial sets $C^{\prime}$ and $C^{\prime \prime}$ there is a canonical homeomorphism

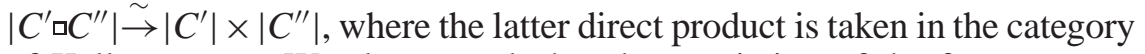
of Kelley spaces. We also remark that the restriction of the functor $\Sigma$ to the category $\Lambda$ gives rise to a geometric realization functor $\mid$ ? $\mid: \Lambda^{\circ} \mathcal{E} n s \rightarrow$ $\mathcal{K} e$ and, for any $C \in \mathrm{Ob}\left(\Lambda^{\circ} \mathcal{E} n s\right)$, there is a canonical homeomorphism $|C| \stackrel{\sim}{\rightarrow}|\mathbf{C}|$.

The interior $\stackrel{\circ}{\Sigma}^{\mathbf{n}}$ of $\Sigma^{\mathbf{n}}$, i.e., the subset of the points that have an open neighborhood homeomorphic to an $|\mathbf{n}|$-dimensional open ball, coincides with $\left.\Sigma^{\mathbf{n}} \cap\right] 0,1\left[{ }^{[\mathbf{n}]}\right.$ if $[\mathbf{n}] \neq[0]$ and with $\Sigma^{\mathbf{n}}$ if $[\mathbf{n}]=[0]$. The boundary $\dot{\Sigma}^{\mathbf{n}}$ of $\Sigma^{\mathbf{n}}$ is the closed subset $\Sigma^{\mathbf{n}} \backslash \stackrel{\circ}{\Sigma}^{\mathbf{n}}$.

Lemma 3.8. The canonical morphism $\dot{\Lambda}[\mathbf{n}] \rightarrow \boldsymbol{\Lambda}[\mathbf{n}]$ induces a homeomorphism $|\dot{\Lambda}[\mathbf{n}]| \stackrel{\sim}{\rightarrow} \dot{\Sigma}^{\mathbf{n}}$.

Proof. Let $\mathcal{A}$ denote the family of subsets $A \subset[\mathbf{n}]$ with the property $A=$ $A_{0} \times \cdots \times A_{p}$, where $A_{i}$ is the image of $A$ under the projection $[\mathbf{n}] \rightarrow\left[n_{i}\right]$. For $A \in \mathcal{A}$, let $\mathbf{n}^{A}$ denote the tuple consisting of the numbers $\# A_{i}-1$ that are greater than zero, and set $|A|=\left|\mathbf{n}^{A}\right|$. There is the evident injective morphism $i_{A}:\left[\mathbf{n}^{A}\right] \rightarrow[\mathbf{n}]$, and the morphisms $i_{A}$ for all $A \in \mathcal{A}$ form a representative set of the equivalence classes of nondegenerate polysimplices of $\Lambda[\mathbf{n}]$. For $B \in \mathcal{A}$ with $B \subset A$, there is a canonical injective morphism $\left[\mathbf{n}^{B}\right] \rightarrow\left[\mathbf{n}^{A}\right]$ whose composition with $i_{A}$ coincides with $i_{B}$. Each $B \in \mathcal{A}$ with $|B|=$ $|\mathbf{n}|-2$ is contained in exactly two subsets $B_{0}, B_{1} \in \mathcal{A}$ with $\left|B_{0}\right|=\left|B_{1}\right|=$ $|\mathbf{n}|-1$. Let $i_{B}^{0}$ and $i_{B}^{1}$ denote the canonical morphisms $\left[\mathbf{n}^{B}\right] \rightarrow\left[\mathbf{n}^{B_{0}}\right]$ and $\left[\mathbf{n}^{B}\right] \rightarrow\left[\mathbf{n}^{B_{1}}\right]$, respectively. We claim that there is a canonical isomorphism of polysimplicial sets

$$
\operatorname{Coker}\left(\coprod \Lambda\left[\mathbf{n}^{B}\right] \stackrel{\rightarrow}{\rightarrow} \coprod \Lambda\left[\mathbf{n}^{A}\right]\right) \stackrel{\sim}{\rightarrow} \dot{\Lambda}[\mathrm{n}],
$$


where the first (resp. second) coproduct is taken over all $B \in \mathcal{A}$ (resp. $A \in \mathcal{A}$ ) with $|B|=|\mathbf{n}|-2$ (resp. $|A|=|\mathbf{n}|-1$ ), and the upper (resp. lower) morphism is induced by the morphisms $i_{B}^{0}$ (resp. $i_{B}^{1}$ ). Indeed, let $D$ denote the cokernel. Since all of the morphisms $\Lambda\left[\mathbf{n}^{A}\right] \rightarrow \dot{\Lambda}[\mathbf{n}]$ are injective, it suffices to check that $D_{\mathbf{m}}^{\text {nd }} \stackrel{\sim}{\rightarrow} \dot{\Lambda}[\mathbf{n}]_{\mathbf{m}}^{\text {nd }}$ for all $|\mathbf{m}|$ with $|\mathbf{m}| \leq|\mathbf{n}|-1$. The surjectivity is evident, and the injectivity for $\mathbf{m}$ with $|\mathbf{m}|=|\mathbf{n}|-1$ directly follows from the construction. Assume that there are two subset $A, A^{\prime} \in \mathcal{A}$ with $|A|=\left|A^{\prime}\right|=|\mathbf{n}|-1$ such that for some $\mathbf{m}$ with $|\mathbf{m}| \leq|\mathbf{n}|-2$ the images of two polysimplices from $\Lambda\left[\mathbf{n}^{A}\right]_{\mathbf{m}}^{\text {nd }}$ and $\boldsymbol{\Lambda}\left[\mathbf{n}^{A^{\prime}}\right]_{\mathbf{m}}^{\text {nd }}$ in $\boldsymbol{\Lambda}[\mathbf{n}]_{\mathbf{m}}$ coincide. This means that there is a subset $C \in \mathcal{A}$ with $C \subset A \cap A^{\prime}$ and $[\mathbf{m}] \stackrel{\sim}{\rightarrow}\left[\mathbf{n}^{C}\right]$, and our claim follows from the simple observation that there exists a sequence of subsets $A_{1}=A, A_{2}, \ldots, A_{l}=A^{\prime}$ with $\left|A_{i}\right|=|\mathbf{n}|-1$ and $C \subset A_{i} \cap A_{i+1}$. The statement of the lemma now follows easily from the fact that the geometric realization functor commutes with cokernels.

Lemmas 3.4 and 3.8 imply that an injective morphism of polysimplicial sets $C^{\prime} \rightarrow C$ induces a homeomorphism of $C^{\prime}$ with a closed subset of $|C|$. Furthermore, a subset of $|C|$, which is the image of the interior $\stackrel{\circ}{\Sigma}^{\mathbf{n}}$ with respect to the map $\Sigma^{\mathbf{n}} \rightarrow|C|$ that corresponds to a polysimplex $x \in C_{\mathbf{n}}$, is called a (polysimplicial) cell of $|C|$. It is clear that for a given cell such a polysimplex $x$ can be chosen to be nondegenerate. In this case Lemmas 3.4 and 3.8 imply that the above map identifies $G_{x} \backslash \stackrel{\circ}{\Sigma}^{\mathbf{n}}$ with the cell. In particular, if $C$ is interiorly free, $\Sigma^{\mathbf{n}}$ is identified with its image in $|C|$. (If $C$ is free, $\Sigma^{\mathbf{n}}$ is identified with its image in $|C|$.) It follows also that $|C|$ is a disjoint union of cells, the closure of a cell is a union of cells, and the image of a cell of $|C|$ under the map induced by a morphism $C \rightarrow C^{\prime}$ is a cell of $\left|C^{\prime}\right|$. Let $O(|C|)$ denote the set of cells of $|C|$ endowed with the partial ordering such that $A \leq B$ if $A \subset \bar{B}$.

Corollary 3.9. For each polysimplicial set $C$, there is a functorial isomorphism of partially ordered sets $O(C) \stackrel{\sim}{\rightarrow} O(|C|)$.

Let $B$ be the functor $\Lambda^{\circ} \mathcal{E} n s \rightarrow \Delta^{\circ} \mathcal{E} n s$ which extends the functor $\boldsymbol{\Lambda} \rightarrow \Delta^{\circ} \mathcal{E}_{n}:[\mathbf{n}] \mapsto N(O(\boldsymbol{\Lambda}[\mathbf{n}]))$ to the one that commutes with direct limits (the barycentric subdivision functor). Notice that, if in the definition of $B$ we use the category $\Lambda$ instead of $\Lambda$, we get a functor $\Lambda^{\circ} \mathcal{E} n s \rightarrow \Delta^{\circ} \mathcal{E} n s$ compatible with $B$ with respect to the functor $C \mapsto \mathbf{C}$.

Lemma 3.10. For each $C \in \mathrm{Ob}\left(\boldsymbol{\Lambda}^{\circ} \mathcal{E} n s\right)$ there is a functorial homeomorphism $|B C| \stackrel{\sim}{\rightarrow}|C|$.

Proof. Since all of the functors considered commute with direct limits, it suffices to construct functorial homeomorphisms $|B \Lambda[\mathbf{n}]| \stackrel{\sim}{\rightarrow} \Sigma^{\mathbf{n}}$. For $\mathbf{n}=\left(n_{0}, \ldots, n_{p}\right)$, let $z\left(\Sigma^{\mathbf{n}}\right)$ denote the point of $\Sigma^{\mathbf{n}}$ with the coordinates $u_{i l}=\frac{1}{n_{i}+1}$ for all $0 \leq i \leq p$ and $0 \leq l \leq n_{i}$. We remark that, given an isomorphism $[\mathbf{m}] \stackrel{\sim}{\rightarrow}[\mathbf{n}]$, the image of $z\left(\Sigma^{\mathbf{m}}\right)$ in $\Sigma^{\mathbf{n}}$ coincides with 
$z\left(\Sigma^{\mathbf{n}}\right)$. It follows that, given an injective morphism $f:[\mathbf{m}] \rightarrow[\mathbf{n}]$, the image of $z\left(\Sigma^{\mathbf{m}}\right)$ in $\Sigma^{\mathbf{n}}$ depends only on the image of [m] in [n], i.e., only on the class of $f$ in $O(\Lambda([\mathbf{n}])$. In this way we get a system of maps $O(\Lambda[\mathbf{n}]) \rightarrow \Sigma^{\mathbf{n}}: \alpha \mapsto z(\alpha)$ possessing the property that, given an injective morphism $\gamma:[\mathbf{m}] \rightarrow[\mathbf{n}]$, one has $\Sigma(\gamma)(z(\alpha))=z(O(\gamma)(\alpha))$. For a map of partially ordered sets $\mu:[p] \rightarrow O(\Lambda[\mathbf{n}])$, where $p$ here is not $w(\mathbf{n})$, let $\theta_{\mu}$ denote the affine map $\Sigma^{p} \rightarrow \Sigma^{\mathbf{n}}$ that takes the $i$-th vertex of $\Sigma^{p}$ to $z(\mu(i))$. It follows from the construction that for an injective morphism $\gamma:[\mathbf{m}] \rightarrow[\mathbf{n}]$ and a commutative diagram of maps of partially ordered sets

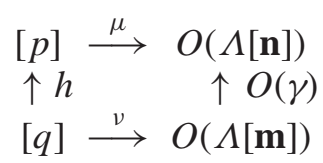

the following diagram is commutative

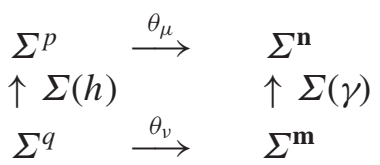

Thus, we get functorial continuous maps $|B \Lambda[\mathbf{n}]| \rightarrow \Sigma^{\mathbf{n}}$. That these are homeomorphisms is an easy exercise.

Corollary 3.11. Let $C \in \Lambda^{\circ}$ Ens. Then

(i) $|C|$ is a Hausdorff locally contractible space;

(ii) if $C$ is finite (resp. locally finite), $|C|$ is compact (resp. locally compact);

(iii) if $C$ is locally finite, any subset of $|C|$ which is a union of cells is locally contractible.

Proof. Everything follows from the corresponding properties of simplicial sets and the fact that any cell of $|C|$ is a finite union of cells of $|B C|$.

We remark that for each polysimplicial set $C$ there is a canonical surjective morphism of simplicial sets $B C \rightarrow N(O(C))$. The latter, in general, is not an isomorphism.

Lemma 3.12. If $C$ is a free polysimplicial set, then $B C \stackrel{\sim}{\rightarrow} N(O(C))$.

Proof. Since $C$ is nondegenerate, we may consider it as an object of $\Lambda^{\circ} \mathcal{E} n s$. The simplicial set $B C$ is the inductive $\operatorname{limit} \lim N(O(\Lambda[\mathbf{n}]))$ taken over all $(\Lambda[\mathbf{n}] \rightarrow C) \in \mathrm{Ob}(\Lambda / C)$. Let $\alpha: \Lambda[\mathbf{n}] \rightarrow C$ and $\alpha^{\prime}: \Lambda\left[\mathbf{n}^{\prime}\right] \rightarrow C$ be elements of $\mathrm{Ob}(\Lambda / C)$, and assume that two maps of partially ordered sets $f:[p] \rightarrow O(\Lambda[\mathbf{n}])$ and $f^{\prime}:[p] \rightarrow O\left(\Lambda\left[\mathbf{n}^{\prime}\right]\right)$ induce the same map $[p] \rightarrow O(C)$. We have two check that $f$ and $f^{\prime}$ represent the same element in the inductive limit $\lim N_{p}(O(\Lambda[\mathbf{n}]))$. First of all, if $f(p)$ is the class of a morphism $\Lambda[\mathbf{m}] \rightarrow \vec{\Lambda}[\mathbf{n}]$, then the map $f$ goes through a map of partially 
ordered sets $[p] \rightarrow O(\Lambda[\mathbf{m}])$ because the map $O(\Lambda[\mathbf{m}]) \rightarrow O(\Lambda[\mathbf{n}])$ is injective. It follows that we can replace $f$ and $f^{\prime}$ by maps that represent the same elements in the inductive limit so that $f(p)$ and $f^{\prime}(p)$ are the classes of the fundamental polysimplices $e_{\mathbf{n}}$ and $e_{\mathbf{n}^{\prime}}$. Furthermore, since the images of the latter classes in $O(C)$ coincide, there exists an isomorphism $\sigma:\left[\mathbf{n}^{\prime}\right] \stackrel{\sim}{\rightarrow}[\mathbf{n}]$ with $\alpha^{\prime}=\alpha \circ \sigma$. We may therefore replace $f^{\prime}$ and assume that $\mathbf{n}^{\prime}=\mathbf{n}$ and $\alpha^{\prime}=\alpha$. Since $C$ is free, the map of partially ordered sets $O(\Lambda[\mathbf{n}]) \rightarrow O(C)$ is injective, and the required statement follows.

We are now ready to associate with every poly-stable scheme $\mathcal{X}$ over a field $K$ a locally finite polysimplicial set $C(\mathcal{X}) \in \mathrm{Ob}\left(\Lambda^{\circ} \mathcal{E} n s\right)$ as follows. Assume first that $\mathcal{X}$ is strictly poly-stable. By Proposition 2.5, the set of strata of $\mathcal{X}$ coincides with the family of irreducible components of sets of the form $\left(\cap_{X \in A} X\right) \backslash\left(\cup_{X \notin A} X\right)$, where $A$ runs through finite subsets of $\operatorname{irr}(\mathcal{X})$, the intersection of any family of irreducible components of $\mathcal{X}$ is smooth over $K$ and, in particular, if $\mathcal{X}$ is elementary then the later intersection is always irreducible. For a point $x \in \mathcal{X}$, let $\operatorname{irr}(\mathcal{X}, x)$ denote the set of the irreducible components of $\mathcal{X}$ passing through $x$ provided with the metric with respect to which the distance between two components $X, X^{\prime} \in \operatorname{irr}(\mathcal{X}, x)$ is the codimension $\operatorname{codim}_{x}\left(X \cap X^{\prime}\right)$ of the intersection $X \cap X^{\prime}$ at the point $x$. For example, if $\mathcal{T}$ is the standard scheme $\mathcal{T}=\mathcal{T}_{0} \times \cdots \times \mathcal{T}_{p} \times \mathcal{S}$, where $\mathcal{T}_{i}=\operatorname{Spec}\left(K\left[T_{i 0}, \ldots, T_{i n_{i}}\right] /\left(T_{i 0} \cdot \ldots \cdot T_{i n_{i}}\right)\right)$ with $n_{i} \geq 1$ and $\&=\operatorname{Spec}\left(K\left[S_{1}, \ldots, S_{m}, S_{1}^{-1}, \ldots, S_{m}^{-1}\right]\right)$, then there is an isometric bijection $[\mathbf{n}] \stackrel{\sim}{\rightarrow} \operatorname{irr}(\mathcal{T}, t)$ that takes $\mathbf{j}=\left(j_{0}, \ldots, j_{p}\right) \in[\mathbf{n}]$ to the irreducible component defined by the equations $T_{0 j_{0}}=\cdots=T_{p j_{p}}=0$ and where $t$ is contained in the intersection of all irreducible components of $\mathcal{T}$. We remark that if $\varphi: \mathcal{Y} \rightarrow \mathcal{X}$ is a smooth morphism and $x \in \overline{\varphi(y)}$ for $y \in \mathcal{Y}$ then the canonical map $\operatorname{irr}(\mathcal{Y}, y) \rightarrow \operatorname{irr}(\mathcal{X}, x)$, that takes an irreducible component of $y$ to the closure of its image in $X$, is isometric and, in particular, the metric space $\operatorname{irr}(\mathcal{X}, x)$ depends only on the stratum that contains the point $x$. It follows that each étale morphism $\varphi: \mathcal{X}^{\prime} \rightarrow \mathcal{T}$ from an open elementary neighborhood $\mathcal{X}^{\prime}$ of $x$ to $\mathcal{T}$, as above, such that the image of $x$ is contained in the intersection of all irreducible components of $\mathcal{\mathcal { T }}$, gives rise to an isometric bijection $\mu_{\varphi}:[\mathbf{n}] \stackrel{\sim}{\rightarrow} \operatorname{irr}(\mathcal{X}, x)$.

Lemma 3.13. Given a pair $(x, \mu)$ consisting of a point $x \in \operatorname{str}(\mathcal{X})$ and an isometric bijection $\mu:[\mathbf{n}] \stackrel{\sim}{\rightarrow} \operatorname{irr}(\mathcal{X}, x)$, for each isometric map $f:[\mathbf{m}] \rightarrow[\mathbf{n}]$ there exists a unique pair $(y, v)$ consisting of a point $y \in \operatorname{str}(\mathcal{X})$ with $y \leq x$ and an isometric bijection $v:[\mathbf{m}] \stackrel{\sim}{\rightarrow} \operatorname{irr}(\mathcal{X}, y)$ for which the following diagram is commutative

$$
\begin{aligned}
& \text { [n] } \stackrel{\stackrel{\mu}{\rightarrow}}{\rightarrow} \operatorname{irr}(\mathcal{X}, x) \\
& \uparrow f_{v} \quad \uparrow \\
& {[\mathbf{m}] \stackrel{\sim}{\rightarrow} \operatorname{irr}(\mathcal{X}, y)}
\end{aligned}
$$


Proof. The intersection of $\cap_{X \in \operatorname{Im}(\mu \circ f)} X$ with an open elementary neighborhood of the point $x$ is irreducible, and if $y$ is the generic point of the latter, then $y \in \operatorname{str}(\mathcal{X}), y \leq x$ and $v=\mu \circ f$ induces an isometric bijection $[\mathbf{m}] \stackrel{\sim}{\rightarrow} \operatorname{irr}(\mathcal{X}, y)$. That the pair $(y, v)$ is unique is trivial.

For $[\mathbf{n}] \in \mathrm{Ob}(\Lambda)$, let $C_{\mathbf{n}}(\mathcal{X})$ denote the set of the pairs $(x, \mu)$ consisting of a point $x \in \operatorname{str}(\mathcal{X})$ and an isometric bijection $\mu:[\mathbf{n}] \stackrel{\sim}{\rightarrow} \operatorname{irr}(\mathcal{X}, x)$. By Lemma 3.13, each isometric map $f:[\mathbf{m}] \rightarrow[\mathbf{n}]$ gives rise to a map $C(f): C_{\mathbf{n}}(\mathcal{X}) \rightarrow C_{\mathbf{m}}(\mathcal{X}):(x, \mu) \mapsto(y, \nu)$. In this way we get a functor $\mathcal{X} \mapsto C(\mathcal{X})$ from the full subcategory of $\mathcal{P} s t^{\mathrm{sm}}$ consisting of strictly polystable schemes to the category $\Lambda^{\circ} \mathscr{E} n s$.

Proposition 3.14. (i) The above functor extends in a way, unique up to a unique isomorphism, to a functor $C: \mathcal{P} s t^{\mathrm{sm}} \rightarrow \Lambda^{\circ} \mathcal{E} n$ s such that for any surjective étale morphism $\mathcal{X}^{\prime} \rightarrow \mathcal{X}$ one has

$$
\operatorname{Coker}\left(C\left(\mathcal{X}^{\prime} \times_{\chi}, X^{\prime}\right) \stackrel{\rightarrow}{\rightarrow} C\left(\mathcal{X}^{\prime}\right)\right) \stackrel{\sim}{\rightarrow} C(X) ;
$$

(ii) for each poly-stable scheme $\mathcal{X}$ there are functorial isomorphisms of partially ordered sets $O(C(\mathcal{X})) \stackrel{\sim}{\rightarrow} \operatorname{str}(\mathcal{X})$ and of simplicial sets $B C(\mathcal{X}) \stackrel{\sim}{\rightarrow} N(\mathcal{X})$.

Proof. (i) To apply the construction from the proof of Proposition 2.13, it suffices to verify that $\operatorname{Coker}\left(C\left(\mathcal{X}^{\prime} \times x, \mathcal{X}^{\prime}\right) \stackrel{\rightarrow}{\rightarrow} C\left(\mathcal{X}^{\prime}\right)\right) \stackrel{\sim}{\rightarrow} C(X)$ for any surjective étale morphism of strictly poly-stable schemes $\varphi: \mathcal{X}^{\prime} \rightarrow \mathcal{X}$. First of all, that the map considered is surjective is clear. Let $\left(x_{1}, \mu_{1}\right)$ and $\left(x_{2}, \mu_{2}\right)$ be two elements of $C_{\mathbf{n}}\left(X^{\prime}\right)$ whose images in $C_{\mathbf{n}}(\mathcal{X})$ coincide. This implies that $\varphi\left(x_{1}\right)=\varphi\left(x_{2}\right)$ and, therefore, there exists a stratum of $\mathcal{X}^{\prime \prime}=\mathcal{X}^{\prime} \times X^{\prime} \mathcal{X}^{\prime}$, whose generic point $x^{\prime \prime}$ goes to $x_{i}$ under the two projections from $X^{\prime \prime}$ to $\mathcal{X}^{\prime}$. It follows that the compositions of isometric bijections $\operatorname{irr}\left(\mathcal{X}^{\prime \prime}, x^{\prime \prime}\right) \stackrel{p_{1}}{\rightarrow}$ $\operatorname{irr}\left(\mathcal{X}^{\prime}, x_{1}\right) \stackrel{\sim}{\rightarrow} \operatorname{irr}(\mathcal{X}, x)$ and $\operatorname{irr}\left(\mathcal{X}^{\prime \prime}, x^{\prime \prime}\right) \stackrel{p_{2}}{\rightarrow} \operatorname{irr}\left(\mathcal{X}^{\prime}, x_{2}\right) \stackrel{\sim}{\rightarrow} \operatorname{irr}(\mathcal{X}, x)$ coincide. This implies that the isometric bijections $p_{1}^{-1} \circ \mu_{1}$ and $p_{2}^{-1} \circ \mu_{2}$ between [n] and $\operatorname{irr}\left(\mathcal{X}^{\prime \prime}, x^{\prime \prime}\right)$ coincide and, therefore, the pair $\left(x^{\prime \prime}, p_{1}^{-1} \circ \mu_{1}\right) \in C_{\mathbf{n}}\left(\mathcal{X}^{\prime \prime}\right)$ goes to the pairs $\left(x_{1}, \mu_{1}\right)$ and $\left(x_{2}, \mu_{2}\right)$, respectively.

(ii) It suffices to construct the isomorphism for strictly poly-stable schemes. For such a scheme $\mathcal{X}$ the first isomorphism is induced by the map $(x, \mu) \mapsto x$. Furthermore, since the polysimplicial set $C(\mathcal{X})$ is free, Lemma 3.12 implies that $B C(\mathcal{X}) \stackrel{\sim}{\rightarrow} N(O(C(\mathcal{X})))$. Since $O(C(\mathcal{X})) \stackrel{\sim}{\rightarrow} \operatorname{str}(\mathcal{X})$ and $N(\mathcal{X})=N(\operatorname{str}(\mathcal{X})$, we get the required isomorphism $B C(\mathcal{X}) \stackrel{\sim}{\rightarrow} N(\mathcal{X})$.

For a poly-stable scheme $\mathcal{X}$ the nondegenerate polysimplicial set from $\Lambda^{\circ} \mathcal{E} n s$ that corresponds to $C(\mathcal{X})$ will be denoted by $\mathbf{C}(\mathcal{X})$. If $\mathcal{X}$ is strictly poly-stable, then $\mathbf{C}_{\mathbf{n}}(\mathcal{X})$ can be identified with the set of the triples $(x, I, \mu)$ consisting of a point $x \in \operatorname{str}(\mathcal{X})$, a subset $I \subset[p]$, where $p=w(\mathbf{n})$, and an isometric bijection $\mu:\left[\mathbf{n}_{I}\right] \stackrel{\sim}{\rightarrow} \operatorname{irr}(\mathcal{X}, x)$. Given a morphism $\gamma:[\mathbf{m}] \rightarrow[\mathbf{n}]$ 
associated with a triple $\left(J^{\prime}, f, \alpha\right)$, the map $\mathbf{C}(\gamma): \mathbf{C}_{\mathbf{n}}(\mathcal{X}) \rightarrow \mathbf{C}_{\mathbf{m}}(\mathcal{X})$ takes a triple $(x, I, \mu)$ to the triple $(y, J, v)$, where $J$ is the preimage of $I$ under the injective map $f: J^{\prime} \rightarrow[p]$, and the pair $(y, v)$ is defined by the pair $(x, \mu)$ and an isometric map $\left[\mathbf{m}_{J}\right] \rightarrow\left[\mathbf{n}_{I}\right]$ as in Lemma 3.13. The following lemma implies that the functor $\mathbf{C}: \mathcal{P} s t^{\mathrm{sm}} \rightarrow \Lambda^{\circ} \mathcal{E} n s$ extends naturally to the category $\mathcal{P} s t^{\mathrm{t} s}$.

Lemma 3.15. Let $\varphi: \mathcal{Y} \rightarrow \mathcal{X}$ be a trivially poly-stable morphism. Given $(y, J, v) \in \mathbf{C}_{\mathbf{n}}(\mathcal{Y})$, there exists a unique pair $(I, \mu)$ consisting of a subset $I \subset J$ and an isometric bijection $\mu:\left[\mathbf{n}_{I}\right] \stackrel{\sim}{\rightarrow} \operatorname{irr}(\mathcal{X}, x)$, where $x=\varphi(y)$, for which the following diagram is commutative

$$
\begin{gathered}
{\left[\mathbf{n}_{J}\right] \stackrel{\stackrel{\nu}{\rightarrow}}{\rightarrow} \operatorname{irr}(\mathcal{Y}, y)} \\
\downarrow \\
\quad \stackrel{\mu}{\stackrel{\mu}{\rightarrow}} \operatorname{irr}(\mathcal{X}, x)
\end{gathered}
$$

In particular, $(x, I, \mu) \in \mathbf{C}_{\mathbf{n}}(X)$.

Proof. The unicity is trivial, and the existence is an easy exercise.

The functor $\mathcal{P} s t^{\mathrm{tps}} \rightarrow \mathcal{K} e: \mathcal{X} \mapsto|\mathbf{C}(\mathcal{X})|$ will be denoted by $|\mathbf{C}|$. Notice that since the polysimplicial sets $\mathbf{C}(\mathcal{X})$ are locally finite, the topological spaces $|\mathbf{C}(\mathcal{X})|$ are locally compact. If $\mathcal{X}$ is quasi-compact, $|\mathbf{C}(\mathcal{X})|$ is compact.

Lemma 3.16. For each poly-stable scheme $X$ over $K$ and each poly-stable scheme $y$ over $K^{\prime}$ with $K^{\prime} \supset K$, there is a functorial surjective nondegenerate morphism $\mathbf{C}(\mathcal{X} \times \mathcal{Y}) \rightarrow \mathbf{C}(\mathcal{X}) \square \mathbf{C}(\mathcal{Y})$. If $\mathcal{X}$ is strictly poly-stable and all of the strata of $\mathcal{X}$ are geometrically irreducible, then the latter is an isomorphism.

Proof. To construct the morphism, we may assume that $\mathcal{X}$ and $\mathcal{y}$ are strictly poly-stable. Let $(z, I, \mu) \in \mathbf{C}_{\mathbf{n}}(\mathcal{X} \times \mathcal{Y})$, and let $\left(x, I^{\prime}, \mu^{\prime}\right)$ and $(y, J, \nu)$ be its images in $\mathbf{C}_{\mathbf{n}}(\mathcal{X})$ and $\mathbf{C}_{\mathbf{n}}(\mathcal{Y})$, respectively. It is clear that $I^{\prime} \cap J=\emptyset$ and $I^{\prime} \cup J=I$ and, therefore, the triples $\left(x, I^{\prime}, \mu^{\prime}\right)$ and $(y, J, v)$ give rise to a polysimplex in $(\mathbf{C}(\mathcal{X}) \mathbf{b}(\mathcal{C}))_{\mathbf{n}}$. This gives the required surjective nondegenerate morphism $\mathbf{C}(X \times Y) \rightarrow \mathbf{C}(X) \square \mathbf{C}(\mathcal{Y})$. If all of the strata of $\mathcal{X}$ are geometrically irreducible, then $\operatorname{str}(\mathcal{X} \times \mathcal{X}) \stackrel{\sim}{\rightarrow} \operatorname{str}(\mathcal{X}) \times \operatorname{str}(\mathcal{X})$. This implies that the above triple $(z, I, \mu)$ is uniquely defined by the triples $\left(x, I^{\prime}, \mu^{\prime}\right)$ and $(y, J, \nu)$ and, therefore, the morphism considered is injective.

Corollary 3.17. Let $X$ be a quasi-compact poly-stable scheme over $K$. Then there exists a finite separable extension $K^{\prime}$ of $K$ such that for any poly-stable scheme $\mathcal{y}$ over $K^{\prime \prime}$ with $K^{\prime \prime} \supset K^{\prime}$ one has $C(\mathcal{X} \times \mathcal{Y}) \stackrel{\sim}{\rightarrow} C(\mathcal{X} \otimes$ $\left.K^{\prime}\right) \square C(y)$. 


\section{A colored polysimplicial set associated with a poly-stable formal scheme}

In this section we introduce a category $\boldsymbol{\Lambda}_{R}$ associated with a submonoid $R \subset[0,1]$ that contains 0 and 1 . (The unit interval $[0,1]$ is considered as a monoid by multiplication; in our applications $R=|k| \cap[0,1]$.) The categories $\boldsymbol{\Lambda}_{R}$ give rise to a category of colored polysimplicial sets $\widetilde{\Lambda}^{\circ} \& n s$. We construct a commutative diagram of functors

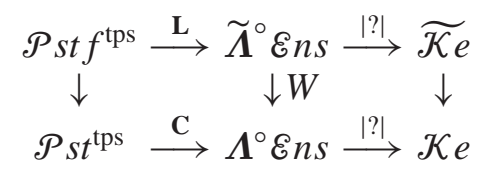

where the left vertical arrow is the functor $\mathfrak{X} \mapsto \mathfrak{X}_{s}, \widetilde{\mathcal{K} e}$ is the category of Kelley spaces endowed with a sheaf of monoids of continuous functions with values in $[0,1]$, and the right vertical arrow is the forgetful functor.

Let $R$ be a submonoid of $[0,1]$ that contains 0 and 1 , and let $\boldsymbol{\Lambda}_{R}$ be the category, whose objects are the pairs $[\mathbf{n}]_{\mathbf{r}}$ consisting of $[\mathbf{n}] \in \operatorname{Ob}(\boldsymbol{\Lambda})$ and $\mathbf{r} \in R^{[p]}$, where $p=w(\mathbf{n})$, such that $r_{0}=1$, if $[\mathbf{n}]=[0]$, and $r_{i}<1$ for all $0 \leq i \leq p$, if $[\mathbf{n}] \neq[0]$, and whose sets of morphisms $\operatorname{Hom}\left([\mathbf{m}]_{\mathbf{s}},[\mathbf{n}]_{\mathbf{r}}\right)$ consist of the morphisms $\gamma:[\mathbf{m}] \rightarrow[\mathbf{n}]$ in $\boldsymbol{\Lambda}$ which are associated with a triple $(J, f, \alpha)$ such that $s_{j}=r_{f(j)}$, for all $j \in J$. Notice that the above morphism $\gamma:[\mathbf{m}]_{\mathrm{s}} \rightarrow[\mathbf{n}]_{\mathbf{r}}$ is a composition of the projection $\pi_{J}:[\mathbf{m}]_{\mathbf{s}} \rightarrow\left[\mathbf{m}_{J}\right]_{\mathbf{s}_{J}}$ with an injective morphism $\sigma:\left[\mathbf{m}_{J}\right]_{\mathbf{s}_{J}} \rightarrow[\mathbf{n}]_{\mathbf{r}}$. (The notation $\mathbf{s}_{J}$ has the same meaning as $\mathbf{m}_{J}$.) If $\gamma$ is surjective, it has a right inverse.

The category $\boldsymbol{\Lambda}_{R}^{\circ} \mathcal{E} n s$ will be called the category of $R$-colored polysimplicial sets. If $R^{\prime}$ is a bigger submonoid of $[0,1]$ then there are fully faithful functors $\boldsymbol{\Lambda}_{R} \rightarrow \boldsymbol{\Lambda}_{R^{\prime}}$ and $\boldsymbol{\Lambda}_{R}^{\circ} \boldsymbol{E} n s \rightarrow \boldsymbol{\Lambda}_{R^{\prime}}^{\circ} \boldsymbol{E} n s$. The standard $\mathbf{r}$-colored $\mathbf{n}$-polysimplex $\boldsymbol{\Lambda}[\mathbf{n}]_{\mathbf{r}}$ is the object representable by $[\mathbf{n}]_{\mathbf{r}}$. If $L \in \mathrm{Ob}\left(\boldsymbol{\Lambda}_{R}^{\circ} \mathcal{E} n s\right)$, the image of $[\mathbf{n}]_{\mathbf{r}}$ under $L$ is denoted by $L_{\mathbf{n}}^{\mathbf{r}}$ (the set of $\mathbf{r}$-colored $\mathbf{n}$-polysimplices of $L$ ) and, for $f:[\mathbf{m}]_{\mathbf{s}} \rightarrow[\mathbf{n}]_{\mathbf{r}}$, the corresponding map $L_{\mathbf{n}}^{\mathbf{r}} \rightarrow L_{\mathbf{m}}^{\mathbf{s}}$ will be denoted by $L(f)$. One evidently has $\operatorname{Hom}\left(\boldsymbol{\Lambda}[\mathbf{n}]_{\mathbf{r}}, L\right) \stackrel{\sim}{\rightarrow} L_{\mathbf{n}}^{\mathbf{r}}$.

All of the notions and facts from $\$ 3$ that are introduced before the construction of the geometric realization functor are extended in the evident way to the category of colored polysimplicial sets. In particular, $\boldsymbol{\Lambda}_{R}^{\circ} \mathscr{E} n s$ is a symmetric strict monoidal category with respect to the multiplication $\square$ that extends the evident one on the category $\boldsymbol{\Lambda}_{R}$. Furthermore, the category of nondegenerate colored polysimplicial sets with nondegenerate morphisms between them is equivalent to $\Lambda_{R}^{\circ} \varepsilon n s$, where $\Lambda_{R}$ is the subcategory of $\Lambda_{R}$ with the same family of objects but with only injective morphisms between them. For $L \in \mathrm{Ob}\left(\Lambda_{R}^{\circ} \varepsilon n s\right)$ the corresponding nondegenerate colored polysimplicial set will be denoted by $\mathbf{L}$.

For a topological space $X$, let $\mathcal{M}_{X}$ denote the sheaf of continuous functions on $X$ with values in $[0,1]$ considered as a sheaf of monoids by multiplication. Let $\widetilde{\mathcal{K} e}$ denote the category whose objects are the pairs $\left(X, M_{X}\right)$, 
where $X$ is a Kelley space and $M_{X} \subset \mathcal{M}_{X}$ is a subsheaf of monoids, and whose morphisms $\left(Y, M_{Y}\right) \rightarrow\left(X, M_{X}\right)$ are continuous maps $\varphi: Y \rightarrow X$ such that for any function $f \in M(U)$ over an open subset $\mathcal{U} \subset X$ one has $f \circ \varphi \in M\left(\varphi^{-1}(\mathcal{U})\right)$ (i.e., the image of the sheaf $\varphi^{*} M_{X}$ in $\mathcal{M}_{Y}$ is contained in $M_{Y}$ ). Note that the category $\widetilde{\mathcal{K} e}$ admits direct and finite inverse limits and that the forgetful functor $F: \widetilde{\mathcal{K}} e \rightarrow \mathcal{K} e$ commutes with those limits. We define a functor $\Sigma_{R}: \boldsymbol{\Lambda}_{R} \rightarrow \widetilde{\mathcal{K} e}$ by $\Sigma_{R}\left([\mathbf{n}]_{\mathbf{r}}\right)=\left(\Sigma_{\mathbf{r}}^{\mathbf{n}}, M_{\mathbf{r}}^{\mathbf{n}}\right)$, where

$$
\Sigma_{\mathbf{r}}^{\mathbf{n}}=\left\{\mathbf{t}=\left(t_{i l}\right)_{0 \leq i \leq p, 0 \leq l \leq n_{i}} \in[0,1]^{[\mathbf{n}]} \mid t_{i 0} \cdot \ldots \cdot t_{i n_{i}}=r_{i}\right\}
$$

and the sheaf $M_{\mathrm{r}}^{\mathrm{n}}$ consists of all continuous functions which locally are restrictions of functions of the form $\mathbf{t} \mapsto \lambda \prod_{i=0}^{p} \prod_{l=0}^{n_{i}} t_{i l}^{a_{i l}}$ with $\lambda \in R$ and $a_{i} \in \mathbf{Z}_{+}$. (Notice that the monoid $M\left(\Sigma_{\mathbf{r}}^{\mathbf{n}}\right)$ coincides with $M\left(\stackrel{\circ}{\Sigma}_{\mathbf{r}}^{\mathbf{n}}\right)$, and each non-zero function from it has a unique representation in the above form with the property that for each $0 \leq i \leq p$ there exists $0 \leq l \leq n_{i}$ with $a_{i l}=0$.) Given a morphism $\gamma:[\mathbf{m}]_{\mathbf{s}} \rightarrow[\mathbf{n}]_{\mathbf{r}}$ associated with a triple $(J, f, \alpha)$, the map $\Sigma_{R}(\gamma)$ takes a point $\mathbf{t}=\left(t_{j k}\right)_{0 \leq j \leq q, 0 \leq k \leq m_{j}}$ to the point $\mathbf{t}^{\prime}=\left(t_{i l}^{\prime}\right)_{0 \leq i \leq p, 0 \leq l \leq n_{i}}$, where (a) if $[\mathbf{m}] \neq[0]$ and $i \notin \operatorname{Im}(f)$, or $[\mathbf{m}]=[0]$, then $t_{i l}^{\prime}=r_{i}$ for $l=\alpha_{i}(0)$ and $t_{i l}^{\prime}=1$ for $l \neq \alpha_{i}(0)$, and (b) if $[\mathbf{m}] \neq[0]$ and $i \in \operatorname{Im}(f)$, then $t_{i l}^{\prime}=t_{f^{-1}(i), \alpha_{i}^{-1}(l)}$ for $l \in \operatorname{Im}\left(\alpha_{i}\right)$ and $t_{i l}^{\prime}=1$ for $l \notin \operatorname{Im}\left(\alpha_{i}\right)$. For the map $\Sigma_{R}(\gamma)$ the image of the sheaf $\Sigma_{R}(\gamma)^{*} M_{\mathrm{r}}^{\mathbf{n}}$ in $\mathcal{M}_{\Sigma_{\mathrm{s}}^{\mathrm{m}}}$ is contained in $M_{\mathrm{s}}^{\mathrm{m}}$, i.e., $\Sigma_{R}$ is really a functor. (Notice that if $\gamma$ is injective that the set of the restrictions of functions from $\mathcal{M}\left(\Sigma_{\mathbf{r}}^{\mathbf{n}}\right)$ to the image of $\Sigma_{R}(\gamma)$ coincides with $M\left(\Sigma_{\mathrm{s}}^{\mathbf{m}}\right)$.) For brevity, we shall denote an object $\left(X, M_{X}\right)$ of the category $\widetilde{\mathcal{K} e}$ by $X$. The following fact will be used in $\S 5$.

Lemma 4.1. For $[\mathbf{m}]_{\mathrm{s}},[\mathbf{n}]_{\mathbf{r}} \in \mathrm{Ob}\left(\boldsymbol{\Lambda}_{R}\right)$, there is a canonical bijection

$$
\operatorname{Isom}\left([\mathbf{m}]_{\mathbf{s}},[\mathbf{n}]_{\mathbf{r}}\right) \stackrel{\sim}{\rightarrow} \operatorname{Isom}\left(\Sigma_{\mathbf{s}}^{\mathbf{m}}, \Sigma_{\mathbf{r}}^{\mathbf{n}}\right) \stackrel{\sim}{\rightarrow} \operatorname{Isom}\left(\stackrel{\circ}{\Sigma_{\mathbf{s}}}, \stackrel{\circ}{\Sigma_{\mathbf{r}}^{\mathbf{n}}}\right) .
$$

Proof. Both maps are evidently injective, and surjectivity of the second map easily follows from the equality $M\left(\Sigma_{\mathbf{r}}^{\mathbf{n}}\right)=M\left(\stackrel{\circ}{\Sigma}_{\mathbf{r}}^{\mathbf{n}}\right)$. It remains to check that any isomorphism $\varphi: \Sigma_{\mathbf{s}}^{\mathbf{m}} \stackrel{\sim}{\rightarrow} \Sigma_{\mathbf{r}}^{\mathbf{n}}$ is associated with an isomorphism $[\mathbf{m}]_{\mathbf{s}} \stackrel{\sim}{\rightarrow}[\mathbf{n}]_{\mathbf{r}}$. Let us call a face of $\Sigma_{\mathbf{r}}^{\mathbf{n}}$ the image of $\Sigma_{\mathbf{r}^{\prime}}^{\mathbf{n}^{\prime}}$ for some injective morphism $\left[\mathbf{n}^{\prime}\right]_{\mathbf{r}^{\prime}} \rightarrow[\mathbf{n}]_{\mathbf{r}}$. If $\left[\mathbf{n}^{\prime}\right]=[0]$ (resp. [1]) such a face is called a vertex (resp. edge). It is easy to see that a subset $A \subset \Sigma_{\mathbf{r}}^{\mathbf{n}}$ is a face if and only if there exists a function $f \in M\left(\Sigma_{\mathbf{r}}^{\mathbf{n}}\right)$ with $A=\left\{\mathbf{t} \in \Sigma_{\mathbf{r}}^{\mathbf{n}} \mid f(\mathbf{t})=1\right\}$. It follows that any isomorphism $\varphi: \Sigma_{\mathbf{s}}^{\mathbf{m}} \stackrel{\sim}{\rightarrow} \Sigma_{\mathbf{r}}^{\mathbf{n}}$ takes vertices to vertices, edges to edges and so on. We now notice that there is a canonical bijection between the set [n] and the set of vertices of $\Sigma_{\mathbf{r}}^{\mathbf{n}}$, and the distance between two elements of $[\mathbf{n}]$ is equal to the minimal number of edges which are necessary to join the corresponding vertices of $\Sigma_{\mathbf{r}}^{\mathbf{n}}$. Hence, the above isomorphism $\varphi$ induces an isometric bijection $\gamma:[\mathbf{m}] \stackrel{\sim}{\rightarrow}[\mathbf{n}]$. Replacing $\varphi$ by a composition with the evident isomorphism induced by $\gamma$, we may assume that $[\mathbf{m}]=[\mathbf{n}]$ and that the isomorphism $\gamma$ is the identity map, and we have to show that $\mathbf{s}=\mathbf{r}$ and 
$\varphi$ is the identity map. For this we notice that two functions from $M\left(\Sigma_{\mathbf{r}}^{\mathbf{n}}\right)$ that take the same values at the vertices of $\Sigma_{\mathbf{r}}^{\mathbf{n}}$ are equal. This immediately reduces the situation to the case when $[\mathbf{n}]=[1]$, i.e., $\varphi$ is an isomorphism $\Sigma_{s}^{1} \stackrel{\sim}{\rightarrow} \Sigma_{r}^{1}$ that induces the identity map on [1]. The latter easily implies that $s=r$ and $\varphi$ is the identity map.

The geometric realization functor is the functor $\mid$ ? $\mid: \Lambda_{R}^{\circ} \mathbb{E} n s \rightarrow \widetilde{\mathcal{K} e}$ which extends the functor $\Sigma_{R}$ to the one that commutes with direct limits. Lemma 4.1 implies that the automorphism group of the functor |?| is trivial. The restriction of $\Sigma_{R}$ to the category $\Lambda_{R}$ gives rise to a geometric realization functor $\mid$ ? $\mid: \Lambda_{R}^{\circ} \varepsilon n s \rightarrow \widetilde{\mathcal{K} e}$ and, for any $L \in \mathrm{Ob}\left(\Lambda_{R}^{\circ} \mathcal{E} n s\right)$, there is a canonical isomorphism $|L| \stackrel{\sim}{\rightarrow}|\mathbf{L}|$. For any pair of colored polysimplicial sets $L^{\prime}$ and $L^{\prime \prime}$ there is a canonical isomorphism $\mid L^{\prime}$ 口 $L^{\prime \prime}|\stackrel{\sim}{\rightarrow}| L^{\prime}|\times| L^{\prime \prime} \mid$. The facts stated in Lemma 3.8 and its Corollary 3.9 are straightforwardly extended to colored polysimplicial sets. In the same way one introduces the barycentric subdivision functor $B_{R}: \Lambda_{R}^{\circ} \mathcal{E} n s \rightarrow \Delta^{\circ} \mathcal{E} n s$ which extends the functor $\boldsymbol{\Lambda}_{R} \rightarrow \Delta^{\circ} \mathcal{E}_{n s}:[\mathbf{n}]_{\mathbf{r}} \mapsto N\left(O\left(\boldsymbol{\Lambda}[\mathbf{n}]_{\mathbf{r}}\right)\right)$ and commutes with direct limits. (Notice that $O\left(\boldsymbol{\Lambda}[\mathbf{n}]_{\mathbf{r}}\right) \stackrel{\sim}{\rightarrow} O(\Lambda[\mathbf{n}])$.) If in the definition of $B_{R}$ one uses the category $\Lambda_{R}$ instead of $\boldsymbol{\Lambda}_{R}$, one gets a functor $\Lambda_{R} \mathcal{E} n s \rightarrow \Delta^{\circ} \mathcal{E} n s$ compatible with $B_{R}$ with respect to the functor $L \mapsto \mathbf{L}$. As in Lemma 3.12 one shows that for a free colored polysimplicial set $L$ one has $B_{R} L \stackrel{\sim}{\rightarrow} N(O(L))$.

Lemma 4.2. For each $L \in \mathrm{Ob}\left(\Lambda_{R}^{\circ} \& n s\right)$, there is a functorial homeomorphism $\left|B_{R} L \stackrel{\sim}{\rightarrow}\right| L \mid$.

Proof. The proof is analogous to that of Lemma 3.10, we only have to indicate the points that should be changed. It suffices to construct functorial homeomorphisms $\left|B_{R} \boldsymbol{\Lambda}[\mathbf{n}]_{\mathbf{r}}\right| \stackrel{\sim}{\rightarrow} \Sigma_{\mathbf{r}}^{\mathbf{n}}$. If $\mathbf{n}=\left(n_{0}, \ldots, n_{p}\right)$ and $\mathbf{r}=\left(r_{0}, \ldots, r_{p}\right)$, let $z\left(\Sigma_{\mathbf{r}}^{\mathbf{n}}\right)$ denote the point of $\Sigma_{\mathbf{r}}^{\mathbf{n}}$ with the coordinates $t_{i l}=r_{i}^{\frac{1}{n_{i}+1}}$ for all $0 \leq i \leq p$ and $0 \leq l \leq n_{i}$. Given an injective morphism $\gamma:[\mathbf{m}]_{\mathbf{s}} \rightarrow[\mathbf{n}]_{\mathbf{r}}$, the image of $z\left(\Sigma_{\mathbf{s}}^{\mathbf{m}}\right)$ in $z\left(\Sigma_{\mathbf{r}}^{\mathbf{n}}\right)$ depends only on the image of $[\mathbf{m}]$ in $[\mathbf{n}]$, i.e., only on the class of $\gamma$ in $\left.O(\Lambda]_{\mathbf{r}}\right)$, and in this way we get a system of maps $O\left(\boldsymbol{\Lambda}[\mathbf{n}]_{\mathbf{r}}\right) \rightarrow \Sigma_{\mathbf{r}}^{\mathbf{n}}: \alpha \mapsto z(\alpha)$. We remark that for each map of partially ordered sets $\mu:[p] \rightarrow O\left(\boldsymbol{\Lambda}[\mathbf{n}]_{\mathbf{r}}\right)$ all of the points $z(\mu(i))$ are contained in an affine component of $\Sigma_{\mathbf{r}}^{\mathbf{n}}$. (Here $p$ is not $w(\mathbf{n})$.) We now define, for such a map $\mu$, a map $\theta_{\mu}: \Sigma^{p} \rightarrow \Sigma_{\mathbf{r}}^{\mathbf{n}}$ as the affine one that takes the $i$-th vertex of $\Sigma^{p}$ to $z(\mu(i))$. The remained part of the proof of Lemma 3.10 is applicable to the situation considered.

The canonical functor $\boldsymbol{\Lambda}^{\circ} \mathcal{E} n s \rightarrow \Lambda_{R}^{\circ} \mathcal{E} n s$ (induced by the functor $\boldsymbol{\Lambda}_{R} \rightarrow$ $\left.\boldsymbol{\Lambda}:[\mathbf{n}]_{\mathbf{r}} \mapsto[\mathbf{n}]\right)$ has a left adjoint functor $W: \boldsymbol{\Lambda}_{R}^{\circ} \mathcal{E} n s \rightarrow \boldsymbol{\Lambda}^{\circ} \mathcal{E} n s$. The latter extends the functor $\boldsymbol{\Lambda}_{R} \rightarrow \Lambda^{\circ} \mathcal{E} n s:[\mathbf{n}]_{\mathbf{r}} \mapsto \boldsymbol{\Lambda}$ [n] to the one that commutes with direct limits. It follows straightforwardly that the functor $W$ commutes with the multiplication $\square$ and that there are functorial isomorphisms of partially ordered sets $O(L) \stackrel{\sim}{\rightarrow} O(W L)$ and of simplicial sets $B_{R} L \stackrel{\sim}{\rightarrow} B(W L)$. 
Lemmas 3.10 and 4.2 imply that there is a functorial homeomorphism $|W L| \stackrel{\sim}{\rightarrow}|L|$. One can easily show that the canonical maps $L_{\mathbf{n}}^{\mathbf{r}} \rightarrow(W L)_{\mathbf{n}}$ give rise to a bijection $\bigsqcup_{\mathbf{r}}\left(L_{\mathbf{n}}^{\mathbf{r}}\right)^{\text {nd }} \stackrel{\sim}{\rightarrow}(W L)_{\mathbf{n}}^{\text {nd }}$. This allows one to view a colored polysimplicial set $L$ as a polysimplicial set $W L$ provided with partitions of the sets $(W L)_{\mathbf{n}}^{\text {nd }}$ (coloring of polysimplices). Notice that if $R^{\prime}$ is a bigger submonoid of $[0,1]$ then there is a commutative diagram of functors

$$
\begin{gathered}
\Lambda_{R} \& n s \quad \stackrel{L \mapsto L^{\prime}}{\longrightarrow} \boldsymbol{\Lambda}_{R^{\prime}} \& n s \\
W \searrow W^{\prime} \\
\Lambda^{\circ} \& n s
\end{gathered}
$$

In particular, the canonical morphism $|L| \rightarrow\left|L^{\prime}\right|$ is a homeomorphism. Of course, it is not an isomorphism in $\widetilde{\mathcal{K} e}$ because the sheaf of monoids on $\left|L^{\prime}\right|$ is bigger than that on $|L|$. Finally, if in the definition of $W$ one uses the category $\Lambda_{R}$ instead of $\boldsymbol{\Lambda}_{R}$, one gets a functor $\Lambda_{R}^{\circ} \mathscr{E} n s \rightarrow \Lambda^{\circ} \mathscr{E} n s$ compatible with $W$ with respect to the functor $L \mapsto \mathbf{L}$. For $L \in \mathrm{Ob}\left(\Lambda_{R}^{\circ} \varepsilon n s\right)$ one has $(W L)_{\mathbf{n}}=\coprod_{\mathbf{r}} L_{\mathbf{n}}^{\mathbf{r}}$.

We are now going to associate with a poly-stable formal scheme $\mathfrak{X}$ over $k^{\circ}$ a colored polysimplicial set $L(\mathfrak{X}) \in \mathrm{Ob}\left(\Lambda_{R}^{\circ} \mathscr{E} n s\right)$, where $R=|k| \cap[0,1]$. For this we need the following fact. Assume that $\mathfrak{X}$ is strictly poly-stable. Recall that for each point $\mathbf{x} \in \mathfrak{X}_{s}$ one can find an open neighborhood $\mathfrak{X}^{\prime}$ of $\mathbf{x}$ in $\mathfrak{X}$ and an étale morphism $\varphi: \mathfrak{X}^{\prime} \rightarrow \mathfrak{T}=\mathfrak{T}(\mathbf{n}, \mathbf{a}) \times \mathfrak{S}(m)$ such that the point $\varphi_{s}(\mathbf{x})$ is contained in the intersection of all irreducible components of $\mathfrak{T}_{s}$. The isometric bijections $\operatorname{irr}\left(\mathfrak{X}_{s}^{\prime}, \mathbf{x}\right) \stackrel{\sim}{\rightarrow} \operatorname{irr}\left(\mathfrak{X}_{s}, \mathbf{x}\right), \operatorname{irr}\left(\mathfrak{X}_{s}^{\prime}, \mathbf{x}\right) \stackrel{\sim}{\rightarrow} \operatorname{irr}\left(\mathfrak{T}_{s}, \varphi_{s}(\mathbf{x})\right)$ and $[\mathbf{n}] \stackrel{\sim}{\rightarrow} \operatorname{irr}\left(\mathfrak{T}_{s}, \varphi_{s}(\mathbf{x})\right): \mathbf{i} \mapsto \mathcal{T}_{\mathbf{i}}$ give rise to an isometric bijection $\mu_{\varphi}:[\mathbf{n}] \stackrel{\sim}{\rightarrow} \operatorname{irr}\left(\mathfrak{X}_{s}, \mathbf{x}\right): \mathbf{i} \mapsto \mathcal{X}_{\mathbf{i}}$. Notice that for any isometric bijection $\mu:[\mathbf{n}] \stackrel{\sim}{\rightarrow} \operatorname{irr}\left(\mathfrak{X}_{s}, \mathbf{x}\right)$ one can find $\varphi$ as above with $\mu_{\varphi}=\mu$.

Proposition 4.3. Given a point $\mathbf{x} \in \mathfrak{X}_{s}$ and an isometric bijection $\mu$ : $[\mathbf{n}] \stackrel{\sim}{\rightarrow} \operatorname{irr}\left(\mathfrak{X}_{s}, \mathbf{x}\right)$, there exists a tuple $\mathbf{r}=\left(r_{0}, \ldots, r_{p}\right) \in[0,1]^{[p]}$, where $p=w(\mathbf{n})$, such that for any étale morphism $\varphi: \mathfrak{X}^{\prime} \rightarrow \mathfrak{T}=\mathfrak{T}(\mathbf{n}, \mathbf{a}) \times \mathfrak{S}(m)$ as above with $\mu_{\varphi}=\mu$ one has $|\mathbf{a}|=\mathbf{r}$.

The tuple $\mathbf{r}$ from Proposition 4.3 associated with $\mu$ will be denoted by $\mathbf{r}^{\mu}$.

Proof. We first reduce the situation to the case when $p=0$ and $n_{0}=1$. Indeed, assume that in that case the required fact is true. For a fixed $0 \leq j \leq$ $p$, let $\mathbf{i}$ and $\mathbf{i}^{\prime}$ be two elements of [n] whose coordinates are distinct only at $j$-th place. Then $\operatorname{codim}\left(\mathcal{X}_{\mathbf{i}} \cap \mathcal{X}_{\mathbf{i}^{\prime}}\right)=1$, where $\mathcal{X}_{\mathbf{i}}$ is the image of $\mathbf{i}$ under $\mu$. It is easy to see that there is an open embedding of an open neighborhood of the generic point of $\mathcal{T}_{\mathbf{i}} \cap \mathcal{T}_{\mathbf{i}^{\prime}}$ to a standard formal scheme of the form $\mathfrak{T}^{\prime}=\mathfrak{T}_{j}\left(1, a_{j}\right) \times \mathfrak{S}\left(m^{\prime}\right)$. Its composition with $\varphi$ gives an étale morphism 
from an open neighborhood of the generic point of $\mathcal{X}_{\mathbf{i}} \cap \mathcal{X}_{\mathbf{i}^{\prime}}$ to $\mathfrak{T}^{\prime}$ and, therefore, $\left|a_{j}\right|$ is uniquely defined.

Thus, we may assume that $p=0$ and $n_{0}=1$, i.e., there is an étale morphism $\varphi: \mathfrak{X} \rightarrow \mathfrak{T}=\mathfrak{T}(1, a) \times \mathfrak{S}(m)$ with $\varphi(\mathbf{x}) \in \mathcal{T}_{0} \cap \mathcal{T}_{1}$. We now need the following fact which will also be used later.

Lemma 4.4. Let $\varphi: \mathfrak{X}^{\prime} \rightarrow \mathfrak{X}$ be an étale morphism of formal schemes locally finitely presented over $k^{\circ}$, and assume that $\varphi$ induces an isomorphism $\mathfrak{y}^{\prime} \stackrel{\sim}{\rightarrow} y$ between subschemes $\mathfrak{y}^{\prime} \subset \mathfrak{X}_{s}$ and $\mathcal{y} \subset \mathfrak{X}_{s}$. Then it induces an isomorphism $\pi^{-1}\left(\mathcal{y}^{\prime}\right) \stackrel{\sim}{\rightarrow} \pi^{-1}(\mathcal{y})$.

If the valuation on $k$ is discrete, the assumption implies that $\varphi$ induces an isomorphism between formal completions of $\mathfrak{X}^{\prime}$ and $\mathfrak{X}$ along $\mathfrak{y}^{\prime}$ and $\mathscr{y}$, $\mathfrak{X}_{/ y^{\prime}}^{\prime} \stackrel{\sim}{\rightarrow} \mathfrak{X} y$, and the lemma follows from the fact that $\left(\mathfrak{X}_{/ y^{\prime}}^{\prime}\right)_{\eta} \stackrel{\sim}{\rightarrow} \pi^{-1}\left(\mathcal{Y}^{\prime}\right)$ and $\left(\mathfrak{X}_{y}\right)_{\eta} \stackrel{\sim}{\rightarrow} \pi^{-1}(\mathcal{Y})$ (see [Ber6, $\left.\left.\S 1\right]\right)$. The reasoning for the previous arguments works in fact in the general case and, for completeness, we recall it.

Proof. We may assume that $\mathfrak{X}=\operatorname{Spf}(A)$ and $\mathfrak{X}^{\prime}=\operatorname{Spf}\left(A^{\prime}\right)$ are affine and that $y$ and $y^{\prime}$ are closed subschemes. Furthermore, since the canonical morphism $\mathfrak{Y}^{\prime} \rightarrow \mathfrak{X}_{s}^{\prime} \times \mathfrak{X}_{s}$ Y is an étale closed immersion, we can shrink $\mathfrak{X}^{\prime}$ and assume that $\mathcal{Y}^{\prime} \stackrel{\sim}{\rightarrow} \mathfrak{X}_{s}^{\prime} \times \mathfrak{X}_{s}$ Y. Let $f_{1}, \ldots, f_{m}$ be elements of $A$ whose reductions in $\widetilde{A}$ generate the ideal of $\mathcal{Y}$, and let $a$ be a fixed non-zero element of $k^{\circ \circ}$. (It suffices to consider the case of nontrivial valuation on $k$.) Then for any $n \geq 1$ there is an isomorphism of rings $A / J^{n} \stackrel{\sim}{\rightarrow} A^{\prime} / J^{\prime n}$, where $J=\left(a, f_{1}, \ldots, f_{m}\right)$ and $J^{\prime}=J A^{\prime}$, and therefore there is an isomorphism of completions with respect to powers of $J$ and $J^{\prime}, \widehat{A} \stackrel{\sim}{\rightarrow} \widehat{A}^{\prime}$. It remains to show that the analytic spaces $\pi^{-1}(\mathcal{y})$ and $\pi^{-1}\left(\mathcal{Y}^{\prime}\right)$ are expressible only in terms of these completions.

Recall $[\mathrm{Ber} 3, \S 1]$ that $\mathfrak{X}_{\eta}$ is the space of continuous multiplicative seminorms on $A$ that extend the valuation on $k^{\circ}$. The subspace $\pi^{-1}(y)$ is the subset of those semi-norms whose values at $f_{i}$ 's are strictly less than one. It follows that $\pi^{-1}(\mathcal{Y})$ is identified with the space of continuous multiplicative semi-norms on the completion $\widehat{A}$ that extend the valuation on $k^{\circ}$, and therefore the morphism $\pi^{-1}\left(y^{\prime}\right) \rightarrow \pi^{-1}(y)$ is a homeomorphism. Furthermore, the space $\pi^{-1}(y)$ is a union of an increasing sequence of affinoid domains $W_{1} \subset W_{2} \subset \ldots$ such that $W_{n}$ is a Weierstrass domain in $W_{n+1}$ (for example, $W_{n}=\left\{x \in \mathfrak{X}_{\eta} \| f_{i}(x) \mid \leq 1-\frac{1}{n}, 1 \leq i \leq m\right\}$ ). It follows that affinoid domains in $\pi^{-1}(\mathcal{y})$ are compact subsets $V$ for which there are a $k$-affinoid algebra $\mathcal{A}_{V}$ and a continuous homomorphism $\widehat{A} \rightarrow$ $\mathcal{A}_{V}^{\circ}$ universal with respect to the property that the image of $\mathcal{M}\left(\mathcal{A}_{V}\right)$ in $\pi^{-1}(y)$ is contained in $V$. If now $V^{\prime}$ is an affinoid domain in $\pi^{-1}\left(y^{\prime}\right)$, then the canonical continuous homomorphism $\widehat{A} \stackrel{\sim}{\rightarrow} \widehat{A}^{\prime} \rightarrow \mathcal{A}_{V^{\prime}}^{\circ}$ satisfies the universal property, and therefore $V=\varphi\left(V^{\prime}\right)$ is an affinoid domain in $\pi^{-1}(\mathcal{y})$ isomorphic to $V^{\prime}$. Hence $\pi^{-1}\left(y^{\prime}\right) \stackrel{\sim}{\rightarrow} \pi^{-1}(y)$. 
Let $\mathfrak{X}=\operatorname{Spf}(A)$ and $\mathcal{A}=A \otimes_{k^{\circ}} k$, and let $x_{i}$ be the preimage (under the reduction map) of the generic point of $\mathcal{X}_{i}, i=0,1$. It suffices to verify the following fact. For each $i=0,1$, the set $\left\{\left|f\left(x_{i}\right)\right| /|f|_{\text {sup }} \mid f \in \mathcal{A}^{*}\right\}$ coincides with the set $\left\{|a|^{j} \mid j \geq 0\right\}$, if $a \neq 0$, and with $\{1\}$, if $a=0$. Of course, we may assume that $i=1$.

Let $t_{j}$ be the preimage of the generic point of $\mathcal{T}_{j}, j=0,1$. Since $\left|\varphi^{*} T_{1}\left(x_{1}\right)\right|=\left|T_{1}\left(t_{1}\right)\right|=|a|$, it follows that the first set contains the second one. To verify the converse inclusion, assume first that $m=0$, i.e., $\mathfrak{T}=\mathfrak{T}(1, a)$, and let $\mathbf{t}=\varphi_{s}(\mathbf{x})$. One has $\widetilde{k}(\mathbf{t})=\widetilde{k}$, and $\widetilde{k}(\mathbf{x})$ is a finite separable extension of $\widetilde{k}$. Let $K$ be the finite unramified extension of $k$ with $\widetilde{K}=\widetilde{k}(\mathbf{x})$, and let $\mathfrak{T}^{\prime}=\mathfrak{T} \widehat{\otimes} K^{\circ}$ and $\mathfrak{X}^{\prime}=\mathfrak{X} \widehat{\otimes} K^{\circ}$. The preimage of $\mathbf{t}$ in $\mathfrak{T}_{s}^{\prime}$ is a point $\mathbf{t}^{\prime}$ with $\widetilde{k}\left(\mathbf{t}^{\prime}\right)=\widetilde{K}$, and the preimage of $\mathbf{x}$ in $\mathfrak{X}_{s}^{\prime \prime}$ contains a point $\mathbf{x}^{\prime}$ with $\widetilde{k}\left(\mathbf{x}^{\prime}\right)=\widetilde{K}$. By Lemma 4.4, there are isomorphisms of $k$-analytic spaces $\pi^{-1}(\mathbf{x}) \stackrel{\sim}{\rightarrow} \pi^{-1}\left(\mathbf{x}^{\prime}\right) \stackrel{\sim}{\rightarrow} \pi^{-1}\left(\mathbf{t}^{\prime}\right)$. It follows that the restriction of any element $f$ of $\mathcal{A}$ to $\pi^{-1}(\mathbf{x})$ has a unique representation in the form $\sum_{n=-\infty}^{\infty} a_{n} u_{n}$, where $u_{n}=T_{0}^{-n}$ for $n<0$ and $u_{n}=T_{1}^{n}$ for $n \geq 0, a_{n} \in K$ and, for every $r<1$, one has $\left|a_{n}\right| r^{|n|} \rightarrow 0$ as $n \rightarrow \pm \infty$. If $a=0$, then the restriction to $\pi^{-1}(\mathbf{x})$ of each $f \in \mathcal{A}^{*}$ is of the form $\lambda(1+g)$, where $\lambda \in K^{*}$ and $g$ is an analytic function on $\pi^{-1}(\mathbf{x})$ whose absolute value is strictly less than one, and therefore $\left|f\left(x_{1}\right)\right| /|f|_{\text {sup }}=\{1\}$. If $a \neq 0$, then the restriction to $\pi^{-1}(\mathbf{x})$ of each $f \in \mathcal{A}^{*}$ is of the form $\lambda u_{n}(1+g)$, where $\lambda$ and $g$ are as above and $n \in \mathbf{Z}$. One has

$$
|f|_{\text {sup }}=\sup _{x \in \pi^{-1}(\mathbf{x})}|f(x)|=|\lambda|,
$$

and, when $\mathcal{U}$ runs through open neighborhoods of the point $t_{1}$ in $\mathfrak{T}_{1}$, one has

$$
\left|f\left(x_{1}\right)\right|=\lim _{u} \sup _{x \in \pi^{-1}(\mathbf{x}) \cap \varphi_{\eta}^{-1}(u)}|f(x)|=|\lambda| \cdot|a|^{\max (-n, 0)} .
$$

It follows that $\left|f\left(x_{1}\right)\right| /|f|_{\text {sup }}=|a|^{\max (-n, 0)}$.

Assume now that $m \geq 1$. Consider the canonical morphism $\psi: \mathfrak{T} \rightarrow$ $\mathfrak{S}(m)$, and let $y$ be the maximal point of $\mathfrak{S}(m)_{\eta}$. One evidently has $\left\{t_{0}, t_{1}\right\} \subset$ $\psi^{-1}(y)$ and $\left\{x_{0}, x_{1}\right\} \subset(\varphi \psi)^{-1}(y)$, and the situation is reduced to the morphism $\mathfrak{X} \times \mathfrak{S}_{(m)} \operatorname{Spf}\left(\mathcal{H}(y)^{\circ}\right) \rightarrow \mathfrak{T} \times{ }_{\mathfrak{S}(m)} \operatorname{Spf}\left(\mathcal{H}(y)^{\circ}\right)$ which is of the type considered just now. The proposition is proven.

We are continuing to assume that $\mathfrak{X}$ is strictly poly-stable. For $[\mathbf{n}]_{\mathbf{r}} \in$ $\operatorname{Ob}\left(\Lambda_{R}\right)$, let $L_{\mathbf{n}}^{\mathbf{r}}(\mathfrak{X})$ denote the set of the polysimplices $(\mathbf{x}, \mu) \in C_{\mathbf{n}}\left(\mathfrak{X}_{s}\right)$ (i.e., $\mathbf{x} \in \operatorname{str}\left(\mathfrak{X}_{s}\right)$ and $\mu$ is an isometric bijection $\left.[\mathbf{n}] \stackrel{\sim}{\rightarrow} \operatorname{irr}\left(\mathfrak{X}_{s}, \mathbf{x}\right)\right)$ such that $\mathbf{r}^{\mu}=\mathbf{r}$. It follows from Proposition 4.3 that, given a morphism $[\mathbf{m}]_{\mathbf{s}} \rightarrow[\mathbf{n}]_{\mathbf{r}}$ in $\Lambda_{R}$, the map $C_{\mathbf{n}}\left(\mathfrak{X}_{s}\right) \rightarrow C_{\mathbf{m}}\left(\mathfrak{X}_{s}\right)$, associated to the corresponding morphism $[\mathbf{m}] \rightarrow[\mathbf{n}]$ in $\Lambda$, induces a map $L_{\mathbf{n}}^{\mathbf{r}}(\mathfrak{X}) \rightarrow L_{\mathbf{m}}^{\mathbf{s}}(\mathfrak{X})$. Thus, $L(\mathfrak{X}) \in \operatorname{Ob}\left(\Lambda_{R}^{\circ} \mathcal{E} n s\right)$, and we get a functor $\mathfrak{X} \mapsto L(\mathfrak{X})$ from the full subcategory of $k^{\circ}-\mathcal{P} s t f^{\mathrm{sm}}$ consisting of strictly poly-stable formal schemes to the category $\Lambda_{R}^{\circ} \& n s$. From Proposition 3.14 it follows that this functor 
extends in a way, unique up to a unique isomorphism, to a functor $L_{k}: k^{\circ}$ $\mathcal{P} s t f^{\mathrm{sm}} \rightarrow \Lambda_{R}^{\circ} \mathcal{E} n s$ such that for any surjective étale morphism $\mathfrak{X}^{\prime} \rightarrow \mathfrak{X}$ one has

$$
\operatorname{Coker}\left(L\left(\mathfrak{X}^{\prime} \times \mathfrak{X} \mathfrak{X}^{\prime}\right) \stackrel{\rightarrow}{\rightarrow} L\left(\mathfrak{X}^{\prime}\right)\right) \stackrel{\sim}{\rightarrow} L(\mathfrak{X}) .
$$

It follows also that there are functorial isomorphisms of polysimplicial sets $W L(\mathfrak{X}) \stackrel{\sim}{\rightarrow} C\left(\mathfrak{X}_{s}\right)$, of partially ordered sets $O\left(L(\mathfrak{X}) \stackrel{\sim}{\rightarrow} \operatorname{str}\left(\mathfrak{X}_{s}\right)\right.$ and of simplicial sets $B_{R} L(\mathfrak{X}) \stackrel{\sim}{\rightarrow} N\left(\mathfrak{X}_{s}\right)$.

Furthermore, the nondegenerate colored polysimplicial set from $\boldsymbol{\Lambda}_{R}^{\circ} \mathbb{E} n s$ that corresponds to $L(\mathfrak{X})$ will be denoted by $\mathbf{L}(\mathfrak{X})$. If $\mathfrak{X}$ is strictly poly-stable, then $\mathbf{L}(\mathfrak{X})$ can be identified with the set of the polysimplices $(\mathbf{x}, I, \mu) \in$ $\mathbf{C}\left(\mathfrak{X}_{s}\right)\left(\right.$ i.e., $\mathbf{x} \in \operatorname{str}\left(\mathfrak{X}_{s}\right), I \subset[p]$ and $\left.\mu:\left[\mathbf{n}_{I}\right] \stackrel{\sim}{\rightarrow} \operatorname{irr}\left(\mathfrak{X}_{s}, \mathbf{x}\right)\right)$ such that $\mathbf{r}^{\mu}=\mathbf{r}_{I}$. The functor $\mathbf{C}: \mathcal{P} s t^{\mathrm{tps}} \rightarrow \Lambda^{\circ} \mathcal{E} n s$ gives rise to a functor $\mathbf{L}_{k}: k^{\circ}-\mathcal{P} s t f^{\mathrm{tps}} \rightarrow$ $\Lambda_{R}^{\circ} \mathcal{E} n s$ that extends the previous one from the category $k^{\circ}-\mathcal{P} s t f^{\mathrm{sm}}$.

To consider all of the functors $\mathbf{L}_{k}$ simultaneously, it is convenient to introduce a category $\widetilde{\boldsymbol{\Lambda}}^{\circledR} \mathcal{E} n s$ whose objects are pairs $(k, L)$, where $k$ is a nonArchimedean field and $L \in \mathrm{Ob}\left(\boldsymbol{\Lambda}_{R}^{\circ} \mathcal{E} n s\right), R=|k| \cap[0,1]$, and morphisms $(K, N) \rightarrow(k, L)$ are pairs consisting of an isometric embedding $k \hookrightarrow K$ and a morphism $N \rightarrow L^{\prime}$ in $\boldsymbol{\Lambda}_{R^{\prime}}^{\circ} \& n s, R^{\prime}=|K| \cap[0,1]$. The correspondences $(k, L) \mapsto|L|$ and $(k, L) \mapsto W L$ give rise to the geometric realization functor $\mid$ ? $\mid: \widetilde{\Lambda}^{\circ} \& n s \rightarrow \widetilde{\mathcal{K} e}$ and a functor $W: \widetilde{\Lambda}^{\circ} \mathcal{E} n s \rightarrow \Delta^{\circ} \mathcal{E} n s$. For brevity, the pair $(k, L)$ will be denoted by $L$. The correspondence $(k, \mathfrak{X}) \mapsto$ $(k, \mathbf{L}(\mathfrak{X}))$ gives rise to a functor $\mathbf{L}: \mathcal{P} s t f^{\text {tps }} \rightarrow \widetilde{\Lambda}^{\circ} \mathcal{E} n s$. The functor $\mathcal{P} s t f^{\mathrm{tps}} \rightarrow \widetilde{\mathcal{K} e}: \mathfrak{X} \mapsto|\mathbf{L}(\mathfrak{X})|$ is denoted by $|\mathbf{L}|$. One evidently has a functorial isomorphism $W \mathbf{L}(\mathfrak{X}) \stackrel{\sim}{\rightarrow} \mathbf{C}\left(\mathfrak{X}_{s}\right)$ and a functorial homeomorphism $|\mathbf{L}(\mathfrak{X})| \stackrel{\sim}{\rightarrow}\left|\mathbf{C}\left(\mathfrak{X}_{s}\right)\right|$.

\section{The homotopy type of the generic fibre of a poly-stable formal scheme}

The main results of this section, Theorems 5.2-5.4, form a basis for, and are more precise than, their generalizations in $\S 8$. To formulate the first theorem, we introduce a partial ordering on the generic fibre $\mathfrak{X}_{\eta}$ of a formal scheme $\mathfrak{X}$ from $\mathcal{F}$ sch.

First of all, we introduce a partial ordering on an affinoid space $X=\mathcal{M}(\mathcal{A})$ as follows: $x \leq y$ if $|f(x)| \leq|f(y)|$ for all $f \in \mathcal{A}$.

Lemma 5.1. Let $X$ be a $k$-affinoid space and let $V$ be affinoid domain in $X$ which is a finite union of Laurent domains of the form $X\left\{r f^{-1}\right\}=\{x \in$ $X|| f(x) \mid \geq r\}$ with $r=|f|_{\text {sup. Then }}$ on $V$;

(i) the restriction of the partial ordering on $X$ to $V$ coincides with that

(ii) if $x \in V$ and $x \leq y$ for some $y \in X$, then $y \in V$. 
Proof. (ii) Assume first that $V=X\left\{r f^{-1}\right\}$. Then the inequality $x \leq y$ implies that $|f(y)| \geq|f(x)| \geq r$, and therefore $y \in X\left\{r f^{-1}\right\}$. In the general case, $V=V_{1} \cup \cdots \cup V_{n}$, where each $V_{i}$ is of the form $X\left\{r f^{-1}\right\}$. Since $x \in V_{i}$ for some $1 \leq i \leq n$, the previous case implies that $y \in V_{i} \subset V$.

(i) We remark that to verify the condition $x \leq y$ it suffices to verify that $|f(x)| \leq|f(y)|$ for all $f$ from a dense subset of $\mathcal{A}$, where $X=\mathcal{M}(\mathcal{A})$. Let $V=V_{1} \cup \cdots \cup V_{n}$, where each $V_{i}$ is of the form $X\left\{r f^{-1}\right\}$. Assume that for $x, y \in V$ one has $x \leq_{X} y$. Since $x \in V_{i}$ for some $1 \leq i \leq n$, then $y \in V_{i}$, by (ii), and therefore the situation is reduced to the case $n=1$, i.e., $V=X\left\{r f^{-1}\right\}$. By the above remark, it suffices to verify that $|h(x)| \leq|h(y)|$ for $h$ of the form $\frac{g}{f^{n}}$ with $g \in \mathcal{A}$ and $n \geq 0$. Since $|f(x)|=|f(y)|=r$ and $|g(x)| \leq|g(y)|$, it follows that $|h(x) \leq| h(y) \mid$.

We introduce a partial ordering on $\mathfrak{X}_{\eta}$ as follows: $x \leq y$ if there exists an open affine subscheme $\mathfrak{X}^{\prime} \subset \mathfrak{X}$ such that $x, y \in \mathfrak{X}_{\eta}^{\prime}$ and $x \leq y$ in $\mathfrak{X}_{\eta}^{\prime}$. From Lemma 5.1 it follows that the latter property does not depend on the choice of $\mathfrak{X}^{\prime}$, and if $x \leq y$ then $\pi(x)$ is contained in the closure of $\pi(y)$.

Theorem 5.2. One can construct for every poly-stable formal scheme $\mathfrak{X}$ a proper strong deformation retraction $\Phi: \mathfrak{X}_{\eta} \times[0,1] \rightarrow \mathfrak{X}_{\eta}:(x, t) \mapsto x_{t}$ of $\mathfrak{X}_{\eta}$ to a closed subset $S(\mathfrak{X})$, the skeleton of $\mathfrak{X}$, so that the following holds:

(i) $\left(x_{t}\right)_{t^{\prime}}=x_{\max \left(t, t^{\prime}\right)}$ for all $0 \leq t, t^{\prime} \leq 1$;

(ii) $x \leq x_{t}$ for all $0 \leq t \leq 1$; if $\mathfrak{X}$ is strictly poly-stable and $\mathfrak{X}_{\eta}$ is normal, then $x_{1}$ is a unique point from $S(\mathfrak{X})$ with $x \leq x_{1}$;

(iii) for each $x$ there exists $0 \leq t^{\prime} \leq 1$ such that $x_{t}=x$ for all $0 \leq t \leq t^{\prime}$ and the map $\left[t^{\prime}, 1\right] \rightarrow \mathfrak{X}_{\eta}: t \mapsto x_{t}$ is injective;

(iv) $\pi(x)=\pi\left(x_{t}\right)$ for all $0 \leq t<1$, and $\pi\left(x_{1}\right)$ is the generic point of the stratum of $\mathfrak{X}_{s}$ that contains $\pi(x)$;

(v) if $\mathfrak{X}_{\eta}$ is normal at $x$ then, for every open affine subscheme $\mathfrak{X}^{\prime} \subset \mathfrak{X}$ with $x \in \mathfrak{X}_{\eta}^{\prime}$ and every $0<t \leq 1$, the local ring $\mathcal{O}_{\mathfrak{X}_{\eta, x_{t}}^{\prime}}$ is a field;

(vi) if $\mathfrak{X}$ is strictly poly-stable then, for every point $x \in S(\mathfrak{X}), \widetilde{\mathscr{H}(x)}$ is a purely transcendent extension of the field $\widetilde{k}(\pi(x))$;

(vii) given a morphism $\varphi: \mathfrak{Y} \rightarrow \mathfrak{X}$ in $\mathcal{P}$ st $t^{\mathrm{tps}}$, one has $\varphi_{\eta}\left(y_{t}\right)=\varphi_{\eta}(y)_{t}$ for all $y \in \mathfrak{Y}_{\eta}$ and $0 \leq t \leq 1$; if $\varphi$ is étale then, given $x \in \mathfrak{X}_{\eta}$ and $0 \leq t<1$, each point from $\varphi_{\eta}^{-1}\left(x_{t}\right)$ is of the form $y_{t}$ for some $y \in \varphi_{\eta}^{-1}(x)$.

Let $\tau$ denote the retraction map $\mathfrak{X}_{\eta} \rightarrow S(\mathfrak{X}): x \mapsto x_{1}=\Phi(x, 1)$.

Theorem 5.3. One can construct in a unique way for every poly-stable formal scheme $\mathfrak{X}$ a monoid $P(\mathfrak{X})$ of continuous functions on $\mathfrak{X}_{\eta}$ with values in $[0,1]$ so that the following holds:

(i) if $\mathfrak{X}$ is the standard formal scheme $\mathfrak{T}(\mathbf{n}, \mathbf{a}) \times \mathfrak{S}(m)$ with $\mathbf{n}=$ $\left(n_{0}, \ldots, n_{p}\right)$, then $P(\mathfrak{X})$ consists of functions of the form $x \mapsto$ $\lambda \prod_{i=0}^{p} \prod_{j=0}^{n_{i}}\left|T_{i j}(x)\right|^{l_{i j}}$, where $\lambda \in|k| \cap[0,1]$ and $l_{i j} \in \mathbf{Z}_{+}$;

(ii) $P(\mathfrak{X})$ contains all functions of the form $x \mapsto|f(x)|$ with $f \in \mathcal{O}(\mathfrak{X}) \cap$ $\mathcal{O}\left(\mathfrak{X}_{\eta}\right)^{*}$; 
(iii) if $\tau(x)=\tau(y)$, then $f(x)=f(y)$ for all $f \in P(\mathfrak{X})$;

(iv) the correspondence $\mathfrak{X} \mapsto P(\mathfrak{X})$ is a contravariant functor on $\mathcal{P}$ st $f^{\text {tps }}$ with the property that, given a surjective étale morphism $\mathfrak{Y} \rightarrow \mathfrak{X}$, one has $P(\mathfrak{X}) \stackrel{\sim}{\rightarrow} \operatorname{Ker}(P(\mathfrak{Y}) \stackrel{\rightarrow}{\rightarrow} P(\mathfrak{Y} \times \mathfrak{X} \mathfrak{Y}))$.

We now provide as follows the skeleton $S(\mathfrak{X})$ of a poly-stable formal scheme $\mathfrak{X}$ with a subsheaf of monoids $M_{\mathfrak{X}} \subset \mathcal{M}_{S(\mathfrak{X})}$. (The skeleton $S(\mathfrak{X})$ considered as an object of the category $\widetilde{\mathcal{K}} e$ will be denoted by $\mathbf{S}(\mathfrak{X})$.) For an open subset $U \subset S(\mathfrak{X})$, a continuous function $f: U \rightarrow[0,1]$ is in $M(U)$ if for every point $x \in U$ there exist étale morphisms $\varphi_{i}: \mathfrak{X}_{i} \rightarrow \mathfrak{X}$ with affine $\mathfrak{X}_{i}$ and functions $f_{i} \in P\left(\mathfrak{X}_{i}\right), 1 \leq i \leq n$, such that the union $\cup_{i=1}^{n} \varphi_{i, \eta}\left(\mathfrak{X}_{i, \eta}\right)$ contains an open neighborhood $U^{\prime}$ of $x$ in $U$ and, for each $i$ and $y \in \varphi_{i, \eta}^{-1}\left(U^{\prime}\right)$, one has $f_{i}(y)=f\left(\varphi_{i, \eta}(y)\right)$. In this way we get a functor $\mathbf{S}: \mathcal{P} s t f^{\mathrm{tps}} \rightarrow \widetilde{\mathcal{K} e}$ that possesses the property that, given a surjective étale $\operatorname{morphism} \varphi: \mathfrak{Y} \rightarrow \mathfrak{X}$, one has $\operatorname{Coker}(\mathbf{S}(\mathfrak{Y} \times \mathfrak{X} \mathfrak{Y}) \stackrel{\vec{\rightarrow}}{\rightarrow} \mathbf{S}(\mathfrak{Y})) \stackrel{\sim}{\rightarrow} \mathbf{S}(\mathfrak{Y})$.

Theorem 5.4. There is a unique isomorphism of functors $|\mathbf{L}| \stackrel{\sim}{\rightarrow} \mathbf{S}$. Moreover, given a poly-stable formal scheme $\mathfrak{X}$, the isomorphism $|\mathbf{L}(\mathfrak{X})| \stackrel{\sim}{\rightarrow} \mathbf{S}(\mathfrak{X})$ induces a homeomorphism $|C(\mathcal{X})| \stackrel{\sim}{\rightarrow} S(\mathfrak{X}) \cap \pi^{-1}(\mathcal{X})$ for each strata subset $\mathcal{X}$ of $\mathfrak{X}_{s}$.

Here (and later) $|C(\mathcal{X})|$ denotes the union of the cells of $\left|C\left(\mathfrak{X}_{s}\right)\right|$ that correspond to the strata of $\mathfrak{X}_{s}$ contained in $\mathcal{X}$.

Recall that a formal group over $k^{\circ}$ is a formal scheme isomorphic to $k^{\circ}\left[\left[T_{1}, \ldots, T_{n}\right]\right]$ which is a group object in the category of formal schemes over $k^{\circ}$. Such a formal group $\mathfrak{G}$ gives rise to a $k$-analytic group structure on the generic fibre $\mathfrak{G}_{\eta}$ of $\mathfrak{G}$ which is isomorphic to the open unit polydisc in $\mathbf{A}^{n}$ with center at zero.

Lemma 5.5. Let $\varphi: \mathfrak{X}^{\prime} \rightarrow \mathfrak{X}$ be an étale morphism of formal schemes locally finitely presented over $k^{\circ}$. Then any action of a formal group on $\mathfrak{X}$ extends in a unique way to an action on $\mathfrak{X}^{\prime}$.

The statement and its proof hold also for special formal schemes (defined in [Ber6] only for discretely valued $k$ ).

Proof. Let $\mathfrak{G}$ be the formal scheme $\operatorname{Spf}\left(k^{\circ}\left[\left[T_{1}, \ldots, T_{n}\right]\right]\right)$ and let $i$ (resp. $\left.i^{\prime}\right)$ denote the canonical section $\mathfrak{X} \rightarrow \mathfrak{G} \times \mathfrak{X}$ (resp. $\left.\mathfrak{X}^{\prime} \rightarrow \mathfrak{G} \times \mathfrak{X}^{\prime}\right)$ of the projection $\mathfrak{G} \times \mathfrak{X} \rightarrow \mathfrak{X}$ (resp. $\left.\mathfrak{G} \times \mathfrak{X}^{\prime} \rightarrow \mathfrak{X}^{\prime}\right)$. (Direct products are taken in the category of formal schemes over $k^{\circ}$.) To prove the lemma, it suffices to show that for any morphism $m: \mathfrak{G} \times \mathfrak{X} \rightarrow \mathfrak{X}$ with $m \circ i=1 \mathfrak{X}$ there exists a unique morphism $m^{\prime}: \mathfrak{G} \times \mathfrak{X}^{\prime} \rightarrow \mathfrak{X}^{\prime}$ with $m^{\prime} \circ i^{\prime}=1_{\mathfrak{X}^{\prime}}$ that makes the following diagram commutative

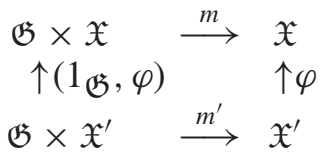


The statement is evidently local with respect to $\mathfrak{X}$ and $\mathfrak{X}^{\prime}$, and therefore we may assume that $\mathfrak{X}=\operatorname{Spf}(A)$ and $\mathfrak{X}^{\prime}=\operatorname{Spf}\left(A^{\prime}\right)$, where $A^{\prime}=B_{\{b\}}$, $B=A[S] /(P), P$ is a monic polynomial in $A[S]$ and $b$ is an element of $B$ such that the image of the derivative $P^{\prime}$ in $A^{\prime}$ is invertible. The morphism $m$ is determined by a continuous homomorphism $m^{*}: A \rightarrow$ $A\left[\left[T_{1}, \ldots, T_{n}\right]\right]$ with $m^{*}(f) \equiv f \bmod \left(T_{1}, \ldots, T_{n}\right)$ for all $f \in A$. Let $a^{\prime}$ be the image of $S$ in $A^{\prime}$. Then $P\left(a^{\prime}\right)=0$. Since the ring $A^{\prime}\left[\left[T_{1}, \ldots, T_{n}\right]\right]$ is complete in the topology defined by powers of the ideal $\left(T_{1}, \ldots, T_{n}\right)$, the pair $\left(A^{\prime}\left[\left[T_{1}, \ldots, T_{n}\right]\right],\left(T_{1}, \ldots, T_{n}\right)\right)$ is Henselian (see [Ra, Ch. XI]), and therefore there exists a unique element $\alpha^{\prime} \in A^{\prime}\left[\left[T_{1}, \ldots, T_{n}\right]\right]$ with $\alpha^{\prime} \equiv a^{\prime} \bmod \left(T_{1}, \ldots, T_{n}\right)$ and $\left(\varphi^{*} P\right)\left(\alpha^{\prime}\right)=0$. Setting $m^{\prime *}\left(a^{\prime}\right)=\alpha^{\prime}$, we get a continuous homomorphism $A^{\prime} \rightarrow A^{\prime}\left[\left[T_{1}, \ldots, T_{n}\right]\right]$ that defines the required morphism $m^{\prime}: \mathfrak{G} \times \mathfrak{X}^{\prime} \rightarrow \mathfrak{X}^{\prime}$. The uniqueness of $m^{\prime}$ follows from the uniqueness of $\alpha^{\prime}$.

Proof of Theorems 5.2-5.4. Step 1. The constructions of the first two steps are given in a more general setting than it is needed here because they will also be used in $\S 7$. Let $\mathcal{A}$ be a $k$-affinoid algebra with a fixed Banach norm. (Recall that if $\mathcal{A}$ is reduced then the spectral norm on $\mathcal{A}$ is a Banach norm.) For $\mathbf{n}=\left(n_{0}, \ldots, n_{p}\right)$ with $n_{i} \geq 1$, the algebra $\mathcal{C}=\mathcal{A}\left\{T_{00}, \ldots, T_{p n_{p}}\right\}$ is a Banach $\mathcal{A}$-module with respect to the norm $\left\|\sum_{\mu} a_{\mu} T^{\mu}\right\|=\max _{\mu}\left\|a_{\mu}\right\|$. The set $D$, consisting of the elements $\sum_{\mu} a_{\mu} T^{\mu}$ such that $a_{\mu}=0$ for all $\mu=\left(\mu_{i j}\right)_{0 \leq i \leq p, 0 \leq j \leq n_{i}}$ with $\min _{0 \leq j \leq n_{i}}\left\{\mu_{i j}\right\} \geq 1$ for some $0 \leq i \leq p$, is a Banach $\mathcal{A}$-submodule of $\mathcal{C}$. Furthermore, given elements $a_{i} \in \mathcal{A}$ with $\left\|a_{i}\right\| \leq 1$, $0 \leq i \leq p$, let $\mathbf{b}$ be the ideal of $\mathcal{C}$ generated by the elements $T_{i 0} \cdot \ldots \cdot T_{i n_{i}}-a_{i}$, $0 \leq i \leq p$. We endow the algebra $\mathscr{B}=\mathcal{C} / \mathbf{b}$ with the quotient norm, and we set $X=\mathcal{M}(\mathcal{A}), Y=\mathcal{M}(\mathscr{B})$ and

$$
S=\left\{(x, \mathbf{r}) \in X \times[0,1]^{[\mathbf{n}]}\left|r_{i 0} \cdot \ldots \cdot r_{i n_{i}}=\right| a_{i}(x) \mid, 0 \leq i \leq p\right\} .
$$

Lemma 5.6. (i) The canonical surjection $\mathcal{C} \rightarrow \mathscr{B}$ induces an isometric isomorphism $D \stackrel{\sim}{\rightarrow} B$;

(ii) given a point $s=(x, \mathbf{r}) \in S$, the function $D \rightarrow \mathbf{R}_{+}: f=$ $\sum_{\mu} a_{\mu} T^{\mu} \mapsto \max _{\mu}\left\{\left|a_{\mu}(x)\right| \mathbf{r}^{\mu}\right\}$ gives rise to a bounded multiplicative seminorm on $B$ and, therefore, it defines a point $\theta(s) \in Y$;

(iii) the mapping $S \rightarrow Y: s \mapsto \theta(s)$ is continuous and right inverse to the mapping $\phi: Y \rightarrow S$ that takes $y \in Y$ to the point $\left(x ;\left|T_{00}(y)\right|, \ldots,\left|T_{p n_{p}}(y)\right|\right)$, where $x$ is the image of $y$ in $X$;

(iv) if $\tau$ denotes the retraction map $\theta \circ \phi: Y \rightarrow \theta(S)$, then $y \leq \tau(y)$ for every point $y \in Y$;

(v) given a point $s=(x, \mathbf{r}) \in S$, let $Z$ be the Zariski closed subset of $Y$ defined by the equations $T_{i j}=0$ over all pairs $(i, j)$ with $r_{i j}=0$; then the preimage $\phi^{-1}(s)$ is an affinoid domain in $Z$ isomorphic to a direct product of $X$ and the closed annuli $A\left(r_{i j}\right)=\left\{z \in \mathbf{A}^{1}|| T(z) \mid=r_{i j}\right\}$ taken over all pairs $(i, j)$ with $r_{i j} \neq 0$. 
Proof. The statements (iii), (iv) and (v) trivially follow from (i) and (ii). By induction on $p$, to prove (i) and (ii) it suffices to consider the case $p=0$, i.e., we may assume that $C=\mathcal{A}\left\{T_{0}, \ldots, T_{n}\right\}$ and $\mathcal{B}=\mathcal{C} /\left(T_{0} \cdot \ldots \cdot T_{n}-a\right)$. That the map $D \rightarrow \mathscr{B}$ is surjective is trivial, and so to verify (i) it suffices to check that $\|f\| \leq\left\|f+\left(T_{0} \ldots \cdot T_{n}-a\right) g\right\|$ for all $f=\sum_{\mu} a_{\mu} T^{\mu} \in D$ and $g=\sum_{\mu} b_{\mu} T^{\mu} \in \mathcal{C}$. If the latter is not true, then for each $\mu$ with $\|f\|=\left\|a_{\mu}\right\|$ one has $\left\|a_{\mu}\right\|=\left\|a b_{\mu}\right\| \leq\left\|b_{\mu}\right\|$. Since the monomial $b_{\mu} T^{\mu}\left(T_{0} \ldots \cdot T_{n}\right)$ is not cancelled with other monomials in $f+\left(T_{0} \cdot \ldots \cdot T_{n}-\right.$ a) $g$, the norm of the latter is at least $\left\|b_{\mu}\right\| \geq\|f\|$.

To verify (ii), we may assume that $X=\mathcal{M}(k)$, and we have to check that, given $r_{0}, \ldots, r_{n} \in[0,1]$ with $r_{0} \cdot \ldots \cdot r_{n}=|a|$, the function $D \rightarrow$ $\mathbf{R}_{+}: f \mapsto|f|=\max _{\mu}\left\{\left|a_{\mu}\right| r^{\mu}\right\}$ gives rise to a multiplicative semi-norm on $\mathscr{B}$. If $f=\sum_{\mu} a_{\mu} T^{\mu}$ and $g=\sum_{\mu} b_{\mu} T^{\mu}$, then the image of $f \cdot g$ in $\mathscr{B}$ is represented by the element $\sum_{\delta} c_{\delta} T^{\delta} \in D$ with $c_{\delta}=\sum_{l=0}^{\infty}\left(\sum a_{\mu} b_{v}\right) a^{l}$, where the inner sum is taken over all pairs $\mu, v$ with $\min _{0 \leq i \leq n}\left\{\mu_{i}+v_{i}\right\}=l$ and $\mu+v-(l, \ldots, l)=\delta$. The sets $A=\left\{\mu|| f|=| a_{\mu} \mid r^{\mu}\right\}$ and $B=\{v|| g \mid=$ $\left.\left|b_{v}\right| r^{\nu}\right\}$ are finite subsets in the union of the coordinate hyperplanes in $\mathbf{Z}_{+}^{[n]} \subset$ $\mathbf{R}^{[n]}$. To verify the equality $|f \cdot g|=|f| \cdot|g|$, it suffices to check that there exists a line $L$ in $\mathbf{R}^{[n]}$ parallel to the diagonal line $\left\{\left(x_{0}, \ldots, x_{n}\right) \mid x_{i}=x_{j}\right.$ for all $0 \leq i, j \leq n\}$ and such that the intersection $(A+B) \cap L$ consists of one point. For this we consider the mapping $\mathbf{Z}_{+}^{[n]} \rightarrow \mathbf{Z}^{n}:\left(x_{0}, \ldots, x_{n}\right) \mapsto$ $\left(x_{1}-x_{0}, \ldots, x_{n}-x_{0}\right)$. This mapping is injective on the union of the coordinate hyperplanes, and the necessary fact is equivalent to the following one. Given two finite subsets $A, B \subset \mathbf{Z}^{n}$, there exists a point $z \in A+B$ which is represented in a unique way in the form $z=x+y$ with $x \in A$ and $y \in B$. If $x$ and $y$ are the maximal points of $A$ and $B$ with respect to the lexicographic ordering on $\mathbf{Z}^{n}$, then $z=x+y$ is such a point.

Step 2. We are still in the situation of Step 1. Let $\mathrm{G}_{\mathrm{m}}$ be the onedimensional $k$-affinoid torus $\left\{x \in \mathbf{A}^{1}|| T(x) \mid=1\right\}$. If $\mathrm{G}_{\mathrm{m}}^{(n)}$ denotes the kernel of the multiplication homomorphism $\mathrm{G}_{\mathrm{m}}^{n+1} \rightarrow \mathrm{G}_{\mathrm{m}}$, then the $k$-analytic group $G=\mathrm{G}_{\mathrm{m}}^{\left(n_{0}\right)} \times \cdots \times \mathrm{G}_{\mathrm{m}}^{\left(n_{p}\right)}$ acts in the evident way on $Y$ over $X$. For $0 \leq t \leq 1$, let $G_{t}$ denote the subgroup of $G$ defined by the inequalities $\left|\left(T_{i j}-1\right)(x)\right| \leq t$. The group $G_{t}$ for $0<t<1$ (resp. $G_{1}=G$ ) is isomorphic, as a $k$-analytic space, to a closed polydisc of radius $t$ (resp. a direct product of closed annuli of radius 1) and, therefore, it has a maximal point $g_{t}$ (resp. $g_{1}$ ). The group $G_{0}$ consists of the unit 1 of $G$, and we set $g_{0}=1$. The points $g_{t}$ are peaked ones and the map $[0,1] \rightarrow G: t \mapsto g_{t}$ is continuous and, therefore, one can construct a continuous homotopy $\Phi: Y \times[0,1] \rightarrow Y:(y, t) \mapsto y_{t}:=g_{t} * y$ (see [Ber1, §5.2 and §6.1]). It is easy to give an explicit formula for the homotopy $\Phi$ (see [Ber1, 6.1.3]). Namely, for $f \in D$ one has

$$
\left|f\left(y_{t}\right)\right|=\max _{\nu}\left|\partial_{\nu} f(y)\right| t^{\nu}
$$


where $\partial_{\nu}$ is the bounded linear operator $\frac{1}{\nu !} T^{\nu} \frac{d^{v}}{d T^{v}}: \mathcal{C} \rightarrow \mathcal{C}$

$$
\partial_{\nu}\left(\sum_{\mu} a_{\mu} T^{\mu}\right)=\sum_{\mu=v}^{\infty}\left(\begin{array}{c}
\mu \\
v
\end{array}\right) a_{\mu} T^{\mu} .
$$

The above formula implies that $y_{1}=\tau(y)$ for all $y \in Y$, i.e., $\Phi$ is a strong deformation retraction of $Y$ to the subset $\theta(S)$. Furthermore, given a point $y \in Y$, let $t^{\prime}$ be the maximum value of $t$ with the property $\left|\partial_{v} f(y)\right| t^{\nu} \leq|f(y)|$ for all $v$ and $f$. Then $y_{t}=y$ for all $0 \leq t \leq t^{\prime}$ and the map $\left[t^{\prime}, 1\right] \rightarrow Y$ : $t \mapsto y_{t}$ is injective.

Step 3. We verify here some of the properties stated in the theorems for the standard formal scheme $\mathfrak{T}=\mathfrak{T}(\mathbf{n}, \mathbf{a}) \times \mathfrak{S}(m)$ over $k^{\circ}$ with $\mathbf{n}=\left(n_{0}, \ldots, n_{p}\right)$.

(1) Let $g_{\mathbf{m}}$ be the multiplicative group $\operatorname{Spf}\left(k^{\circ}\left\{T, \frac{1}{T}\right\}\right)$ considered as a group object of the category $k^{\circ}-\mathcal{F} s c h$. (The generic fibre of $g_{m}$ is the group $\mathrm{G}_{\mathrm{m}}$ from Step 2.) The group $g_{\mathrm{m}}^{m}$ acts in the evident way on $\mathfrak{S}(m)$, and the group $g_{\mathbf{m}}^{(n)}$, the kernel of the multiplication homomorphism $g_{\mathrm{m}}^{n+1} \rightarrow g_{\mathrm{m}}$, acts in the evident way on $\mathfrak{T}(n, a)$. This gives rise to an action of $g=g_{\mathbf{m}}^{\left(n_{0}\right)} \times \ldots g_{\mathbf{m}}^{\left(n_{p}\right)} \times g_{\mathbf{m}}^{m}$ on the standard formal scheme $\mathfrak{T}$ and, therefore, to an action of the $k$-analytic group $G=g_{\eta}$ on the $k$-analytic space $\mathfrak{T}_{\eta}$. The group $G$ is of the form considered in Step 2, and so we can consider its subgroups $G_{t}$ with maximal points $g_{t}$, and a continuous homotopy $\Phi: \mathfrak{T}_{\eta} \times[0,1] \rightarrow \mathfrak{T}_{\eta}:(x, t) \mapsto x_{t}=g_{t} * x$. By Step $2, \Phi$ is a strong deformation retraction of $\mathfrak{T}_{\eta}$ to the closed subset $\theta(S)$, and it possesses the property (iii) of Theorem 5.2.

(2) The subset $\theta(S)$ is naturally to be taken as the skeleton $S(\mathfrak{T})$ of $\mathfrak{T}$. If $\tau$ is the retraction map $\theta \circ \phi: \mathfrak{T}_{\eta} \rightarrow S(\mathfrak{T})$ then, for each point $x \in S(\mathfrak{T}), \tau^{-1}(x)$ is an affinoid domain in the intersection of the irreducible components of $\mathfrak{T}_{\eta}$ that contain $x$, it is isomorphic to a direct product of closed annuli, and $x$ is the maximal point in it. Notice that if $\mathfrak{T}_{\eta}$ is normal (i.e., none of $a_{i}$ is equal to zero and, therefore, $\mathfrak{T}_{\eta}$ is irreducible), then for any pair of distinct points $x, y \in S(\mathfrak{T})$ one can find two coordinate functions $f, g$ (of the form $T_{i j}$ ) with $|f(x)|<|f(y)|$ and $|g(x)|>|g(y)|$.

(3) We define $P(\mathfrak{T})$ as the monoid of all continuous functions of the form from Theorem 5.3(i). The description of $\tau^{-1}(x)$ for $x \in S(\mathfrak{T})$ from (2) easily implies that in the case when $\mathfrak{T}_{\eta}$ is normal the set of non-zero functions from $P(\mathfrak{T})$ coincides with the set of functions of the form $x \mapsto|f(x)|$ with $f \in$ $\mathcal{O}(\mathfrak{T}) \cap \mathcal{O}\left(\mathfrak{T}_{\eta}\right)^{*}$. The space $S$ is canonically identified with $\Sigma_{\mid \mathbf{a}}^{\mathbf{n}}$, and so the homeomorphism $\theta$ induces an an isomorphism $|\mathbf{L}(\mathfrak{T})|=\left(\Sigma_{|\mathbf{a}|}^{\mathbf{n}}, M\left(\Sigma_{|\mathbf{a}|}^{\mathbf{n}}\right)\right) \stackrel{\sim}{\rightarrow}$ $(S(\mathfrak{T}), P(\mathfrak{T}))$. To verify that it induces homeomorphisms $|C(\mathcal{X})| \stackrel{\sim}{\rightarrow} S(\mathfrak{T}) \cap$ $\pi^{-1}(\mathcal{X})$ from Theorem 5.4, it suffices to assume that $\mathcal{X}$ is a stratum, i.e., there are non-empty subsets $A_{i} \subset\left[n_{i}\right]$ such that $\mathcal{X}=\left\{\mathbf{x} \in \mathfrak{T}_{s} \mid\right.$ for each $0 \leq i \leq p, T_{i j}(\mathbf{x})=0$ if $j \in A_{i}$ and $T_{i j}(\mathbf{x}) \neq 0$ if $\left.j \notin A_{i}\right\}$. Then 
$|C(\mathcal{X})|=\left\{\left(t_{i j}\right)_{0 \leq i \leq p, 0 \leq j \leq n_{i}} \in \Sigma_{|\mathbf{a}|}^{\mathbf{n}} \mid\right.$ for each $0 \leq i \leq p, t_{i j}<1$ if $j \in A_{i}$ and $t_{i j}=1$ if $\left.j \notin A_{i}\right\}$, and the necessary property follows.

Step 4. Suppose we are given an étale morphism $\varphi: \mathfrak{X}=\operatorname{Spf}(A) \rightarrow \mathfrak{T}$ with $\mathfrak{T}$ from Step 3 . Let $\mathfrak{G}$ be the formal completion of the group $g$ that acts on $\mathfrak{T}$ along its unit. It is a formal group over $k^{\circ}$. By Lemma 5.5, the action of $\mathfrak{G}$ on $\mathfrak{T}$ extends in a unique way to an action on $\mathfrak{X}$ and, therefore, the action of $\mathfrak{G}_{\eta}=\cup_{t<1} G_{t}$ on $\mathfrak{T}_{\eta}$ extends to an action on $\mathfrak{X}_{\eta}$. By [Ber1, Proposition 5.2.10], for each $x \in \mathfrak{X}_{\eta}$, the point $g_{t} * x$ is the maximal one in the orbit $G_{t} x$. It follows that for $0 \leq t \leq t^{\prime}<1$ one has $g_{t} * x \leq g_{t^{\prime}} * x$ and, therefore, one can define a point $\tau(x) \in \mathfrak{X}_{\eta}$ by

$$
\mid f\left(\tau(x)\left|=\sup _{t<1}\right| f\left(g_{t} * x\right) \mid\right.
$$

for all $f \in \mathcal{A}=A \otimes_{k^{\circ}} k$. We now define a mapping $\Phi: \mathfrak{X}_{\eta} \times[0,1] \rightarrow$ $\mathfrak{X}_{\eta}:(x, t) \mapsto x_{t}$ by $x_{t}=g_{t} * x$ if $t<1$ and $x_{1}=\tau(x)$. From [Ber1, Corollary 6.1.2], it follows that the restriction of $\Phi$ to $\mathfrak{X}_{\eta} \times[0,1$ [ is continuous. It follows from the construction that $\varphi_{\eta}\left(x_{t}\right)=\varphi_{\eta}(x)_{t}$ for all $x \in \mathfrak{X}_{\eta}$ and $t \in[0,1]$ and, in particular, $\Phi$ possesses the property (iii) of Theorem 5.2. We also remark that since $g_{t} * g_{t^{\prime}}=g_{\max \left(t, t^{\prime}\right)}$, it follows that $\left(x_{t}\right)_{t^{\prime}}=x_{\max \left(t, t^{\prime}\right)}$ for all $t, t^{\prime} \in\left[0,1\left[\right.\right.$. We claim that $g_{t} * \tau(x)=\tau(x)$ for all $x \in \mathfrak{X}_{\eta}$ and $t \in\left[0,1\left[\right.\right.$ (and, in particular, $\tau(\tau(x))=\tau(x)$ and $\left(x_{t}\right)_{t^{\prime}}=x_{\max \left(t, t^{\prime}\right)}$ for all $\left.t, t^{\prime} \in[0,1]\right)$. Indeed, one has

$$
\varphi_{\eta}\left(g_{t} * \tau(x)\right)=g_{t} * \varphi_{\eta}(\tau(x))=g_{t} * \tau\left(\varphi_{\eta}(x)\right)=\tau\left(\varphi_{\eta}(x)\right)=\varphi_{\eta}(\tau(x)) .
$$

Since the mapping $\left[0,1\left[\rightarrow \mathfrak{X}_{\eta}: t \mapsto g_{t} * \tau(x)\right.\right.$ is continuous and takes values in the finite discrete set $\varphi_{\eta}^{-1}\left(\varphi_{\eta}(\tau(x))\right)$, we get $g_{t} * \tau(x)=\tau(x)$.

We define the skeleton $S(\mathfrak{X})$ of $\mathfrak{X}$ as the image of the map $\tau$. We do not yet know that the map $\tau$ is continuous and the set $S(\mathfrak{X})$ is closed in $\mathfrak{X}_{\eta}$.

Step 5. In the situation of Step 4, the mapping $\Phi$ does not depend on the choice of the étale morphism $\varphi$. Let $\varphi^{\prime}: \mathfrak{X} \rightarrow \mathfrak{T}^{\prime}=\mathfrak{T}\left(\mathbf{n}^{\prime}, \mathbf{n}^{\prime}\right) \times \mathfrak{S}\left(m^{\prime}\right)$ be another étale morphism with $\mathbf{n}^{\prime}=\left(n_{0}^{\prime}, \ldots, n_{p^{\prime}}^{\prime}\right)$ and $\mathbf{a}^{\prime}=\left(a_{0}^{\prime}, \ldots, a_{p^{\prime}}^{\prime}\right)$, $\mathfrak{G}^{\prime}$ the corresponding formal group acting on $\mathfrak{T}^{\prime}$ by Step 4 , and $g_{t}^{\prime}$ the maximal element of the corresponding subgroup $G_{t}^{\prime}$ in the generic fibre of $\mathfrak{G}^{\prime}$. We have to verify that $g_{t}^{\prime} * x=g_{t} * x$ for all $x \in \mathfrak{X}_{\eta}$ and $0<t<1$. Since, for any non-Archimedean field $K$ over $k$, the $*$-multiplication commutes with the canonical map $\mathfrak{X}_{\eta} \widehat{\otimes} K \rightarrow \mathfrak{X}_{\eta}$ [Ber1, Proposition 5.2.8], we can increase the field $k$ and assume that the point $x$ is $k$-rational and, in particular, that the point $\mathbf{x}=\pi(x)$ is $\widetilde{k}$-rational. Furthermore, since $g_{t}^{\prime} * x$ and $g_{t} * x$ are the maximal points of the orbits $G_{t}^{\prime} x$ and $G_{t} x$, it suffices to verify that $G_{t}^{\prime} x=G_{t} x$.

First of all we list several cases when the required fact is easily verified.

(1) $\mathfrak{X}=\mathfrak{T}, \varphi=1 \mathfrak{T}$, and $\varphi^{\prime}$ is induced by an isomorphism $[\mathbf{n}] \stackrel{\sim}{\rightarrow}\left[\mathbf{n}^{\prime}\right]$ in $\Lambda$, i.e., if this isomorphism is represented by a triple ( $[p], f, \alpha)$, then $\varphi^{\prime}$ is induced by the isomorphisms $\mathfrak{T}\left(n_{i}, a_{i}\right) \stackrel{\sim}{\rightarrow} \mathfrak{T}\left(n_{f(i)}, a_{f(i)}\right)$ that take the coordinate function $T_{f(i), l}$ to $T_{i, \alpha_{i}(l)}$. 
(2) $\mathfrak{X}=\mathfrak{T}, \varphi=1_{\mathfrak{T}},\left[\mathbf{n}^{\prime}\right]=[\mathbf{n}], a_{i}^{\prime}=\lambda_{i} a_{i}$ for $\left|\lambda_{i}\right|=1,0 \leq i \leq p$, and $\varphi^{\prime}$ is induced by the isomorphisms $\mathfrak{T}\left(n_{i}, a_{i}\right) \stackrel{\sim}{\rightarrow} \mathfrak{T}\left(n_{i}, \lambda_{i} a_{i}\right)$ that takes the coordinate function $T_{i l}$ to $\lambda_{i}^{-1} T_{i 0}$ for $l=0$ and to $T_{i l}$ for $1 \leq l \leq n_{i}$.

(3) $\mathfrak{X}=\mathfrak{T}^{\prime}=\mathfrak{T}, \varphi=1_{\mathfrak{T}}$, and $\varphi^{\prime}$ is induced by an automorphism of $\mathfrak{S}(m)$ which is a composition of an automorphism generated by a permutation of the coordinate functions $S_{j}$ and of an automorphism that takes $S_{j}$ to $\lambda_{j} S_{j}$ for some $\lambda_{j} \in k$ with $\left|\lambda_{j}\right|=1$.

(4) $\mathfrak{X}$ is an open subscheme of $\mathfrak{T}$ and $\varphi^{\prime}$ is an open embedding $\mathfrak{X} \rightarrow$ $\mathfrak{T}^{\prime}=\mathfrak{T}\left(\mathbf{n}^{\prime}, \mathbf{a}^{\prime}\right) \times \mathfrak{S}\left(m^{\prime}\right)$ of the following form. Assume that $T_{i l}(\mathbf{x})=0$ for $0 \leq l \leq n_{i}^{\prime}$ and $T_{i l}(\mathbf{x}) \neq 0$ for $n_{i}^{\prime}+1 \leq l \leq n_{i}$ and that $n_{i}^{\prime} \geq 1$ exactly for $0 \leq i \leq p^{\prime}$. If $\mathfrak{T}\left(n_{i}, a_{i}\right)^{\prime}$ denotes the open subscheme of $\mathfrak{T}\left(n_{i}, a_{i}\right)$ where the functions $T_{i l}$ for $0 \leq i \leq p$ and $n_{i}^{\prime}+1 \leq l \leq n_{i}$ are invertible, then $\mathfrak{X}=\mathfrak{T}\left(n_{0}, a_{0}\right)^{\prime} \times \mathfrak{T}\left(n_{p}, a_{p}\right)^{\prime} \times \mathfrak{S}(m), \mathbf{n}^{\prime}=\left(n_{0}^{\prime}, \ldots, n_{p^{\prime}}^{\prime}\right), \mathbf{a}^{\prime}=\left(a_{0}, \ldots, a_{p^{\prime}}\right)$, $m^{\prime}=m+\sum_{i=0}^{p}\left(n_{i}-n_{i}^{\prime}\right)$, and $\varphi^{\prime}$ is the open embedding of $\mathfrak{X}$ to $\mathfrak{T}^{\prime}$ which is induced by the open embeddings $\mathfrak{T}\left(n_{i}, a_{i}\right)^{\prime} \rightarrow \mathfrak{T}\left(n_{i}^{\prime}, a_{i}\right) \times \mathfrak{S}\left(n_{i}-n_{i}^{\prime}\right)$ that take $T_{i l}$ to $T_{i 0} \cdot T_{n_{i}^{\prime}+1} \ldots \cdot T_{n_{i}}$ for $l=0$ and to $T_{i l}$ for $1 \leq l \leq n_{i}^{\prime}$, and $S_{j}$ to $T_{n_{i}^{\prime}+j}$ for $1 \leq j \leq n_{i}-n_{i}^{\prime}$.

Consider now the general case. Using Proposition 4.3 and replacing $\varphi$ and $\varphi^{\prime}$ by their compositions with morphisms of the form (1)-(4), we may assume that $\varphi$ and $\varphi^{\prime}$ are two étale morphisms to the same standard formal scheme $\mathfrak{T}=\mathfrak{T}(\mathbf{n}, \mathbf{a}) \times \mathfrak{S}(m)$ and such that the both points $\varphi_{s}(\mathbf{x})$ and $\varphi_{s}^{\prime}(\mathbf{x})$ coincide with the point $\mathbf{y}$ defined by the condition that all of $T_{i l}$ 's are equal to zero and all of $S_{j}$ 's are equal to one at $y$. Since the point $\mathbf{x}$ is $\widetilde{k}$-rational, Lemma 4.4 implies that $\varphi$ and $\varphi^{\prime}$ induce two isomorphisms $\pi^{-1}(\mathbf{x}) \stackrel{\sim}{\rightarrow} \pi^{-1}(\mathbf{y})$. The analytic space $\pi^{-1}(0)$ is easily described. For example, if $[\mathbf{n}]=[0]$, i.e., $\mathfrak{T}=\mathfrak{S}(m)$, then $\pi^{-1}(0)$ is isomorphic to the $m$-dimensional open unit disc with center at zero $D^{m}$, and the equality $G_{t}^{\prime} x=G_{t} x$ follows from the fact any analytic automorphism of the open unit polydisc takes a smaller closed polydisc to a closed polydisc of the same radius (see [Ber1, Lemma 6.4.4]). The case $[\mathbf{n}] \neq[0]$ follows from the following lemma.

Lemma 5.7. Given $m \geq 0, \mathbf{n}=\left(n_{0}, \ldots, n_{p}\right)$ and $\mathbf{a}=\left(a_{0}, \ldots, a_{p}\right)$ with $p \geq 0, n_{i} \geq 1$ and $a_{i} \in k^{\circ \circ}$ for all $0 \leq i \leq p$, let $X=X(\mathbf{n}, \mathbf{a}, m)$ be the closed analytic subset of the open unit polydisc $D^{|\mathbf{n}|+p+1+m}$ defined by the equations $T_{i 0} \cdot \ldots \cdot T_{i n_{i}}=a_{i}$ for $0 \leq i \leq p$, and let $\varphi$ be an analytic automorphism of $X$. Then

(i) $\varphi$ preserves the monoid $P(X)$ of functions on $X$ of the form $x \mapsto$ $\lambda \prod_{i=0}^{p} \prod_{l=0}^{n_{i}}\left|T_{i l}(x)\right|^{b_{i l}}$ with $\lambda \in|k| \cap[0,1]$ and $b_{i l} \in \mathbf{Z}_{+}$; moreover $P(X)$ contains the monoid $P^{*}(X)$ of functions of the form $x \mapsto|f(x)|$ with $f \in \mathcal{O}(X)^{*}$ and $|f(x)| \leq 1$ for all $x \in X$;

(ii) $\varphi\left(E_{t}(\alpha)\right)=E_{t}(\varphi(\alpha))$ for any k-rational point $\alpha=\left(\alpha_{i l}, \alpha_{j}\right) \in X$ and any $0<t<1$, where $E_{t}(\alpha)=\left\{x \in X|| T_{i l}(x)-\alpha_{i l}|\leq t| \alpha_{i l} \mid\right.$ and $\left|S_{j}(x)-\alpha_{j}\right| \leq t$ for all $\left.i, l, j\right\}$.

Note that the automorphism $\varphi$ is not required to be a $k$-analytic one. 
Proof. (i) Let $I=\left\{i \in[p] \mid a_{i}=0\right\}$. First, we claim that if $I=\emptyset$ then $P(X)=P^{*}(X) \cup\{0\}$. Indeed, the fibres of the map $\left.X \rightarrow \stackrel{\circ}{\Sigma} \mathbf{n}\right|^{\mathbf{n}}: x \mapsto$ $\left(\left|T_{i l}(x)\right|\right)_{0 \leq i \leq p, 0 \leq l \leq n_{i}}$ are analytic domains of $X$ isomorphic to a direct product of annuli of the form $\left\{x \in \mathbf{A}^{1}|| T(x) \mid=r\right\}$ with $D^{m}$. All invertible functions on these analytic domains are easily described, and the claim follows. Thus, assume that $I \neq \emptyset$. Notice that if $I=[p]$ then any function from $P(X)$ which does not vanish anywhere is a constant. Using the canonical projection $X \rightarrow Y=X\left(\mathbf{n}_{J}, \mathbf{a}_{J}, m\right)$, where $J=[p] \backslash I$, one deduces that $P^{*}(X)=P^{*}(Y)$ and, therefore, $P^{*}(X)$ coincides with the family of functions of the form $x \mapsto \lambda \prod_{i \notin I} \prod_{l=0}^{n_{i}}\left|T_{i l}(x)\right|^{b_{i l}}$ with $\lambda \in\left|k^{*}\right| \cap[0,1]$ and $b_{i l} \in \mathbf{Z}_{+}$. It follows that the latter family is preserved by $\varphi$. Furthermore, the correspondence $\mathbf{j} \mapsto X_{\mathbf{j}}=\left\{x \in X \mid T_{i j_{i}}=0\right.$ for all $\left.i \in I\right\}$ is a bijection between $\left[\mathbf{n}_{I}\right]$ and the set of irreducible components of $X$. The group $\operatorname{Aut}\left(\left[\mathbf{n}_{I}\right]\right)$ acts transitively on the latter set, and so we may assume that $\varphi$ preserves all of the irreducible components of $X$. We claim that in this case for each $i \in I$ and $0 \leq l \leq n_{i}$ one has $\varphi^{*} t_{i l}=t_{i l} f_{i l}$, where $t_{i l}$ is the function $x \mapsto\left|T_{i l}(x)\right|$ and $f_{i l} \in P^{*}(X)$. Indeed, each irreducible component $Z$ of $X$ is isomorphic to $Y \times D^{n}$ with $Y$ as above and $n=\left|\mathbf{n}_{I}\right|$, and the automorphism $\varphi$ preserves the closed analytic subsets $Z_{i}=\left\{z \in Z \mid T_{i}(z)=0\right\}, 1 \leq i \leq n$. We have to check that $\varphi^{*} t_{i}=t_{i} f_{i}$ for all $1 \leq i \leq n$, where $t_{i}$ is the function $z \mapsto\left|T_{i}(z)\right|$ and $f_{i} \in P^{*}(Y)$. Let $\varphi^{*} T_{i} \equiv f_{0}+\sum_{j=1}^{n} f_{j} T_{j}(\bmod \operatorname{deg} 2)$ with $f_{i} \in \mathcal{O}(Y)$. Since $\varphi$ preserves the sets $Z_{1} \cap \cdots \cap Z_{n}$ and $Z_{1} \cap \cdots \cap Z_{j-1} \cap Z_{j+1} \cap \cdots \cap Z_{n}$, it follows that $f_{j}=0$ for all $j \neq i$, i.e., $\varphi^{*} T_{i} \equiv f_{i} T_{i}(\bmod \operatorname{deg} 2)$. Notice that $\left|f_{i}(y)\right| \leq 1$ for all $y \in Y$. Similarly, one has $\left(\varphi^{-1}\right)^{*} T_{i} \equiv g_{i} T_{i}(\bmod \operatorname{deg} 2)$ with $g_{i} \in \mathcal{O}(Y)$. If $\varphi^{*} g_{i} \equiv h_{i}(\bmod \operatorname{deg} 1)$, we get $T_{i}=\varphi^{*}\left(\varphi^{-1}\right)^{*} T_{i} \equiv h_{i} f_{i} T_{i}(\bmod \operatorname{deg} 2)$ and, therefore, $f_{i} \in \mathcal{O}(Y)^{*}$, i.e., the claim is true.

(ii) Consider the surjective continuous mapping $\tau: X \rightarrow \stackrel{\circ}{\Sigma}_{|\mathbf{a}|}^{\mathbf{n}}: x \mapsto$ $\left(\left|T_{i l}(x)\right|\right)_{0 \leq i \leq p, 0 \leq l \leq n_{i}}$. The family $P(X)$ coincides with that of the preimages of functions from $M\left(\stackrel{\circ}{\Sigma}_{|\mathbf{a}|}^{\mathbf{n}}\right)$. Moreover, the fibres of $\tau$ are precisely the equivalence classes of the following equivalence relation on $X: x \sim x^{\prime}$ if $f(x)=f(y)$ for all $x \in P(X)$. It follows from (i) that $\varphi$ induces an automorphism of the pair $\left(\stackrel{\circ}{\Sigma}_{|\mathbf{a}|}^{\mathbf{n}}, M\left(\stackrel{\circ}{\Sigma}_{|\mathbf{a}|}^{\mathbf{n}}\right)\right)$. By Lemma 4.1 , we can replace $\varphi$ by its composition with the automorphism of $X$ associated with an automorphism of $[\mathbf{n}]_{|\mathbf{a}|}$ so that $\varphi$ induces the trivial automorphism of $\stackrel{\circ}{\Sigma}_{|\mathbf{a}|}^{\mathbf{n}}$. In this case $\varphi$ takes the set $\tau^{-1}(\tau(\alpha))$ to itself. Replacing again $\varphi$ by its composition with an automorphism of the form $\left(z_{i l}, z_{j}\right) \mapsto\left(\lambda_{i l} z_{i l}, z_{j}\right)$ for some $\lambda_{i l} \in k^{\circ}$ with $\lambda_{i 0} \cdot \ldots \cdot \lambda_{i n_{i}}=1,0 \leq i \leq p$, we may assume that $\varphi$ induces an analytic automorphism of the set $\left\{x \in \tau^{-1}(\tau(\alpha))|| T_{i l}(x)-\alpha_{i l}|<| \alpha_{i l} \mid\right.$ for all $0 \leq i \leq p$ and $\left.0 \leq l \leq n_{i}\right\}$. This set is isomorphic to the open unit polydisc $D^{n}$ for some $n$. We may assume that $\alpha=0$. Let $\varphi^{*} T_{i} \equiv \sum_{j=1}^{n} a_{i j} T_{j}(\bmod \operatorname{deg} 2)$. Using the last reasoning from the proof of (i), one shows that the matrix $\left(a_{i j}\right)$ lies in $\mathrm{GL}_{n}\left(k^{\circ}\right)$. Since the radii of open polydiscs in $D^{n}$ are invariant under linear transformations from $\mathrm{GL}_{n}\left(k^{\circ}\right)$, 
we may assume $\varphi^{*} T_{i} \equiv T_{i}(\bmod \operatorname{deg} 2)$. In this case the required fact is evident.

Step 6. In the situation of Step 4, suppose that $\mathfrak{X}_{s}$ is elementary and of the same type as $\mathfrak{T}_{s}$, i.e., $\varphi$ induces a bijection $\operatorname{irr}\left(\mathfrak{X}_{s}\right) \stackrel{\sim}{\rightarrow} \operatorname{irr}\left(\mathfrak{T}_{s}\right)$. Then $\varphi$ induces an isomorphism of colored polysimplicial sets $L(\mathfrak{X}) \stackrel{\sim}{\rightarrow} L(\mathfrak{T})=\Lambda[\mathbf{n}]_{|\mathbf{a}|}$. We claim that

(a) $S(\mathfrak{X})=\varphi_{\eta}^{-1}(S(\mathfrak{T}))$;

(b) $\varphi$ induces a homeomorphism $S(\mathfrak{X}) \stackrel{\sim}{\rightarrow} S(\mathfrak{T})$;

(c) for every point $x \in S(\mathfrak{X}), \widetilde{\mathscr{H}(x)}$ is a purely transcendent extension of the field $\widetilde{k}(\pi(x))$.

(1) One has $S(\mathfrak{X}) \subset \varphi_{\eta}^{-1}(S(\mathfrak{T}))$. Indeed, if $x \in S(\mathfrak{X})$, then $x=\tau(x)$ and, therefore, $\varphi_{\eta}(x)=\varphi_{\eta}(\tau(x))=\tau\left(\varphi_{\eta}(x)\right)$, i.e., $\varphi_{\eta}(x) \in S(\mathfrak{T})$.

(2) The statements $(a)-(c)$ are true if $m=0$, i.e., $\mathfrak{T}=\mathfrak{T}(\mathbf{n}, \mathbf{a})$. We prove by induction on $|\mathbf{n}|=n_{0}+\cdots+n_{p}$. Assume that the statement is true for smaller values of $|\mathbf{n}|$. The intersection of all of the irreducible components of $\mathfrak{X}_{s}\left(\operatorname{resp} . \mathfrak{T}_{s}\right)$ is a closed point $\mathbf{x}\left(\right.$ resp. t) and $\varphi_{s}^{-1}(\mathbf{t})=\{\mathbf{x}\}$. One has $\widetilde{k}(\mathbf{t})=\widetilde{k}$, and $\widetilde{k}(\mathbf{x})$ is a finite separable extension of $\widetilde{k}$. Let $K$ be the finite unramified extension of $k$ with $\widetilde{K}=\widetilde{k}(\mathbf{x})$, and let $\mathfrak{T}^{\prime}=\mathfrak{T}_{\mathrm{\otimes}^{\circ}} K^{\circ}$ and $\mathfrak{X}^{\prime}=\mathfrak{X} \widehat{\otimes}_{k^{\circ}} K^{\circ}$. The preimage of $\mathbf{t}$ in $\mathfrak{T}_{s}^{\prime}$ is a point $\mathbf{t}^{\prime}$ with $\widetilde{k}\left(\mathbf{t}^{\prime}\right)=\widetilde{K}$, and the preimage of $\mathbf{x}$ in $\mathfrak{X}_{s}^{\prime}$ contains a point $\mathbf{x}^{\prime}$ with $\widetilde{k}\left(\mathbf{x}^{\prime}\right)=\widetilde{K}$. By Lemma 4.4, there are isomorphisms of $k$-analytic spaces $\pi^{-1}(\mathbf{x}) \stackrel{\sim}{\rightarrow} \pi^{-1}\left(\mathbf{x}^{\prime}\right) \stackrel{\sim}{\rightarrow} \pi^{-1}\left(\mathbf{t}^{\prime}\right)$. Since $S\left(\mathfrak{T}^{\prime}\right) \cap \pi^{-1}\left(\mathbf{t}^{\prime}\right)$ is the preimage of $S(\mathfrak{T}) \cap \pi^{-1}(\mathbf{t})$ and homeomorphic to it (it is the interior of the polysimplex $\left.S(\mathfrak{T})=\Sigma_{|\mathbf{a}|}^{\mathbf{n}}\right)$, it follows that $S(\mathfrak{X}) \cap \pi^{-1}(\mathbf{x})$ is also the preimage of $S(\mathfrak{T}) \cap \pi^{-1}(\mathbf{t})$ and homeomorphic to it. It follows also that to verify (c) for points from $S(\mathfrak{X}) \cap \pi^{-1}(\mathbf{x})$ it suffices to check that, for every point $t \in S(\mathfrak{T}) \cap \pi^{-1}(\mathbf{t}), \widetilde{\mathscr{H}(x)}$ is a purely transcendent extension of $\widetilde{k}$. The latter is a consequence of the following lemma.

Lemma 5.8. Let $x$ be the maximal point of the closed polydisc of radius $\left(r_{1}, \ldots, r_{n}\right)$ with center at zero in $\mathbf{A}^{n}$. Then the field $\widetilde{\mathcal{H}(x)}$ is a purely transcendent extension of $\widetilde{k}$.

Proof. Using the projection $\mathbf{A}^{n} \rightarrow \mathbf{A}^{n-1}$ to the first $n-1$ coordinates, the statement is reduced by induction to the case $n=1$. Of course, we may assume that the radius $r$ of the disc is positive. The field $\widetilde{\mathcal{H}(x)}$ is generated over $\widetilde{k}$ by the residues of $f(x)$ for polynomials $f=\sum_{i=0}^{\infty} a_{i} T^{i}$ with $|f(x)|=\max \left|a_{i}\right| r^{i} \leq 1$.

Case $r \in \sqrt{|k|}$. Let $n$ be the minimal positive integer with $r^{n} \in|k|$, and let $r^{n}=|\alpha|$ for some $\alpha \in k^{*}$. If $i$ is not divisible by $n$, then the inequality $\left|a_{i}\right| r^{i} \leq 1$ implies the more strong inequality $\left|a_{i}\right| r^{i}<1$ and, therefore, we may assume that $f=\sum_{i=0}^{\infty} a_{n i} T^{n i}=\sum_{i=0}^{\infty} a_{n i} \alpha^{j}\left(\frac{T^{n}}{\alpha}\right)^{i}$. It 
follows that $\widetilde{\mathscr{H}(x)}$ is generated over $\widetilde{k}$ by the residue of $\frac{T^{n}}{\alpha}$. The latter is evidently transcendent over $\widetilde{k}$.

Case $r \notin \sqrt{|k|}$. In this case the inequality $\left|a_{i}\right| r^{i} \leq 1$ implies $\left|a_{i}\right| r^{i}<1$ for all non-zero $i$ 's and, therefore, $\widetilde{\mathcal{H}(x)}=\widetilde{k}$.

To verify the statements (a)-(c) over the complement of $S(\mathfrak{X}) \cap \pi^{-1}(\mathbf{x})$ in $S(\mathfrak{X})$, we need the following lemma.

Lemma 5.9. Let $\varphi: X \rightarrow T$ be a morphism of $k$-affinoid spaces and $t \in \Gamma(T)$. Then

(i) the restriction of the partial ordering on $X$ to $X_{t}$ coincides with that on $X_{t}$;

(ii) if $x \in X_{t}$ and $x \leq y$ for some $y \in X$, then $y \in X_{t}$.

Recall that $X_{t}$ denotes the fibre of $\varphi$ at the point $t$. This is an $\mathscr{H}(t)$-analytic space whose underlying topological space is $\varphi^{-1}(t)$.

Proof. (i) Let $T=\mathcal{M}(\mathcal{A})$ and $X=\mathcal{M}(\mathscr{B})$. From [Ber1, Corollary 2.4.5], it follows that the point $t$ coincides with the intersection of all of the Laurent domains of the form $T\left\{r f^{-1}\right\}$, where $f \in \mathcal{A}$ and $r=|f|_{\text {sup. }}$. It follows that the set of functions of the form $\frac{g}{f}$, where $g \in \mathcal{B}$ and $f \in \mathcal{A}$ is such that $|f(t)|=|f|_{\text {sup }}$, is dense in $\mathscr{B} \widehat{\otimes}_{\mathscr{A}} \mathscr{H}(t)$. (The spectrum of the latter algebra is $X_{t}$.) Let $x, y \in X_{t}$, and assume that $x \leq_{X} y$. It suffices to verify that $|h(x)| \leq|h(y)|$ for $h$ of the above form $\frac{g}{f}$ but this is evident.

(ii) For every $f \in \mathcal{A}$ with $|f(t)|=|f|_{\text {sup }}$, one has $|f(y)| \leq|f|_{\text {sup }}=$ $|f(x)| \leq|f(y)|$. It follows that $|f(y)|=|f|_{\text {sup }}$, i.e., $y \in X_{t}$.

Let $t$ be a point from $S(\mathfrak{T})$ whose reduction is not contained, say, in the irreducible component of $\mathfrak{T}_{s}$ defined by the equations $T_{i n_{i}}=0,0 \leq$ $i \leq p$. Then $\left|T_{i n_{i}}(t)\right|=1$ for some $0 \leq i \leq p$. Consider the canonical morphism $\psi: \mathfrak{T} \rightarrow \mathfrak{E}=\operatorname{Spf}\left(k^{\circ}\left\{T_{i n_{i}}\right\}\right)$. Then the image of $t$ in $\mathfrak{E}_{\eta}$ is the maximal point $e$ of $\mathfrak{E}_{\eta}$. By Lemma 5.9, one has $S(\mathfrak{T}) \cap \psi_{\eta}^{-1}(e)=$ $S\left(\mathfrak{T}^{\prime}\right)$ and $S(\mathfrak{X}) \cap\left(\psi_{\eta} \varphi_{\eta}\right)^{-1}(s)=S\left(\mathfrak{X}^{\prime}\right)$, where $\mathfrak{T}^{\prime}=\mathfrak{T} \times \mathfrak{E} \operatorname{Spf}\left(\mathscr{H}(e)^{\circ}\right)$ and $\mathfrak{X}^{\prime}=\mathfrak{X} \times \mathfrak{E} \operatorname{Spf}\left(\mathscr{H}(e)^{\circ}\right)$. One has $\mathfrak{T}^{\prime}=\mathfrak{T}\left(\mathbf{n}^{\prime}, \mathbf{a}^{\prime}\right)$ (over $\left.\mathscr{H}(e)^{\circ}\right)$, where $\mathbf{n}^{\prime}=\left(n_{0}, \ldots, n_{i-1}, n_{i}-1, n_{i+1}, \ldots, n_{p}\right)$ and $\mathbf{a}^{\prime}=\left(a_{0}, \ldots, a_{i-1}, \frac{a_{i}}{T_{i n_{i}}(e)}\right.$, $\left.a_{i+1}, \ldots, a_{p}\right)$, if $n_{i} \geq 2$, and $\mathbf{n}^{\prime}=\left(n_{0}, \ldots, n_{i-1}, n_{i+1}, \ldots, n_{p}\right)$ and $\mathbf{a}^{\prime}=$ $\left(a_{0}, \ldots, a_{i-1}, a_{i+1}, \ldots, a_{p}\right)$, if $n_{i}=1$. We can therefore apply the induction hypothesis to the morphism $\mathfrak{X}^{\prime} \rightarrow \mathfrak{T}^{\prime}$. It follows that the preimage of the point $t$ in $\mathfrak{X}_{\eta}$ is one point $x$. If $x \leq y$ for some point $y \in \mathfrak{X}_{\eta}$ then $t=\varphi_{\eta}(x) \leq \varphi_{\eta}(y)$, and since $t \in S(\mathfrak{T})$ then $\varphi_{\eta}(y)=t$, and therefore $y=x$, i.e., $x \in S(\mathfrak{X})$. Hence $S(\mathfrak{X})=\varphi_{\eta}^{-1}(S(\mathfrak{T}))$ and, in particular, $S(\mathfrak{X})$ is closed in $\mathfrak{X}_{\eta}$. Since $S(\mathfrak{X}) \rightarrow S(\mathfrak{T})$ is a bijective continuous map between compact spaces, it is a homeomorphism. Since $\widetilde{\mathscr{H}(e)}=\widetilde{k}(\pi(e))$ it follows that $\widetilde{\mathscr{H}(x)}$ is purely transcendent over $\widetilde{k}(\pi(x))$.

(3) The statement is true for $m \geq 1$. Consider the canonical projection $\psi: \mathfrak{T} \rightarrow \mathfrak{S}=\mathfrak{S}(m)$. Let $s$ be the maximal point of $\mathfrak{S}_{\eta}$, and set $\mathfrak{T}^{\prime}=\mathfrak{T} \times \mathfrak{S}$ 
$\operatorname{Spf}\left(\mathscr{H}(s)^{\circ}\right)$ and $\mathfrak{X}^{\prime}=\mathfrak{X} \times_{\mathfrak{S}} \operatorname{Spf}\left(\mathscr{H}(s)^{\circ}\right)$. Since $S(\mathfrak{T}) \subset \psi_{\eta}^{-1}(s)$, it follows from (1) that $S(\mathfrak{X}) \subset\left(\psi_{\eta} \varphi_{\eta}\right)^{-1}(s)$. By Lemma 5.9, we get $S(\mathfrak{T})=S\left(\mathfrak{T}^{\prime}\right)$ and $S(\mathfrak{X})=S\left(\mathfrak{X}^{\prime}\right)$. Since $\widetilde{\mathscr{H}(s)}=\widetilde{k}(\pi(s))$, the statement is reduced to the case $m=0$.

Thus, we get a homeomorphism $|L(\mathfrak{X})| \stackrel{\sim}{\rightarrow}|L(\mathfrak{T})| \stackrel{\sim}{\rightarrow} S(\mathfrak{T}) \stackrel{\sim}{\rightarrow} S(\mathfrak{X})$. It possesses the property of Theorem 5.4 because the homeomorphism $|L(\mathfrak{T})| \stackrel{\sim}{\rightarrow} S(\mathfrak{T})$ possesses that property and $\pi_{\mathfrak{T}}\left(\varphi_{\eta}(x)\right)=\varphi_{s}\left(\pi_{\mathfrak{X}}(x)\right)$ for all $x \in \mathfrak{X}_{\eta}$.

Step 7. In the situation of Step 4, the mapping $\Phi: \mathfrak{X}_{\eta} \times[0,1] \rightarrow \mathfrak{X}_{\eta}$ is continuous (and, in particular, $\Phi$ is a strong deformation retraction of $\mathfrak{X}_{\eta}$ to $S(\mathfrak{X})$ ). Indeed, the statement is local with respect to the Zariski topology of $\mathfrak{X}$ and, therefore, we may assume that we are in the situation of Step 6. In this case the claim follows from the following simple fact.

Lemma 5.10. Let $\varphi: Y \rightarrow X$ be a proper map of topological spaces. Assume we are given a continuous map $\Phi: X \times[0,1] \rightarrow X:(x, t) \mapsto x_{t}$ and a map $\Psi: Y \times[0,1] \rightarrow Y:(y, t) \mapsto y_{t}$ such that the restriction of $\Psi$ to $Y \times\left[0,1\left[\right.\right.$ is continuous, $\varphi\left(y_{t}\right)=\varphi(y)_{t}$ and $\varphi^{-1}\left(\varphi\left(y_{1}\right)\right)=\left\{y_{1}\right\}$ for all $y \in Y$ and $t \in[0,1]$. Then the map $\Psi$ is continuous.

Proof. It suffices to verify that $\Psi$ is continuous at each point $(y, 1) \in$ $Y \times[0,1]$. Let $\mathcal{V}$ be an open neighborhood of the point $y_{1}$. Since $\varphi$ is proper and $\varphi^{-1}\left(\varphi\left(y_{1}\right)\right)=\left\{y_{1}\right\}$, there exists an open neighborhood of the point $\varphi\left(y_{1}\right)$ with $\varphi^{-1}(\mathcal{U}) \subset \mathcal{V}$. By continuity of $\Phi$, we can find an open neighborhood $\mathcal{W}$ of $\varphi\left(y_{1}\right)$ and a number $\varepsilon>0$ with $\Phi(\mathcal{W} \times[1-\varepsilon, 1]) \subset \mathcal{U}$. It follows that $\Psi\left(\varphi^{-1}(\mathcal{W}) \times[1-\varepsilon, 1]\right) \subset \mathcal{V}$, i.e., $\Psi$ is continuous.

Step 8. Recall (see [En, §2.4]) that a surjective continuous map of topological spaces $f: Y \rightarrow X$ is said to be a factor map if the canonical map $Y / E(f) \rightarrow X$ is a homeomorphism. (Here $Y / E(f)$ is the quotient space of $Y$ with respect to the equivalence relation $E(f)$ defined by the partition $\left\{f^{-1}(x)\right\}_{x \in X}$.) In other words, $f: Y \rightarrow X$ is a factor map if it induces a homeomorphism $\operatorname{Coker}\left(Y \times_{X} Y \stackrel{\rightarrow}{\rightarrow} Y\right) \stackrel{\sim}{\rightarrow} X$. We claim that, given a surjective étale morphism $\varphi: \mathfrak{Y} \rightarrow \mathfrak{X}$ between formal schemes locally finitely presented over $k^{\circ}, \varphi_{\eta}: \mathfrak{Y}_{\eta} \rightarrow \mathfrak{X}_{\eta}$ is a factor map. Indeed, by [Ber3, $\S \S 2-3]$, the latter is a quasi-étale covering of $\mathfrak{X}_{\eta}$, and the claim follows from the following lemma.

Lemma 5.11. Any quasi-étale covering of analytic spaces $\varphi: Y \rightarrow X$ is a factor map.

Proof. We may assume that $X$ is compact (for example, affinoid). By the definition of a quasi-étale covering, each point of $X$ has a neighborhood of the form $\varphi\left(V_{1}\right) \cup \cdots \cup \varphi\left(V_{n}\right)$ for some affinoid domains $V_{1}, \ldots, V_{n} \subset Y$. It follows that there exists a surjective morphism $\varphi^{\prime}: Y^{\prime} \rightarrow X$ with compact $Y^{\prime}$ which factors through the morphism $\varphi$. Since $\varphi^{\prime}$ is evidently a factor map, then so is $\varphi$ (see [En, 2.4.5]). 
Step 9. Let $\mathfrak{X}$ be a poly-stable formal scheme over $k^{\circ}$. Take a surjective étale morphism $\psi: \mathfrak{X}^{\prime} \rightarrow \mathfrak{X}$, where $\mathfrak{X}^{\prime}$ is a disjoint union of strictly poly-stable affine formal schemes each of whom admits an étale morphism to a standard formal scheme. By Step 7, there is a continuous mapping $\Phi: \mathfrak{X}_{\eta}^{\prime} \times[0,1] \rightarrow \mathfrak{X}_{\eta}^{\prime}:\left(x^{\prime}, t\right) \mapsto x_{t}^{\prime}$. We claim that the mapping $\Phi:$ $\mathfrak{X}_{\eta} \times[0,1] \rightarrow \mathfrak{X}_{\eta}:(x, t) \mapsto x_{t}:=\psi_{\eta}\left(x_{\eta}^{\prime}\right)$, where $x^{\prime} \in \psi^{-1}(x)$, is well defined, continuous, proper and does not depend on the choice of $\mathfrak{X}^{\prime}$ and $\psi$. Indeed, take a surjective étale morphism $\mathfrak{X}^{\prime \prime} \rightarrow \mathfrak{X}^{\prime} \times \mathfrak{X} \mathfrak{X}^{\prime}$ with $\mathfrak{X}^{\prime \prime}$ satisfying the same properties as $\mathfrak{X}^{\prime}$. If $\widetilde{x}^{\prime}$ is another point from $\psi^{-1}(x)$, then there exists a point $x^{\prime \prime} \in \mathfrak{X}_{\eta}^{\prime \prime}$ with $p_{1}\left(x^{\prime \prime}\right)=x^{\prime}$ and $p_{2}\left(x^{\prime \prime}\right)=\tilde{x}^{\prime}$, where $p_{1}$ and $p_{2}$ are the two projections from $\mathfrak{X}_{\eta}^{\prime \prime}$ to $\mathfrak{X}_{\eta}^{\prime}$. By Step $5, p_{1}\left(x_{t}^{\prime \prime}\right)=x_{t}^{\prime}$ and $p_{2}\left(x_{t}^{\prime \prime}\right)=\widetilde{x}_{t}^{\prime}$ and, therefore, the images of $x_{t}^{\prime}$ and $\widetilde{x}_{t}^{\prime}$ in $\mathfrak{X}_{\eta}$ coincide, i.e., the mapping $\Phi$ is well defined. That it is continuous easily follows from Lemma 5.11 and the fact that it is continuous for $\mathfrak{X}^{\prime}$. Step 5 implies that $\Phi$ does not depend on the choice of $\mathfrak{X}^{\prime}$ and $\psi$. To see the properness of $\Phi$, it suffices to take $\mathfrak{X}^{\prime}$ such that the images of the connected components of $\mathfrak{X}^{\prime}$ in $\mathfrak{X}$ form a locally finite covering. Since $\Phi$ is proper for such $\mathfrak{X}^{\prime}$, it is also proper for $\mathfrak{X}$.

Thus, we can define a continuous map $\tau: \mathfrak{X}_{\eta} \rightarrow \mathfrak{X}_{\eta}$ by $\tau(x)=\Phi(x, 1)$ and the skeleton of $\mathfrak{X}$ by $S(\mathfrak{X})=\tau\left(\mathfrak{X}_{\eta}\right)$. The previous steps imply that the properties (i), (ii), (iii) and (vi) of Theorem 5.2 are true. The first part of (vii) for morphisms from $\mathcal{P} s t f^{\text {ét }}$ is also true.

Step 10. The statement (iv) of Theorem 5.2 is true. The first part of the statement follows from the fact that the action of a formal group on a formal scheme is trivial on the closed fibre. The second part is local with respect to the étale topology of $\mathfrak{X}$ and, therefore, it suffices to consider the case of the standard formal scheme $\mathfrak{T}$ from Step 3 . We have to verify that for a stratum $\mathcal{T}$ of $\mathfrak{T}_{s}$ with generic point $\mathbf{t}$ one has $S(\mathfrak{T}) \cap \pi^{-1}(\mathcal{T}) \subset \pi^{-1}(\mathbf{t})$. As in Step 3(3), there are non-empty subsets $A_{i} \subset\left[n_{i}\right]$ such that $\mathcal{T}=\left\{\mathbf{t}^{\prime} \in \mathfrak{T}_{s} \mid\right.$ for each $0 \leq i \leq p, T_{i j}\left(\mathbf{t}^{\prime}\right)=0$ if $j \in A_{i}$ and $T_{i j}\left(\mathbf{t}^{\prime}\right) \neq 0$ if $\left.j \notin A_{i}\right\}$. Consider the canonical morphism $\psi: \mathfrak{T} \rightarrow \mathfrak{E}=\operatorname{Spf}\left(k^{\circ}\left\{T_{i j}\right\}_{0 \leq i \leq p, j \notin A_{i}}\right)$. The image of $\mathbf{t}$ is the generic point $\mathbf{e}$ of the closed fibre of $\mathfrak{E}$, and $\psi_{s}^{-1}(\mathbf{e})=\{\mathbf{t}\}$. One also has $\pi^{-1}(\mathbf{e})=\{e\}$, where $e$ is the maximal point of $\mathfrak{E}_{\eta}$, and $\psi_{\eta}\left(S(\mathfrak{T}) \cap \pi^{-1}(\mathcal{T})\right)=\{e\}$. From Lemma 5.9 it follows that $S(\mathfrak{T}) \cap \pi^{-1}(\mathcal{T}) \subset$ $S\left(\mathfrak{T}^{\prime}\right)$, where $\mathfrak{T}^{\prime}=\mathfrak{T} \times \mathfrak{E} \operatorname{Spf}\left(\mathcal{H}(e)^{\circ}\right)$. The latter is a standard formal scheme over $\mathscr{H}(e)^{\circ}$ such that $\mathbf{t}$ is the generic point of its maximal stratum. The inclusion $S(\mathfrak{T}) \cap \pi^{-1}(\mathcal{T}) \subset \pi^{-1}(\mathbf{t})$ follows.

Step 11. The statement ( $v$ ) of Theorem 5.2 is true. First of all, we remark that, given a $k$-affinoid space $X$, a non-Archimedean field $k^{\prime}$ over $k$ and an affinoid domain $X^{\prime}$ in $X \widehat{\otimes} k^{\prime}$, for any point $x^{\prime} \in X^{\prime}$ the local ring $\mathcal{O}_{X^{\prime}, x^{\prime}}$ is a faithfully flat over $\mathcal{O}_{X, x}$, where $x$ is the image of $x^{\prime}$ in $X$ (see [Ber1, 2.2.4(ii)] and [Ber2, 2.1.3]). It follows that we can shrink $\mathfrak{X}$ and increase the ground field, so that the situation is as in Step 6 and the point $x$ is $k$-rational. Consider first the case $t<1$. Then $\mathbf{x}:=\pi(x)=\pi\left(x_{t}\right), \pi^{-1}(\mathbf{x})$ is 
open in $\mathfrak{X}_{\eta}$ and $\pi^{-1}(\mathbf{x}) \stackrel{\sim}{\rightarrow} \pi^{-1}\left(\varphi_{s}(\mathbf{x})\right)$. It follows that the normality locus of $\pi^{-1}(\mathbf{x})$ is isomorphic to an open subset of $\mathbf{A}^{|\mathbf{n}|+m}$ and $G_{t} x$ is isomorphic to a closed polydisc of radius $t$ there. The maximal point of the latter polydisc evidently possesses the required property. Consider now the case $t=1$, and set $y=x_{1}=\tau(x)$. By Step 6, $\varphi_{\eta}^{-1}\left(\varphi_{\eta}(y)\right)=\{y\}$. It follows that $X:=\tau^{-1}(y)$ is a regular strictly $k$-affinoid domain in $\mathfrak{X}_{\eta}$ with the unique maximal point $y$. Assume that there is an element $f \in \mathcal{O}_{X, y}$ with $f(y)=0$. The function $f$ is analytic in a small affinoid neighborhood of $y$ in $X$ of the form $Y=X\left(r g^{-1}\right)$, where $g \in \mathcal{O}(X)$ and $r<r^{\prime}:=|g|_{\text {sup. }}$. But the point $y$ is also the unique maximal point of the affinoid domain $Y^{\prime}=X\left(r^{\prime} g^{-1}\right)$ and, therefore, $f=0$ in $\mathcal{O}\left(Y^{\prime}\right)$. Since $\mathcal{O}_{Y^{\prime}, y}$ is faithfully flat over $\mathcal{O}_{X, y}$, it follows that $f$ is zero in $\mathcal{O}_{X, y}$.

Step 12. The statement (vii) of Theorem 5.2 is true. Indeed, since its first part is known to be true for morphisms from $\mathcal{P} s t f^{\text {ét }}$, to check it for arbitrary morphisms from $\mathcal{P} s t f^{\mathrm{tps}}$ it suffices to consider only the case of the canonical projection $\mathfrak{T}^{\prime} \times \mathfrak{T}^{\prime \prime} \rightarrow \mathfrak{T}^{\prime}$, where $\mathfrak{T}^{\prime}$ and $\mathfrak{T}^{\prime \prime}$ are standard formal schemes. But in this case, the required fact is easily seen from the construction of the maps $\tau$ and $\Phi$. As for the second part of (vii), we notice that if it is true for the morphism $\mathfrak{Y} \widehat{\otimes}_{k^{\circ}} K^{\circ} \rightarrow \mathfrak{X} \widehat{\otimes}_{k^{\circ}} K^{\circ}$, where $K$ is a bigger non-Archimedean field over $k$, then it is also true for the morphism $\varphi$. We can therefore increase the ground field $k$ so that the point $\mathbf{x}=\pi(x)$ and all of the points from $\varphi_{s}^{-1}(\mathbf{x})$ are $\widetilde{k}$-rational. In this case from Lemma 4.4 it follows that $\varphi$ induces isomorphisms $\pi^{-1}(\mathbf{y}) \stackrel{\sim}{\rightarrow} \pi^{-1}(\mathbf{x})$ for all points $\mathbf{y} \in \varphi_{s}^{-1}(\mathbf{x})$, and the statement is obviously true.

Thus, Theorem 5.2 is already proven.

Step 13. In the situation of Step 6, assume that $\mathfrak{X}_{s}$ is elementary. We claim that the family $P(\mathfrak{X})$ of the preimages of functions from $P(\mathfrak{T})$ does not depend on the choice of the étale morphism $\varphi$, and contains all functions of the form $x \mapsto|f(x)|$ with $f \in \mathcal{O}(\mathfrak{X}) \cap \mathcal{O}\left(\mathfrak{X}_{\eta}\right)^{*}$. The assumption implies that the map $\operatorname{irr}\left(\mathfrak{X}_{s}\right) \rightarrow \operatorname{irr}\left(\mathfrak{T}_{s}\right)$ is injective. Using the construction from Step 5(4) and Proposition 4.3, we may assume that we are given two étale morphisms $\varphi$ and $\varphi^{\prime}$ from $\mathfrak{X}$ to the same standard formal scheme $\mathfrak{T}=\mathfrak{T}(\mathbf{n}, \mathbf{a}) \times \mathfrak{S}(m)$ that induce bijections $\operatorname{irr}\left(\mathfrak{X}_{s}\right) \stackrel{\sim}{\rightarrow} \operatorname{irr}\left(\mathfrak{T}_{s}\right)$. We now use the reasoning from Step 6. Assume first that $m=0$, i.e., $\mathfrak{T}=\mathfrak{T}(\mathbf{n}, \mathbf{a})$, and let $\mathbf{x}$ and $\mathbf{t}$ be the intersections of all of the irreducible components of $\mathfrak{X}_{s}$ and $\mathfrak{T}_{s}$, respectively. Then $\varphi_{s}^{-1}(\mathbf{t})=\varphi_{s}^{\prime-1}(\mathbf{t})=\{\mathbf{x}\}$. If $K$ is the finite unramified extension of $k$ with $\widetilde{K}=\widetilde{k}(\mathbf{x}), \widetilde{T}^{\prime}=\mathfrak{T} \widehat{\mathbb{Q}}_{k^{\circ}} K^{\circ}$ and $\mathfrak{X}^{\prime}=\mathfrak{X} \widehat{\otimes}_{k^{\circ}} K^{\circ}$, then the preimage of $\mathbf{t}$ in $\mathfrak{T}_{s}^{\prime}$ is a point $\mathbf{t}^{\prime}$ with $\widetilde{k}\left(\mathbf{t}^{\prime}\right)=\widetilde{K}$, and the preimage of $\mathbf{x}$ in $\mathfrak{X}_{s}^{\prime}$ contains a point $\mathbf{x}^{\prime}$ with $\widetilde{k}\left(\mathbf{x}^{\prime}\right)=\widetilde{K}$. By Lemma 4.4 , the étale morphisms $\varphi$ and $\varphi^{\prime}$ give rise to a $k$-analytic automorphism of $\pi^{-1}\left(\mathbf{t}^{\prime}\right)$. Since the functions from $P(\mathfrak{X})$ are determined by their values on $S(\mathfrak{X}) \cap \pi^{-1}(\mathbf{x})$, the case $m=0$ of the claim follows from Lemma 5.7(i). The case $m \geq 1$ is obtained using the reasoning from Step 6(3). 
Thus, we define $P(\mathfrak{X})$ as the monoid of the preimages of functions from $P(\mathfrak{T})$. It follows from the construction that $P(\mathfrak{X})$ possesses the properties (ii) and (iii) of Theorem 5.3 and, moreover, if $\operatorname{irr}\left(\mathfrak{X}_{s}\right) \stackrel{\sim}{\rightarrow} \operatorname{irr}\left(\mathfrak{T}_{s}\right)$ then $P(\mathfrak{T}) \stackrel{\sim}{\rightarrow} P(\mathfrak{X})$.

Step 14. Theorem 5.3 is true. For a poly-stable formal scheme $\mathfrak{X}$ we take an étale covering $\left(\mathfrak{X}_{i} \rightarrow \mathfrak{X}\right)_{i \in I}$ with $\mathfrak{X}_{i}$ 's satisfying the assumptions of Step 13, and we define $P(\mathfrak{X})$ as the monoid of all continuous functions on $\mathfrak{X}_{\eta}$ whose preimage on each $\mathfrak{X}_{i, \eta}$ is contained in $P\left(\mathfrak{X}_{i}\right)$. From Step 13 it follows that $P(\mathfrak{X})$ does not depend on the choice of the étale covering. It follows from the construction that the properties (ii), (iii) and (iv) are true. The statement on the unicity follows from (i) and (iv).

Step 15. Theorem 5.4 is true. If $\mathfrak{X}$ is from Step 6, then Step 13 easily implies that the homeomorphism $S(\mathfrak{X}) \stackrel{\sim}{\rightarrow} S(\mathfrak{T})$ is in fact an isomorphism in $\widetilde{\mathcal{K}} e$ and, therefore, we get an isomorphism $|\mathbf{L}(\mathfrak{X})| \stackrel{\sim}{\rightarrow} \mathbf{S}(\mathfrak{X})$ that possesses the property of Theorem 5.4. Furthermore, by the constructions, in the general case the spaces $|\mathbf{L}(\mathfrak{X})|$ and $\mathbf{S}(\mathfrak{X})$ are direct limits of the spaces $|\mathbf{L}(\mathfrak{Y})|$ and $\mathbf{S}(\mathfrak{Y})$, respectively, taken over all étale morphisms $\mathfrak{Y} \rightarrow \mathfrak{X}$ with $\mathfrak{Y}$ 's satisfying the assumptions of Step 6 . The above isomorphisms $|\mathbf{L}(\mathfrak{Y})| \stackrel{\sim}{\rightarrow} \mathbf{S}(\mathfrak{Y})$ give rise to an isomorphism $|\mathbf{L}(\mathfrak{X})| \stackrel{\sim}{\rightarrow} \mathbf{S}(\mathfrak{X})$ that possesses the required property. That it is unique follows from Lemma 4.1.

Remark 5.12. (i) The system of homotopies $\Phi$ satisfying the properties of Theorem 5.2 is unique if one fixes them for the standard formal schemes as in Step 3. Indeed, it suffices to check the unicity of the maps $[0,1[\mapsto$ $\mathfrak{X}_{\eta}: x \mapsto x_{t}$ for every point $x \in \mathfrak{X}_{\eta}$. For this we take étale morphisms $\varphi: \mathfrak{X}^{\prime} \rightarrow \mathfrak{X}$ and $\psi: \mathfrak{X}^{\prime} \rightarrow \mathfrak{T}$ such that $x=\varphi_{\eta}\left(x^{\prime}\right)$ for some point $x^{\prime} \in \mathfrak{X}_{\eta}^{\prime}$ and $\mathfrak{T}$ is a standard formal scheme. Furthermore, using the property (vi) we can increase the ground field so that the point $x^{\prime}$ is $k$-rational. In this case $\varphi$ and $\psi$ induce isomorphisms $\pi^{-1}\left(\mathbf{x}^{\prime}\right) \stackrel{\sim}{\rightarrow} \pi^{-1}(\mathbf{x})$ and $\pi^{-1}\left(\mathbf{x}^{\prime}\right) \stackrel{\sim}{\rightarrow} \pi^{-1}(\mathbf{y})$, where $\mathbf{x}^{\prime}=\pi\left(x^{\prime}\right), \mathbf{x}=\pi(x), \mathbf{y}=\pi(y)$ and $y=\psi_{\eta}\left(x^{\prime}\right)$, and the required fact follows from the properties (iv) and (vi).

(ii) Recall that for a formal scheme $\mathfrak{X}$ the correspondence $\mathfrak{Y} \mapsto \mathfrak{Y}_{s}$ induces an equivalence between the category of formal schemes étale over $\mathfrak{X}$ and the category of schemes étale over $\mathfrak{X}_{s}$ (see [Ber3, §3]). Fix a functor $\mathfrak{Y}_{s} \mapsto \mathfrak{Y}$ inverse to the above one. Theorem 5.3 implies that for a polystable $\mathfrak{X}$ the correspondence $\mathfrak{Y}_{s} \mapsto P(\mathfrak{Y})$ is a sheaf of monoids $P_{\mathfrak{X}}$ on the étale site of $\mathfrak{X}_{s}$. Let $P_{\mathfrak{X}}^{*}$ denote the subsheaf of monoids such that $P^{*}(\mathfrak{Y})$ consists of the functions from $P(\mathfrak{Y})$ that do not vanish on $\mathfrak{Y}_{\eta}$. Assume now that the valuation on $k^{\circ}$ is discrete and $\mathfrak{X}$ is the formal completion $\widehat{X}$ of a scheme $\mathcal{X}$ over $k^{\circ}$ with semi-stable reduction. A canonical logarithmic structure on $\mathcal{X}$ is defined by the étale sheaf of monoids $\mathcal{P}_{X}=\mathcal{O}_{x} \cap j_{*} \mathcal{O}_{X}^{*}$, where $j$ is the open embedding $\mathcal{X}_{\eta} \hookrightarrow \mathcal{X}$ (see [Kato]). There is a canonical epimorphism of étale sheaves of monoids on $\mathcal{X}_{s}, i^{*} \mathcal{P}_{X} \rightarrow P_{\hat{x}}^{*}$, where $i$ is the closed immersion $\mathcal{X}_{s} \rightarrow \mathcal{X}$. 
(iii) Let $F$ be a local non-Archimedean field, $\Omega_{F}^{d}$ the Drinfeld upper halfplane of dimension $d-1$ over $F$, and $\widehat{\Omega}_{F}^{d}$ the formal scheme over $F^{\circ}$ with the generic fibre $\Omega_{F}^{d}$ considered in [Dr2] and [Mus]. The formal scheme $\widehat{\Omega}_{F}^{d}$ is strictly poly-stable, and the colored polysimplicial set $L\left(\widehat{\Omega}_{F}^{d}\right)$ gives rise to the Bruhat-Tits building $\mathscr{B}_{F}^{d}$ of the group $\mathrm{SL}_{d}(F)$. Some of the objects from Theorem 5.2-5.4 were constructed in [Ber5] in an elementary way, i.e., without using formal schemes.

(iv) Assume that a non-Archimedean field $k$ contains local fields $F_{1}, \ldots, F_{n}$. Then Theorems 5.2-5.4 and their consequences are applicable to the formal scheme $\left(\widehat{\Omega}_{F_{1}}^{d_{1}} \widehat{\otimes} k^{\circ}\right) \times \cdots \times\left(\widehat{\Omega}_{F_{n}}^{d_{n}} \widehat{\otimes} k^{\circ}\right)$, whose generic fibre is the $k$-analytic space $\left(\Omega_{F_{1}}^{d_{1}} \widehat{\otimes} k\right) \times \cdots \times\left(\Omega_{F_{n}}^{d_{n}} \widehat{\otimes} k\right)$. Analytic spaces of this form are considered in [Var].

\section{A polysimplicial set associated with a poly-stable fibration of schemes}

In this section we construct a commutative diagram of functors

$$
\begin{gathered}
{\mathcal{P} s t_{l}^{\mathrm{tps}}} \\
\mathbf{C}^{l} \swarrow \quad \operatorname{str}^{l} \\
\boldsymbol{\Lambda}^{\circ} \mathbb{E} n s \quad \stackrel{o}{\longrightarrow} \quad \mathcal{O} r
\end{gathered}
$$

where $\operatorname{str}^{l}$ associates with a poly-stable fibration $\underline{\mathcal{X}}$ the partially ordered set $\operatorname{str}\left(\mathcal{X}_{l}\right)$. For this we need the following fact which explains, in particular, the necessity of consideration of the category $\boldsymbol{\Lambda}$ instead of $\Lambda$. (The extension of all constructions from the category $\mathcal{P} s t f^{\mathrm{sm}}$ to $\mathcal{P} s t f^{\text {tps }}$ is only a by-product not important for our applications.)

Lemma 6.1. Let $\varphi: \mathcal{Y} \rightarrow \mathcal{X}$ be a strictly poly-stable morphism with strictly pluri-nodal $\mathcal{X}$, and let $x, x^{\prime} \in \operatorname{str}(\mathcal{X})$ be points with $x^{\prime} \leq x$. Consider the map of partially ordered sets $\operatorname{str}\left(\mathcal{y}_{x}\right) \rightarrow \operatorname{str}\left(\mathcal{Y}_{x^{\prime}}\right): y \mapsto y^{\prime}$ from Proposition 2.9. Then, given an isometric bijection $\mu:[\mathbf{n}] \stackrel{\sim}{\rightarrow} \operatorname{irr}\left(\mathcal{y}_{x}, y\right)$, there exists a unique pair $\left(I, \mu^{\prime}\right)$ consisting of a subset $I \subset[p]$, where $p=w(\mathbf{n})$, and an isometric bijection $\mu^{\prime}:\left[\mathbf{n}_{I}\right] \stackrel{\sim}{\rightarrow} \operatorname{irr}\left(y_{x^{\prime}}, y^{\prime}\right)$ for which the following diagram is commutative

$$
\begin{aligned}
& {[\mathbf{n}] \stackrel{\mu}{\stackrel{\mu}{\rightarrow}} \operatorname{irr}\left(y_{x}, y\right)} \\
& \begin{array}{cc}
\downarrow & \downarrow \\
{\left[\mathbf{n}_{I}\right] \stackrel{\mu^{\prime}}{\stackrel{\sim}{\rightarrow}} \operatorname{irr}\left(y_{x^{\prime}}, y^{\prime}\right)}
\end{array}
\end{aligned}
$$


Proof. We can shrink $y$ and assume that it is elementary and $y$ is the unique maximal point of $\operatorname{str}(\mathcal{y})$. Then $y_{x}$ and $y_{x^{\prime}}$ are elementary, and $y$ and $y^{\prime}$ are their unique maximal points, respectively. The situation is then reduced to the case $\mathcal{X}=\operatorname{Spec}(A)$ and $y=\operatorname{Spec}(B)$, where $B=$ $B_{1} \otimes_{A} \cdots \otimes_{A} B_{p} \otimes_{A} C$ with $B_{i}=A\left[T_{i 0}, \ldots, T_{i n_{i}}\right] /\left(T_{i 0} \cdot \ldots \cdot T_{i n_{i}}-a_{i}\right)$ and $C=A\left[T_{1}, \ldots, T_{m}, \frac{1}{T_{1}}, \ldots, \frac{1}{T_{m}}\right]$, so that the isometric bijection $\mu$ is the evident one. In particular, $a_{i}(x)=0$ for all $i \in[p]$. We set $I=\{i \in$ $\left.[p] \mid a_{i}\left(x^{\prime}\right)=0\right\}$. Then $\operatorname{irr}\left(y_{x^{\prime}}, y^{\prime}\right)$ is evidently identified with $\left[\mathbf{n}_{I}\right]$ so that the diagram considered is commutative. That the pair $\left(I, \mu^{\prime}\right)$ is unique is already obvious.

Corollary 6.2. In the situation of Lemma 6.1, there is a canonical morphism of polysimplicial sets $\mathbf{C}\left(\mathcal{y}_{x}\right) \rightarrow \mathbf{C}\left(\mathcal{y}_{x^{\prime}}\right)$. If $x^{\prime \prime} \in \operatorname{str}(\mathcal{X})$ is a point with $x^{\prime \prime} \leq x^{\prime}$, then the morphism $\mathbf{C}\left(y_{x}\right) \rightarrow \mathbf{C}\left(y_{x^{\prime \prime}}\right)$ coincides with the composition of $\mathbf{C}\left(y_{x}\right) \rightarrow \mathbf{C}\left(y_{x^{\prime}}\right)$ and $\mathbf{C}\left(y_{x^{\prime}}\right) \rightarrow \mathbf{C}\left(y_{x^{\prime \prime}}\right)$.

Consider the following general construction. Let $C$ be a polysimplicial set, and let $D$ be a contravariant functor from the category $\Lambda / C$ to $\Lambda^{\circ} \mathcal{E} n s$ : $\left(\boldsymbol{\Lambda}[\mathbf{n}] \stackrel{\alpha_{x}}{\rightarrow} C\right) \mapsto D_{x}$. We define as follows a polysimplicial set

$$
C \square D=\operatorname{Coker}\left(\coprod \boldsymbol{\Lambda}\left[\mathbf{n}_{y}\right] \square D_{x} \rightarrow \coprod \boldsymbol{\Lambda}\left[\mathbf{n}_{x}\right] \square D_{x}\right)
$$

where the second coproduct is taken over the set of polysimplices of $C$, which can be identified with the set $N_{0}(\boldsymbol{\Lambda} / C)$ consisting of the morphisms $\boldsymbol{\Lambda}\left[\mathbf{n}_{x}\right] \stackrel{\alpha_{x}}{\rightarrow} C$, the first coproduct is taken over the set $N_{1}(\boldsymbol{\Lambda} / C)$ consisting of the commutative diagrams (with $y=C(f) x$ )

$$
\boldsymbol{\Lambda}\left[\mathbf{n}_{y}\right] \underset{\alpha_{y} \searrow}{\searrow} \stackrel{f}{\swarrow}_{C} \alpha_{x} \boldsymbol{\Lambda}\left[\mathbf{n}_{x}\right]
$$

the upper map $d_{0}$ takes $\boldsymbol{\Lambda}\left[\mathbf{n}_{y}\right] \square D_{x}$ to $\boldsymbol{\Lambda}\left[\mathbf{n}_{x}\right] \square D_{x}$ and acts as $f \square 1_{D_{x}}$, and the lower map $d_{1}$ takes $\Lambda\left[\mathbf{n}_{y}\right] \mathrm{a} D_{x}$ to $\Lambda\left[\mathbf{n}_{y}\right] \mathrm{\square} D_{y}$ and acts as $1_{\Lambda[\mathbf{n} y} \square D(f)$, where $D(f): D_{x} \rightarrow D_{y}$. For example, if $D$ is a constant functor $\left(\boldsymbol{\Lambda}\left[\mathbf{n}_{x}\right] \stackrel{\alpha_{x}}{\rightarrow} C\right)$ $\mapsto C^{\prime}$, where $C^{\prime} \in \mathrm{Ob}\left(\Lambda^{\circ} \mathcal{E} n s\right)$, then $C \square D$ is the usual square product $C \square C^{\prime}$. Thus, we get a functor $(\boldsymbol{\Lambda} / C)^{\circ}\left(\boldsymbol{\Lambda}^{\circ} \mathcal{E} n s\right) \rightarrow \Lambda^{\circ} \mathcal{E} n s: D \mapsto C \square D$ that commutes with direct limits. The construction is functorial on $C$, i.e., given a morphism of polysimplicial sets $C^{\prime} \rightarrow C$ and a morphism $D^{\prime} \rightarrow D$ over $C^{\prime} \rightarrow C$, there is a canonical morphism $C^{\prime} \square D^{\prime} \rightarrow C \square D$. We remark that the canonical morphisms $\Lambda\left[\mathbf{n}_{x}\right] \square D_{x} \rightarrow \Lambda\left[\mathbf{n}_{x}\right]$ give rise to a functorial morphism of polysimplicial sets $C \square D \rightarrow C$. We also remark that one can associate with each pair $\left(x^{\prime}, x^{\prime \prime}\right)$, consisting of polysimplices $x^{\prime} \in C_{\mathbf{n}^{\prime}}$ and $x^{\prime \prime} \in D_{x^{\prime}, \mathbf{n}^{\prime \prime}}$, a polysimplex $\left\langle x^{\prime}, x^{\prime \prime}\right\rangle \in(C \square D)_{\mathbf{n}}$, where $[\mathbf{n}]=\left[\mathbf{n}^{\prime}\right] \square\left[\mathbf{n}^{\prime \prime}\right]$. Namely, this pair defines a polysimplex $\left\langle e_{\mathbf{n}^{\prime}}, x^{\prime \prime}\right\rangle \in\left(\Lambda\left[\mathbf{n}^{\prime}\right] \square D_{x^{\prime}}\right)_{\mathbf{n}}$ (see $\left.\S 3\right)$, and $\left\langle x^{\prime}, x^{\prime \prime}\right\rangle$ is the image of the latter in $(C \square D)_{\mathbf{n}}$. (Recall that $e_{\mathbf{n}}$ denotes the fundamental $\mathbf{n}$-polysimplex of $\boldsymbol{\Lambda}[\mathbf{n}]$.) The following is a generalization of Lemma 3.6. 
Lemma 6.3. Assume that $D$ possesses the following property: for any surjective morphism $f:[\mathbf{m}] \rightarrow[\mathbf{n}]$ and any $x \in C_{\mathbf{n}}$, one has $D(f):$ $D_{x} \stackrel{\sim}{\rightarrow} D_{C(f) x}$. Then for each $x \in(C \square D)_{\mathbf{n}}$ there exists a unique tuple $\left(I^{\prime}, I^{\prime \prime}, x^{\prime}, x^{\prime \prime}\right)$ consisting of disjoint subsets $I^{\prime}, I^{\prime \prime} \subset[p], p=w(\mathbf{n})$, and polysimplices $x^{\prime} \in C_{\mathbf{n}_{I^{\prime}}}^{\text {nd }}$ and $x^{\prime \prime} \in D_{x^{\prime}, \mathbf{n}_{I^{\prime \prime}}}^{\text {nd }}$ such that $x=(C \square D)\left(\pi_{I^{\prime}, I^{\prime \prime}}\right)\left\langle x^{\prime}, x^{\prime \prime}\right\rangle$.

Proof. Existence. First, $x$ comes from a polysimplex $y \in\left(\Lambda[\mathbf{m}] \square D_{z^{\prime}}\right)_{\mathbf{n}}$ for some $z^{\prime} \in C_{\mathrm{m}}$. By Lemma 3.2, we can find disjoint subsets $J^{\prime}, J^{\prime \prime} \subset[p]$ and polysimplices $y^{\prime} \in \boldsymbol{\Lambda}[\mathbf{m}]_{\mathbf{n}_{J^{\prime}}}$ and $y^{\prime \prime} \in D_{z^{\prime}, \mathbf{n}_{J^{\prime \prime}}}$ with $y=\left(\boldsymbol{\Lambda}[\mathbf{m}] \square D_{z^{\prime}}\right)\left(\pi_{J^{\prime}, J^{\prime \prime}}\right)\left\langle y^{\prime}, y^{\prime \prime}\right\rangle$. If $g$ denotes the morphism $\boldsymbol{\Lambda}\left[\mathbf{n}_{J^{\prime}}\right] \rightarrow \boldsymbol{\Lambda}[\mathbf{m}]$ that corresponds to $y^{\prime}$, then $d_{0}\left\langle e_{\mathbf{n}_{I^{\prime}}}, y^{\prime \prime}\right\rangle=\left\langle y^{\prime}, y^{\prime \prime}\right\rangle$ and $d_{1}\left\langle e_{\mathbf{n}_{I^{\prime}}}, y^{\prime \prime}\right\rangle=\left\langle e_{\mathbf{n}_{I^{\prime}}}, D(g)\left(y^{\prime \prime}\right)\right\rangle$, where $D(g)$ is the morphism $D_{z^{\prime}} \rightarrow D_{C(g) z^{\prime}}$. Thus, we may assume that

$$
y=\left(\Lambda\left[\mathbf{n}_{J^{\prime}}\right] \square D_{z^{\prime}}\right)\left(\pi_{J^{\prime}, J^{\prime \prime}}\right)\left\langle e_{\mathbf{n}_{J^{\prime}}}, y^{\prime \prime}\right\rangle
$$

for some $z^{\prime} \in C_{\mathbf{n}_{J^{\prime}}}$ and $y^{\prime \prime} \in D_{z^{\prime}, \mathbf{n}_{J^{\prime \prime}}}$ and, therefore, $x=(C \square D)\left(\pi_{J^{\prime}, J^{\prime \prime}}\right)\left\langle z^{\prime}, y^{\prime \prime}\right\rangle$. Furthermore, let $z^{\prime}=C\left(\pi^{\prime}\right) x^{\prime}$, where $x^{\prime} \in C_{\mathbf{n}_{I^{\prime}}}^{\text {nd }}, I^{\prime}$ is a subset of $J^{\prime}$, and $\pi^{\prime}$ is the canonical surjective morphism $\left[\mathbf{n}_{J^{\prime}}\right] \rightarrow\left[\mathbf{n}_{I^{\prime}}\right]$. By the assumption, $D\left(\pi^{\prime}\right)$ : $D_{x^{\prime}} \stackrel{\sim}{\rightarrow} D_{z^{\prime}}$. Let $z^{\prime \prime}$ be the element of $D_{x^{\prime}, \mathbf{n}_{J^{\prime \prime}}}$ that goes to $y^{\prime \prime}$ under $D\left(\pi^{\prime}\right)$. One has $d_{1}\left\langle e_{\mathbf{n}_{I^{\prime}}}, z^{\prime \prime}\right\rangle=\left\langle e_{\mathbf{n}_{J^{\prime}}}, y^{\prime \prime}\right\rangle$ and $d_{0}\left\langle e_{\mathbf{n}_{I^{\prime}}}, z^{\prime \prime}\right\rangle=\left\langle y^{\prime}, z^{\prime \prime}\right\rangle$, where $y^{\prime}$ is the polysimplex from $\Lambda\left[\mathbf{n}_{I^{\prime}}\right]_{\mathbf{n}_{J^{\prime}}}$ that corresponds to the morphism $\pi^{\prime}$. Thus, we can replace $y$ by the element $\left(\boldsymbol{\Lambda}\left[\mathbf{n}_{I^{\prime}}\right] \square D_{x^{\prime}}\right)\left(\pi_{J^{\prime}, J^{\prime \prime}}\right)\left\langle y^{\prime}, z^{\prime \prime}\right\rangle$. The latter is equal to $\left(\boldsymbol{\Lambda}\left[\mathbf{n}_{I^{\prime}}\right] \square D_{x^{\prime}}\right)\left(\pi_{I^{\prime}, J^{\prime \prime}}\right)\left\langle e_{\mathbf{n}_{I^{\prime}}}, z^{\prime \prime}\right\rangle$ and, therefore, $x=(C \square D)\left(\pi_{I^{\prime}, J^{\prime \prime}}\right)\left\langle x^{\prime}, z^{\prime \prime}\right\rangle$. Finally, let $z^{\prime \prime}=D_{x^{\prime}}\left(\pi^{\prime \prime}\right) x^{\prime \prime}$, where $x^{\prime \prime} \in D_{x^{\prime}, \mathbf{n}_{I^{\prime \prime}}^{\text {nd }}}^{\text {nd }}, I^{\prime \prime}$ is a subset of $J^{\prime \prime}$, and $\pi^{\prime \prime}$ is the canonical morphism $\left[\mathbf{n}_{J^{\prime \prime}}\right] \rightarrow\left[\mathbf{n}_{I^{\prime \prime}}\right]$. Then $x=(C \square D)\left(\pi_{I^{\prime}, I^{\prime \prime}}\right)\left\langle x^{\prime}, x^{\prime \prime}\right\rangle$.

Uniqueness. 1) $I^{\prime}$ and $x^{\prime}$ are uniquely determined by $x$. Indeed, let $\varphi$ denote the canonical morphism $C \square D \rightarrow C$. Then $\varphi_{\mathbf{n}}(x)=C\left(\pi_{I^{\prime}}\right) x^{\prime}$ and the claim follows from the Eilenberg-Zilber Lemma 3.2.

2) Given $y^{\prime} \in C_{\mathbf{m}^{\prime}}^{\text {nd }}$ and $y^{\prime \prime} \in D_{y^{\prime}, \mathbf{m}^{\prime \prime}}^{\text {nd }}$, if $\left\langle y^{\prime}, y^{\prime \prime}\right\rangle=\left\langle y^{\prime}, \tilde{y}^{\prime \prime}\right\rangle$ for some $\tilde{y}^{\prime \prime} \in$ $D_{y^{\prime}, \mathbf{m}^{\prime \prime}}$ then $\widetilde{y}^{\prime \prime}=y^{\prime \prime}$. Indeed, let $[\mathbf{m}]=\left[\mathbf{m}^{\prime}\right]\left[\left[\mathbf{m}^{\prime \prime}\right], q=w(\mathbf{m})\right.$, and $[q]=$ $J^{\prime} \coprod J^{\prime \prime}$ the corresponding partition. Then there exist morphisms $\Lambda\left[n_{x_{l+1}}\right]=$ $\boldsymbol{\Lambda}\left[\mathbf{m}^{\prime}\right] \stackrel{f_{l}}{\rightarrow} \boldsymbol{\Lambda}\left[n_{x_{l}}\right] \stackrel{f_{l-1}}{\rightarrow} \ldots \stackrel{f_{1}}{\rightarrow} \boldsymbol{\Lambda}\left[n_{x_{1}}\right]=\boldsymbol{\Lambda}\left[\mathbf{m}^{\prime}\right]$ and polysimplices $z_{i} \in$ $\left(\Lambda\left[\mathbf{n}_{x_{i+1}}\right] \square D_{x_{i}}\right)_{\mathbf{m}}, 1 \leq i \leq l$, with $d_{0} z_{1}=\left\langle e_{\mathbf{m}^{\prime}}, y^{\prime \prime}\right\rangle, d_{1} z_{i}=d_{0} z_{i+1}$ for $1 \leq i \leq l-1$, and $\bar{d}_{1} z_{l}=\left\langle e_{\mathbf{m}^{\prime}}, \widetilde{y}^{\prime \prime}\right\rangle$. By Lemma 3.6, for each $1 \leq i \leq l$ there exists a unique tuple $\left(J_{i}^{\prime}, J_{i}^{\prime \prime}, \gamma_{i}, \delta_{i}\right)$ consisting of disjoint subsets $J_{i}^{\prime}, J_{i}^{\prime \prime} \subset[q]$ and polysimplices $\gamma_{i} \in \boldsymbol{\Lambda}\left[\mathbf{n}_{x_{i+1}}\right]_{\mathbf{m}_{J_{i}^{\prime}}}^{\text {nd }}$ and $\delta_{i} \in D_{x_{i}, \mathbf{m}_{J_{i}^{\prime \prime}}}^{\text {nd }}$ with $z_{i}=\left(\boldsymbol{\Lambda}\left[\mathbf{n}_{x_{i+1}}\right] \square D_{x_{i}}\right)\left(\pi_{J_{i}^{\prime}, J_{i}^{\prime \prime}}\right)\left\langle\gamma_{i}, \delta_{i}\right\rangle$. The uniqueness of Lemma 3.6 applied to the above equalities implies that $J_{i}^{\prime}=J^{\prime}$ and $J_{i}^{\prime \prime}=J^{\prime \prime}$ for all $1 \leq i \leq l$, $e_{\mathbf{m}^{\prime}}=\left(f_{1} \circ \cdots \circ f_{l}\right)\left(e_{\mathbf{m}^{\prime}}\right)$ and $\tilde{y}^{\prime \prime}=D\left(f_{1} \circ \cdots \circ f_{l}\right) y^{\prime \prime}$. The first of the latter two equalities implies that $f_{1} \circ \cdots \circ f_{l}=1_{\Lambda\left[\mathrm{m}^{\prime}\right]}$ and, therefore, $\widetilde{y}^{\prime \prime}=y^{\prime \prime}$.

$3)$ In the situation of 2) the polysimplex $\left\langle y^{\prime}, y^{\prime \prime}\right\rangle$ is nondegenerate. Indeed, let $\left\langle y^{\prime}, y^{\prime \prime}\right\rangle=(C a D)\left(\pi_{I}\right) z$ for some subset $I \subset[q]$ and a polysimplex $z \in(C \square D)_{\mathbf{m}_{I}}^{\text {nd }}$. By the existence, one has $z=(C \square D)_{\mathbf{m}_{I^{\prime}, I^{\prime \prime}}}\left\langle z^{\prime}, z^{\prime \prime}\right\rangle$ for some partition $I=I^{\prime} \bigsqcup I^{\prime \prime}$ and polysimplices $z^{\prime} \in C_{\mathbf{m}_{I^{\prime}, I^{\prime \prime}}}$ and $z^{\prime \prime} \in D_{z^{\prime}, \mathbf{m}_{I^{\prime \prime}}}$. We 
get $\left\langle y^{\prime}, y^{\prime \prime}\right\rangle=(C \square D)\left(\pi_{I^{\prime}, I^{\prime \prime}}\right)\left\langle z^{\prime}, z^{\prime \prime}\right\rangle$. By 1$), I^{\prime}=J^{\prime}$ and $z^{\prime}=y^{\prime}$ and, in particular, $I^{\prime \prime} \subset J^{\prime \prime}$. If $\pi^{\prime \prime}$ denotes the canonical morphism $\left[\mathbf{m}_{J^{\prime \prime}}\right] \rightarrow\left[\mathbf{m}_{I^{\prime \prime}}\right]$, then $\left\langle y^{\prime}, y^{\prime \prime}\right\rangle=\left\langle y^{\prime}, D_{y^{\prime}}\left(\pi^{\prime \prime}\right) z^{\prime \prime}\right\rangle$. From 2) it follows that $I^{\prime \prime}=J^{\prime \prime}$ and $z^{\prime \prime}=y^{\prime \prime}$.

4) $I^{\prime \prime}$ and $x^{\prime \prime}$ are uniquely determined by $x$. Indeed, since $\left\langle x^{\prime}, x^{\prime \prime}\right\rangle$ is nondegenerate, the Eilenberg-Zilber Lemma 3.2 implies that $I^{\prime} \cup I^{\prime \prime}$ and $\left\langle x^{\prime}, x^{\prime \prime}\right\rangle$ are uniquely determined by $x$. From 1) and 2) it follows that $I^{\prime \prime}$ and $x^{\prime \prime}$ are uniquely determined by $x$.

Corollary 6.4. In the situation of Lemma 6.3, given an injective morphism of polysimplicial sets $C^{\prime} \rightarrow C$ and an injective morphism $D^{\prime} \rightarrow D$ over $C^{\prime} \rightarrow C$ with $D^{\prime}$ also satisfying the assumption of Lemma 6.3, the induced morphism $C^{\prime} \square D^{\prime} \rightarrow C \square D$ is injective.

If $\sigma$ is an automorphism of $[\mathbf{n}]$ associated with a triple $([p], f, \alpha)$ then, for any subset $I \subset[p]$, the triple $\left(f^{-1}(I),\left.f\right|_{f^{-1}(I)}, \alpha_{f^{-1}(I)}\right)$ defines an isomorphism $\sigma_{I}:\left[\mathbf{n}_{f^{-1}(I)}\right] \stackrel{\sim}{\rightarrow}\left[\mathbf{n}_{I}\right]$. One has $\pi_{I} \circ \sigma=\sigma_{I} \circ \pi_{f^{-1}(I)}$. It is easy to check that for $x=(C a D)\left(\pi_{I^{\prime}, I^{\prime \prime}}\right)\left\langle x^{\prime}, x^{\prime \prime}\right\rangle$ one has

$$
(C \square D)(\sigma) x=(C \square D)\left(\pi_{f^{-1}\left(I^{\prime}\right), f^{-1}\left(I^{\prime \prime}\right)}\right)\left\langle C\left(\sigma_{I^{\prime}}\right) x^{\prime}, D\left(\sigma_{I^{\prime}}\right)\left(D_{x^{\prime}}\left(\sigma_{I^{\prime \prime}}\right) x^{\prime \prime}\right)\right\rangle .
$$

Corollary 6.5. In the situation of Lemma 6.3, let $x^{\prime} \in C_{\mathbf{n}^{\prime}}^{\text {nd }}, x^{\prime \prime} \in D_{x^{\prime}, \mathbf{n}^{\prime \prime}}^{\text {nd }}$ and $[\mathbf{n}]=\left[\mathbf{n}^{\prime}\right] \square\left[\mathbf{n}^{\prime \prime}\right]$. Then $\left\langle x^{\prime}, x^{\prime \prime}\right\rangle \in(C \square D)_{\mathbf{n}}^{\text {nd }}$ and $G_{\left\langle x^{\prime}, x^{\prime \prime}\right\rangle}=\left\{\left(g^{\prime}, g^{\prime \prime}\right) \in\right.$ $\left.G_{x^{\prime}} \times \operatorname{Aut}\left(\left[\mathbf{n}^{\prime \prime}\right]\right) \mid x^{\prime \prime}=D\left(g^{\prime}\right)\left(g^{\prime \prime} x^{\prime \prime}\right)\right\}$. In particular, if $C$ and all $D_{x}$ are interiorly free, then so is $C \square D$.

Corollary 6.6. In the situation of Lemma 6.3, assume that for a polysimplex $x \in C_{\mathbf{n}}^{\mathrm{nd}}$ the group $G_{x}$ acts trivially on the polysimplicial set $D_{x}$. Then the preimage of the corresponding cell $G_{x} \backslash \stackrel{\circ}{\Sigma}^{\mathbf{n}} \subset|C|$ in $|C \square D|$ is canonically homeomorphic to $\left(G_{x} \backslash \stackrel{\circ}{\Sigma}^{\mathbf{n}}\right) \times\left|D_{x}\right|$.

Assume we are given a strictly pluri-nodal scheme $\mathcal{X}$ and a polysimplicial set $E$ provided with a map of partially ordered sets $O(E) \rightarrow \operatorname{str}(\mathcal{X})$ : $(\boldsymbol{\Lambda}[\mathbf{n}] \stackrel{\alpha}{\rightarrow} E) \mapsto \bar{\alpha}$. Then every strictly poly-stable morphism $\varphi: \mathcal{Y} \rightarrow \mathcal{X}$ gives rise to a contravariant functor $D_{\varphi}: \Lambda / E \rightarrow \Lambda^{\circ} \mathcal{E} n s$ defined by $D_{\varphi}(\boldsymbol{\Lambda}[\mathbf{n}] \stackrel{\alpha}{\rightarrow} E)=\mathbf{C}\left(y_{\bar{\alpha}}\right)$. Note that $D_{\varphi}$ satisfies the assumptions of Lemma 6.3 and Corollary 6.6. We set $E_{\varphi}=E \square D_{\varphi}$. By Lemma 3.3 and Proposition 3.14(ii), one has

$$
O\left(E_{\varphi}\right)=\operatorname{Coker}\left(\coprod O(\boldsymbol{\Lambda}[\mathbf{m}]) \times \operatorname{str}\left(y_{\bar{\alpha}}\right) \stackrel{\rightarrow}{\rightarrow} \coprod O(\boldsymbol{\Lambda}[\mathbf{n}]) \times \operatorname{str}\left(y_{\bar{\alpha}}\right)\right),
$$

where the first and the second coproducts are taken over the categories $N_{1}(\boldsymbol{\Lambda} / E)$ and $N_{0}(\boldsymbol{\Lambda} / E)$, respectively. The map $d_{0}$ takes a pair $\left.\widetilde{\delta}, y\right)$, consisting of the class $\delta$ of a morphism $\delta: \Lambda[\mathbf{r}] \rightarrow \Lambda[\mathbf{m}]$ and a point $y \in \operatorname{str}\left(y_{\bar{\alpha}}\right)$, to the pair $(\widetilde{f \circ \delta}, y)$, where $f$ is the morphism $\boldsymbol{\Lambda}[\mathbf{m}] \rightarrow \boldsymbol{\Lambda}[\mathbf{n}]$, and the map $d_{1}$ takes the same pair $\left.\widetilde{\delta}, y\right)$ to the pair $\left(\widetilde{\delta}, y^{\prime}\right)$, where $y^{\prime}$ is the image of $y$ under map $\operatorname{str}\left(\mathcal{y}_{\bar{\alpha}}\right) \rightarrow \operatorname{str}\left(\mathcal{y}_{\overline{\alpha \circ f}}\right)$. It follows that the maps 
$O(\boldsymbol{\Lambda}[\mathbf{n}]) \times \operatorname{str}\left(\mathcal{y}_{\bar{\alpha}}\right) \rightarrow \operatorname{str}\left(\mathcal{y}_{)}\right)$, that take a pair $(\bar{\delta}, y)$ to the image of the point $y$ under the map $\operatorname{str}\left(y_{\bar{\alpha}}\right) \rightarrow \operatorname{str}\left(\mathcal{Y}_{\overline{\alpha \circ \delta}}\right)$, define a map of partially ordered sets $O\left(E_{\varphi}\right) \rightarrow \operatorname{str}(y)$.

Lemma 6.7. In the above situation the following is true:

(i) if $O(E) \stackrel{\sim}{\rightarrow} \operatorname{str}(\mathcal{X})$, then $O\left(E_{\varphi}\right) \stackrel{\sim}{\rightarrow} \operatorname{str}(\mathcal{Y})$;

(ii) if $E$ is interiorly free, then so is $E_{\varphi}$;

(iii) given an element $\alpha \in O(E)$, the preimage of the corresponding cell of $|E|$ in $\left|E_{\varphi}\right|$ is canonically homeomorphic to a direct product of it with $\left|\mathbf{C}\left(y_{\bar{\alpha}}\right)\right|$.

Proof. (i) follows from Lemma 6.3 and Proposition 2.7(ii), (ii) follows from Corollary 6.5, and (iii) follows from Corollary 6.6.

We now associate with a strictly poly-stable fibration $\underline{X}=\left(X_{l} \stackrel{f_{l-1}}{\rightarrow}\right.$ $\left.\ldots \stackrel{f_{1}}{\rightarrow} X_{1} \stackrel{f_{0}}{\rightarrow} X_{0}=\operatorname{Spec}(K)\right)$ over a field $K$ a polysimplicial set $\mathbf{C}(\underline{\mathcal{X}})$ as follows. If $l=1$ then $\mathbf{C}(\underline{\mathcal{X}})=\mathbf{C}\left(\mathcal{X}_{1}\right)$, and if $l \geq 2$ then $\mathbf{C}(\underline{\mathcal{X}})=$ $\mathbf{C}\left(\underline{\mathcal{X}}^{\prime}\right)_{f_{l-1}}$, where $\underline{\mathcal{X}}^{\prime}=\left(\mathcal{X}_{l-1} \stackrel{f_{l-2}}{\rightarrow} \cdots \stackrel{f_{1}}{\rightarrow} \mathcal{X}_{1}\right)$. (We omit $\mathcal{X}_{0}=\operatorname{Spec}(K)$ if its presence is evident.) By Corollary 6.4, the polysimplicial set $\mathbf{C}(\underline{X})$ is interiorly free, and there is a canonical isomorphism of partially ordered sets $O(\mathbf{C}(\underline{\mathcal{X}})) \stackrel{\sim}{\rightarrow} \operatorname{str}\left(\mathcal{X}_{l}\right)$. The construction is obviously functorial, and we get a functor $\mathbf{C}^{l}$ from the full subcategory of $\mathcal{P} s t_{l}^{\mathrm{tps}}$ consisting of strictly poly-stable fibrations to $\Lambda^{\circ} \mathcal{E} n s$.

Using Lemma 6.3, one can describe the sets $\mathbf{C}_{\mathbf{n}}(\underline{\mathcal{X}})$ as follows. For each $\mathbf{n}$ with $p=w(\mathbf{n})$, there is a canonical bijection between $\mathbf{C}_{\mathbf{n}}(\underline{\mathcal{X}})$ and the set of the triples $(x, \underline{I}, \mu)$, where $x \in \operatorname{str}\left(\mathcal{X}_{l}\right), \underline{I}=\left(I_{1}, \ldots, I_{l}\right)$ is a family of pairwise disjoint subsets of [ $p$ ] (all $I_{k}$ are assumed to be empty if $[\mathbf{n}]=[0])$, and $\underline{\mu}=\left(\mu_{1}, \ldots, \mu_{l}\right)$ is a family of isometric bijections $\mu_{k}:\left[\mathbf{n}_{I_{k}}\right] \stackrel{\sim}{\rightarrow} \operatorname{irr}\left(\mathcal{X}_{k, x_{k-1}}, x_{k}\right)$, where $x_{k}$ is the image of the point $x$ in $X_{k}$. Given a morphism $\gamma:\left[\mathbf{n}^{\prime}\right] \rightarrow[\mathbf{n}]$ associated with a triple $(J, f, \alpha)$, the map $\mathbf{C}_{\mathbf{n}}(\underline{\mathcal{X}}) \rightarrow \mathbf{C}_{\mathbf{n}^{\prime}}(\underline{\mathcal{X}})$ takes a triple $(x, \underline{I}, \underline{\mu})$ to the triple $\left(x^{\prime}, \underline{I}^{\prime}, \mu^{\prime}\right)$ defined inductively as follows. One has $I_{1}^{\prime}=f^{-1}\left(I_{1}\right)$, and the set $I_{1}^{\prime}$ defines a point $x_{1}^{\prime} \in X_{1}$ with $x_{1}^{\prime} \leq x_{1}$ and an isometric bijection $\mu_{1}^{\prime}:\left[\mathbf{n}_{I_{1}^{\prime}}^{\prime}\right] \stackrel{\sim}{\rightarrow} \operatorname{irr}\left(\mathcal{X}_{1}, x_{1}^{\prime}\right)$ for which the following diagram is commutative

$$
\begin{aligned}
& {\left[\mathbf{n}_{I_{1}}\right] \stackrel{\mu_{1}}{\sim} \operatorname{irr}\left(\mathcal{X}_{1}, x_{1}\right)} \\
& \stackrel{\uparrow}{\stackrel{\mu_{1}^{\prime}}{\stackrel{\uparrow}{\sim}} \operatorname{irr}\left(\mathcal{X}_{1}, x_{1}^{\prime}\right)}
\end{aligned}
$$

Assume that $2 \leq k \leq l$ and that the points $x_{j}^{\prime}$ with $x_{j}^{\prime} \leq x_{j}$, the sets $I_{j}^{\prime}$ and the isometric bijections $\mu_{j}^{\prime}:\left[\mathbf{n}_{I_{j}^{\prime}}^{\prime} \stackrel{\sim}{\rightarrow} \operatorname{irr}\left(\mathcal{X}_{j, x_{j-1}^{\prime}}, x_{j}^{\prime}\right)\right.$ are constructed for 
$1 \leq j \leq k-1$. First, the set $J^{\prime}=f^{-1}\left(I_{k}\right)$ defines a point $y^{\prime} \in X_{k, x_{k-1}}$ with $y^{\prime} \leq x_{k}$ and an isometric bijection $v^{\prime}:\left[\mathbf{n}_{J^{\prime}}^{\prime}\right] \stackrel{\sim}{\rightarrow} \operatorname{irr}\left(\mathcal{X}_{k, x_{k-1}}, y^{\prime}\right)$ for which the following diagram is commutative

$$
\begin{gathered}
{\left[\mathbf{n}_{I_{k}}\right] \stackrel{\stackrel{\mu_{k}}{\rightarrow}}{\rightarrow} \operatorname{irr}\left(\mathcal{X}_{k, x_{k-1}}, x_{k}\right)} \\
\uparrow \\
{\left[\mathbf{n}_{J^{\prime}}^{\prime} \stackrel{\stackrel{\nu^{\prime}}{\rightarrow}}{\rightarrow} \operatorname{irr}\left(\mathcal{X}_{k, x_{k-1}}, y^{\prime}\right)\right.}
\end{gathered}
$$

Let $x_{k}^{\prime}$ be the image of the point $y^{\prime}$ under the canonical map $\operatorname{str}\left(\mathcal{X}_{k, x_{k-1}}\right) \rightarrow$ $\operatorname{str}\left(\mathcal{X}_{k, x_{k-1}^{\prime}}\right)$. By Lemma 6.1, there exists a unique pair $\left(I_{k}^{\prime}, \mu_{k}^{\prime}\right)$ consisting of a subset $I_{k}^{\prime} \subset J^{\prime}$ and an isometric bijection $\mu_{k}^{\prime}:\left[\mathbf{n}_{I_{k}^{\prime}}^{\prime}\right] \stackrel{\sim}{\rightarrow} \operatorname{irr}\left(\mathcal{X}_{k, x_{k-1}^{\prime}}, x_{k}^{\prime}\right)$ for which the following diagram is commutative

$$
\begin{gathered}
{\left[\mathbf{n}_{J^{\prime}}^{\prime}\right] \stackrel{\stackrel{\nu^{\prime}}{\sim}}{\rightarrow} \operatorname{irr}\left(\mathcal{X}_{k, x_{k-1}}, y^{\prime}\right)} \\
\downarrow \\
\downarrow \\
{\left[\mathbf{n}_{I_{k}^{\prime}}^{\prime}\right] \stackrel{\mu_{k}^{\prime}}{\stackrel{\sim}{\rightarrow}} \operatorname{irr}\left(\mathcal{X}_{k, x_{k-1}^{\prime}}, x_{k}^{\prime}\right)}
\end{gathered}
$$

Setting $x^{\prime}=x_{l}^{\prime}, \underline{I}^{\prime}=\left(I_{1}^{\prime}, \ldots, I_{l}^{\prime}\right)$ and $\underline{\mu}^{\prime}=\left(\mu_{1}^{\prime}, \ldots, \mu_{l}^{\prime}\right)$, we get the required triple $\left(x^{\prime}, \underline{I}^{\prime}, \underline{\mu}^{\prime}\right)$. Given a trivially poly-stable morphism $\varphi: \underline{y} \rightarrow \underline{\underline{X}}$, the map $\mathbf{C}_{\mathbf{n}}(\underline{\mathcal{Y}}) \rightarrow \mathbf{C}_{\mathbf{n}}(\underline{\mathcal{X}})$ takes a triple $(y, \underline{J}, \underline{v})$ to the triple $(x, \underline{I}, \underline{\mu})$, where $x=\varphi_{l}(y), I_{k}$ is the subset of $J_{k}$, and $\mu_{k}:\left[\mathbf{n}_{I_{k}}\right] \stackrel{\sim}{\rightarrow} \operatorname{irr}\left(\mathcal{X}_{k, x_{k-1}}, x_{k}\right)$ is the isometric bijection for which the following diagram is commutative

$$
\begin{gathered}
{\left[\mathbf{n}_{J_{k}}\right] \stackrel{\stackrel{\nu_{k}}{\rightarrow}}{\rightarrow} \operatorname{irr}\left(\mathcal{Y}_{k, y_{k-1}}, y_{k}\right)} \\
\downarrow \\
\quad \downarrow \\
{\left[\mathbf{n}_{I_{k}}\right] \stackrel{\stackrel{\mu_{k}}{\rightarrow}}{\rightarrow} \operatorname{irr}\left(\mathcal{X}_{k, x_{k-1}}, x_{k}\right)}
\end{gathered}
$$

The above explicit description of the sets $\mathbf{C}_{\mathbf{n}}(\underline{\mathcal{X}})$ easily implies the following fact.

Lemma 6.8. Let $\varphi: \underline{\mathcal{X}}^{\prime} \rightarrow \underline{\mathcal{X}}$ be a morphism between strictly poly-stable fibrations in $\mathcal{P}$ st ${ }_{l}^{\mathrm{sm}}$, and assume that $\operatorname{str}\left(\mathcal{X}_{l}^{\prime}\right) \stackrel{\sim}{\rightarrow} \operatorname{str}\left(\mathcal{X}_{l}\right)$. Then $\mathbf{C}\left(\underline{\mathcal{X}}^{\prime}\right) \stackrel{\sim}{\rightarrow} \mathbf{C}(\underline{\mathcal{X}})$.

Proposition 6.9. (i) The above functor $\underline{\underline{X}} \mapsto \mathbf{C}(\underline{\mathcal{X}})$ extends in a way, unique up to a unique isomorphism, to a functor $\mathbf{C}^{l}: \mathcal{P}_{s t_{l}}^{\text {tps }} \rightarrow \Lambda^{\circ}$ Ens so that, 
for any surjective étale morphism $\underline{\mathcal{X}^{\prime}} \rightarrow \underline{\mathcal{X}}$, one has $\operatorname{Coker}\left(\mathbf{C}\left(\underline{\mathcal{X}}^{\prime} \times \underline{x} \underline{\mathcal{X}^{\prime}}\right)\right.$ $\left.\rightarrow \mathbf{C}\left(\underline{\mathcal{X}}^{\prime}\right)\right) \stackrel{\sim}{\rightarrow} \mathbf{C}(\underline{\mathcal{X}})$;

(ii) for each poly-stable fibration $\underline{\mathcal{X}}$ there is a functorial isomorphism of partially ordered sets $O(\mathbf{C}(\underline{\mathcal{X}})) \stackrel{\sim}{\rightarrow} \operatorname{str}\left(\mathcal{X}_{l}\right)$;

(iii) given $\underline{X}=\left(X_{l} \stackrel{f_{l-1}}{\rightarrow} \cdots \stackrel{f_{1}}{\rightarrow} X_{1}\right)$ with $l \geq 2$, assume that for some $1 \leq k \leq l-1$ the morphism $f_{k}$ is trivially poly-stable; then $\mathbf{C}(\underline{\mathcal{X}}) \stackrel{\sim}{\rightarrow} \mathbf{C}\left(\underline{\mathcal{X}}^{\prime}\right)$, where $\underline{X}^{\prime}=\left(\cdots \rightarrow X_{k+1} \stackrel{f_{k-1} f_{k}}{\rightarrow} X_{k-1} \rightarrow \ldots\right)$; in particular, if all of the morphisms $f_{i}$ are trivially poly-stable, then $\mathbf{C}(\underline{\mathcal{X}})=\mathbf{C}\left(\mathcal{X}_{l}\right)$.

Proof. (i) As in our previous constructions, it suffices to verify the property stated for any surjective étale morphism of strictly poly-stable fibrations $\varphi: \underline{\mathcal{X}}^{\prime} \rightarrow \underline{\mathcal{X}}$. Let first $(x, \underline{I}, \underline{\mu})$ be from $\mathbf{C}_{\mathbf{n}}(\underline{\mathcal{X}})$, and take a point $x^{\prime} \in \operatorname{str}\left(\mathcal{X}_{l}^{\prime}\right)$ over $x$. Then there are isometric bijections $\gamma_{k}: \operatorname{irr}\left(\mathcal{X}_{k, x_{k-1}^{\prime}}, x_{k}^{\prime}\right) \stackrel{\sim}{\rightarrow} \operatorname{irr}\left(X_{k, x_{k-1}}, x_{k}\right)$, and the triple $(x, \underline{I}, \underline{\mu})$ is the image of the triple $\left(x^{\prime}, \underline{I}, \underline{\mu^{\prime}}\right)$ from $\mathbf{C}_{\mathbf{n}}\left(\underline{\mathcal{X}}^{\prime}\right)$, where $\mu_{k}^{\prime}=\mu_{k} \circ \gamma_{k}^{-1}$. Assume now that the images of two triples $\left(x^{\prime}, \underline{I}^{\prime}, \mu^{\prime}\right)$ and $\left(y^{\prime}, \underline{J}^{\prime}, \underline{v}^{\prime}\right)$ from $\mathbf{C}_{\mathbf{n}}\left(\underline{\mathcal{X}}^{\prime}\right)$ coincide in $\mathbf{C}_{\mathbf{n}}(\underline{\mathcal{X}})$ with a triple $(x, \underline{I}, \underline{\mu})$. We can find a point $x^{\prime \prime} \in \operatorname{str}\left(\mathcal{X}_{l}^{\prime \prime}\right)$, where $\underline{X}^{\prime \prime}=\underline{X}^{\prime} \times x \underline{X}^{\prime}$, whose two projections in $X_{l}^{\prime}$ are the points $x^{\prime}$ and $y^{\prime}$. It follows that the compositions of the isometric bijections $\operatorname{irr}\left(\mathcal{X}_{k, x_{k-1}^{\prime \prime}}^{\prime \prime}, x_{k}^{\prime \prime}\right) \stackrel{\gamma_{k}}{\rightarrow} \operatorname{irr}\left(\mathcal{X}_{k, x_{k-1}^{\prime}}^{\prime}, x_{k}^{\prime}\right) \stackrel{\sim}{\rightarrow} \operatorname{irr}\left(X_{k, x_{k-1}}, x_{k}\right)$ and $\operatorname{irr}\left(\mathcal{X}_{k, x_{k-1}^{\prime \prime}}^{\prime \prime}, x_{k}^{\prime \prime}\right) \stackrel{\delta_{k}}{\rightarrow} \operatorname{irr}\left(\mathcal{X}_{k, y_{k-1}^{\prime}}^{\prime}, y_{k}^{\prime}\right) \stackrel{\sim}{\rightarrow} \operatorname{irr}\left(\mathcal{X}_{k, x_{k-1}}, x_{k}\right)$ coincides. This implies that $I_{k}^{\prime}=I_{k}=J_{k}^{\prime}$ and that the isometric bijections $\mu_{k}^{\prime \prime}=\gamma^{-1} \circ \mu_{k}^{\prime}$ and $\delta^{-1} \circ v_{k}^{\prime}$ between $\left[\mathbf{n}_{I_{k}}\right]$ and $\operatorname{irr}\left(\mathcal{X}_{k, x_{k-1}^{\prime \prime}}^{\prime \prime}, x_{k}^{\prime \prime}\right)$ coincide. Thus the triple $\left(x^{\prime \prime}, \underline{I}, \underline{\mu}^{\prime \prime}\right)$ goes to the triples $\left(x^{\prime}, \underline{I}^{\prime}, \underline{\mu^{\prime}}\right)$ and $\left(y^{\prime}, \underline{J}^{\prime}, \underline{v}^{\prime}\right)$ under the two projections from $\underline{\mathcal{X}}^{\prime \prime}$ to $\underline{\underline{X}}^{\prime}$.

(ii) follows from (i), Lemma 3.3 and the fact that it is true for strictly poly-stable fibrations.

(iii) By (i), it suffices to verify the statement only for strictly poly-stable fibrations, but in this case it is easily deduced from the explicit description of elements of the sets $\mathbf{C}_{\mathbf{n}}(\underline{\underline{X}})$.

Corollary 6.10. Let $\underline{\underline{\chi}}$ be a poly-stable fibration of length $l$ over a field $K$. Then

(i) If $X_{l}$ is quasi-compact, then there exists a finite separable extension $K^{\prime}$ of $K$ such that, for any bigger field $K^{\prime \prime}$, one has $\mathbf{C}\left(\underline{\mathcal{X}} \otimes K^{\prime \prime}\right) \stackrel{\sim}{\rightarrow}$ $\mathbf{C}\left(\underline{\mathcal{X}} \otimes K^{\prime}\right)$.

(ii) If $\underline{\underline{X}}$ is a strictly poly-stable fibration and all of the strata of $\mathcal{X}_{l}$ are geometrically irreducible, then the conclusion of $(i)$ is true for $K^{\prime}=K$. 


\section{A lifting of a homotopy for a poly-stable morphism of formal schemes}

Let $\varphi: \mathfrak{Y} \rightarrow \mathfrak{X}$ be a poly-stable morphism between formal schemes locally finitely presented over $k^{\circ}$. For a point $x \in \mathfrak{X}_{\eta}$, we set $\mathfrak{Y}_{x}=$ $\mathfrak{Y} \times \mathfrak{X} \operatorname{Spf}\left(\mathscr{H}(x)^{\circ}\right)$. It is a poly-stable formal scheme over $\mathscr{H}(x)^{\circ}$, and there are canonical isomorphisms $\mathfrak{Y}_{x, \eta} \stackrel{\sim}{\rightarrow} \mathfrak{Y}_{\eta, x}$ and $\mathfrak{Y}_{x, s} \stackrel{\sim}{\rightarrow} \mathfrak{Y}_{s, \mathbf{x}} \otimes_{\widetilde{k}(\mathbf{x})} \widetilde{\mathscr{H}(x)}$, where $\mathbf{x}=\pi(x)$. Let $\Phi_{\mathfrak{X}}$ denote the mapping $\mathfrak{Y}_{\eta} \times[0,1] \rightarrow \mathfrak{Y}_{\eta}$ that coincides with the mapping $\Phi$ from Theorem 5.2 on each fibre of the morphism $\varphi_{\eta}$.

Theorem 7.1. The mapping $\Phi_{\mathfrak{X}}: \mathfrak{Y}_{\eta} \times[0,1] \rightarrow \mathfrak{Y}_{\eta}$ is continuous and proper. In particular, it is a strong deformation retraction of $\mathfrak{Y}_{\eta}$ to the closed set (the skeleton of the morphism $\varphi$ )

$$
S(\mathfrak{Y} / \mathfrak{X})=\cup_{x \in \mathfrak{X}_{\eta}} S\left(\mathfrak{Y}_{x}\right) .
$$

The natural question now is as follows. When a homotopy $\Psi: \mathfrak{X}_{\eta} \times$ $[0,1] \rightarrow \mathfrak{X}_{\eta}$ can be lifted to a homotopy $\widetilde{\Psi}: S(\mathfrak{Y} / \mathfrak{X}) \times[0,1] \rightarrow S(\mathfrak{Y} / \mathfrak{X})$ ? The following is an answer to the question in a situation which is sufficient for our purposes.

Assume we are given a formal scheme $\mathfrak{X}$ locally finitely presented over $k^{\circ}$ and, for each morphism $\mathfrak{X}^{\prime} \rightarrow \mathfrak{X}$ in $\mathcal{F} s c h^{\text {ét }}$, a homotopy $\Psi_{\mathfrak{X}^{\prime}}: \mathfrak{X}_{\eta}^{\prime} \times[0,1] \rightarrow$ $\mathfrak{X}_{\eta}^{\prime}:\left(x^{\prime}, t\right) \mapsto x_{t}^{\prime}$ with the properties

(a) $\left(x_{t}^{\prime}\right)_{t^{\prime}}=x_{\max \left(t, t^{\prime}\right)}^{\prime}$ and $x^{\prime} \leq x_{t}^{\prime}$ for all $x^{\prime} \in \mathfrak{X}_{\eta}^{\prime}$ and $0 \leq t, t^{\prime} \leq 1$;

(b) given a commutative diagram in $\mathcal{F} s c h^{\text {ét }}$

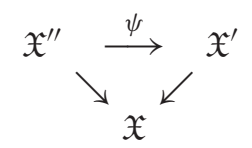

one has $\psi_{\eta}\left(x_{t}^{\prime \prime}\right)=\psi_{\eta}\left(x^{\prime \prime}\right)_{t}$ for all $x^{\prime \prime} \in \mathfrak{X}_{\eta}^{\prime \prime}$ and $0 \leq t \leq 1$.

Theorem 7.2. In the above situation, one can construct, for every polystable morphism $\varphi: \mathfrak{Y} \rightarrow \mathfrak{X}$, a proper homotopy $\widetilde{\Psi}: S(\mathfrak{Y} / \mathfrak{X}) \times[0,1] \rightarrow$ $S(\mathfrak{Y} / \mathfrak{X}):(y, t) \mapsto y_{t}$ over $\Psi=\Psi_{\mathfrak{X}}$ so that the following holds:

(i) $\left(y_{t}\right)_{t^{\prime}}=y_{\max \left(t, t^{\prime}\right)}$ and for all $0 \leq t, t^{\prime} \leq 1$;

(ii) $y \leq y_{t}$ for all $0 \leq t \leq 1$;

(iii) if $x=x_{t}\left(\right.$ resp. $\pi(x)=\pi\left(x_{t}\right)$ ) for some $0 \leq t \leq 1$ then $y=y_{t}$ (resp. $\left.\pi(y)=\pi\left(y_{t}\right)\right)$, where $x=\varphi_{\eta}(y)$;

(iv) given a commutative diagram

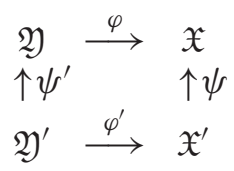

where $\varphi$ and $\varphi^{\prime}$ are poly-stable and $\psi$ and $\psi^{\prime}$ are morphisms in $\mathcal{F}$ sch ${ }^{\text {ét }}$, one has $\psi_{\eta}^{\prime}\left(y_{t}^{\prime}\right)=\psi_{\eta}^{\prime}\left(y^{\prime}\right)_{t}$ for all $y^{\prime} \in S\left(\mathfrak{Y}^{\prime} / \mathfrak{X}^{\prime}\right)$ and $0 \leq t \leq 1$. 
Notice that the properness in both theorems trivially follows from the continuity (see Step 9 from \$5).

We say that a strictly pluri-nodal scheme $\mathcal{X}$ over a field $K$ is geometrically elementary if it is elementary, i.e., the partially ordered set $\operatorname{str}(\mathcal{X})$ has a unique maximal element, and all of the strata of $\mathcal{X}$ are geometrically irreducible, i.e., for every bigger field $K^{\prime} \supset K$ one has $\operatorname{str}\left(\mathcal{X} \otimes K^{\prime}\right) \stackrel{\sim}{\rightarrow} \operatorname{str}(\mathcal{X})$. Furthermore, we say that a strictly pluri-nodal morphism $\varphi: \mathcal{y} \rightarrow \mathcal{X}$ is geometrically elementary if the fibre $y_{x}$ of $y$ at each point $x \in \mathcal{X}$ is geometrically elementary.

Lemma 7.3. Let $\varphi: \mathcal{Y} \rightarrow \mathcal{X}$ be a strictly poly-stable morphism between schemes of locally finite type over $K$. Then for each point $y \in \mathcal{y}$ there exist an étale morphism $X^{\prime} \rightarrow \mathcal{X}$ and an open subset $\mathcal{Y}^{\prime} \subset \mathcal{Y} \times X_{x} \mathcal{X}^{\prime}$ such that the image of $y^{\prime}$ in $y$ contains the point $y$ and the induced morphism $y^{\prime} \rightarrow X^{\prime}$ is geometrically elementary.

Proof. We may assume that the point $y$ is closed. Furthermore, to prove the statement we always can replace the ground field by a finite (not necessarily separable) extension of $K$ (see [SGA1, Exp. IX, 4.10]). In particular, we may assume that the point $y$ is $K$-rational. Shrinking $\mathcal{X}$ and $\mathcal{Y}$, we may assume that $\mathcal{X}=\operatorname{Spec}(A)$ is affine and the morphism $\varphi$ goes through an étale morphism $g: \mathcal{Y} \rightarrow \mathcal{Z}=\operatorname{Spec}(B)$ with $B=B_{0} \otimes_{A} \cdots \otimes_{A} B_{q}$, where $B_{i}=A\left[T_{i 0}, \ldots, T_{i n_{i}}\right] /\left(T_{i 0} \cdot \ldots \cdot T_{i n_{i}}-a_{i}\right), a_{i} \in A$ and $n_{i} \geq 1$. We may assume that $a_{i}(x)=0$ and $T_{i j}(y)=0$ for all $0 \leq i \leq p$ and $0 \leq j \leq n_{i}$, where $x$ is the image of $y$ in $\mathcal{X}$, and $a \in A^{*}$ for all $p+1 \leq i \leq q$. For a subset $I \subset[p]$, let $\mathcal{X}_{I}$ denote the set of the points of $\mathcal{X}$ where the functions $a_{i}$ with $i \in I$ vanish. (For example, $\mathcal{X}_{\emptyset}=\mathcal{X}_{\text {.) }}$ Given a subset $I \subset[p]$, assume that for every strictly bigger subset $I \subset J \subset[p]$ the fiber $\mathcal{y}_{x^{\prime}}$ at each point $x^{\prime} \in \mathcal{X}_{J}$ is geometrically elementary. We will prove the existence of $X^{\prime}$ and $y^{\prime}$, as in the lemma, with the property that the fibre $y_{x^{\prime}}^{\prime}$ of $y^{\prime}$ at each point $x^{\prime} \in \mathcal{X}^{\prime}$ over $\mathcal{X}_{I}$ is geometrically elementary.

Let $B_{I}$ (resp. $B_{I}^{\prime}$ ) denote the tensor product of $B_{i}$ 's for $i \in I$ (resp. $i \in[q] \backslash I)$. If $f$ denotes the canonical morphism $\mathcal{Z} \rightarrow \mathcal{X}$, then $f^{-1}\left(\mathcal{X}_{I}\right)=$ $\mathcal{U} \times x \mathcal{U}^{\prime}$, where $U=\operatorname{Spec}\left(B_{I}\right) \times x \mathcal{X}_{I}$ and $\mathcal{U}^{\prime}=\operatorname{Spec}\left(B_{I}^{\prime}\right) \times x_{x} \mathcal{X}_{I}$. One has $\mathcal{V}:=\left\{z \in \mathcal{U} \mid T_{i j}(z)=0\right.$ for all $i \in I$ and $\left.0 \leq j \leq n_{i}\right\} \stackrel{\sim}{\rightarrow} \mathcal{X}_{I}$. Each family $\lambda=\left(\lambda_{i j}\right)_{i \in[q] \backslash I, 1 \leq j \leq n_{i}}$ of non-zero elements of $K$ defines a homomorphism $B_{I}^{\prime} \rightarrow A$ that takes $T_{i 0}$ to $\frac{a_{i}}{\lambda_{i 1} \ldots \cdot \lambda_{i n_{i}}}$ and $T_{i j}$ to $\lambda_{i j}$ for $1 \leq j \leq n_{i}$. The latter together with the isomorphism $\mathcal{X}_{I} \stackrel{\sim}{\rightarrow} \mathcal{V}$ defines a section $\sigma_{\lambda}: \mathcal{X}_{I} \rightarrow$ $f^{-1}\left(\mathcal{X}_{I}\right)$ of $f$ over $\mathcal{X}_{I}$. If $I=[p]$ then, for $\lambda_{i j}=T_{i j}(g(y)), \sigma_{\lambda}(x)=g(y)$. If $I \neq[p]$, we can increase the field $K$ and find a family $\lambda$ such that the point $\sigma_{\lambda}(x)$ is contained in the image of $\mathcal{y}$ in $\mathcal{Z}$. Shrinking $\mathcal{X}$, we can extend the étale morphism $g^{-1}\left(\sigma_{\lambda}\left(\mathcal{X}_{I}\right)\right) \rightarrow \mathcal{X}_{I}$ to an étale morphism $\mathcal{X}^{\prime} \rightarrow \mathcal{X}$ with affine $\mathcal{X}^{\prime}$. Replacing $\mathcal{X}$ by $\mathcal{X}^{\prime}, \mathcal{Y}$ by $\mathcal{X} \times_{X} \mathcal{X}^{\prime}$ and $\mathcal{Z}$ by $\mathcal{Z} \times X_{X} \mathcal{X}^{\prime}$, we may assume that the above section $\sigma_{\lambda}: \mathcal{X}_{I} \rightarrow f^{-1}\left(\mathcal{X}_{I}\right)$ of $f$ over $\mathcal{X}_{I}$ can be lifted to a section $\sigma: \mathcal{X}_{I} \rightarrow \varphi^{-1}\left(\mathcal{X}_{I}\right)$ of $\varphi$ over $\mathcal{X}_{I}$. For $x^{\prime} \in \mathcal{X}_{I}$, let $\mathcal{Y}_{x^{\prime}}^{\circ}$ denote the connected component of $y_{x^{\prime}}$ that contains the 
point $\sigma\left(x^{\prime}\right)$. If $x^{\prime}$ is contained in $\mathcal{X}_{J}$ for some bigger subset $I \subset J \subset[p]$, then the induction hypothesis implies that $y_{x^{\prime}}^{\circ}=y_{x^{\prime}}$. Otherwise, by the construction, the point $\sigma\left(x^{\prime}\right)$ is contained in the intersection of all of the irreducible components of $y_{x^{\prime}}^{\circ}$. If $I=\emptyset$, i.e., $\mathcal{X}_{\emptyset}=\mathcal{X}$, then the union $y^{\circ}$ of $y_{x^{\prime}}$ over all $x^{\prime} \in X$ is open in $y$, by [EGAIV, 15.6.4], and the morphism $y^{\circ} \rightarrow \mathcal{X}$ is geometrically elementary. Assume that $I \neq \emptyset$, and let $I=\left\{i_{0}<\cdots<i_{l}\right\}$ and $\mathbf{n}=\left(n_{i_{0}}, \ldots, n_{i_{l}}\right)$. For a subset $A \subset[\mathbf{n}]$ with the property $A=A_{0} \times \cdots \times A_{l}$, where $A_{k}$ is the image of $A$ under the projection $[\mathbf{n}] \rightarrow\left[n_{i_{k}}\right]$, we set $\mathcal{Z}_{A}=\left\{z \in f^{-1}\left(\mathcal{X}_{I}\right) \mid T_{i j}(z)=0\right.$ for all $i \in I$ and $\left.j \in A_{i}\right\}$ and denote by $\mathcal{Z}_{A}$ the preimage of $\mathcal{Z}_{A}$ in $\mathcal{Z}_{\text {. Then }} \sigma$ is also a section of each of the morphisms $y_{A} \rightarrow \mathcal{X}_{I}$. For a point $x^{\prime} \in \mathcal{X}_{I}$, let $y_{A, x^{\prime}}^{\circ}$ denote the connected component of $y_{A, x^{\prime}}$ that contains the point $\sigma\left(x^{\prime}\right)$, and let $y_{A}^{\circ}$ denote the union of $\mathcal{Y}_{A, x^{\prime}}^{\circ}$ over $x^{\prime} \in \mathcal{X}_{I}$. Again, by [EGAIV, 15.6.4], $y_{A}^{\circ}$ is open in $y_{A}$. Thus, we can remove from $y$ the closed subsets $y_{A} \backslash y_{A}^{\circ}$ so that the fibres of $\varphi$ over all points from $\mathcal{X}_{I}$ are geometrically elementary.

We say that a strictly poly-stable morphism $\varphi: \mathfrak{Y} \rightarrow \mathfrak{X}$ in $k^{\circ}-\mathcal{F} s c h$ is geometrically elementary if the induced morphism between their closed fibres is geometrically elementary. This property implies that for each point $x \in \mathfrak{X}_{\eta}$ the closed fibre of the strictly poly-stable formal scheme $\mathfrak{Y}_{x}$ is elementary. The following is a direct consequence of Lemma 7.3 and [Ber3, Lemma 2.1].

Corollary 7.4. Let $\varphi: \mathfrak{Y} \rightarrow \mathfrak{X}$ be a strictly poly-stable morphism in $k^{\circ}-\mathcal{F}$ sch. Then for each point $\mathbf{y} \in \mathfrak{Y}_{\text {s }}$ there exists an étale morphism $\mathfrak{X}^{\prime} \rightarrow \mathfrak{X}$ and an open subscheme $\mathfrak{Y}^{\prime} \subset \mathfrak{Y} \times \mathfrak{X} \mathfrak{X}^{\prime}$ such that the image of $\mathfrak{Y}_{s}^{\prime}$ in $\mathfrak{Y}_{s}$ contains the point $\mathbf{y}$ and the induced morphism $\mathfrak{Y}^{\prime} \rightarrow \mathfrak{X}^{\prime}$ is geometrically elementary.

Proof of Theorem 7.1. Assume first that the morphism $\varphi$ is strictly polystable. Since the statement on continuity of $\Phi_{\mathfrak{X}}$ is local with respect to the Zariski topology of $\mathfrak{Y}$, we may assume that $\mathfrak{X}=\operatorname{Spf}(A)$ is affine and the morphism $\varphi$ goes through an étale morphism $f: \mathfrak{Y} \rightarrow \mathfrak{Z}=\operatorname{Spf}(B)$, where $B=B_{0} \otimes_{A} \cdots \otimes_{A} B_{p}$ with $B_{i}=A\left\{T_{i 0}, \ldots, T_{i n_{i}}\right\} /\left(T_{i 0} \cdot \ldots \cdot T_{i n_{i}}-a_{i}\right)$, $a_{i} \in A$ and $n_{i} \geq 1$. The group $g=g_{\mathrm{m}}^{\left(n_{0}\right)} \times \cdots \times g_{\mathrm{m}}^{\left(n_{p}\right)}$ from $\S 5$ acts in the evident way on $\mathfrak{Z}$. By Lemma 5.5, the action on $\mathfrak{Z}$ of the formal group $\mathfrak{G}$, the formal completion of $g$ along the unit, extends in a unique way to an action on $\mathfrak{Y}$. The corresponding continuous homotopy $\mathfrak{Y}_{\eta} \times\left[0,1\left[\rightarrow \mathfrak{Y}_{\eta}\right.\right.$ coincides with the restriction of $\Phi_{\mathfrak{X}}$ to $\mathfrak{Y}_{\eta} \times[0,1[$ (see Step 4 from $\S 5$ ). Thus, it remains to check the continuity $\Phi_{\mathfrak{X}}$ at the points $(y, 1) \in \mathfrak{Y}_{\eta} \times[0,1]$. If, in addition, the morphism $\varphi$ is geometrically elementary then, for every point $x \in \mathfrak{X}_{\eta}, \mathfrak{Y}_{x, s}=\mathfrak{Y}_{s, \pi(x)} \otimes_{\widetilde{k}(\pi(x))} \widetilde{\mathscr{H}(x)}$ is elementary and, therefore, the morphism of polysimplicial sets $C\left(\mathfrak{Y}_{x, s}\right) \rightarrow C\left(\mathfrak{Z}_{x, s}\right)$ is injective. Since $S(\mathfrak{Y} / \mathfrak{X})=f_{\eta}^{-1}(S(\mathfrak{Z} / \mathfrak{X}))$, Theorem 5.4 implies that $f_{\eta}^{-1}\left(f_{\eta}(y)\right)=\{y\}$ for all $y \in S(\mathfrak{Y} / \mathfrak{X})$ and, by Lemma 5.10, the map $\Phi_{\mathfrak{X}}$ is continuous. 
In the general case, we can find, by Corollary 7.4, a commutative diagram

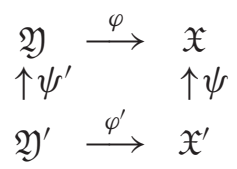

where $\psi^{\prime}$ is surjective étale, $\psi$ is étale, and $\mathfrak{X}^{\prime}$ is a disjoint union $\bigsqcup_{i \in I} \mathfrak{X}_{i}$ such that each morphism $\varphi^{\prime-1}\left(\mathfrak{X}_{i}\right) \rightarrow \mathfrak{X}_{i}$ is strictly poly-stable and geometrically elementary. By the previous case, the map $\Phi_{\mathfrak{X}^{\prime}}: S\left(\mathfrak{Y}^{\prime} / \mathfrak{X}^{\prime}\right) \times$ $[0,1] \rightarrow S\left(\mathfrak{Y}^{\prime} / \mathfrak{X}^{\prime}\right)$ is continuous. Since it is compatible with the map $\Phi_{\mathfrak{X}}$, Lemma 5.11 implies that $\Phi_{\mathfrak{X}}$ is also continuous.

Proof of Theorem 7.2. Step 1. We start by constructing for each $n \geq 0$ a strong deformation retraction $\psi_{n}:[0,1]^{[n]} \times[0,1] \rightarrow[0,1]^{[n]}$ of $[0,1]^{[n]}$ to the point $(1, \ldots, 1)$. The map $\psi_{n}$ is required to possess the property that $\psi(\sigma(\mathbf{r}), t)=\sigma\left(\psi_{n}(\mathbf{r}, t)\right)$ for all $\sigma \in S_{n+1}$, and so it suffices to define $\psi_{n}(\mathbf{r}, t)$ only for the points $\mathbf{r} \in[0,1]^{[n]}$ with $r_{0} \leq r_{1} \leq \cdots \leq r_{n}$. First of all, if $t \leq r_{0} \ldots \cdot r_{n}$ we set $\psi_{n}(\mathbf{r}, t)=\mathbf{r}$. Furthermore, if $r_{i}^{i+1} r_{i+1} \cdot \ldots \cdot r_{n} \leq$ $t<r_{i+1}^{i+2} r_{i+2} \cdot \ldots \cdot r_{n}$ for some $0 \leq i \leq n-1$, we set

$$
\psi_{n}(\mathbf{r}, t)=\left(\left(\frac{t}{r_{i+1} \cdot \ldots \cdot r_{n}}\right)^{\frac{1}{i+1}}, \ldots,\left(\frac{t}{r_{i+1} \cdot \ldots \cdot r_{n}}\right)^{\frac{1}{i+1}}, r_{i+1}, \ldots, r_{n}\right) .
$$

Finally, if $t \geq r_{n}^{n+1}$, we set $\psi_{n}(\mathbf{r}, t)=\left(t^{\frac{1}{n+1}}, \ldots, t^{\frac{1}{n+1}}\right)$. For example, $\psi_{0}(r, t)=\max (r, t)$. Notice that if $\mu_{n}$ denotes the mapping $[0,1]^{[n]} \rightarrow$ $[0,1]:\left(r_{0}, \ldots, r_{n}\right) \mapsto r_{0} \cdot \ldots \cdot r_{n}$, then $\mu_{n}\left(\psi_{n}(\mathbf{r}, t)\right)=\psi_{0}\left(\mu_{n}(\mathbf{r}), t\right)$. One also has $\psi_{n}\left(\psi_{n}(\mathbf{r}, t), t^{\prime}\right)=\psi_{n}\left(\mathbf{r}, \max \left(t, t^{\prime}\right)\right)$.

Step 2. Let $f: \mathfrak{Y}=\operatorname{Spf}(B) \rightarrow \mathfrak{X}=\operatorname{Spf}(A)$ be a morphism of affine formal schemes finitely presented over $k^{\circ}$ with $B=B_{0} \widehat{\otimes}_{A} \ldots \widehat{\otimes}_{A} B_{p}$, where $B_{i}=A\left\{T_{i 0}, \ldots, T_{i n_{i}}\right\} /\left(T_{i 0} \cdot \ldots \cdot T_{i n_{i}}-a_{i}\right), a_{i} \in A$ and $n_{i} \geq 1$. The continuous mapping $\beta_{f}: \mathfrak{Y}_{\eta} \rightarrow \mathfrak{X}_{\eta} \times[0,1]^{[\mathbf{n}]}$ that takes a point $y$ to $\left(f(y) ;\left|T_{00}(y)\right|, \ldots,\left|T_{p n_{p}}(y)\right|\right)$ identifies $S(\mathfrak{Y} / \mathfrak{X})$ with the closed subset (see Step 1 from $§ 5$ )

$$
S_{f}=\left\{\left(x ; \mathbf{r}_{0}, \ldots, \mathbf{r}_{p}\right) \in \mathfrak{X}_{\eta} \times[0,1]^{[\mathbf{n}]}\left|r_{i 0} \ldots . r_{i n_{i}}=\right| a_{i}(x) \mid, 0 \leq i \leq p\right\} .
$$

Assume that there is a homotopy $\Psi: \mathfrak{X}_{\eta} \times[0,1] \rightarrow \mathfrak{X}_{\eta}:(x, t) \mapsto x_{t}$ possessing the properties $\left(x_{t}\right)_{t^{\prime}}=x_{\max \left(t, t^{\prime}\right)}$ and $x \leq x_{t}$ for all $t, t^{\prime} \in[0,1]$. We construct as follows a homotopy $\Psi_{f}: S_{f} \times[0,1] \rightarrow S_{f}$ over $\Psi$ :

$$
\Psi_{f}\left(\left(x ; \mathbf{r}_{0}, \ldots, \mathbf{r}_{p}\right), t\right)=\left(x_{t} ; \psi_{n_{0}}\left(\mathbf{r}_{0},\left|a_{0}\left(x_{t}\right)\right|\right), \ldots, \psi_{n_{p}}\left(\mathbf{r}_{p},\left|a_{p}\left(x_{t}\right)\right|\right)\right) .
$$

In this way we get a homotopy $\widetilde{\Psi}: S(\mathfrak{Y} / \mathfrak{X}) \times[0,1] \rightarrow S(\mathfrak{Y} / \mathfrak{X}): y \mapsto y_{t}$ over $\Psi$. By the construction, $\left(y_{t}\right)_{t^{\prime}}=y_{\max \left(t, t^{\prime}\right)}$ and, if $x=x_{t}$ for $x=f_{\eta}(y)$ then $y=y_{t}$. Since $x \leq x_{t}$ and $\left|T_{i j}(y)\right| \leq\left|T_{i j}\left(y_{t}\right)\right|$ for all $0 \leq i \leq p$ and $0 \leq j \leq n_{i}$, from Lemma 5.6 it follows that $y \leq y_{t}$. Assume that 
$\pi(x)=\pi\left(x_{t}\right)$. This means that, for any element $a \in A,|a(x)|<1$ if and only if $\left|a\left(x_{t}\right)\right|<1$, and we have to check that the same property holds for the points $y$ and $y_{t}$ and all functions from $B$. If such a function is represented by an element $b=\sum_{\mu} a_{\mu} T^{\mu} \in D$ with $a_{\mu} \in A$ (see Lemma 5.6), then the inequality $|b(y)|<1$ (resp. $\left|b\left(y_{t}\right)\right|<1$ ) is equivalent to the system of inequalities $\left|a_{\mu}(x)\right| \cdot|T(y)|^{\mu}<1$ (resp. $\left|a_{\mu}\left(x_{t}\right)\right| \cdot\left|T\left(y_{t}\right)\right|^{\mu}<1$ ) for all $\mu$. If $\left|a_{\mu}(x)\right|<1$ then $\left|a_{\mu}\left(x_{t}\right)\right|<1$ and the both inequalities from the above systems are automatically satisfied. Otherwise, $\left|a_{\mu}(x)\right|=\left|a_{\mu}\left(x_{t}\right)\right|=1$ and the formula for $\Psi_{f}$ implies that $|T(y)|^{\mu}<1$ if and only if $\left|T\left(y_{t}\right)\right|^{\mu}<1$.

We remark that, given an open formal subscheme $\mathfrak{Y}^{\prime} \subset \mathfrak{Y}$, the above homotopy $\widetilde{\Psi}$ induces a homotopy $\widetilde{\Psi}: S\left(\mathfrak{Y}^{\prime} / \mathfrak{X}\right) \times[0,1] \rightarrow S\left(\mathfrak{Y}^{\prime} / \mathfrak{X}\right)$. Indeed, this follows from the facts that $S\left(\mathfrak{Y}^{\prime} / \mathfrak{X}\right)=S(\mathfrak{Y} / \mathfrak{X}) \cap \pi^{-1}\left(\mathfrak{Y}_{s}^{\prime}\right)$ and $y \leq y_{t}$.

Step 3. Let $\varphi: \mathfrak{Z} \rightarrow \mathfrak{X}=\operatorname{Spf}(A)$ be a geometrically elementary strictly poly-stable morphism. Assume that the morphism $\varphi$ is a composition of an étale morphism $g: \mathfrak{Z} \rightarrow \mathfrak{Y}=\operatorname{Spf}(B)$ with a morphism $f: \mathfrak{Y} \rightarrow \mathfrak{X}$ of the form considered in Step 2. Let $\mathfrak{Y}^{\prime}$ be the image of $\varphi$ in $\mathfrak{Y}$. (It is an open formal subscheme of $\mathfrak{Y}$.) We claim that $\varphi$ induces a homeomorphism $S(\mathfrak{Z} / \mathfrak{X}) \stackrel{\sim}{\rightarrow} S\left(\mathfrak{Y}^{\prime} / \mathfrak{X}\right)$. Indeed, it suffices to verify that, for each point $x \in \mathfrak{X}_{\eta}$, the canonical map $S\left(\mathfrak{Z}_{x}\right) \rightarrow S\left(\mathfrak{Y}_{x}^{\prime}\right)$ is a homeomorphism. But $\mathfrak{Z}_{x, s} \stackrel{\sim}{\rightarrow} \mathfrak{Z}_{s, \pi(x)} \otimes_{\tilde{k}(\pi(x))} \widetilde{\mathscr{H}(x)}$ and $\mathfrak{Y}_{x, s}^{\prime} \stackrel{\sim}{\rightarrow} \mathfrak{Y}_{s, \pi(x)}^{\prime} \otimes_{\widetilde{k}(\pi(x))} \widetilde{\mathscr{H}(x)}$. From the assumption it follows that $\mathfrak{Z}_{x, s}$ is elementary. Since $\mathfrak{Y}_{x, s}^{\prime}$ is also elementary, it follows that $C\left(\mathfrak{Z}_{x, s}\right) \stackrel{\sim}{\rightarrow} C\left(\mathfrak{Y}_{x, s}^{\prime}\right)$. Theorem 5.4 implies that $S\left(\mathfrak{Z}_{x}\right) \stackrel{\sim}{\rightarrow} S\left(\mathfrak{Y}_{x}^{\prime}\right)$. Thus, the homotopy from Step 2 gives rise to a homotopy $\widetilde{\Psi}: S(\mathfrak{Z} / \mathfrak{X}) \times[0,1] \rightarrow S(\mathfrak{Z} / \mathfrak{X}): z \mapsto z_{t}$ over $\Psi$. One evidently has $\left(z_{t}\right)_{t^{\prime}}=z_{\max \left(t, t^{\prime}\right)}$ for all $t, t^{\prime} \in[0,1]$ and, if $x=x_{t}$ for some $t$ then $z=z_{t}$. Since $\pi(z) \in \operatorname{str}\left(\mathfrak{Z}_{s, \pi(x)}\right)$ and $\pi\left(z_{t}\right) \in \operatorname{str}\left(\mathfrak{Z}_{s, \pi\left(x_{t}\right)}\right)$, the points $\pi(z)$ and $\pi\left(z_{t}\right)$ are unique preimages of the points $\pi(y)$ and $\pi\left(y_{t}\right)$ in $\mathfrak{Z}_{s}$, respectively, where $y=g_{\eta}(z)$. Since $\pi(y)$ is contained in the closure of $\pi\left(y_{t}\right)$ and $g_{s}: \mathfrak{Z}_{s} \rightarrow \mathfrak{Y}_{s}$ is an open map, it follows that $\pi(z)$ is contained in the closure of $\pi\left(z_{t}\right)$ and if, in addition, $\pi(x)=\pi\left(x_{t}\right)$ for some $t$ then $\pi(z)=\pi\left(z_{t}\right)$.

Step 4. In the situation of Step 3, $z \leq z_{t}$ for all $z \in \mathfrak{Z}_{\eta}$ and $t \in[0,1]$. Since $\pi(z)$ is contained in the closure of $\pi\left(z_{t}\right)$, we can replace $\mathfrak{Z}$ by an open neighborhood of the point $\pi(z)$ so that $\mathfrak{Z}=\operatorname{Spf}\left(C_{\{c\}}\right)$ with $C=B[T] /(P)$, where $P$ is a monic polynomial over $B$ and $c \in C$ is such that the image of $P^{\prime}$ in $C_{\{c\}}$ is invertible. Notice that for $y=g_{\eta}(z)$ one has $y \leq y_{t}, f_{\eta}^{-1}(y)=\{z\}$ and $f_{\eta}^{-1}\left(y_{t}\right)=\left\{z_{t}\right\}$. Notice also that, by Lemma 5.1, the inequality $z \leq z_{t}$ in $\mathfrak{Z}_{\eta}$ is equivalent to the same inequality in the generic fibre of $\operatorname{Spf}(C)$. Thus, the required fact follows from the following lemma.

Lemma 7.5. Let $\varphi: Y=\mathcal{M}(\mathscr{B}) \rightarrow X=\mathcal{M}(\mathcal{A})$ be a finite morphism of $k$-affinoid spaces such that $\mathcal{B}$ is a free $\mathcal{A}$-module, and let $x, x^{\prime} \in X$ be points with $x \leq x^{\prime}$. Furthermore, let $Z$ be an affinoid subdomain of $Y$ of the form $Y\left(h^{-1}\right)=\{y \in Y|| h(y) \mid \geq 1\}$ with $h \in \mathscr{B}^{\circ}$. Assume that $\varphi^{-1}\left(x^{\prime}\right) \cap Z=\left\{z^{\prime}\right\}$. Then for each point $z \in \varphi^{-1}(x) \cap Z$ one has $z \leq z^{\prime}$. 
Proof. (1) For a closed subset $\Sigma \subset Y$ and an element $f \in \mathscr{B}$ we set $|f|_{\Sigma}=\max _{y \in \Sigma}|f(y)|$. We claim that for each $f \in \mathscr{B}$ and each point $x \in X$ one has $|f|_{\varphi^{-1}(x)}=\max _{1 \leq i \leq n}\left|a_{i}(x)\right|^{\frac{1}{i}}$, where $T^{n}+a_{1} T^{n-1}+\cdots+a_{n}$ is the characteristic polynomial of the endomorphism of multiplication by $f$ of $\mathscr{B}$ over $\mathcal{A}$. Indeed, we can replace $\mathcal{A}$ by $\mathscr{H}(x)$ and $\mathscr{B}$ by $\mathscr{B} \otimes_{\mathcal{A}} \mathcal{H}(x)$ so that the claim takes the following form. Given a non-Archimedean field $K$ and a finite-dimensional algebra $L$ over $K$, for each $f \in L$ one has $|f|_{\text {sup }}=$ $\max _{1 \leq i \leq n}\left|a_{i}(x)\right|^{\frac{1}{i}}$, where $T^{n}+a_{1} T^{n-1}+\cdots+a_{n}$ is the characteristic polynomial of $f$. Notice that the both sides of the equality don't change if we increase the $K$ and, therefore, we may assume that $L=L_{1} \times \cdots \times L_{p}$, where $L_{i}$ are local Artinian $K$-algebras with the residue field $K$.

Let $\lambda_{i}$ be the image of $f$ in the residue field of $L_{i}$. The left hand side of the equality is $\max _{1 \leq i \leq p}\left|\lambda_{i}\right|$. On the other hand, if the dimension of $L_{i}$ over $K$ is $n_{i}$, the characteristic polynomial of $f$ is $\left(T-\lambda_{1}\right)^{n_{1}} \ldots \cdot\left(T-\lambda_{p}\right)^{n_{p}}$, and the verification of the equality is an easy exercise.

(2) Assume we are given a point $x \in X$ with $\varphi^{-1}(x) \cap Z=\{z\}$ and a function $f \in \mathscr{B}$. We claim that if $f(z) \neq 0$ then there exists $m_{0} \geq 1$ such that for any $m \geq m_{0}$ one has $|f(z)|=\left|f h^{m}\right|_{\varphi^{-1}(x)}$, and if $f(z)=0$ then $\left|f h^{m}\right|_{\varphi^{-1}(x)} \rightarrow 0$ as $m \rightarrow \infty$. Indeed, if $\varphi^{-1}(x)=\left\{y_{1}=z, y_{2}, \ldots, y_{l}\right\}$, then $|h(z)|=1$ and $\left|h\left(y_{i}\right)\right|<1$ for all $2 \leq i \leq l$ and, therefore, in the case $f(z)=0$ the claim is clear, and in the case $f(z) \neq 0$ it suffices to take $m_{0}$ with the property $\left|f\left(y_{i}\right)\right| \cdot\left|h\left(y_{i}\right)\right|^{m_{0}} \leq|f(z)|$ for all $2 \leq i \leq l$.

(3) For each $f \in \mathscr{B}$ one has $|f(z)| \leq\left|f\left(z^{\prime}\right)\right|$. (The points $z$ and $z^{\prime}$ are from the lemma.) Indeed, by (1) and the assumption $x \leq x^{\prime}$, for every $m \geq 0$ one has $|f(z)|=\left|\left(f h^{m}\right)(z)\right| \leq\left|f h^{m}\right|_{\varphi^{-1}(x)} \leq\left|f h^{m}\right|_{\varphi^{-1}\left(x^{\prime}\right)}$. If $f\left(z^{\prime}\right) \neq 0$ then for a sufficiently large $m$ the latter number is equal to $\left|f\left(z^{\prime}\right)\right|$. If $f\left(z^{\prime}\right)=0$ then that number tends to zero as $m$ tends to infinity, i.e., $f(z)=0$.

Step 5. In the situation of Step 3, the homotopy $\widetilde{\Psi}: S(\mathfrak{Z} / \mathfrak{X}) \times[0,1] \rightarrow$ $S(\mathfrak{Z} / \mathfrak{X})$ does not depend on the decomposition $\varphi=f \circ g$.

Lemma 7.6. Let $[0,1] \rightarrow \mathfrak{X}_{\eta}: x \mapsto x_{t}$ be a continuous map to the generic fibre of a formal scheme $\mathfrak{X}$ locally finitely presented over $k^{\circ}$, and assume that $x_{t} \leq x_{t^{\prime}}$ for all $t \leq t^{\prime}$. Then there exist $t_{0}=0<t_{1}<\cdots<t_{n}=1$ such that $\pi\left(x_{t}\right)=\pi\left(x_{t_{i}}\right)$ for all $t_{i} \leq t<t_{i+1}$ and $0 \leq i \leq n-1$.

Proof. We may assume that $\mathfrak{X}=\operatorname{Spf}(A)$ is affine, and it suffices to show that for each $0 \leq t<1$ there exists $\varepsilon>0$ such that $\pi\left(x_{t}\right)=\pi\left(x_{t^{\prime}}\right)$ for all $t \leq t^{\prime}<t+\varepsilon$. Let $f_{1}, \ldots, f_{m}$ be elements of $A$ whose images in $\widetilde{A}$ generate the prime ideal $\wp$ that corresponds to the point $\pi\left(x_{t}\right)$. Since the map $t \mapsto x_{t}$ is continuous and $\left|f_{i}\left(x_{t}\right)\right|<1$ for all $1 \leq i \leq m$, there exists $\varepsilon>0$ such that $\left|f_{i}\left(x_{t^{\prime}}\right)\right|<1$ for all $t \leq t^{\prime}<t+\varepsilon$ and $1 \leq i \leq m$. We claim that for such $t^{\prime}$ one has $\pi\left(x_{t}\right)=\pi\left(x_{t^{\prime}}\right)$. Indeed, if $f$ is an element of $A$ whose image 
in $\widetilde{A}$ is not in $\wp$, then $\left|f\left(x_{t}\right)\right|=1$ and, therefore, $\left|f\left(x_{t^{\prime}}\right)\right| \geq\left|f\left(x_{t}\right)\right|=1$. This means that $\pi\left(x_{t}\right)=\pi\left(x_{t^{\prime}}\right)$.

Assume we are given another decomposition $\varphi=f^{\prime} \circ g^{\prime}$, where $g^{\prime}$ : $\mathfrak{Z} \rightarrow \mathfrak{Y}^{\prime}=\operatorname{Spf}\left(B^{\prime}\right)$ is an étale morphism with $B^{\prime}=B_{0}^{\prime} \widehat{\otimes}_{A} \ldots \widehat{\otimes}_{A} B_{p^{\prime}}^{\prime}$, where $B_{i}^{\prime}=A\left\{T_{i 0}, \ldots, T_{i n_{i}^{\prime}}\right\} /\left(T_{i 0} \cdot \ldots \cdot T_{i n_{i}^{\prime}}-a_{i}^{\prime}\right), a_{i}^{\prime} \in A$ and $n_{i}^{\prime} \geq 1$. First of all, we remark that the required fact is true if $f^{\prime}=h \circ f$, where $h$ is the isomorphism $\mathfrak{Y} \stackrel{\sim}{\rightarrow} \mathfrak{Y}{ }^{\prime}$ induced by an isomorphism $[\mathbf{n}] \stackrel{\sim}{\rightarrow}\left[\mathbf{n}^{\prime}\right]$. Let $z \in \underset{\sim}{S}(\mathfrak{Z} / \mathfrak{X})$ and $x=\varphi(z)$. Since the maps $t \mapsto z_{t}=\widetilde{\Psi}(z, t)$ and $t \mapsto z_{t}^{\prime}=\widetilde{\Psi}^{\prime}(z, t)$ are continuous, it follows from Lemma 7.6 that it suffices to verify the equality $z_{t}=z_{t}^{\prime}$ under the additional assumptions that $\pi\left(x_{t}\right)=\pi(x)$ and $\pi\left(z_{t}\right)=\pi(z)=\pi\left(z_{t}^{\prime}\right)$. We can shrink $\mathfrak{X}$ and $\mathfrak{Z}$ and replace $f$ and $f^{\prime}$ by their compositions with isomorphisms of the above type so that there exist $q \geq 0$ and $\mathbf{m}=\left(m_{0}, \ldots, m_{q}\right) \in \mathrm{Ob}(\boldsymbol{\Lambda})$ for which $\left|a_{i}(x)\right|<1$ and $\left|a_{i}^{\prime}(x)\right|<1$ precisely for $0 \leq i \leq q$ and $\left|g^{*} T_{i k}(z)\right|<1$ and $\left|g^{\prime *} T_{i k}^{\prime}(z)\right|<1$ precisely for $0 \leq i \leq q$ and $0 \leq k \leq m_{i}$. Let $\mathbf{z}=\pi(z)$ and $\mathbf{x}=\pi(x)$. The étale morphisms $g$ and $g^{\prime}$ give rise to two isometric bijections $\mu, \mu^{\prime}$ : $[\mathbf{m}] \stackrel{\sim}{\rightarrow} \operatorname{irr}\left(\mathfrak{Z}_{s, \mathbf{x}}, \mathbf{z}\right)$. Replacing again, if necessary, $f^{\prime}$ by its composition with the automorphism of $\mathfrak{Y}^{\prime}$ induced by an automorphism of [m], we may assume that $\mu^{\prime}=\mu$. Proposition 4.3 implies that $\left|a_{i}\left(x^{\prime}\right)\right|=\left|a_{i}^{\prime}\left(x^{\prime}\right)\right|$ for all $x^{\prime} \in \pi^{-1}(\mathbf{x})$ and $0 \leq i \leq q$. It follows that for each point $x^{\prime} \in \pi^{-1}(\mathbf{x})$ the two homeomorphisms $S\left(\mathfrak{Z}_{x^{\prime}, \eta}\right) \stackrel{\sim}{\rightarrow} \Sigma_{\left|\mathbf{a}\left(x^{\prime}\right)\right|}^{\mathbf{m}}$, induced by $g$ and $g^{\prime}$, coincide. The latter immediately implies that $z_{t}=z_{t}^{\prime}$ for all $t$ we consider.

Step 6. Assume we are given a second strictly poly-stable morphism $\varphi^{\prime}: \mathfrak{Z}^{\prime} \rightarrow \mathfrak{X}^{\prime}=\operatorname{Spf}\left(A^{\prime}\right)$, satisfying the same properties from Step 3 as the morphism $\varphi$, and a commutative diagram in $\mathcal{F} s c h$

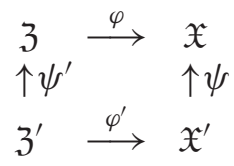

such that the morphism $\mathfrak{Z}^{\prime} \rightarrow \mathfrak{Z} \times \mathfrak{X} \mathfrak{X}^{\prime}$ is étale. Assume also that there is a homotopy $\Psi^{\prime}: \mathfrak{X}_{\eta}^{\prime} \times[0,1] \rightarrow \mathfrak{X}_{\eta}^{\prime}$ possessing the same properties from Step 2 as the homotopy $\Psi$ and such that $\psi_{\eta}\left(\Psi^{\prime}\left(x^{\prime}, t\right)\right)=\Psi^{\prime}\left(\psi_{\eta}\left(x^{\prime}\right), t\right)$ for all $x^{\prime} \in \mathfrak{X}_{\eta}^{\prime}$ and $t \in[0,1]$. Then $\psi_{\eta}^{\prime}\left(\widetilde{\Psi}^{\prime}\left(z^{\prime}, t\right)\right)=\widetilde{\Psi}\left(\psi_{\eta}^{\prime}\left(z^{\prime}\right), t\right)$ for all $z^{\prime} \in \mathfrak{Z}_{\eta}^{\prime}$ and $t \in[0,1]$. Indeed, it suffices to consider the following two cases: (a) $\mathfrak{X}^{\prime}=\mathfrak{X}$, and (b) $\mathfrak{Z}^{\prime}=\mathfrak{Z} \times \mathfrak{X} \mathfrak{X}^{\prime}$. In the case (a) the statement straightforwardly follows from Step 5. In the case (b) the statement follows from the formula for the homotopy $\Psi_{f}$ from Step 2.

Step 7. Let us return to the situation of the theorem. For a poly-stable morphism $\varphi: \mathfrak{Y} \rightarrow \mathfrak{X}$ we construct as follows a strictly poly-stable mor- 
phism $\varphi^{\prime}: \mathfrak{Y}^{\prime} \rightarrow \mathfrak{X}^{\prime}$ a commutative diagram

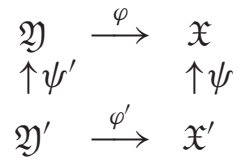

By Corollary 7.4, one can find for each point $\mathbf{y} \in \mathfrak{Y}_{s}$ an étale morphism $\mathfrak{U}=\operatorname{Spf}(A) \rightarrow \mathfrak{X}$ and an étale morphism $\mathfrak{V} \rightarrow \mathfrak{Y} \times \mathfrak{X} \mathfrak{U}$ such that $\mathfrak{V} \rightarrow \mathfrak{U}$ is a geometrically elementary strictly poly-stable morphism and the image of $\mathfrak{V}_{s}$ in $\mathfrak{Y}_{s}$ contains the point $\mathbf{y}$. The commutative diagram we are talking about is obtained for $\mathfrak{Y}^{\prime}$, a disjoint union of $\mathfrak{V}$ 's of the above form so that the induced étale morphism $\mathfrak{Y}^{\prime} \rightarrow \mathfrak{Y}$ is surjective, and for $\mathfrak{X}^{\prime}$, a disjoint union of the corresponding $\mathfrak{U}$ 's.

Step 8. For a poly-stable morphism $\varphi: \mathfrak{Y} \rightarrow \mathfrak{X}$ we construct the required homotopy $\widetilde{\Psi}: S(\mathfrak{Y} / \mathfrak{X}) \times[0,1] \rightarrow S(\mathfrak{Y} / \mathfrak{X})$ as follows. Take a strictly polystable morphism $\mathfrak{Y}^{\prime} \rightarrow \mathfrak{X}^{\prime}$ as constructed in Step 7. Furthermore, take a strictly poly-stable morphism $\mathfrak{Y}^{\prime \prime} \rightarrow \mathfrak{X}^{\prime \prime}$ as constructed in Step 7 for the morphism $\mathfrak{Y}^{\prime} \times \mathfrak{Y} \times \mathfrak{Y}^{\prime} \rightarrow \mathfrak{X}^{\prime} \times \mathfrak{X} \mathfrak{X}^{\prime}$. Since $S\left(\mathfrak{Y}^{\prime} / \mathfrak{X}^{\prime}\right)$ and $S\left(\mathfrak{Y}^{\prime \prime} / \mathfrak{X}^{\prime \prime}\right)$ are the preimages of $S(\mathfrak{Y} / \mathfrak{X})$ in $\mathfrak{Y}_{\eta}^{\prime}$ and $\mathfrak{Y}_{\eta}^{\prime \prime}$, respectively, Lemma 5.11 implies

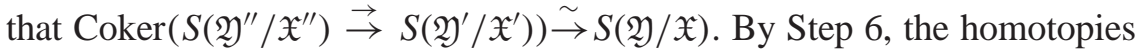
constructed in Step 3 for $S\left(\mathfrak{Y}^{\prime \prime} / \mathfrak{X}^{\prime \prime}\right)$ and $S\left(\mathfrak{Y}^{\prime} / \mathfrak{X}^{\prime}\right)$ are compatible and, therefore, they induce a homotopy $\widetilde{\Psi}: S(\mathfrak{Y} / \mathfrak{X}) \times[0,1] \rightarrow S(\mathfrak{Y} / \mathfrak{X})$. That the homotopy $\widetilde{\Psi}$ satisfies the properties (i)-(iii) follows from the construction.

\section{The homotopy type of the generic fibre of a poly-stable fibration of formal schemes}

The results of the previous section can be used to construct, for each formal scheme $\mathfrak{S}$ in $\mathcal{F}$ sch and each poly-stable fibration $\underline{\mathfrak{X}}=\left(\mathfrak{X}_{l} \stackrel{f_{l-1}}{\rightarrow} \cdots \stackrel{f_{1}}{\rightarrow}\right.$ $\left.\mathfrak{X}_{1} \stackrel{f_{0}}{\rightarrow} \mathfrak{X}_{0}=\mathfrak{S}\right)$, a homotopy $\Phi_{\mathfrak{S}}^{l}: \mathfrak{X}_{l, \eta} \times[0, l] \rightarrow \mathfrak{X}_{l, \eta}:(x, t) \mapsto x_{t}$ over $\mathfrak{S}_{\eta}$. Namely, if $l=1$, it is the homotopy $\Phi_{\mathfrak{S}}$ from Theorem 7.1. Assume that $l \geq 2$ and that the homotopy $\Phi_{\mathfrak{S}}^{l-1}$ is already constructed. First of all, we set $x_{t}=\Phi_{\mathfrak{X}_{l-1}}(x, t)$ for $0 \leq t \leq 1$. Furthermore, given a morphism $\left(k^{\prime}, \mathfrak{X}^{\prime}\right) \rightarrow\left(k, \mathfrak{X}_{l-1}\right)$ in $\mathcal{F} s c h^{\text {ét }}$, let $\underline{\mathfrak{X}}^{\prime}$ be the poly-stable fibration $\left(\mathfrak{X}^{\prime} \rightarrow \mathfrak{X}_{l-2} \widehat{\otimes} k^{\prime \circ} \rightarrow \cdots \rightarrow \mathfrak{X}_{0} \widehat{\otimes} k^{\prime \circ}\right)$. By induction, there is a proper strong deformation retraction $\Phi_{\mathfrak{S}}^{l-1}: \mathfrak{X}_{\eta}^{\prime} \times[0, l-1] \rightarrow \mathfrak{X}_{\eta}^{\prime}$. Setting $\Psi_{\mathfrak{X}^{\prime}}=\Phi_{\mathfrak{S}}^{l-1}$, we get a system of homotopies that satisfy the properties (a) and (b) from $\S 7$. Theorem 7.2 provides therefore a homotopy $\widetilde{\Psi}: S\left(\mathfrak{X}_{l} / \mathfrak{X}_{l-1}\right) \times[0, l-1] \rightarrow$ $S\left(\mathfrak{X}_{l} / \mathfrak{X}_{l-1}\right)$ over $\Phi_{\mathfrak{S}}^{l-1}$, and we set $x_{t}=\widetilde{\Psi}\left(x_{1}, t-1\right)$ for all $1 \leq t \leq l$. The homotopy $\Phi_{\mathfrak{S}}^{l}$ is a proper strong deformation retraction of $\mathfrak{X}_{l, \eta}$ to a closed subset which is naturally to be called the skeleton of $\underline{\mathfrak{X}}$ and to be denoted 
by $S(\underline{\mathfrak{X}})$. The following theorem lists properties of the homotopy and the skeleton for $\mathfrak{S}$ of the form $\operatorname{Spf}\left(k^{\circ}\right)$.

Theorem 8.1. One can construct for every poly-stable fibration $\underline{\mathfrak{X}}=\left(\mathfrak{X}_{l} \stackrel{f_{l-1}}{\rightarrow}\right.$ $\left.\ldots \stackrel{f_{1}}{\rightarrow} \mathfrak{X}_{1}\right)$ of length $l$ a proper strong deformation retraction $\Phi^{l}: \mathfrak{X}_{l, \eta} \times$ $[0, l] \rightarrow \mathfrak{X}_{l, \eta}:(x, t) \mapsto x_{t}$ of $\mathfrak{X}_{l, \eta}$ to the skeleton $S(\underline{\mathfrak{X}})$ of $\underline{\mathfrak{X}}$, so that the following holds:

(i) $S(\underline{\mathfrak{X}})=S\left(\mathfrak{X}_{l} / \mathfrak{X}_{l-1}\right) \cap f_{l-1}^{-1}\left(S\left(\underline{\mathfrak{X}}^{\prime}\right)\right)$, where $\underline{\mathfrak{X}}^{\prime}=\left(\mathfrak{X}_{l-1} \stackrel{f_{l-2}}{\rightarrow} \cdots \stackrel{f_{1}}{\rightarrow} \mathfrak{X}_{1}\right)$, and $f_{l-1, \eta}\left(x_{t}\right)=f_{l-1, \eta}(x)_{t-1}$ for all $1 \leq t \leq l$;

(ii) $\left(x_{t}\right)_{t^{\prime}}=x_{\max \left(t, t^{\prime}\right)}$ for all $0 \leq t, t^{\prime} \leq l$;

(iii) $x \leq x_{t}$ for all $0 \leq t \leq l$;

(iv) for every $x$ and $0 \leq i \leq l-1$, there exists $i \leq t^{\prime} \leq i+1$ such that $x_{t}=x_{i}$ for all $i \leq t \leq t^{\prime}$ and the map $\left[t^{\prime}, i+1\right] \rightarrow \mathfrak{X}_{\eta}: t \mapsto x_{t}$ is injective;

(v) for every $0 \leq t \leq l, \pi\left(x_{t}\right)$ is contained in the same stratum of $\mathfrak{X}_{l, s}$ as $\pi(x)$; moreover, $\pi\left(x_{l}\right)$ is the generic point of that stratum, and $\pi\left(x_{i}\right)=\pi\left(x_{t}\right)$ for all $0 \leq i \leq l-1$ and $i \leq t<i+1$;

(vi) if $\mathfrak{X}_{l, \eta}$ is normal at $x$, then for every open affine subscheme $\mathfrak{X}^{\prime} \subset \mathfrak{X}_{l}$ with $x \in \mathfrak{X}_{\eta}^{\prime}$ and every $l-1<t \leq l$ the local ring $\mathcal{O}_{\mathfrak{X}_{\eta}^{\prime}, x_{t}}$ is a field;

(vii) if $\underline{\mathfrak{X}}$ is a strictly poly-stable fibration then, for every point $x \in S(\underline{\mathfrak{X}})$, $\widetilde{\mathscr{H}(x)}$ is a purely transcendent extension of the field $\widetilde{k}(\pi(x))$;

(viii) given a morphism $\varphi: \underline{Y} \rightarrow \underline{\mathfrak{X}}$ in $\mathcal{P}_{\text {st }} f_{l}^{e t}$, one has $\varphi_{l, \eta}\left(y_{t}\right)=\varphi_{l, \eta}(y)_{t}$ for all $y \in \mathfrak{Y}_{l, \eta}$ and $0 \leq t \leq l$; if $\varphi$ is étale then, given $x \in \mathfrak{X}_{\eta}, 0 \leq i \leq l-1$ and $i \leq t<i+1$, each point from $\varphi_{l, \eta}^{-1}\left(x_{t}\right)$ is of the form $y_{t}$ for some $y \in \varphi_{l, \eta}^{-1}\left(x_{i}\right)$.

Let $S^{l}$ denote the functor $\underline{\mathfrak{x}} \mapsto S(\underline{\mathfrak{X}})$ from $\mathcal{P} s t f_{l}^{\mathrm{tps}}$ to the category of locally compact spaces. The functor $\mathcal{P} s t f_{l}^{\mathrm{tps}} \rightarrow \boldsymbol{\Lambda}^{\circ} \mathcal{E} n s: \underline{\mathfrak{x}} \mapsto \mathbf{C}\left(\underline{\mathfrak{X}}_{s}\right)$ will be denoted by $\mathbf{C}^{l}$, and its composition with the geometric realization functor $\underline{\mathfrak{x}} \mapsto\left|\mathbf{C}\left(\underline{\mathfrak{X}}_{s}\right)\right|$ will be denoted by $\left|\mathbf{C}^{l}\right|$.

Theorem 8.2. There is a canonical isomorphism of functors $\left|\mathbf{C}^{l}\right| \stackrel{\sim}{\rightarrow} S^{l}$ that possesses the following property: given a poly-stable fibration $\underline{\mathfrak{X}}$, the homeomorphism $|\mathbf{C}(\underline{\mathfrak{X}})| \stackrel{\sim}{\rightarrow} S(\underline{\mathfrak{X}})$ induces a homeomorphism $|\mathbf{C}(\mathcal{X})| \stackrel{\sim}{\rightarrow} S(\underline{\mathfrak{X}}) \cap$ $\pi^{-1}(\mathcal{X})$ for each strata subset $\mathcal{X}$ of $\mathfrak{X}_{l, s}$.

Here again $|\mathbf{C}(\mathcal{X})|$ denotes the subset of $|\mathbf{C}(\underline{\mathfrak{x}})|$ which is a union of the cells that correspond to the strata of $\mathfrak{X}_{l, s}$ contained in $\mathcal{X}$.

Proof of Theorem 8.1. The construction of the homotopy $\Phi^{l}$ has been already done. That it satisfies the properties (i)-(iv), the third part of (v) and the first part of (viii) straightforwardly follows from Theorems 7.1 and 7.2. As for the second part of (viii), it is obtained using the fact, that $\pi\left(x_{i}\right)=\pi\left(x_{t}\right)$ for all $0 \leq i \leq l-1$ and $i \leq t<i+1$, and the reasoning from $\S 5$, Step 12, that establishes the corresponding statement (vii) of Theorem 5. 
(v) The first two statements of (v) are proven by induction on $l$. Since their validity is local with respect to the étale topology of $\mathfrak{X}_{l}$, it suffices to consider the case when $\mathfrak{X}_{l-1}=\operatorname{Spf}(A)$ and $\mathfrak{X}_{l}=\operatorname{Spf}(B)$ with $B=B_{0} \widehat{\otimes}_{A} \ldots \widehat{\otimes}_{A} B_{p}$ with $B_{i}=A\left\{T_{i 0}, \ldots, T_{i n_{i}}\right\} /\left(T_{i 0} \cdot \ldots \cdot T_{i n_{i}}-a_{i}\right)$, where $a_{i} \in A$ and $n_{i} \geq 1$. We may also assume that $\mathfrak{X}_{l, s}$ is elementary and that the generic point $\mathbf{x}$ of the stratum $\mathcal{X}$ that contains $\pi(x)$ is the unique maximal point in $\operatorname{str}\left(\mathfrak{X}_{l, s}\right)$. Let $\mathcal{X}^{\prime}$ be the stratum of $\mathfrak{X}_{l-1, s}$ that contains the image of $\mathcal{X}$, and let $\mathbf{x}^{\prime}$ be its generic point. We may assume that $a_{i}\left(\mathbf{x}^{\prime}\right)=0$ for $0 \leq i \leq q$ and $a_{i}\left(\mathbf{x}^{\prime}\right) \neq 0$ for $q+1 \leq i \leq p$. Then $\mathcal{X}$ is the subset of $f_{l-1, s}^{-1}\left(\mathcal{X}^{\prime}\right)$ where the functions $T_{i j}$ for $0 \leq i \leq q$ and $0 \leq j \leq n_{i}$ vanish. It follows that $\pi^{-1}(\mathcal{X})=\left\{x \in f_{l-1, \eta}^{-1}\left(\pi^{-1}\left(\mathcal{X}^{\prime}\right)\right)|| T_{i j}(x) \mid<1\right.$ for all $0 \leq i \leq q$ and $\left.0 \leq j \leq n_{i}\right\}$. From Theorem 5.2 it follows that for $t \in[0,1]$ the point $x_{t}$ is contained in $\pi^{-1}(\mathcal{X})$ and $x_{1}$ is contained in $S\left(\mathfrak{X}_{l} / \mathfrak{X}_{l-1}\right) \cap \pi^{-1}(\mathcal{X})$. We may therefore assume that $x=x_{1}$. The formula of Step 2 from the proof of Theorem 7.2 and the induction hypothesis imply that for $t \in[1, l]$ the point $x_{t}$ is contained in $\pi^{-1}(\mathcal{X})$, and $x_{l}$ is contained in the preimage of $S\left(\mathfrak{X}^{\prime}\right) \cap \pi^{-1}\left(\mathbf{x}^{\prime}\right)$ in $\pi^{-1}(\mathcal{X})$, where $\mathfrak{X}^{\prime}=\left(\mathfrak{X}_{l-1} \rightarrow \cdots \rightarrow \mathfrak{X}_{1}\right)$. The latter means that for $x^{\prime}=f_{l-1, \eta}\left(x_{l}\right)$ one has $\pi\left(x^{\prime}\right)=\mathbf{x}^{\prime}$. Since $x_{l}=\Phi_{\mathfrak{X}_{l-1}}\left(x_{l}, 1\right)$, Proposition 2.7 and Theorem 5.2, applied to the poly-stable formal scheme $\mathfrak{X}_{l} \times \mathfrak{X}_{l-1} \operatorname{Spf}\left(\mathscr{H}\left(x^{\prime}\right)^{\circ}\right)$, imply that $\pi\left(x_{l}\right)=\mathbf{x}$.

(vi) We prove by induction on $l$ the following slightly more strong statement. For every normal point $x \in \mathfrak{X}_{l, x}$ and every $l-1<t \leq l$, there exists a morphism $\mathfrak{Y} \rightarrow \mathfrak{X}_{l}$ in $\mathcal{F}$ sch ${ }^{e}$ with affine $\mathfrak{Y}$ such that $x_{t}$ is the image of a point $y \in \mathfrak{Y}_{\eta}$ which is a unique maximal point of an affinoid domain $Y \subset \mathfrak{Y}_{\eta}$. If $l=1$, it is exactly the fact established in Step 11 of the proof of Theorem 5.2. Assume that $l \geq 2$. For $x^{\prime}=f_{l-1, \eta}(x)$ one has $f_{l-1, \eta}\left(x_{t}\right)=x_{t-1}^{\prime}$. By induction, we may assume that $\mathfrak{X}_{l-1}=\operatorname{Spf}(A)$ is affine and $x_{t-1}^{\prime}$ is a unique maximal point of an affinoid domain $X \subset \mathfrak{X}_{l-1, \eta}$. We may also assume that the morphism $f_{l-1}$ is geometrically elementary and factors through an étale morphism $\mathfrak{X}_{l} \rightarrow \mathfrak{Z}=\operatorname{Spf}(B)$ with $B=$ $B_{0} \widehat{\otimes}_{A} \ldots \widehat{\otimes}_{A} B_{p}$, where $B_{i}=A\left\{T_{i 0}, \ldots, T_{i n_{i}}\right\} /\left(T_{i 0} \cdot \ldots \cdot T_{i n_{i}}-a_{i}\right), a_{i} \in A$ and $n_{i} \geq 1$. Since the point $x^{\prime}$ is normal, the point $x_{t-1}^{\prime}$ is also normal and, therefore, the functions $a_{i}$ do not vanish at $x_{t-1}^{\prime}$. We shrink the affinoid domain $X$ so that the absolute value of each of the functions $a_{i}$ is a non-zero constant on $X$. Furthermore, let $z$ be the image of the point $x_{t}$ in $\mathfrak{Z}_{\eta}$. One has $z \in S\left(\mathfrak{Z} / \mathfrak{X}_{l-1}\right)$. We set $Z=\left\{z^{\prime} \in g_{\eta}^{-1}(X)|| T_{i j}\left(z^{\prime}\right)|=| T_{i j}(z) \mid\right.$ for all $0 \leq i \leq p$ and $0 \leq j \leq n_{i}$, where $g$ denotes the morphism $\mathfrak{Z} \rightarrow \mathfrak{X}_{l-1}$. The affinoid domain $Z$ is isomorphic to a direct product of $X$ with onedimensional annuli, and $z$ is evidently its unique maximal point. Notice that $x_{t}$ is a unique preimage of the point $z$ in $\mathfrak{X}_{l, \eta}$ since the morphism $f_{l-1}$ is geometrically elementary. Furthermore, we can shrink $\mathfrak{X}_{l}=\operatorname{Spf}(C)$ and assume that $C=(B[T] /(P))_{\{b\}}$, where $b \in B[T] /(P)$ and $P$ is a monic polynomial for which the image of $P^{\prime}$ in $C$ is invertible. Since $z$ is a unique maximal point of $Z$, from Lemma 7.5 it follows that $x_{t}$ is a unique maximal point of $Y$, the preimage of $Z$ in $\mathfrak{X}_{l, \eta}$. 
(vii) follows from the following lemma.

Lemma 8.3. Let $\varphi: \mathfrak{Y} \rightarrow \mathfrak{X}$ be a strictly poly-stable morphism between formal schemes locally finitely presented over $k^{\circ}$, and let $y \in S(\mathfrak{Y} / \mathfrak{X})$ and $x=\varphi_{\eta}(y)$. If that $\widetilde{\mathcal{H}(x)}$ is a purely transcendent extension of $\widetilde{k}(\pi(x))$, then $\widetilde{\mathscr{H}(y)}$ is a purely transcendent extension of $\widetilde{k}(\pi(y))$.

Proof. Let $\mathbf{x}=\pi(x)$ and $\mathbf{y}=\pi(y)$. Furthermore, let $\mathfrak{Y}_{x}=\mathfrak{Y} \times \mathfrak{X} \operatorname{Spf}\left(\mathscr{H}(x)^{\circ}\right)$. One has $\mathfrak{Y}_{x, \eta} \stackrel{\sim}{\rightarrow} \mathfrak{Y}_{\eta, x}$ and $\mathfrak{Y}_{x, s} \stackrel{\sim}{\rightarrow} \mathfrak{Y}_{s, \mathbf{x}} \otimes_{\widetilde{k}(\mathbf{x})} \widetilde{\mathscr{H}(x)}$. Since $\widetilde{\mathcal{H}(x)}$ is purely transcendent over $\widetilde{k}(\mathbf{x})$, it follows that $\operatorname{str}\left(\mathfrak{Y}_{x, s}\right) \stackrel{\sim}{\rightarrow} \operatorname{str}\left(\mathfrak{Y}_{s, \mathbf{x}}\right)$ and, if $\mathbf{y}^{\prime}$ denotes the preimage of the point $\mathbf{y}$ under the latter bijection, the field $\widetilde{\mathcal{H}(x)}\left(\mathbf{y}^{\prime}\right)$ is purely transcendent over $\widetilde{k}(\mathbf{y})$. By Theorem 5.2(vi) applied to $\mathfrak{Y}_{x}, \widetilde{\mathscr{H}(y)}$ is purely transcendent over $\widehat{\mathscr{H}(x)}\left(\mathbf{y}^{\prime}\right)$. Thus, $\widetilde{\mathscr{H}(y)}$ is purely transcendent over $\widetilde{k}(\mathbf{y})$.

Proof of Theorem 8.2. Step 1. Fix a number $0<\rho<1$. For a point $\mathbf{u}=\left(u_{0}, \ldots, u_{n}\right) \in \Sigma^{n} \subset[0,1]^{[n]}$ and $r \in[0,1]$, let $\phi_{n}(\mathbf{u}, r)$ denote the unique point $\mathbf{v}=\left(v_{0}, \ldots, v_{n}\right) \in[0,1]^{[n]}$ which lies on the line connecting the points $\rho^{\mathbf{u}}=\left(\rho^{u_{0}}, \ldots, \rho^{u_{n}}\right)$ and $(1, \ldots, 1)$ and satisfies the equality $v_{0} \cdot \ldots \cdot v_{n}=r$. In this way we get a continuous mapping $\phi_{n}: \Sigma^{n} \times[0,1] \rightarrow$ $[0,1]^{[n]}$.

Step 2. Suppose we are given an affine strictly pluri-nodal formal scheme $\mathfrak{X}=\operatorname{Spf}(A)$ over $k^{\circ}$ and a polysimplicial set $E$ provided with a map of partially ordered sets $O(E) \rightarrow \operatorname{str}\left(\mathfrak{X}_{s}\right):(\boldsymbol{\Lambda}[\mathbf{c}] \stackrel{\alpha}{\rightarrow} E) \mapsto \bar{\alpha}$ and a continuous map $\delta:|E| \rightarrow \mathfrak{X}_{\eta}$ possessing the following two properties: (a) $(\boldsymbol{\Lambda}[\mathbf{c}] \stackrel{\alpha}{\rightarrow} E) \in O(E)$ the image of $\stackrel{\circ}{\Sigma}^{\mathbf{c}}$ under the corresponding map to $\mathfrak{X}_{\eta}$ is contained in $\pi^{-1}(\bar{\alpha})$ and (b) for each point $x \in \operatorname{Im}(\delta)$, the field $\widetilde{\mathscr{H}(x)}$ is purely transcendent over $\widetilde{k}(\pi(x))$. Furthermore, let $\varphi$ : $\mathfrak{Y}=\operatorname{Spf}(B) \rightarrow \mathfrak{X}$ be a morphism with $B=B_{0} \widehat{\otimes}_{A} \ldots \widehat{\otimes}_{A} B_{p}$, where $B_{i}=A\left\{T_{i 0}, \ldots, T_{i n_{i}}\right\} /\left(T_{i 0} \cdot \ldots \cdot T_{i n_{i}}-a_{i}\right), a_{i} \in A$ and $n_{i} \geq 1$. We construct as follows a continuous map $\bar{\delta}_{\varphi}:\left|E_{\varphi_{s}}\right| \rightarrow S(\mathfrak{Y} / \mathfrak{X})$ over $\delta$. First of all, the continuous map $\mathfrak{Y}_{\eta} \rightarrow \mathfrak{X}_{\eta} \times[0,1]^{[\mathbf{n}]}$ that takes a point $y$ to $\left(\varphi(y) ;\left|T_{00}(y), \ldots,\right| T_{p n_{p}}(y) \mid\right)$ identifies $S(\mathfrak{Y} / \mathfrak{X})$ with the closed subset

$$
S_{\varphi}=\left\{\left(x ; \mathbf{r}_{0}, \ldots, \mathbf{r}_{p}\right) \in \mathfrak{X}_{\eta} \times[0,1]^{[\mathbf{n}]}\left|r_{i 0} \cdot \ldots \cdot r_{i n_{i}}=\right| a_{i}(x) \mid, 0 \leq i \leq p\right\}
$$

(see Step 1 from §5). Furthermore, one has

$$
\left|E_{\varphi_{s}}\right|=\operatorname{Coker}\left(\coprod \Sigma^{\mathbf{d}} \times\left|\mathbf{C}\left(\mathfrak{Y}_{s, \bar{\alpha}}\right)\right| \stackrel{\rightarrow}{\rightarrow} \coprod \Sigma^{\mathbf{c}} \times\left|\mathbf{C}\left(\mathfrak{Y}_{s, \bar{\alpha}}\right)\right|\right),
$$

where the first and the second coproducts are taken over the categories $N_{1}(\boldsymbol{\Lambda} / E)$ and $N_{0}(\boldsymbol{\Lambda} / E)$, respectively, and, given $(\boldsymbol{\Lambda}[\mathbf{d}] \stackrel{\gamma}{\rightarrow} \boldsymbol{\Lambda}[\mathbf{c}] \stackrel{\alpha}{\rightarrow} E)$ in $N_{1}(\boldsymbol{\Lambda} / E)$, the upper map $d_{0}$ acts as $(|\gamma|, 1)$, and the lower map $d_{1}$ acts as 
$\left(1,\left|D_{\mathscr{Q}}(\gamma)\right|\right)$. Thus, we have to construct a compatible system of continuous maps $\delta_{\alpha}: \Sigma^{\mathbf{c}} \times\left|\mathbf{C}\left(\mathfrak{Y}_{s, \bar{\alpha}}\right)\right| \rightarrow S_{\varphi}$. Let $I=\left\{i \in[p] \mid a_{i}(\bar{\alpha})=0\right\}$. Then $\left|\mathbf{C}\left(\mathfrak{Y}_{s, \bar{\alpha}}\right)\right|$ is identified with $\Sigma^{\mathbf{n}_{I}}$. If $\mathbf{u} \in \Sigma^{\mathbf{c}}$ and $\mathbf{v}=\left(\mathbf{v}_{i}\right)_{i \in I} \in\left|\mathbf{C}\left(\mathfrak{Y}_{s, \bar{\alpha}}\right)\right|$, then $\widetilde{\delta}_{\alpha}(\mathbf{u}, \mathbf{v})=\left(x ; \mathbf{r}_{0}, \ldots, \mathbf{r}_{p}\right) \in S_{\varphi}$, where $x$ is the image of $\mathbf{u}$ in $\mathfrak{X}_{\eta}$, and $\mathbf{r}_{i}=\phi_{n_{i}}\left(\mathbf{v}_{i},\left|a_{i}(x)\right|\right)$ for $i \in I$ and $\mathbf{r}_{i}=(1, \ldots, 1)$ for $i \notin I$. That the maps $\widetilde{\delta}_{\alpha}$ are continuous is evident, and that they are compatible easily follows from the construction. We remark that the map $\bar{\delta}_{\varphi}:\left|E_{\varphi_{s}}\right| \rightarrow \mathfrak{Y}_{\eta}$ possesses the same property (a) as $\delta$, namely, for each $\left(\boldsymbol{\Lambda}[\mathbf{c}] \stackrel{\alpha}{\rightarrow} E_{\varphi_{s}}\right) \in$ $O\left(E_{\varphi_{s}}\right)$ the image of $\stackrel{\circ}{\Sigma}^{\mathbf{c}}$ under the corresponding map to $\mathfrak{Y}_{\eta}$ is contained in $\pi^{-1}(\bar{\alpha})$. If the polysimplicial set $E$ is interiorly free and the map $\delta$ induces a homeomorphism of $|E|$ with its image in $\mathfrak{X}_{\eta}$ then, by Corollary 6.6, $\bar{\delta}_{\varphi}$ induces a homeomorphism of $\left|E_{\varphi_{s}}\right|$ with its image in $\mathfrak{Y}_{\eta}$. Finally, given an open formal subscheme $\mathfrak{Y}^{\prime} \subset \mathfrak{Y}$, the map $\bar{\delta}_{\varphi}$ induces a continuous map $\bar{\delta}_{\varphi^{\prime}}:\left|E_{\varphi_{s}^{\prime}}\right| \rightarrow S\left(\mathfrak{Y}^{\prime} / \mathfrak{X}\right)$, where $\varphi^{\prime}$ is the morphism $\mathfrak{Y}^{\prime} \rightarrow \mathfrak{X}$. If $E$ is interiorly free and $\delta$ induces a homeomorphism of $|E|$ with its image in $\mathfrak{X}_{\eta}$, then $\bar{\delta}_{\varphi^{\prime}}$ induces a homeomorphism of $\left|E_{\varphi_{s}^{\prime}}\right|$ with its image in $\mathfrak{Y}_{\eta}^{\prime}$.

Step 3. In the situation of Step 2, assume we are given a strictly polystable morphism $\psi: \mathfrak{Z} \rightarrow \mathfrak{X}$ that factors through an étale morphism $f: \mathfrak{Z} \rightarrow \mathfrak{Y}$ and such that, for each point $\mathbf{x} \in \operatorname{str}\left(\mathfrak{X}_{s}\right)$, the fibre $\mathfrak{Y}_{s, \mathbf{x}}$ is elementary. (For example, by Corollary 2.12, the latter is true if $\mathfrak{Z}_{s}$ is elementary.) It follows that if $\mathfrak{Y}^{\prime}$ is the image of $\psi$ in $\mathfrak{Y}$ and $\varphi^{\prime}$ is the morphism $\mathfrak{Y}^{\prime} \rightarrow \mathfrak{X}$, then $E_{\psi_{s}} \stackrel{\sim}{\rightarrow} E_{\varphi^{\prime}}$. The property (b) of $\delta$ implies that, for each point $x \in \operatorname{Im}(\delta)$, one has $\operatorname{str}\left(\mathfrak{Z}_{x, s}\right) \stackrel{\sim}{\rightarrow} \operatorname{str}\left(\mathfrak{Z}_{s, \pi(x)}\right)$ and, therefore, $S\left(\mathfrak{Z}_{x}\right)=S(\mathfrak{Z} / \mathfrak{X}) \cap \varphi^{-1}(x) \stackrel{\sim}{\rightarrow} S\left(\mathfrak{Y}_{x}^{\prime}\right)=S\left(\mathfrak{Y}^{\prime} / \mathfrak{X}\right) \cap \varphi^{\prime-1}(x)$. Thus, the map $\bar{\delta}_{\varphi^{\prime}}$ from Step 2 induces a continuous map $\bar{\delta}_{\psi}:\left|E_{\psi_{s}}\right| \rightarrow S(\mathfrak{Z} / \mathfrak{X})$ that possesses the same property (a) as $\delta$ and such that, if $E$ is interiorly free and $\delta$ induces a homeomorphism of $|E|$ with its image in $\mathfrak{X}_{\eta}$, then $\bar{\delta}_{\psi}$ induces a homeomorphism of $\left|E_{\psi_{s}}\right|$ with its image in $\mathfrak{Z}_{\eta}$. We claim that the map $\bar{\delta}_{\psi}:\left|E_{\psi_{s}}\right| \rightarrow S(\mathfrak{Z} / \mathfrak{X})$ does not depend on the decomposition $\psi=\varphi \circ f$. It suffices to prove that, given $(\boldsymbol{\Lambda}[\mathbf{c}] \stackrel{\alpha}{\rightarrow} E)$ in $N_{0}(\boldsymbol{\Lambda} / E)$ and a point $\mathbf{u} \in \stackrel{\circ}{\Sigma}^{\mathbf{c}}$, the homeomorphism $\left|\mathbf{C}\left(\mathfrak{Z}_{s, \bar{\alpha}}\right)\right| \stackrel{\sim}{\rightarrow} S\left(\mathfrak{Z}_{x}\right)$, induced by the homeomorphism $\left|\mathbf{C}\left(\mathfrak{Y}_{s, \bar{\alpha}}\right)\right| \stackrel{\sim}{\rightarrow} S\left(\mathfrak{Y}_{x}\right): \mathbf{v} \mapsto \widetilde{\delta}_{\bar{\alpha}}(\mathbf{u}, \mathbf{v})$, does not depend on the decomposition $\psi=\varphi \circ f$. (Here $x$ is the image of $\mathbf{u}$ in $\mathfrak{X}_{\eta}$.) To verify the latter, we can replace $\mathfrak{X}$ by $\operatorname{Spf}\left(\mathcal{H}(x)^{\circ}\right)$, and $\mathfrak{Y}$ and $\mathfrak{Z}$ by $\mathfrak{Y}_{x}$ and $\mathfrak{Z}_{x}$, respectively, and so we may assume that $\mathfrak{X}=\operatorname{Spf}\left(k^{\circ}\right)$. If $I=\left\{i \in[p]|| a_{i} \mid<1\right\}$, then $|\mathbf{C}(\mathfrak{Y})|$ is identified with $\Sigma^{\mathbf{n}_{I}}$, and the homeomorphism $\bar{\delta}:\left|\mathbf{C}\left(\mathfrak{Z}_{s}\right)\right| \stackrel{\sim}{\rightarrow} S(\mathfrak{Z})$ is induced by the injective map $\mu:\left|\mathbf{C}\left(\mathfrak{Y}_{s}\right)\right| \rightarrow[0,1]^{\mathbf{n}]}$ defined by $\mu(\mathbf{v})=\left(\mathbf{r}_{0}, \ldots, \mathbf{r}_{p}\right)$, where $\mathbf{v}=\left(\mathbf{v}_{i}\right)_{i \in I} \in \Sigma^{\mathbf{n}_{I}}, \mathbf{r}_{i}=\phi_{n_{i}}\left(\mathbf{v}_{i},\left|a_{i}\right|\right)$ for $i \in I$ and $\mathbf{r}_{i}=(1, \ldots, 1)$ for $i \notin I$. By Lemma 4.1, to show that $\bar{\delta}$ does not depend on the choice of $f$ it suffices to check that the sheaf of monoids on $\left|\mathbf{C}\left(\mathfrak{Z}_{s}\right)\right|$, generated by the restrictions of the coordinate functions on $[0,1]^{\mathrm{n}]}$ with respect to the 
injective map $\left|\mathbf{C}\left(\mathfrak{Z}_{s}\right)\right| \rightarrow\left|\mathbf{C}\left(\mathfrak{Y}_{s}\right)\right| \stackrel{\mu}{\rightarrow}[0,1]^{\mathbf{n}]}$, does not depend on $f$. But this is an easy consequence of Proposition 4.3.

The remaining part of the proof follows Steps 6-8 from the proof of Theorem 7.2.

Step 4. Assume we are given a morphism $g: \mathfrak{X}^{\prime}=\operatorname{Spf}\left(A^{\prime}\right) \rightarrow \mathfrak{X}$ in $\mathcal{F} s c h$, where $\mathfrak{X}^{\prime}$ is strictly pluri-nodal and is provided with a polysimplicial set $E^{\prime}$, a map of partially ordered sets $O\left(E^{\prime}\right) \rightarrow \operatorname{str}\left(\mathfrak{X}_{s}^{\prime}\right)$ and a continuous map $\delta^{\prime}:\left|E^{\prime}\right| \rightarrow \mathfrak{X}_{\eta}^{\prime}$ possessing the properties (a) and (b) from Step 2 and compatible with the corresponding objects for $\mathfrak{X}$. Furthermore, assume we are given a strictly poly-stable morphism $\psi^{\prime}: \mathfrak{Z}^{\prime} \rightarrow \mathfrak{X}^{\prime}$, possessing the same properties from Step 3 as the morphism $\psi$, and a commutative diagram in $\mathcal{F}$ sch

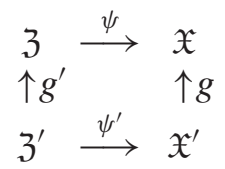

such that the morphism $\mathfrak{Z}^{\prime} \rightarrow \mathfrak{Z} \times \mathfrak{X} \mathfrak{X}^{\prime}$ is étale. Then Step 3 implies that the following diagram of continuous maps is commutative

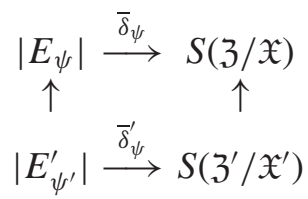

Step 5. Let us return to the theorem. Assume that there is an isomorphism of functors $\left|\mathbf{C}^{l-1}\right| \stackrel{\sim}{\rightarrow} S^{l-1}$ possessing the necessary property. For a polystable fibration $\underline{\mathfrak{X}}=\left(\mathfrak{X}_{l} \stackrel{f_{l-1}}{\rightarrow} \cdots \stackrel{f_{1}}{\rightarrow} \mathfrak{X}_{1}\right)$, we construct as follows an étale morphism $\varphi:{\underline{\mathfrak{X}^{\prime}}}^{\rightarrow} \underline{\mathfrak{X}}$ such that $\mathfrak{X}^{\prime}$ is a strictly poly-stable fibration and the morphism $\mathfrak{X}_{l}^{\prime} \rightarrow \mathfrak{X}_{l}$ is surjective. First of all, let $\mathfrak{Y} \rightarrow \underline{\mathfrak{X}}$ be an étale morphism from a strictly poly-stable fibration $\mathfrak{Y}$ such that the morphism $\mathfrak{Y}_{l} \rightarrow \mathfrak{X}_{l}$ is surjective. Furthermore, for each point $\mathbf{y} \in \mathfrak{Y}_{l, s}$, take an open affine neighborhood $\mathfrak{U}$ of the point $f_{l-1, s}(\mathbf{y})$ and an open affine neighborhood $\mathfrak{V}$ of the point $\mathbf{y}$ over $\mathfrak{U}$ with elementary closed fibre $\mathfrak{V}_{s}$. We set $\underline{\mathfrak{X}}^{\prime}=\left(\mathfrak{X}_{l}^{\prime} \rightarrow \mathfrak{X}_{l-1}^{\prime} \rightarrow \mathfrak{Y}_{l-2} \rightarrow \cdots \rightarrow \mathfrak{Y}_{1}\right)$, where $\mathfrak{X}_{l}^{\prime}$ is a disjoint union of $\mathfrak{V}$ 's of the above form such that the induced morphism $\mathfrak{X}_{l}^{\prime} \rightarrow \mathfrak{Y}_{l}$ is surjective, and $\mathfrak{X}_{l-1}^{\prime}$ is a disjoint union of the corresponding $\mathfrak{U}$ 's.

Step 6. For a poly-stable fibration $\mathfrak{X}$, take étale morphisms $\underline{\mathfrak{X}}^{\prime} \rightarrow \underline{\mathfrak{X}}$ and $\underline{\mathfrak{X}}^{\prime \prime} \rightarrow \underline{\mathfrak{X}}^{\prime} \times \underline{\underline{X}} \underline{\mathfrak{X}}^{\prime}$ as constructed in Step 5. By induction, the construction from Step 3 gives homeomorphisms $\left|\mathbf{C}\left(\underline{\mathfrak{X}}_{s}^{\prime}\right)\right| \stackrel{\sim}{\rightarrow} S\left(\underline{\mathfrak{X}}^{\prime}\right)$ and $\left|\mathbf{C}\left(\underline{\mathfrak{X}}_{s}^{\prime \prime}\right)\right| \stackrel{\sim}{\rightarrow} S\left(\underline{\mathfrak{X}}^{\prime \prime}\right)$ which are compatible, by Step 4 . Since $\operatorname{Coker}\left(\mathbf{C}\left(\underline{\mathfrak{X}}_{s}^{\prime \prime}\right) \rightarrow \mathbf{C}\left(\underline{\mathfrak{X}}_{s}^{\prime}\right)\right) \stackrel{\sim}{\rightarrow} \mathbf{C}\left(\underline{\mathfrak{X}}_{s}\right)$ and $\operatorname{Coker}\left(S\left(\underline{\mathfrak{X}}^{\prime \prime}\right) \stackrel{\rightarrow}{\rightarrow} S\left(\left(\underline{\mathfrak{X}}^{\prime}\right)\right) \stackrel{\sim}{\rightarrow} S(\underline{\mathfrak{X}})\right.$, we get the required homeomorphism $|\mathbf{C}(\underline{\mathfrak{X}})| \stackrel{\sim}{\rightarrow} S(\underline{\mathfrak{X}})$. That in this way we get an isomorphism of functors $\left|\mathbf{C}^{l}\right| \stackrel{\sim}{\rightarrow} S^{l}$ easily follows from the construction. 
The first corollary is a consequence of Theorem 8.1.

Corollary 8.4. Given a poly-stable fibration $\mathfrak{x}$ of length l over $k^{\circ}$, a locally closed strata subset $\mathcal{X} \subset \mathfrak{X}_{l, s}$, and an open subset $X \subset \pi^{-1}(\mathcal{X})$ which is the intersection of $\pi^{-1}(\mathcal{X})$ with a family of Zariski open subsets of $\mathfrak{X}_{\eta}$, the homotopy $\Phi^{l}$ induces a strong deformation retraction of $X$ to $S(\mathfrak{X}) \cap X$. If $X$ is dense in $\pi^{-1}(\mathcal{X})$ and contains its non-normality locus, then $S(\underline{\mathfrak{X}}) \cap X=$ $S(\underline{\mathfrak{X}})$.

Proof. We may assume that $\mathfrak{X}_{l}=\operatorname{Spf}(A)$ is affine. Let $\mathcal{U}$ be a Zariski open subset of $\mathfrak{X}_{\eta}$, and let $f_{1}, \ldots, f_{n}$ be generators of the ideal of $A \otimes_{k^{\circ}} k$ that corresponds to the complement of $\mathcal{U}$. If $x \in \mathcal{U}$, then $f_{i}(x) \neq 0$ for some $1 \leq i \leq n$. Since $x \leq x_{t}$, it follows that $f_{i}\left(x_{t}\right) \neq 0$, i.e., $x_{t} \in \mathcal{U}$, for all $t \in[0, l]$. This implies the first statement. The second statement follows from the fact that the local ring of a normal point from $S(\underline{X})$ is a field.

The following corollary will be used in the next two sections.

Corollary 8.5. Suppose we are given a finite normal extension $k^{\prime}$ of $k$, a poly-stable fibration $\underline{\mathfrak{X}}$ over $k^{\prime \circ}$ of length $l$ with normal generic fibre $\mathfrak{X}_{l, \eta}$, an action of a finite group $G$ on $\underline{x}$ over $k^{\circ}, a G$-invariant locally closed strata subset $\mathcal{X} \subset \mathfrak{X}_{l, s}$, and a dense $G$-invariant Zariski open subset $\mathcal{U} \subset \mathfrak{X}_{l, \eta}$. Let $X$ be the quotient space $G \backslash\left(\pi^{-1}(X) \cap \mathcal{U}\right)$. Then

(i) there exists a strong deformation retraction of $X$ to a closed subset homeomorphic to $G \backslash|\mathbf{C}(\mathcal{X})|$;

(ii) for any abelian group $M$ there are canonical isomorphisms

$$
H^{q}(|X|, M) \stackrel{\sim}{\rightarrow} H_{\text {sing }}^{q}(|X|, M) .
$$

Notice that the quotient space $G \backslash|\mathbf{C}(\mathcal{X})|$ coincides with the locally closed subset of $\left|G \backslash \mathbf{C}\left(\underline{\mathfrak{x}}_{s}\right)\right|$ which is a union of the cells that correspond to the strata of $\mathfrak{X}_{l, s}$ contained in $\mathcal{X}$. In particular, Corollary 3.11 implies that the space $G \backslash|\mathbf{C}(\mathcal{X})|$ is locally contractible. Furthermore, here $H^{q}(|X|, M)$ denote the usual cohomology groups of the underlying topological space $|X|$ of $X$ with coefficients in the constant sheaf generated by $M$. They coincide with the cohomology groups of the associated rigid analytic space (see [Ber2, §1.6]). Finally, $H_{\text {sing }}^{q}(|X|, M)$ denote the singular cohomology groups of $|X|$ with coefficients in $M$.

Proof. (i) By Corollary 8.4, the homotopy $\Phi^{l}$ induces a strong deformation retraction of $\pi^{-1}(\mathcal{X}) \cap \mathcal{U}$ to $S(\underline{X}) \cap \pi^{-1}(\mathcal{X})$ and, by Theorem 8.2, there is a canonical homeomorphism between the latter space and $|\mathbf{C}(\mathcal{X})|$. Since the homotopy $\Phi^{l}$ and the above homeomorphism commute with the action of $G$, the statement follows.

(ii) Since $X$ is paracompact, the groups $H^{q}(|X|, M)$ coincide with the Čech cohomology groups of $|X|$. By [Spa, Ch. 6], the latter ones coincide with the Alexander cohomology groups of $|X|$ which, in their turn, 
satisfy the homotopy axiom and, therefore, coincide with the Alexander cohomology groups of $G \backslash|\mathbf{C}(\mathcal{X})|$. Since the space $G \backslash|\mathbf{C}(\mathcal{X})|$ is paracompact and locally contractible, its Alexander cohomology groups coincide with $H_{\text {sing }}^{q}(G \backslash|\mathbf{C}(\mathcal{X})|, M)$. It remains to use the fact that the singular cohomology groups also satisfy the homotopy axiom.

Corollary 8.6. Let $\underline{\mathfrak{x}}$ be a poly-stable fibration of formal schemes over $k^{\circ}$ of length $l$ provided with an action of a group $\Gamma$, and assume that $\mathfrak{X}_{l}$ is separated and the stabilizer in $\Gamma$ of each of its open affine subscheme is finite. Then there exists a strong deformation retraction of the quotient space $\Gamma \backslash \mathfrak{X}_{l, \eta}$ to a closed subset homeomorphic to $\left|\Gamma \backslash \mathbf{C}\left(\underline{\mathfrak{x}}_{s}\right)\right|$.

In the situation of Corollary 8.6, the quotient space $\Gamma \backslash \mathfrak{X}_{l, \eta}$ exists by [Ber5, Lemma 4.2].

Corollary 8.7. Let $\underline{\mathfrak{x}}$ be a poly-stable fibration oflengthl over $k^{\circ}, \mathcal{X}$ a locally closed strata subset of $\mathfrak{X}_{l, s}$, and $X$ a dense open subset of $\pi^{-1}(\mathcal{X})$ which is the intersection of $\pi^{-1}(\mathcal{X})$ with a family of Zariski open subsets of $\mathfrak{X}_{l, \eta}$.

(i) If $\mathcal{X}$ is quasi-compact, there exists a finite unramified extension $k^{\prime}$ of $k$ such that, for any non-Archimedean field $K$ over $k^{\prime}$ and any subset $Y$ of a Hausdorff K-analytic space $Z$, the canonical map $|X \times Y| \rightarrow\left|X \widehat{\otimes} k^{\prime}\right| \times|Y|$ is a homotopy equivalence.

(ii) If $\underline{\mathfrak{X}}$ is a strictly poly-stable fibration and all of the strata of $\mathfrak{X}_{l, s}$ in $\mathcal{X}$ are geometrically irreducible, then the conclusion of $(i)$ is true for $k^{\prime}=k$.

Here and later (in $\S 10) X \times Y$ denotes the preimage of $Y$ under the canonical projection $X \times Z \rightarrow Z$. The sets $Y$ and $X \times Y$ are provided with the topology induced from $Z$ and $X \times Z$, respectively.

Proof. First of all, we claim that the map $\Psi:\left|\mathfrak{X}_{l, \eta} \times Z\right| \times[0, l] \rightarrow\left|\mathfrak{X}_{l, \eta} \times Z\right|$, which coincides with the map $\Phi^{l}$ from Theorem 8.1 on each fibre of the projection $\mathfrak{X}_{l, \eta} \times Z \rightarrow Z$, is continuous. Indeed, for this it suffices to consider the case when $Z$ is $K$-affinoid. In this case $Z$ is a closed analytic subset of a closed polydisc which, in its turn, is an affinoid domain in the projective space $\mathbf{P}^{n}$. The latter is the generic fibre of the formal projective space $\mathfrak{P}^{n}$ over $K^{\circ}$ and, therefore, the claim follows from the fact that the map $\Phi_{\mathfrak{P}^{n}}^{l}$ is continuous. By Corollary 8.4, the map $\Psi$ induces a strong deformation retraction of $X \times Y$ to a closed subset $V$ whose fibre at a point $y \in Y$ under the projection $V \rightarrow Y$ is $S\left(\underline{x} \widehat{\otimes} \mathscr{H}(y)^{\circ}\right) \cap(X \widehat{\otimes} \mathscr{H}(y))$.

In the situation of (ii), one has $|\mathbf{C}(\mathcal{X} \otimes \widetilde{\mathscr{H}(y)})| \stackrel{\sim}{\rightarrow}|\mathbf{C}(\mathcal{X})|$ and, therefore, $S\left(\underline{\mathfrak{X}} \widehat{\otimes} \mathscr{H}(y)^{\circ}\right) \cap \pi^{-1}(\mathcal{X} \otimes \widetilde{\mathscr{H}(y)}) \stackrel{\sim}{\rightarrow} S(\underline{\mathfrak{X}}) \cap \pi^{-1}(\mathcal{X})$. It follows that $S\left(\underline{\mathfrak{x}} \widehat{\otimes} \mathscr{H}(y)^{\circ}\right) \cap(X \widehat{\otimes} \mathscr{H}(y)) \stackrel{\sim}{\rightarrow} S(\underline{\mathfrak{X}}) \cap X$. Thus, the map $|X \times Y| \rightarrow|X| \times|Y|$ induces a homeomorphism $V \stackrel{\sim}{\rightarrow}(S(\underline{\mathfrak{X}}) \cap X) \times|Y|$, and the required statement follows from the fact that $X$ is homotopy equivalent to $S(\underline{\mathfrak{X}}) \cap X$.

In the situation of (i), let $\underline{\mathfrak{X}}^{\prime} \rightarrow \underline{\mathfrak{X}}$ be a surjective étale morphism from a strictly poly-stable fibration $\underline{\mathfrak{X}}^{\prime}$ such that the preimage $\mathcal{X}^{\prime}$ of $\mathcal{X}$ in $\mathfrak{X}_{l, s}^{\prime}$ is 
quasi-compact. Then the preimage $\mathcal{X}^{\prime \prime}$ of $\mathcal{X}$ in $\mathfrak{X}_{l, s}^{\prime \prime}$, where $\underline{\mathfrak{X}}^{\prime \prime}=\underline{\mathfrak{X}}^{\prime} \times \mathfrak{X} \underline{\mathfrak{X}}^{\prime}$, is also quasi-compact. We can replace $k$ by a finite unramified extension and assume that all of the strata in $X^{\prime}$ and $X^{\prime \prime}$ are geometrically irreducible. If $X^{\prime}$ and $X^{\prime \prime}$ (resp. $V^{\prime}$ and $V^{\prime \prime}$ ) are the preimages of $X$ (resp. $V$ ) in $\mathfrak{X}_{l, \eta}^{\prime}$ and $\mathfrak{X}_{l, \eta}^{\prime \prime}$, respectively, then, by (ii), $V^{\prime} \stackrel{\sim}{\rightarrow}\left(S\left(\underline{\mathfrak{X}}^{\prime}\right) \cap X^{\prime}\right) \times|Y|$ and $V^{\prime \prime} \stackrel{\sim}{\rightarrow}\left(S\left(\underline{\mathfrak{X}}^{\prime \prime}\right) \cap X^{\prime \prime}\right) \times$ $|Y|$. It follows that $V \stackrel{\sim}{\rightarrow}(S(\underline{\mathfrak{X}}) \cap X) \times|Y|$, i.e., (i) is also true.

Remark 8.8. Let $\mathfrak{X}$ be a pluri-nodal formal scheme for which there exist a poly-stable fibration $\underline{\mathfrak{X}}=\left(\mathfrak{X}_{l} \rightarrow \cdots \rightarrow \mathfrak{X}_{1}\right)$ with $\mathfrak{X}_{l}=\mathfrak{X}$. It is easy to see that the polysimplicial set $\mathbf{C}(\underline{\mathfrak{X}})$ and the homotopy $\Phi^{l}: \mathfrak{X}_{\eta} \times[0, l] \rightarrow \mathfrak{X}_{\eta}$ depend on the representation of $\mathfrak{X}$ in the above form. On the other hand, it is very likely that the skeleton $S(\underline{\mathfrak{X}})$ depends only on $\mathfrak{X}$.

\section{Local contractibility of smooth analytic spaces}

In this section the valuation on the ground field $k$ is assumed to be nontrivial, and all $k$-analytic spaces considered are strictly $k$-analytic (see [Ber2, §1]). The assumption on nontriviality of the valuation is used through the fact, which is implied by it, that each point of a strictly $k$-analytic space has a fundamental system of neighborhoods formed by compact strictly $k$-analytic domains. We say that a $k$-analytic space $X$ is locally embeddable in a smooth space if each point of $X$ has an open neighborhood isomorphic to a strictly $k$-analytic domain in a smooth $k$-analytic space. We remark that such a space is automatically strictly $k$-analytic. We also remark that any $k$-analytic space that admits an étale morphism to a space with the above property possesses the same property (this follows from [Ber2, Theorem 3.4.1]).

Theorem 9.1. Any k-analytic space locally embeddable in a smooth space is locally contractible.

Before proving the theorem, we establish a fact that contains everything we need from results of de Jong. (It will also be used in the next section.) The point is in inclusion the case of non-discrete valuation on the ground field $k$. Since the only property of the field $k$ we need is that it is the fraction field of a Henselian valuation ring, the following lemma is proven in a slightly more general setting.

Lemma 9.2. Let $A$ be a Henselian valuation ring, and let $\mathcal{X}$ be an integral scheme proper finitely presented and flat over $A$ and with geometrically irreducible generic fibre of dimension $l \geq 1$. Then there exist the following objects:

(a) a finite normal extension of the fraction field of A with the ring of integers $A^{\prime}$ (it is also a Henselian valuation ring);

(b) a poly-stable fibration $\underline{X}^{\prime}=\left(X_{l}^{\prime} \stackrel{f_{l-1}}{\rightarrow} \ldots \stackrel{f_{1}}{\rightarrow} X_{1}^{\prime} \stackrel{f_{0}}{\rightarrow} X_{0}^{\prime}=\right.$ $\operatorname{Spec}\left(A^{\prime}\right)$ ), where all morphisms $f_{i}$ are projective of dimension one and have smooth geometrically irreducible generic fibres; 
(c) an action of a finite group $G$ on $\underline{\mathcal{X}}^{\prime}$ over A;

(d) a commutative diagram

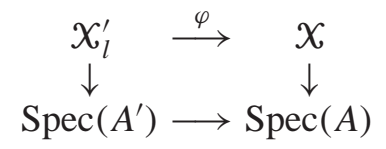

where $\varphi$ is a dominant G-equivariant morphism that induces a proper generically finite morphism $X_{l, \eta}^{\prime} \rightarrow X_{\eta}$ and such that the field $R\left(\mathcal{X}_{l}^{\prime}\right)^{G}$ is purely inseparable over $R(\mathcal{X})$.

Here $R(\mathcal{X})$ denotes the field of rational functions on $\mathcal{X}$. Notice, that in the case, when the valuation on $A$ is not discrete of rank one, the ring $A^{\prime}$ is not necessarily finitely generated over $A$ and, therefore, one cannot say that the morphism $\varphi$ is proper.

Proof. By [EGAIV, §8], there exists a flat (and therefore dominant) morphism $\alpha: \mathcal{Y} \rightarrow \mathcal{T}$ with geometrically irreducible generic fibre between integral schemes of finite type over $\operatorname{Spec}(\mathbf{Z})$ such that the morphism $X \rightarrow$ $\operatorname{Spec}(A)$ is the base change of $\alpha$ under a morphism $\delta=\operatorname{Spec}(A) \rightarrow \mathcal{T}$ with the property that the homomorphism $\mathcal{O}_{\mathcal{T}, t} \rightarrow A$ is injective, where $t$ is the image of the closed point of $\delta$ in $\mathcal{T}$. By Theorem 5.9 from [deJ3], there exist

(1) a Galois alteration $\left(\mathcal{T}^{\prime}, G^{\prime}\right)$ of $(\mathcal{T},\{1\})$, i.e., an integral scheme $\mathcal{T}^{\prime}$ provided with an action of a finite group $G^{\prime}$ and a dominant $G^{\prime}$-equivariant proper and generically finite morphism $\psi: \mathcal{T}^{\prime} \rightarrow \mathcal{T}$ such that the field $R\left(\mathcal{T}^{\prime}\right)^{G^{\prime}}$ is purely inseparable over $R(\mathcal{T})$;

(2) a $G^{\prime}$-pluri nodal fibration ${\underline{y^{\prime}}}^{\prime}=\left(\mathcal{y}_{l}^{\prime} \stackrel{h_{l-1}}{\rightarrow} \cdots \stackrel{h_{1}}{\rightarrow} y_{1}^{\prime} \stackrel{h_{0}}{\rightarrow} y_{0}^{\prime}=\mathcal{T}^{\prime},\left\{\sigma_{i j}\right\}, Z_{0}\right)$ over $\mathcal{T}^{\prime}$, i.e., each $h_{i}$ is a projective semi-stable curve provided with an action of $G^{\prime}, \mathfrak{Z}_{0}$ is a $G^{\prime}$-stable proper closed subset of $\mathcal{T}^{\prime}$, and $\left\{\sigma_{i j}\right\}_{1 \leq j \leq n_{i}}$ is a $G^{\prime}$-equivariant system of sections of $h_{i}$ into the smooth locus of $h_{i}$, such that $h_{i}$ is smooth over $Y_{i}^{\prime} \backslash Z_{i}$ where, for $i \geq 1$, $\mathcal{Z}_{i}=\cup_{j=1}^{n_{i-1}} \sigma_{i-1, j}\left(\mathcal{Y}_{i-1}^{\prime}\right) \cup h_{i-1}^{-1}\left(\mathcal{Z}_{i-1}\right)$;

(3) a commutative diagram

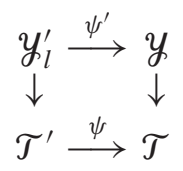

such that $\left(\mathcal{Y}_{l}^{\prime}, G^{\prime}\right)$ is a Galois alteration of $(\mathcal{Y},\{1\})$.

Let $K$ be the fraction field of $A$, and let $K^{\prime \prime}$ be the quotient algebra of the tensor product $R\left(\mathcal{T}^{\prime}\right) \otimes_{R(\mathcal{T})} K$ by its radical. Then $K^{\prime \prime}$ is a finite direct product of finite normal extensions of $K$ provided with an action of $G^{\prime}$ over $K$. Let $K^{\prime}$ be a factor of $K^{\prime \prime}, G$ its stabilizer in $G^{\prime}$, and $A^{\prime}$ the integral closure of $A$ in $K^{\prime}$. By the valuative criterion of properness [EGAIV , 7.3.8], there exists a unique lift $\operatorname{Spec}\left(A^{\prime}\right) \rightarrow \mathcal{T}^{\prime}$ of the morphism $\operatorname{Spec}\left(K^{\prime}\right) \rightarrow \mathcal{T}^{\prime}$ 
over the morphism $\operatorname{Spec}\left(A^{\prime}\right) \rightarrow \mathcal{T}$, and the morphism $\operatorname{Spec}\left(A^{\prime}\right) \rightarrow \mathcal{T}^{\prime}$ is $G$-equivariant. Thus, we get a poly-stable fibration $\underline{\mathcal{X}}^{\prime}={\underline{\mathcal{Y}^{\prime}}}^{\prime} \otimes_{\mathcal{T}^{\prime}} \operatorname{Spec}\left(A^{\prime}\right)$ provided with an action of the group $G$ and a morphism $\varphi: \mathcal{X}_{l}^{\prime} \rightarrow \mathcal{X}$ over the morphism $\operatorname{Spec}\left(A^{\prime}\right) \rightarrow \operatorname{Spec}(A)$. We claim that all of the necessary conditions are satisfied.

First of all, each of the morphisms $f_{i}: X_{i+1}^{\prime} \rightarrow X_{i}^{\prime}$ is projective of dimension one and poly-stable because it is a base change of the similar morphism $h_{i}$. If $t^{\prime}$ is the image of the closed point of $\operatorname{Spec}\left(A^{\prime}\right)$ in $\mathcal{T}^{\prime}$, then the homomorphism $\mathcal{O}_{\mathcal{T}^{\prime}, t^{\prime}} \rightarrow A^{\prime}$ is injective because its composition with the injection $A^{\prime} \rightarrow K^{\prime}$ coincides with the injection $\mathcal{O}_{\mathcal{T}^{\prime}, t^{\prime}} \rightarrow R\left(\mathcal{T}^{\prime}\right) \rightarrow K^{\prime}$. It follows that the image of the generic point of $\operatorname{Spec}\left(A^{\prime}\right)$ in $\mathcal{T}^{\prime}$ is the generic point of $\mathcal{T}^{\prime}$ and, therefore, the geometric fibre of each $f_{i}$ is a base change of the geometric fibre of $h_{i}$, i.e., it is smooth and connected. Furthermore, since the morphism $\psi^{\prime}: y_{l}^{\prime} \rightarrow y$ is proper and generically finite and the image of the generic point of $\operatorname{Spec}\left(A^{\prime}\right)$ is $\mathcal{T}^{\prime}$ is the generic point of $\mathcal{T}^{\prime}$, it follows that $\varphi$ induces a proper generically finite morphism $X_{l, \eta}^{\prime} \rightarrow X_{\eta}$. Finally, since $R\left(\mathcal{X}_{l}^{\prime}\right)$ is a factor of the quotient of $R\left(\mathcal{Y}_{l}^{\prime}\right) \otimes_{R(y)} R(\mathcal{X})$ by the radical, the following lemma (applied to $K_{0}=R(y), K_{0}^{\prime}=R\left(\mathcal{Y}_{l}^{\prime}\right)$, $K=R(\mathcal{X})$ and the action of $G^{\prime}$ on $\left.R\left(\mathcal{Y}_{l}^{\prime}\right)\right)$ implies that the field $R\left(\mathcal{X}_{l}^{\prime}\right)^{G}$ is purely inseparable over $R(\mathcal{X})$.

Lemma 9.3. Suppose we are given a field $K_{0}$, a finite normal extension $K_{0}^{\prime}$ of $K_{0}$ endowed with an action of a finite group $G^{\prime}$ over $K_{0}$ such that $K_{0}^{\prime G^{\prime}}$ is purely inseparable over $K_{0}$, and an extension $K$ of $K_{0}$. Let $K^{\prime \prime}$ be the quotient of $K_{0}^{\prime} \otimes_{K_{0}} K$ by the radical, $K^{\prime}$ a factor of $K^{\prime \prime}$, and $G$ the stabilizer of $K^{\prime}$ in $G^{\prime}$. Then the field $K^{\prime G}$ is purely inseparable over $K$.

Proof. Let $H=\operatorname{Im}\left(G^{\prime} \rightarrow \operatorname{Aut}\left(K_{0}^{\prime}\right)\right), R_{0}=K_{0}^{\prime H} \otimes_{K_{0}} K, \mathbf{r}_{0}$ the radical of $R_{0}$, and $L=R_{0} / \mathbf{r}_{0}$. Since $K_{0}^{\prime H}$ is purely inseparable over $K_{0}, L$ is a field purely inseparable over $K$. By the normal basis theorem [Lang, Ch. VIII, $\S 12], K_{0}^{\prime}$ is isomorphic to a free $K_{0}^{\prime H}[H]$-module of rank one. It follows that for $R:=K_{0}^{\prime} \otimes_{K_{0}} K=K_{0}^{\prime} \otimes_{K_{0}^{\prime}{ }^{H}} R_{0}$ one has $R^{H}=R_{0}$. Tensoring the exact sequence $0 \rightarrow \mathbf{r}_{0} \rightarrow R_{0} \rightarrow L \rightarrow 0$ with $K_{0}^{\prime}$ over $K_{0}^{\prime}{ }^{H}$, we get an exact sequence $0 \rightarrow K_{0}^{\prime} \otimes_{K_{0}^{\prime}{ }^{H}} \mathbf{r}_{0} \rightarrow R \rightarrow K_{0}^{\prime} \otimes_{K_{0}^{\prime}{ }^{H}} L \rightarrow 0$. Since $K_{0}^{\prime}$ is a finite separable extension of $K_{0}^{\prime H}, K_{0}^{\prime} \otimes_{K_{0}^{\prime}{ }^{H}} L$ is a finite separable algebra over $L$ and, therefore, $K_{0}^{\prime} \otimes_{K_{0}^{\prime}{ }^{H} \mathbf{r}_{0}}$ is the radical of $R$. It follows that $K^{\prime \prime}=K_{0}^{\prime} \otimes_{K_{0}^{\prime}{ }^{H}} L, K^{\prime \prime H}=L$ and, in particular, $G^{\prime}$ acts transitively on the set of direct factors of $K^{\prime \prime}$. The latter implies that there is an isomorphism of $G^{\prime}$-modules $K^{\prime \prime} \stackrel{\sim}{\rightarrow} \operatorname{Ind}_{G}^{G^{\prime}}\left(K^{\prime}\right)$. It follows that $K^{\prime G}=K^{\prime \prime} G^{\prime}=L$, i.e., $K^{\prime G}$ is purely inseparable over $K$.

Proof of Theorem 9.1. Since each point of a smooth $k$-analytic space has an open neighborhood isomorphic to an open subset of the analytification of 
a smooth affine scheme over $k$, we may assume that we are given a compact strictly $k$-analytic domain $X$ in $X^{\text {an }}$, where $X=\operatorname{Spec}(A)$ is a smooth irreducible affine scheme over $k$. Let $x$ be a point of $X$ and let $\mathbf{x}$ be the image of $x$ in $X$. There are the following two possibilities:

(a) $\mathbf{x}$ is not the generic point of $\mathcal{X}$;

(b) $\mathbf{x}$ is the generic point of $x$.

We prove local contractibility of $X$ at the point $x$ by induction. In the case (a) we'll reduce the statement to the case of smaller dimension, and in the case (b) we'll use Lemma 9.2 and Corollary 8.5(i).

Case (a). Step 1. One may assume that the field $k(\mathbf{x})=\mathcal{O}_{x, \mathbf{x}} / \mathbf{m}_{\mathbf{x}}$ is separable over $k$. Indeed, since $k(\mathbf{x})$ is finitely generated over $k$, we can find a finite purely inseparable extension $k^{\prime}$ of $k$ such that the field $k\left(\mathbf{x}^{\prime}\right)$ is separable over $k^{\prime}$, where $\mathbf{x}^{\prime}$ is the (unique) preimage of $\mathbf{x}$ in $\mathcal{X} \otimes k^{\prime}$. Since the map $X \widehat{\otimes} k^{\prime} \rightarrow X$ is a homeomorphism, we can replace $k$ by $k^{\prime}, X$ by $X \widehat{\otimes} k^{\prime}$ and $\mathcal{X}$ by $\mathcal{X} \otimes k^{\prime}$, and reduce the situation to the case when the field $k(\mathbf{x})$ is separable over $k$.

Step 2. There is an isomorphism of an open neighborhood of the point $x$ in $X$ onto $Y \times D(0, r)$ that takes $x$ to $Y \times\{0\}$, where $Y$ is also a strictly $k$-analytic domain in the analytification of a smooth scheme over $k$ and $D(0, r)$ is the open disc of radius $r>0$ in $\mathbf{A}^{1}$ with center at zero. By Step 1, the closure $X^{\prime}$ of $\mathbf{x}$ in $X$ is smooth at $\mathbf{x}$. It follows that we can shrink $X$ (and $X$ ) and find an étale morphism from $\mathcal{X}$ to the affine space $A^{d}$, where $d=\operatorname{dim}(\mathcal{X})=\operatorname{dim}(X)$, such that $\mathcal{X}^{\prime}$ is contained in the preimage $\mathcal{Y}$ of $A^{d-1}=\left\{\mathbf{y} \in A^{d} \mid T_{d}(\mathbf{y})=0\right\}$. Let $Y=X \cap y^{\text {an }}$. The above morphism and its composition with the projection to the first $d-1$ coordinates $A^{d} \rightarrow$ $A^{d-1}$ give rise to the following cartesian diagram of morphisms of strictly $k$-analytic spaces

$$
\begin{array}{ccc}
Z=X \times_{\mathbf{A}^{d-1}} & \stackrel{g}{\downarrow} & Y \\
X & & \downarrow \\
X & \mathbf{A}^{d-1}
\end{array}
$$

The canonical closed immersion $Y \rightarrow X$ induces a morphism $Y \rightarrow Z$ that takes the point $x$ to a point $z$. We claim that $f$ is étale and $g$ is smooth at the point $z$. Assume that the claim is true. Since $\mathscr{H}(x) \stackrel{\sim}{\rightarrow} \mathscr{H}(z)$, from $[\mathrm{Ber} 2$, Theorem 3.4.1], it follows that $f$ is a local isomorphism $z$. Then we can shrink $X$ and $Y$ so that there is a smooth morphism of pure dimension one $X \rightarrow Y$, whose composition with the canonical closed immersion $Y \rightarrow X$ is the identity on $Y$, and the required fact follows from [Ber2, Proposition 3.7.8].

Since $f$ (resp. $g$ ) is the restriction of the étale (resp. smooth) morphism $Z^{\prime}=X \times_{\mathbf{A}^{d-1}} y^{\text {an }} \rightarrow X$ (resp. $Z^{\prime \prime}=X^{\text {an }} \times_{\mathbf{A}^{d-1}} Y \rightarrow Y$ ) to the analytic domain $Z$, it suffices to verify that the point $z$ is contained in the topological interior of $Z$ in $Z^{\prime}$ (resp. $Z^{\prime \prime}$ ). If $X=X_{1} \cup \cdots \cup X_{n}$ is a covering of $X$ by affinoid domains, then it suffices to check that $z$ is contained in the topological interior of $Z_{i}$ in $Z_{i}^{\prime}\left(\right.$ resp. $Z_{i}^{\prime \prime}$ ) for each $i$ with $z \in X_{i}$, where $Z_{i}=$ 
$X_{i} \times_{\mathbf{A}^{d-1}} Y_{i}, Y_{i}=X_{i} \cap \mathcal{Y}^{\text {an }}$ and $Z_{i}^{\prime}=X_{i} \times_{\mathbf{A}^{d-1}} \mathcal{y}^{\text {an }}\left(\right.$ resp. $\left.Z_{i}^{\prime \prime}=X^{\text {an }} \times_{\mathbf{A}^{d-1}} Y_{i}\right)$. Thus, the situation is reduced to the case when $X$ (and therefore $Y$ ) is affinoid. In this case we use the results on the relative interior of a morphism from [Ber1, §2.5 and §3.1]. Since the canonical morphism $Y \rightarrow X$ (resp. $Y \stackrel{\sim}{\rightarrow} Y)$ is closed, it follows that the image of $Y$ in $Z$ is contained in $\operatorname{Int}(Z / X)$ $($ resp. Int $(Z / Y)$ ), the relative interior of $Z$ over $X($ resp. $Y)$. But $\operatorname{Int}(Z / X)=$ $\operatorname{Int}\left(Z / Z^{\prime}\right) \cap \operatorname{Int}\left(Z^{\prime} / X\right)\left(\operatorname{resp} . \operatorname{Int}(Z / Y)=\operatorname{Int}\left(Z / Z^{\prime \prime}\right) \cap \operatorname{Int}\left(Z^{\prime \prime} / Y\right)\right)$. It remains to use the facts that the morphism $Z^{\prime} \rightarrow X$ (resp. $Z^{\prime \prime} \rightarrow Y$ ) is closed, i.e., $\operatorname{Int}\left(Z^{\prime} / X\right)=Z^{\prime}\left(\right.$ resp. $\left.\operatorname{Int}\left(Z^{\prime \prime} / Y\right)=Z^{\prime \prime}\right)$, and that $\operatorname{Int}\left(Z / Z^{\prime}\right)$ (resp. $\left.\operatorname{Int}\left(Z / Z^{\prime \prime}\right)\right)$ coincides with the topological interior of $Z$ in $Z^{\prime}$ (resp. $Z^{\prime \prime}$ ).

Step 3. There is a strong deformation retraction of $X=Y \times D(0, r)$ to $Y \times\{0\}$. Consider the mapping $Y \times[0, r[\rightarrow X$ that takes a pair $(y, t)$ to the maximal point of the closed disc $E(0, t)_{\mathscr{H}(y)}$ (over the point $y$ ). From [Ber1, Lemma 6.1.1], it follows that this mapping is continuous and identifies $Y \times[0, r[$ with a closed subset of $X$. Furthermore, the canonical action of the multiplicative group $G_{\mathrm{m}}=\mathbf{A}^{1} \backslash\{0\}$ on $\mathbf{A}^{1}$ induces an action of the affinoid torus $G_{\mathrm{m}}^{1}=\left\{y \in \mathbf{A}^{1}|| T(y) \mid=1\right\}$ on $D(0, r)$. The latter induces an action of $G_{\mathrm{m}}^{1}$ on $X=Y \times D(0, r)$. For $0 \leq t<1$, let $g_{t}$ be the maximal point of the closed disc of radius $t$ with center at 1 (it is contained in $G_{\mathrm{m}}^{1}$ ), and let $g_{1}$ be the maximal point of $G_{\mathrm{m}}^{1}$. By [Ber1, §6.1], the continuous mapping $\Phi: X \times[0,1] \rightarrow X$ that takes $(y, t)$ to $g_{t} * y$ is a strong deformation retraction of $X$ to the closed subset $Y \times[0, r[$. But $Y \times\{0\}$ is evidently a strong deformation retraction of the latter space. Thus, the local contractibility of $X$ at $x$ is reduced to that of $Y$ at $x$.

Case (b). Since rational strictly $k$-affinoid neighborhoods of the point $x$ form a fundamental system of compact neighborhoods of $x$ in $X^{\text {an }}$, it suffices to show that, given a rational strictly $k$-affinoid neighborhood $W$ of $x$ in $X^{\text {an }}$, there exists a contractible open neighborhood of $x$ in $X$ contained in $W \cap X$.

Step 1 . The construction of the first step works in a more general setting and does not use the assumption (b). Since it will also be used in the next section, we formulate it as a lemma.

Lemma 9.4. Suppose we are given an integral affine scheme $\mathcal{X}=\operatorname{Spec}(A)$ of finite type over $k$, a compact strictly $k$-analytic domain $X \subset X^{\text {an }}$, a point $x \in X$, and a rational strictly $k$-affinoid neighborhood $W$ of $x$ in $X^{\text {an }}$. Then there is an open embedding of $\mathcal{X}$ in $\mathcal{y}_{\eta}$, where $\mathcal{y}_{\text {is }}$ an integral scheme proper finitely presented and flat over $k^{\circ}$, open subschemes $\mathcal{Z}$ and $\mathcal{W}$ of $\mathcal{Z}_{s}$, and a closed subscheme $\mathcal{V}$ of $\mathcal{Y}_{s}$ such that

(1) $X=\pi^{-1}(\mathcal{Z}), W=\pi^{-1}(\mathfrak{W})$ and $\pi(x) \in \mathcal{V}$;

(2) $\mathcal{V} \subset \mathcal{W}$;

(3) $\mathcal{V}$ and $\mathcal{Y}_{s} \backslash \mathcal{Z}$ are unions of irreducible components of $\mathcal{y}_{s}$.

Here $\pi$ denotes the reduction map $y_{\eta}^{\text {an }} \rightarrow y_{s}$. (Recall (see [Ber3, §5]) that since $y$ is proper over $k^{\circ}$ the $k$-analytic space $y_{\eta}^{\text {an }}$ coincides with the generic fiber $\widehat{y}_{\eta}$ of the formal completion $\widehat{y}$ of $y$ along the closed fibre.) 
Notice that the properties (1) and (2) imply that $\pi^{-1}(\mathcal{V}) \cap X$ is an open neighborhood of the point $x$ in $X$ contained in $W \cap X$.

Proof. Step A. Let $\alpha_{1}, \ldots, \alpha_{p}$ be generators of the $k$-algebra $A$. Multiplying them by an element of $k$, we may assume that $X \cup W \subset\{y \in$ $\left.\mathcal{X}^{\text {an }}|| \alpha_{i}(y) \mid \leq 1,1 \leq i \leq p\right\}$. Furthermore, let $\beta_{1}, \ldots, \beta_{q}$ be polynomials from $k^{\circ}\left[T_{1}, \ldots, T_{p}\right]$ which generate the kernel of the surjective homomorphism $k\left[T_{1}, \ldots, T_{p}\right] \rightarrow A: T_{i} \mapsto \alpha_{i}$. By [RaGr, Theorem 3.4.6], the $k^{\circ}$-saturation $\mathbf{a}$ of the ideal of $k^{\circ}\left[T_{1}, \ldots, T_{p}\right]$ generated by $\beta_{1}, \ldots, \beta_{q}$ is finitely generated, and we get an affine scheme $\mathcal{X}^{\prime}=\operatorname{Spec}\left(A^{\prime}\right)$, where $A^{\prime}=k^{\circ}\left[T_{1}, \ldots, T_{p}\right] / \mathbf{a}$, which is flat and finitely presented over $k^{\circ}$ and for which $\mathcal{X}_{\eta}^{\prime}=\mathcal{X}$ and $\widehat{X}_{\eta}^{\prime}=\left\{y \in \mathcal{X}^{\text {an }}|| \alpha_{i}(y) \mid \leq 1,1 \leq i \leq p\right\}$. Take an arbitrary open embedding of $\mathcal{X}^{\prime}$ in a scheme $\mathcal{Y}$ projective over $k^{\circ}$. We may assume that $X_{\eta}^{\prime}=\mathcal{X}$ is dense in $\mathcal{Y}$, and we may replace $\mathcal{Y}$ by its reduction.

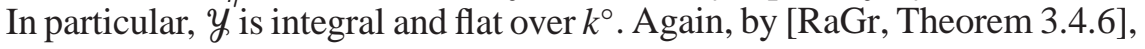
the scheme $y$ is finitely presented over $k^{\circ}$.

Our further constructions will modify $\mathcal{y}$ in the following way (see [RaGr, §5.1]). Suppose we are given a sheaf of ideals $g \subset \mathcal{O}_{y}$ of finite type. (From [RaGr, 3.4.6], it follows that $g$ is a coherent sheaf of ideals.) Furthermore, suppose that $\mathcal{g}$ contains a non-zero element of $k^{\circ \circ}$, and let $f: y^{\prime} \rightarrow y$ be the blow-up of $g$. The scheme $y^{\prime}$ is of finite type over $k^{\circ}$, and therefore if we divide $\mathcal{O}_{y^{\prime}}$ by the $k^{\circ}$-torsion, we get, by [RaGr, 3.4.6], a scheme $y^{\prime \prime}$ proper flat and finitely presented over $k^{\circ}$. We'll say that $y^{\prime \prime}$ is the blow-up of the ideal $g$ or, if $Y$ is the Zariski closed subset of $y_{s}$ which is the support of $\mathcal{O} y / g$, that $y^{\prime \prime}$ is the blow-up with center at $Y$. Note that the preimage of $Y$ in $y_{s}^{\prime \prime}$ is a union of irreducible components. Note also that $y_{\eta}^{\prime \prime} \stackrel{\sim}{\rightarrow} y_{\eta}$.

Step B. The strictly $k$-affinoid domain $W$ is rational. This implies that there are elements $f_{1}, \ldots, f_{n}, g \in A^{\prime}$ such that $g$ is invertible on $W$ and $W=\left\{y \in X^{\text {an }}|| f_{i}(y)|\leq| g(y) \mid, 1 \leq i \leq n\right\}$. By [EGAI, 6.9.7], there exists a coherent sheaf of ideals $\mathcal{g} \subset \mathcal{O}_{y}$ whose restriction to $X^{\prime}$ is generated by the elements $f_{1}, \ldots, f_{n}, g$. Let $\mathcal{y}^{\prime} \rightarrow \mathcal{Y}$ be the blow-up of the ideal $g$ (in the above sense). The " $g \neq 0$ " chart $\mathcal{W}$ of the preimage of $\mathcal{X}^{\prime}$ in $\mathcal{Y}^{\prime}$ is an open affine subscheme of $\mathcal{y}^{\prime}$ and one has $\pi^{-1}\left(\mathcal{W}_{s}\right)=W$.

We claim that the Zariski closure of the point $\pi(x)$ in $y_{s}^{\prime}$ is contained in $\mathcal{W}_{s}$. Indeed, for this it suffices to verify that, given an open affine subscheme $U \subset \mathcal{Y}^{\prime}$ that contains the point $x$, the Zariski closure of $\pi(x)$ in $U_{s}$ is contained in $U_{s} \cap \mathcal{W}_{s}$. If $\mathcal{U}=\operatorname{Spec}(B)$ and $U \cap \mathcal{W}=\operatorname{Spec}\left(B^{\prime}\right)$, the latter means that $\widetilde{B} / \wp \stackrel{\sim}{\rightarrow} \widetilde{B^{\prime}} / \wp \widetilde{B}^{\prime}$, where $\wp$ is the prime ideal of the point $\pi(x)$ in $\widetilde{B}=B / k^{\circ \circ} B_{2}$ and $\widetilde{B}^{\prime}=B^{\prime} / k^{\circ \circ} B^{\prime}$. Since $\operatorname{Spec}\left(\widetilde{B}^{\prime} \downarrow{ }^{\prime} \widetilde{B}^{\prime}\right)$ is an open subscheme of $\operatorname{Spec}(\widetilde{B} / \wp)$, it suffices to check that $\widetilde{B}^{\prime} / \wp \widetilde{B}^{\prime}$ is integral over $\widetilde{B} / \wp$. For this we use the assumption that the point $x$ is contained in the topological interior of $W$ in $y_{\eta}^{\prime a n}$. It implies that $x$ is contained in the topological interior of $U \cap W$ in $U$, where $U=\widehat{U}_{\eta}=\pi^{-1}\left(u_{s}\right)$. One has $U=\mathcal{M}(\mathscr{B})$ and $U \cap W=\mathcal{M}\left(\mathscr{B}^{\prime}\right)$ with $\mathscr{B}=\widehat{B} \otimes_{k^{\circ}} k$ and $\mathscr{B}^{\prime}=\widehat{B}^{\prime} \otimes_{k^{\circ}} k$, where $\widehat{B}$ and $\widehat{B}^{\prime}$ are the $a$-adic completions of $B$ and $B^{\prime}$, respectively for 
any $a \in k^{\circ \circ} \backslash\{0\}$. From [Ber1, Proposition 2.5.2], it follows that $\widetilde{\mathscr{B}}^{\prime} / \wp^{\prime} \widetilde{\mathcal{B}}^{\prime}$ is integral over $\widetilde{B} / \wp^{\prime}$, where $\wp^{\prime}$ is the prime ideal of the point $\pi^{\prime}(x)$ and $\pi^{\prime}$ is the reduction map $U \rightarrow \widetilde{U}=\operatorname{Spec}(\widetilde{\mathscr{B}})$. Since $\widetilde{\mathcal{B}}$ and $\widetilde{\mathcal{B}}^{\prime}$ are integral over $\widetilde{B}$ and $\widetilde{B}^{\prime}$, respectively (see [Ber $\left.3, \S 1\right]$ ), and $\wp$ is the preimage of $\wp^{\prime}$ with respect to the homomorphism $\widetilde{B} \rightarrow \widetilde{B}$, the required fact follows.

We replace $y$ by $y^{\prime}$. In what follows we use only the closed fibre of the subscheme $\mathcal{W}$, and therefore we denote it by $\mathcal{W}$. We remark that, given a blow-up $y^{\prime} \rightarrow \mathcal{Y}$, the preimage $\mathcal{W}^{\prime}$ of $\mathcal{W}$ in $\mathcal{Y}_{s}^{\prime}$ possesses the same property: the Zariski closure of the point $\pi(x)$ in $y_{s}^{\prime}$ is contained in $\mathcal{W}^{\prime}$.

Step C. By Gerritzen-Grauert Theorem [BGR, 7.3.5/3], $X$ is a union of rational strictly affinoid domains $X_{1}, \ldots, X_{p}$. For each $X_{i}$ we apply the construction from the beginning of Step B, and we get a blow-up $y_{i} \rightarrow y$ and an open subscheme $\mathcal{Z}_{i}$ of the closed fibre of $\mathcal{Y}_{i}$ such that $\pi^{-1}\left(\mathcal{Z}_{i}\right)=X_{i}$. We now can find a blow-up $y^{\prime} \rightarrow y$ that goes through all of the above blowups. Let $\mathcal{Z}$ be the union of the preimages of $\mathcal{Z}_{i}$ in $\mathcal{Y}_{s}^{\prime}$. Then $\pi^{-1}(\mathcal{Z})=X$. Finally, we make two additional blow-ups with centers in $\mathcal{Y}_{s}^{\prime} \backslash \mathcal{Z}$ and in the Zariski closure of $\pi(x)$ in $\mathcal{Y}_{s}^{\prime}$. We replace $\mathcal{Y}$ by $\mathcal{Y}^{\prime}, \mathcal{Z}$ and $\mathcal{W}$ by their preimages in $\mathcal{Y}_{s}^{\prime}$, and denote by $\mathcal{V}$ the preimage of the Zariski closure of $\pi(x)$. By Step B, $\mathcal{V} \subset \mathcal{W}$ and, by the last construction, $\mathcal{V}$ and $\mathcal{Y}_{s} \backslash \mathcal{Z}$ are unions of irreducible components.

Step 2. One may assume that the scheme $\mathcal{X}$ is geometrically irreducible. Indeed, the algebraic separable closure $k^{\prime}$ of $k$ in $A$ is finite over $k$, and the scheme $\mathcal{X}$, considered as a smooth scheme over $k^{\prime}$, is geometrically irreducible [EGAIV, 4.5.9]. On the other hand, $X$ can be considered as a strictly $k^{\prime}$-analytic space, and so we can replace $k$ by $k^{\prime}$ and reduce the situation to the case when $\mathcal{X}$ is geometrically irreducible.

Step 3. Applying Lemma 9.4 to $\mathcal{X}, X, W$ and $x$, and we get $\mathcal{Z}, \mathcal{Z}, \mathcal{W}$ and $\mathcal{V}$ satisfying the properties (1)-(3). Since $\mathcal{X}$ is geometrically irreducible, $y_{\eta}$ is also geometrically irreducible. By Lemma 9.2, there exist a finite normal extension $k^{\prime}$ of $k$, a poly-stable fibration $\underline{y^{\prime}}$ of length $l$ over $k^{\prime \circ}$ such that $y_{l}^{\prime}$ is proper over $k^{\prime \circ}$ and has a smooth geometrically irreducible generic fibre, an action of a finite group $G$ on $\underline{y}^{\prime}$ over $k^{\circ}$, and a dominant $G$ equivariant morphism $\varphi: y_{l}^{\prime} \rightarrow y_{\text {that }}$ thaces a proper generically finite morphism $y_{l, \eta}^{\prime} \rightarrow y_{\eta}$ and such that the field $R\left(y_{l, \eta}^{\prime}\right)^{G}$ is purely inseparable over $R(y)$.

Step 4. Let $\mathcal{Z}^{\prime}, \mathcal{W}^{\prime}$ and $\mathcal{V}^{\prime}$ be the preimages of $\mathcal{Z}, \mathcal{W}$ and $\mathcal{V}$ in $\mathcal{Y}_{l, s}^{\prime}$, respectively. Then $\mathcal{V}^{\prime}$ and $\mathcal{Y}_{l, s}^{\prime} \backslash \mathcal{Z}^{\prime}$ are unions of irreducible components of $\mathcal{Y}_{l, s}^{\prime}$ and $\mathcal{V}^{\prime} \subset \mathcal{W}^{\prime}$. For $X^{\prime}=\pi^{-1}\left(\mathcal{Z}^{\prime}\right)$ and $W^{\prime}=\pi^{-1}\left(\mathcal{W}^{\prime}\right)$, one has $X^{\prime}=\varphi_{\eta}^{-1}(X)$ and $W^{\prime}=\varphi_{\eta}^{-1}(W)$. Moreover, $\pi^{-1}\left(\mathcal{V}^{\prime}\right) \cap X^{\prime}$ is an open subset of $X^{\prime}$ contained in $W^{\prime} \cap X^{\prime}$. By the construction, we can find a nonempty open affine subscheme $\mathcal{U} \subset \mathcal{X}$ such that the morphism $\mathcal{U}^{\prime}:=\varphi_{\eta}^{-1}(\mathcal{U}) \rightarrow \mathcal{U}$ is finite and the finite morphism $G \backslash \mathcal{U}^{\prime} \rightarrow U$ is radicial. By the assumption (b), the point $x$ is contained in $\mathcal{U}^{\text {an }}$. Then the set $U:=\pi^{-1}(\mathcal{V}) \cap X \cap \mathcal{U}^{\text {an }}$ is an open neighborhood 
of $x$ in $X$ contained in $W \cap X$. The set $U^{\prime}:=\pi^{-1}\left(\mathcal{V}^{\prime}\right) \cap X^{\prime} \cap U^{\prime \text { an }}$ is open in $X^{\prime}$ and dense Zariski open in $\pi^{-1}\left(\mathcal{V}^{\prime}\right) \cap X^{\prime}=\pi^{-1}\left(\mathcal{V}^{\prime} \cap \mathcal{Z}^{\prime}\right)$, and the radicial morphism $G \backslash \mathcal{U}^{\prime} \rightarrow \mathcal{U}$ induces a homeomorphism $G \backslash U^{\prime} \stackrel{\sim}{\rightarrow} U$. Since $\mathcal{V}^{\prime}$ and $\mathcal{Y}_{l, s}^{\prime} \backslash \mathcal{Z}^{\prime}$ are unions of irreducible components of $\mathcal{y}_{l, s}^{\prime}$, it follows that $\mathcal{V}^{\prime} \cap \mathcal{Z}^{\prime}$ is a strata subset of $\mathcal{Y}_{l, s}^{\prime}$. By Corollary 8.5(i), there is a strong deformation retraction of $U$ to a closed subset homeomorphic to $G \backslash\left|\mathbf{C}\left(\mathcal{V}^{\prime} \cap \mathcal{Z}^{\prime}\right)\right|$ and, by Corollary 3.11(iii), the latter space is locally contractible. The theorem follows.

Corollary 9.5. Any separated $k$-analytic space $X$ locally embeddable in a smooth $k$-analytic space is locally simply connected. In particular, if $X$ is connected, it has a universal covering which is a simply connected strictly $k$-analytic space.

We remark that since smooth $k$-analytic spaces are locally separated, a $k$-analytic space locally embeddable in a smooth $k$-analytic space is separated if and only if it is Hausdorff (see [Ber2, §1.4]). Corollary 9.5 implies that the topological fundamental group $\pi_{1}^{\text {top }}(X)$ of such a space $X$, defined in [deJ1] as a topological group classifying the topological coverings of $X$, coincides with the usual fundamental group of $X$, defined through paths on $X$. In particular, $\pi_{1}^{\text {top }}(X)$ is a discrete group.

Corollary 9.6. Any separated k-analytic space X locally embeddable in a smooth $k$-analytic space is homologically locally connected. In particular, if $X$ is paracompact, then for any abelian group $M$ the canonical maps $H^{q}(|X|, M) \rightarrow H_{\text {sing }}^{q}(|X|, M)$ are isomorphisms.

Remark 9.7. Any strictly $k$-affinoid space smooth in the sense of rigid geometry is locally embeddable in a smooth space. Indeed, if $X=\mathcal{M}(\mathcal{A})$ be such a space, then the sheaf of one-differentials $\Omega_{X}$ is locally free of rank $n=\operatorname{dim}(X)$. This implies that, given a point $x \in X$, the restriction of $\Omega_{X}$ to a Zariski open neighborhood of $x$ is free, i.e., shrinking $X$, we may assume that $\Omega_{X}$ is generated by $d f_{1}, \ldots, d f_{n}$ for some $f_{i} \in \mathcal{A}^{\circ}, 1 \leq i \leq n$. Consider the morphism $f=\left(f_{1}, \ldots, f_{n}\right): X \rightarrow E^{n} \subset \mathbf{A}^{n}$. By the results of R. Elkik [Elk, Ch. III, Theorem 7, Lemma 6 and Remark 2], one can approximate the morphism $f$ by a morphism which comes from a morphism of smooth affine schemes $\mathcal{X} \rightarrow A^{n}$ so that $X$ is isomorphic to the preimage of $E^{n}$ in $\mathcal{X}^{\text {an }}$.

\section{Finiteness and stability of cohomology of certain analytic spaces}

In this section the valuation on the ground field $k$ is not assumed to be non-trivial.

Theorem 10.1. Let $X$ be a k-analytic space isomorphic to $W \backslash \mathcal{V}^{\text {an }}$, where $W$ is a compact analytic domain in the analytification $X^{\text {an }}$ of a separated scheme $\mathcal{X}$ of finite type over $k$ and $\mathcal{V}$ is a Zariski closed subset of $\mathcal{X}$. Then 
(i) the groups $H^{q}(|X|, \mathbf{Z})$ are finitely generated;

(ii) if $W$ is strictly $k$-analytic, then there exists a finite separable extension $k^{\prime}$ of $k$ such that, for any non-Archimedean field $K$ over $k^{\prime}$ and any paracompact locally closed subset $Y$ of a Hausdorff $K$-analytic space, one has

$$
R \Gamma\left(\left|X \widehat{\otimes} k^{\prime}\right|, \mathbf{Z}\right) \stackrel{\mathbf{L}}{\otimes} R \Gamma(|Y|, \mathbf{Z}) \stackrel{\sim}{\rightarrow} R \Gamma(|X \times Y|, \mathbf{Z})
$$

and, in particular, $H^{q}\left(\left|X \widehat{\otimes} k^{\prime}\right|, \mathbf{Z}\right) \stackrel{\sim}{\rightarrow} H^{q}(|X \widehat{\otimes} K|, \mathbf{Z})$.

Proof. We use a construction of P. Deligne from [Del] (which is also mentioned at the beginning of J. de Jong's paper [deJ2]). For this we remark that if there exists a proper hypercovering $X . \rightarrow X$ such that the theorem is true for all $X_{n}$ 's, then it is also true for $X$ (see [SGA4, Exp. V bis]). Consider first the case when the compact analytic domain $W$ is strictly $k$-analytic.

First, we may assume that $\mathcal{X}$ is proper. By the above remark and Chow's Lemma, we may assume that $\mathcal{X}$ is projective. Furthermore, by the above remark, we can replace $\mathcal{X}$ by its normalization and, therefore, we may assume that $\mathcal{X}$ is irreducible and integral. Using the reasoning from Step A from the proof of Lemma 9.4, we may assume that $\mathcal{X}$ is the generic fibre

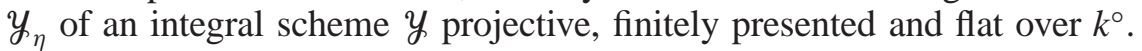
Finally, by Step C from the proof of Lemma 9.4, we may assume that $W=\pi^{-1}(\mathcal{Z})$, where $\mathcal{Z}$ is an open subscheme of the closed fibre $\mathcal{Y}_{s}$ such that $\mathcal{y}_{s} \backslash \mathcal{Z}$ is a union of irreducible components of $\mathcal{y}_{s}$.

By the construction from [Del, 6.3.5], and Lemma 9.2, there exist

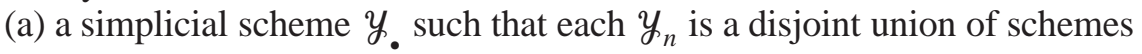
of the form $\mathcal{X}_{l}$, where $\underline{\mathcal{X}}=\left(\mathcal{X}_{l} \stackrel{f_{l-1}}{\rightarrow} \ldots \stackrel{f_{1}}{\rightarrow} \mathcal{X}_{1} \stackrel{f_{0}}{\rightarrow} \mathcal{X}_{0}=\operatorname{Spec}\left(k^{\prime}\right)\right)$ is a poly-stable fibration over a finite extension $k^{\prime}$ of $k$ and all $f_{i}$ 's are projective and have smooth generic fibres, and (b) an augmentation $g: y . \rightarrow y$ which gives rise to a proper hypercovering $g_{\eta}: y_{. \eta \eta} \rightarrow y_{\eta}$. The latter gives rise to a proper hypercovering $X . \rightarrow X$, where $X_{n}$ is the preimage of $X$ in $\mathcal{Y}_{n, \eta}^{\text {an }}$. One has $X_{n}=\pi^{-1}\left(\mathcal{Z}_{n}\right) \backslash \mathcal{V}_{n}^{\text {an }}$, where $\mathcal{Z}_{n}$ is the preimage of $\mathcal{Z}$ in $\mathcal{Y}_{n, s}$ and $\mathcal{V}_{n}$ is the preimage of $\mathcal{V}$ in $\mathcal{Y}_{n, \eta}$. Since $\mathcal{Y}_{s} \backslash \mathcal{Z}$ is a union of irreducible components of $\mathcal{y}_{s}$, it follows that $\mathcal{y}_{n, s} \backslash \mathcal{Z}_{n}$ is a union of irreducible components of $\mathcal{Y}_{n, s}$ and, therefore, $\mathcal{Z}_{n}$ is a strata subset of $\mathcal{Y}_{n, s}$. The statement (i) for $X_{n}$ now follows from Corollary 8.5. Corollary 8.7 implies the existence of $k^{\prime}$ such that for any $Y$ from (ii) the canonical maps $\left|X_{n} \times Y\right| \rightarrow\left|X_{n}\right| \times|Y|$ are homotopy equivalences. Since $X_{n}$ and $Y$ are locally compact and paracompact and the above map is compact, the spaces $X_{n} \times Y$ and $\left|X_{n}\right| \times|Y|$ are also locally compact and paracompact. It remains to apply the facts that for such a space the cohomology groups coincides with the Alexander cohomology groups and that the latter satisfy the homotopy axiom (see [Spa, Ch. 6]).

Consider now the general case when $W$ is not necessarily strictly $k$-analytic. We can find positive numbers $r_{1}, \ldots, r_{n}$, whose images in the quotient group $\mathbf{R}_{+}^{*} / \sqrt{\left|k^{*}\right|}$ are linearly independent over $\mathbf{Q}$, such that $W \widehat{\otimes} K$ is strictly $K$-analytic, where $K=K_{r_{1}, \ldots, r_{n}}$ (see [Ber1, §2.1]). The field $K$ 
is isomorphic to the $k$-affinoid algebra of analytic functions on the direct product $A\left(r_{1}\right) \times \cdots \times A\left(r_{n}\right)$, where $A(r)=\left\{x \in \mathbf{A}^{1}|| T(x) \mid=r\right\}$. Thus, to prove the statement (i) it suffices to check that for any Hausdorff $k$-analytic space $X$ and any number $r>0$ the canonical map $|X \times A(r)| \rightarrow|X|$ is a homotopy equivalence. For this we note that the canonical action of the multiplicative group $G_{\mathrm{m}}$ on $\mathbf{A}^{1}$ induces an action of the affinoid torus $G_{\mathrm{m}}^{1}$ on $A(r)$. As it was explained in Step 3 (Case (a)) from the proof of Theorem 9.1, the latter action gives rise to a strong deformation retraction of $X \times A(r)$ to a closed subset homeomorphic to $X$, and the statement (i) follows.

Corollary 10.2. In the situation of Theorem 10.1, the following is true

(i) the groups $H_{c}^{q}(|X|, \mathbf{Z})$ are finitely generated;

(ii) if $W$ is strictly $k$-analytic, then there exists a finite separable extension $k^{\prime}$ of $k$ such that, for any non-Archimedean field $K$ over $k^{\prime}$ and any locally closed subset $Y$ of a Hausdorff $K$-analytic space, one has

$$
R \Gamma_{c}\left(\left|X \widehat{\otimes} k^{\prime}\right|, \mathbf{Z}\right) \stackrel{\mathbf{L}}{\otimes} R \Gamma_{c}(|Y|, \mathbf{Z}) \stackrel{\sim}{\rightarrow} R \Gamma_{c}(|X \times Y|, \mathbf{Z})
$$

and, in particular, $H_{c}^{q}\left(\left|X \widehat{\otimes} k^{\prime}\right|, \mathbf{Z}\right) \stackrel{\sim}{\rightarrow} H_{c}^{q}(|X \widehat{\otimes} K|, \mathbf{Z})$.

Notice that the statements (i) and (ii) of Theorem 10.1 and Corollary 10.2 are true for $X=\mathcal{X}^{\text {an }}$, where $\mathcal{X}$ is a separated scheme of finite type over $k$. Indeed, there is an open embedding of $\mathcal{X}$ in a proper scheme $\mathcal{Y}$. The analytic space $\mathcal{Y}^{\text {an }}$ is compact and strictly $k$-analytic, and one has $X^{\text {an }}=y^{\text {an }} \backslash \mathcal{V}^{\text {an }}$, where $\mathcal{V}$ is the complement of $\mathcal{X}$ in $\mathcal{y}$.

\section{References}

[Ber1] Berkovich, V. G.: Spectral theory and analytic geometry over non-Archimedean fields, Mathematical Surveys and Monographs, vol. 33, American Mathematical Society, Providence, R.I., 1990

[Ber2] Berkovich, V. G.: Étale cohomology for non-Archimedean analytic spaces, Publ. Math. IHES 78, 5-161 (1993)

[Ber3] Berkovich, V. G.: Vanishing cycles for formal schemes, Invent. Math. 115, 539571 (1994)

[Ber4] Berkovich, V. G.: On the comparison theorem for étale cohomology of nonArchimedean analytic spaces, Israel J. Math. 92, 45-60 (1995)

[Ber5] Berkovich, V. G.: The automorphism group of the Drinfeld half-plane, C. R. Acad. Sci. Paris Sér. I Math. 321, 1127-1132 (1995)

[Ber6] Berkovich, V. G.: Vanishing cycles for formal schemes. II, Invent. Math. 125, 367-390 (1996)

[Ber7] Berkovich, V. G.: Vanishing cycles for non-Archimedean analytic spaces, J. Amer. Math. Soc. 9, 1187-1209 (1996)

[BGR] Bosch, S., Güntzer, U., Remmert, R.: Non-Archimedean analysis. A systematic approach to rigid analytic geometry, Grundlehren der Mathematischen Wissenschaften, Bd. 261, Springer, Berlin-Heidelberg-New York 1984

[BrTi] Bruhat, F., Tits, J.: Groupes réductifs sur un corps local. I. Donnée radicielles valuée, Publ. Math. IHES 41, 5-251 (1972) 
[Che] Cherry, W.: Non-Archimedean analytic curves in abelian varieties, Math. Ann. 300, 393-404 (1994)

[Del] Deligne, P.: Théorie de Hodge, III, Publ. Math. IHES 44, 5-78 (1974)

[Dr1] Drinfeld, V. G.: Elliptic modules, Math. USSR Sbornik, 23 561-592 (1974)

[Dr2] Drinfeld, V. G.: Coverings of $p$-adic symmetric domains, Funct. Anal. Appl. 10, 107-115 (1976)

[EGAI] Grothendieck, A., Dieudonné, J.: Eléments de Géométrie Algébrique. I. Le langage des schémas, Springer, Berlin-Heidelberg-New York 1971

[EGAIV] Grothendieck, A., Dieudonné, J.: Eléments de Géométrie Algébrique. IV. Étude local des schémas et des morphismes de schémas, Publ. Math. IHES 20 (1964), 25 (1965), 28 (1966), 32 (1967)

[Elk] Elkik, R.: Solutions d'équations à coefficients dans un anneau hensélien, Ann. Scient. Éc. Norm. Sup. 6, 553-604 (1973)

[En] Engelking, R.: General topology, Warszawa 1977

[GaZi] Gabriel, P., Zisman, M.: Calculus of fractions and homotopy theory, Ergebnisse der Mathematik und ihrer Grenzgebiete, Bd. 35, Springer, Berlin-HeidelbergNew York 1967

[Hall] Hall, M.: The theory of groups, The Macmillan Company, New-York 1959

[deJ1] de Jong, A. J.: Étale fundamental group of non-Archimedean analytic spaces, Compos. Math. 97, 89-118 (1995)

[deJ2] de Jong, A. J.: Smoothness, semi-stability and alterations, Publ. Math. IHES 83, 51-93 (1996)

[deJ3] de Jong, A. J.: Families of curves and alterations, Ann. Inst. Fourier (Grenoble), 47, 599-621 (1997)

[Kato] Kato, K.: Logarithmic structures of Fontaine-Illusie, Algebraic analysis, geometry, and number theory, Johns Hopkins University Press, Baltimore, 191-224, 1989

[Lang] Lang, S.: Algebra, Addison-Wesley, Reading, Mass. 1965

[Mac] MacLane, S.: Categories for the Working Mathematician, Graduated Texts in Mathematics, Vol. 5, Springer, Berlin-Heidelberg-New York 1971

[Mus] Mustafin, G. A.: Non-Archimedean uniformization, Math. USSR Sbornik 34, 107-115 (1978)

[Ra] Raynaud, M.: Anneaux Locaux Henséliens, Lecture Notes in Math. 169, Springer, Berlin-Heidelberg-New York 1970

[RaGr] Raynaud, M., Gruson, L.: Critères de platitudes et de projectivité, Invent. Math. 13, 1-89 (1971)

[Spa] Spanier, E. H.: Algebraic topology, McGraw-Hill Book Company, New YorkSan Francisco-Toronto-London-Sydney 1966

[SGA1] Grothendieck, A.: Revêtements Etales et Groupe Fondamental, Lect. Notes Math. 224, Springer, Berlin-Heidelberg-New York 1971

[SGA4] Artin, M., Grothendieck, A., Verdier, J.-L.: Théorie des Topos et Cohomologie Étale des Schémas, Lect. Notes Math. 269, 270, 305, Springer, BerlinHeidelberg-New York 1972-1973

[Tate] Tate, J.: Rigid analytic spaces, Invent. Math. 12, 257-289 (1971)

[Tsu] Tsuji, T.: P-adic étale cohomology and crystalline cohomology in the semi-stable reduction case, Invent. Math. (to appear)

[Var] Varshavsky, Y.: P-adic uniformization of unitary Shimura varieties. II, J. Differ. Geom. 49, 75-113 (1998) 